\title{
Sound Generation by Turbulent Premixed Flames
}

\author{
Ali Haghiri \\ Department of Mechanical Engineering \\ The University of Melbourne
}

Submitted in total fulfilment of the requirements of the degree of

Doctor of Philosophy

August 2017 
Copyright (C) 2017 Ali Haghiri

All rights reserved. No part of the publication may be reproduced in any form by print, photoprint, microfilm or any other means without written permission from the author. 
I would like to dedicate this thesis to my wife and parents who contributed no less than myself towards the completion of my doctoral studies. 


\section{Declaration}

This is to certify that:

1. the thesis comprises only my original work towards the PhD except where indicated,

2. due acknowledgement has been made in the text to all other material used,

3. the thesis is less that 100,000 words exclusive of tables, maps, bibliographies and appendices.

Signed,

Ali Haghiri

August 2017 


\section{Acknowledgements}

First and foremost, I would like to gratefully thank my supervisors, Dr. Mohsen Talei and Prof. Michael Brear, for their detailed guidance and enthusiastic support throughout my Ph.D. studies. They were always willing to listen patiently and to discuss about any aspect of the research. Their motivation and immense knowledge are truly acknowledged. Without their support, the work in this thesis would not have been possible. I would also like to thank Dr. Yi Yang and Dr. Daniel Chung for accepting to be on my advisory committee and providing useful comments. Many thanks also to Dr. Robert Gordon for participating in our weekly combustion noise group meeting for his good advice and useful comments.

In addition, I would like to take this opportunity to express my thanks to Prof. Evatt Hawkes for providing me with HPC resources. I also really appreciate the time that I spent working with him and his team as a visiting researcher in UNSW for three months in early 2013. In particular, Dr. Shahram Karami deserves a special thank for teaching me about using the DNS solver S3D-SC and code compilation. During this short period, I could learn a lot about the unique aspects of high performance computing and DNS of combustion.

I would also like to express my gratitude to all my friends in the Thermodynamics Group for their friendship and help whenever needed. This includes previous and present lab-mates: Tien, Kai, Peter, Pedro, Tim, Farzad, Matthew B., Sam, Joshua, Matthew J., Abbas, Chen, Song, Hao, Daniel, Victor, Jacob, Avishai, Davy, Mostafa, Aditiya and many more for making this Ph.D. more than an academic 
experience. Monica Pater is also gratefully acknowledged for her help in kindly doing administrative duties.

I would like to deeply thank my wife, Marzieh, for her endless love, support and patience throughout these years, while she has been busy with her own Ph.D. at the Faculty of Medicine. She has made countless sacrifices in order for me to accomplish this chapter of my life. I cannot also give sufficient appreciation to my parents, Mohammad and Tayebeh. I am profoundly grateful for their great encouragement and unconditional support. Nothing that I do, can show my gratitude for you! Encouragement from my brothers, Amin and in particular Hamed, to me for pursuing my $\mathrm{PhD}$ studies abroad is truly acknowledged. Thanks also to my parents in law, Mohammad Ali and Fatemeh, for their support.

Finally, I would like to acknowledge that this study was supported by the Advanced Centre for Automotive Research and Testing (ACART, www.acart.com.au), the Australian Research Council (ARC) and the Australian Government's National Computational Merit Allocation Scheme. The computational facilities supporting this project included the Australian National Facility (NCI), the partner share of the NCI facility provided by Intersect Australia Pty Ltd. and the Pawsey Supercomputing Centre. 


\begin{abstract}
In addition to being a significant form of noise pollution, combustion noise plays a key role in the instability of many combustion systems. The noise generated by turbulent premixed flames deserves special attention since they are common in contemporary, low-emission gas turbines. Hence, a detailed understanding of the mechanism of sound generation by premixed flames is needed for designing quieter and safer combustion systems.

This thesis presents a study of sound generation by turbulent, premixed flames using both direct numerical simulation (DNS) and theory. DNS of two round jet methane/air flames with equivalence ratios of 0.7 and 1.0 are carried out. Single step chemistry is employed to reduce the computational cost, and care is taken to resolve both the near and far fields and to avoid noise reflections at the outflow boundaries.

Several significant features of these two flames are noted. These include the monopolar nature of the sound from both flames, the stoichiometric flame being significantly louder than the lean flame, and the observed frequency of peak acoustic spectral amplitude being consistent with prior experimental studies. Further, the importance of different types of so-called "flame annihilation" events as monopolar acoustic sources is demonstrated. A simple model describing the contribution of annihilation events to the far-field sound is then proposed. This model considers the far-field sound generated by a turbulent premixed flame as the superposition of the sound radiated by individual, spherically symmetric annihilation events that occurred throughout
\end{abstract}


the flame. The results show a significant contribution by these events to the overall produced sound.

A statistical analysis of the data pertaining to annihilation events is then presented. The results show that annihilation events generally feature high negative values of curvature. A strong negative correlation between the flame displacement speed and curvature is also observed. Examining different terms of the flame displacement speed during flame annihilation reveals that the reaction term has the highest contribution among the others, followed by the curvature term and lastly the normal diffusion term. The Markstein number $(M a)$ is also calculated for both the entire flame and annihilation events. Smaller values of $M a$ are obtained for the annihilation events. Finally, a new linear model for response of turbulent premixed flames to stretch during flame annihilation is proposed. 


\section{Contents}

Contents viii

List of Figures

List of Tables $\quad$ Xviii

1 Introduction 1

1.1 Motivation . . . . . . . . . . . . . . . . 1

1.2 Research Aims . . . . . . . . . . . . . 2

2 Literature Review 4

2.1 Introduction . . . . . . . . . . . . . . . . . . 4

2.2 Fundamentals of Combustion Noise . . . . . . . . . . 5

2.3 Lighthill's Acoustic Analogy . . . . . . . . . . . . . 7

2.3.1 Combusting Forms of Lighthill's Acoustic Analogy . . . . . 9

2.4 Turbulent Premixed Flames . . . . . . . . . . . . . . . . 11

2.4.1 Regime Diagram . . . . . . . . . . . . . . 11

2.4.2 Implications of Regime Diagram for Combustion Noise . . 13

2.5 Experimental Studies of Sound Generation by Premixed Flames . 14

2.6 Numerical Studies of Sound Generation by Premixed Flames . . . 17

2.6.1 LES Studies . . . . . . . . . . . . . . . . . . . . 18

2.6.2 DNS Studies . . . . . . . . . . . . . . . . . . . . 19

2.7 Summary . . . . . . . . . . . . . . . . . . . . . . . . . . . . . . . 24

3 Numerical Methods and DNS Setup

3.1 Introduction . . . . . . . . . . . . . . . . . . . 26 
3.2 Governing Equations $\ldots \ldots \ldots \ldots$

3.3 Boundary Conditions . . . . . . . . . . . . . . . . . 30

3.4 Numerical Solver . . . . . . . . . . . . . . . . . . . . . . 34

3.5 Flow Configuration and Numerical Setup . . . . . . . . . . 35

3.5.1 Computational Domain and Initial Conditions . . . . . 3. 35

3.5.2 Flame Parameters . . . . . . . . . . . . . . . . 35

3.5.3 Turbulence Feed Setup . . . . . . . . . . . . . . . 39

3.5.4 Grid Resolution . . . . . . . . . . . . . . . . . . . 39

3.5.5 Boundary Conditions and Sponge Layer Setup . . . . . . 43

3.5.6 DNS Run and Computational Cost . . . . . . . . . 4 46

3.6 Summary . . . . . . . . . . . . . . . . . . . . 48

4 DNS of Sound Generation by Turbulent Premixed Flames 49

4.1 Introduction . . . . . . . . . . . . . . . . . . . . . . . . 49 49

4.2 General Structure . . . . . . . . . . . . . . . . . . . . 49

4.3 Sound Generation . . . . . . . . . . . . . . . 56 5

4.4 Spectral Analysis . . . . . . . . . . . . . . 57

4.5 Conclusions . . . . . . . . . . . . . . . . . 65

5 Sound Generation by Annihilation Events in Turbulent Premixed Flames 66

5.1 Introduction . . . . . . . . . . . . . . . . 66

5.2 Identification of Flame Annihilation . . . . . . . . . . 66

5.3 A Simple Model of Sound Generation by Flame Annihilation . . . 70

5.3.1 Laminar Flame Annihilation . . . . . . . . . . . 70

5.3 .2 Theory . . . . . . . . . . . . . . . . 70

5.3.3 Model Development for Turbulent Premixed Flames . . . . 72

5.3.3.1 Model Description . . . . . . . . . . 72

5.3.3.2 Algorithm . . . . . . . . . . . . . . 74

5.4 Comparison of the Simple Model with DNS . . . . . . . 75

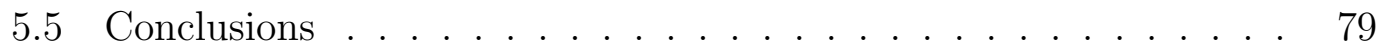

6 Annihilation Event Statistics in a Turbulent Premixed Flame 81

6.1 Introduction . . . . . . . . . . . . . . . 81 
6.2 Theoretical framework . . . . . . . . . . . . . . . 82

6.3 Flame Displacement Speed and Curvature . . . . . . . . . . . 84

6.4 Flame Stretch and Markstein Number . . . . . . . . . . . . . . 93

6.5 Contributions of Different Terms of Flame Displacement Speed to Noise Generation by Turbulent Premixed Flames . . . . . . . . . 99

6.6 A Revised Simple Model of Noise Generation by Turbulent Premixed Flames . . . . . . . . . . . . . . . . . . . . 100

6.7 Conclusions . . . . . . . . . . . . . . . . 102

7 Conclusions and Recommendations 103

7.1 Recommendations for Future Research . . . . . . . . . . . . . . 106

Bibliography 108

Appendix A: Calculations and Derivations

121 


\section{List of Figures}

1.1 (a) Thermoacoustic feedback cycle O'Connor et al. 2015; (b) Typical time history of pressure signal associated with a combustion instability Poinsot \& Veynante, 2005. . . . . . . . . 2 2

2.1 Schematic of sound radiation by a low Mach number jet (Dowling

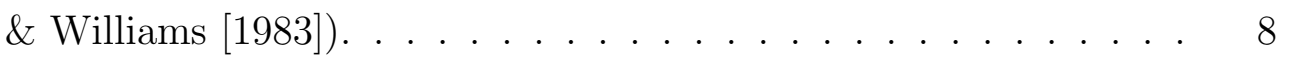

2.2 The regime diagram for premixed turbulent combustion Peters, 2000]. . . . . . . . . . . . . . . . . 13

2.3 (a) View of the "M" stabilised flame: steady flame (in the middle) and unstable flame regime with an oscillation frequency at $f=138$ $\mathrm{Hz}$ (a-h). (b) schematic representation of the feedback mechanism leading to self-sustained oscillations [Schuller et al. 2003]. . . . . 15

2.4 Typical spectral dependence of acetylene fuelled combustion noise (left), Strouhal number dependence of frequency of peak emission (right) Rajaram \& Lieuwen, 2009.

2.5 Non-dimensional pressure $\left(p-p_{\text {ref }}\right) / \rho_{\text {ref }} c_{\text {ref }}^{2}$ for flame annihilation from DNS (i) at the symmetry axis and (ii) in the far-field for different flame thicknesses, $\delta / L_{\text {ref }}=0.1$ (solid line), $\delta / L_{\text {ref }}=0.2$ (dashed), $\delta / L_{r e f}=0.4$ (dash-dotted). Talei et al., 2011] . . . . 20

2.6 Non-dimensional pressure $\left(p-p_{\text {ref }}\right) / \rho_{\text {ref }} c_{\text {ref }}^{2}$ for the planar, axisymmetric and spherically symmetric flame annihilation from DNS (solid line), zero flame thickness (dash-dotted). Talei et al., 2011 
2.7 Comparison of DNS (solid) and solution of Lighthill's equation (dashed) at an instant after annihilation (i) obtaining propagation velocity from the simulation results and (ii) using the Markstein length theory. [Talei et al., 2012a] . . . . . . . . . . . 22

2.8 Dimensionless dilatation field for forced, laminar, 2D flames for $S t_{D}=0.025$ and (a) $L e=0.5$, (b) $L e=1$ and (c) $L e=2$. (Talei et al. $[2012 b]) \ldots \ldots \ldots \ldots \ldots$

3.1 Schematic of the computational domain (not to scale). Note that the coordinate origin is located at the jet centreline on the inflow plane.

3.2 (a) Normalised temperature $\theta$ (solid) and reactant species mass fraction $\left(Y_{R}\right)$ (dashed) profiles at the inlet boundary; (b) The mean inlet streamwise velocity $\left(\bar{U}_{x} / \bar{U}_{c}\right)$ profile (solid) and the normalised RMS of streamwise velocity fluctuations $\left(u_{r m s}^{\prime} / \bar{U}_{c}\right)$ (dashed) at the inlet boundary. . . . . . . . . . . . . . . 37

3.3 The procedures of determining the correct magnitudes of Da (Damköhler number) and $R e$ (acoustic Reynolds number) to achieve the target values of $S_{L}$ and $\delta_{t h}$ in S3D-SC. . . . . . . . . . . .

3.4 The inlet spectrum (solid line) of streamwise turbulent kinetic energy $\left(u^{\prime 2}\right)$ at the jet centreline. Note that the dashed line depicts the well-known $f^{-5 / 3}$ law for the kinetic energy spectrum in the inertial subrange. . . . . . . . . . . . . . . . . . 41

3.5 Grid stretching ratio in the transverse directions. . . . . . . . . 41

3.6 Dilatation field on the jet central plane for four different mesh resolutions (grid points per jet diameter): (a) 114, (b) 142, (c) 185 and (d) 200. Occurrences of numerical noise are indicated. . . . .

3.7 (a) An instantaneous snapshot of the normalised temperature field $\theta$ at the plane $z=0$. (b) $\theta$ interpolated along the lines A-E indicated in (a) . . . . . . . . . . . . . . . .

3.8 Comparison of the dimensionless dilatation field $\nabla \cdot \vec{u}$ at the jet central plane (a) without and (b) with the sponge layer. . . . . 45 
3.9 Comparison of the dimensionless dilatation field $\nabla \cdot \vec{u}$ (left) and the streamwise velocity field $U_{x}$ (right) at two different axial locations without (solid line) and with (circle) the sponge layer implemented: (a) close to the start of the sponge layer and (b) $2 D$ upstream of the start of the sponge layer. . . . . . . . . . . . .

3.10 (a) The normalised time history of (a) the minimum (thin solid) and the maximum (thick solid) pressure in the whole computational domain; and (b) the volume mean of reactant species mass fraction $\left(Y_{R}\right)$. The result of the stoichiometric case is shown here.

4.1 The locations of the present DNS cases on the regime diagram for premixed turbulent combustion: $\phi=1.0(\bullet)$ and $\phi=0.7(\square)$. .

4.2 Instantaneous snapshots of (i) the flame iso-surface (ii) the temperature field $T$ and (iii) the streamwise velocity field $U_{x}$ extracted at the central plane through the jet: $\phi=1.0$ (left) and $\phi=0.7$ (right). 51

4.3 The time averaged (i) temperature field $\bar{\theta}$ and (ii) streamwise velocity field $\bar{U}_{x}$ at the jet central plane: (a) $\phi=1.0$ and (b) $\phi=0.7$. 52

4.4 Jet half-width $r_{1 / 2}(x): \phi=1.0$ (solid) and $\phi=0.7$ (dashed) . . . 553

4.5 (a) Time averages and (b) RMS of the fluctuations of (i) the normalised temperature field and (ii) the normalised streamwise velocity field conditioned on $r / D$ at three different streamwise locations: $x / D=2$ (solid line $\phi=1.0$ and solid circle $\phi=0.7$ ), $x / D=10$ (dashed $\phi=1.0$ and hollow circle $\phi=0.7$ ) and $x / D=18$ (dash-dot

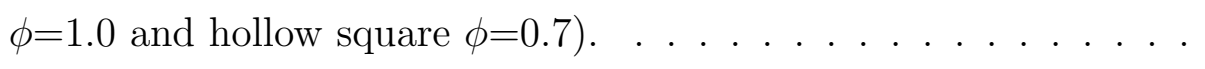

4.6 Instantaneous iso-surfaces of the progress variable $Y_{R}=0.122$ and $Y_{R}=0.156$ for (a) $\phi=1.0$ and (b) $\phi=0.7$, respectively. . . . . . .

4.7 Instantaneous snapshots of the dimensionless dilatation field $\nabla \cdot \vec{u}$ on the jet central plane for $\phi=1.0$.

4.8 Instantaneous snapshots of the dimensionless dilatation field $\nabla \cdot \vec{u}$ on the jet central plane for $\phi=0.7 \ldots \ldots \ldots \ldots$ 
4.9 Instantaneous iso-surface with $Y_{R}=0.122$ superimposed on the dilatation field for the three labelled events A1, A2 and A3 in figure 4.7. The dashed circle shows the monopolar sound wave generated by each annihilation event. . . . . . . . . . 59

4.10 (i) Time-averaged reaction rate; (ii) variance of reaction rate; (iii) horizontally integrated variance field normalised by its maximum value (dashed: $\mathrm{x}-\mathrm{y}$ plane, dash-dot: $\mathrm{x}-\mathrm{z}$ plane and solid: the average of these planes) for (a) $\phi=1.0$ and (b) $\phi=0.7$. . . . . . . . . 61

4.11 Thirty two virtual probes located at three different axial locations $x / D=2,10$ and 18 (a), distributed uniformly on a circle with $r / D=6$ at each particular $x / D$ location $(\mathrm{b}) . \ldots \ldots . . .$.

4.12 Noise spectrum for $\phi=1.0$ (solid) and $\phi=0.7$ (dashed) averaged over 32 equally spaced probes at $x / D$ : (a) 18, (b) 10 and (c) 2. The horizontal axis shows Strouhal number based on flame length $\left(S t_{L_{\text {flame }}}=f L_{\text {flame }} / U_{a v}\right)$. The vertical axis shows the sound

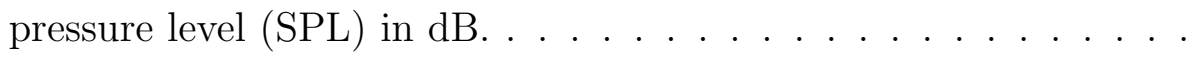

4.13 RMS of the acoustic pressure $\left(p_{\text {rms }}^{\prime}\right)$ for $\phi=1.0(\bullet)$ and $\phi=0.7$ ( $\square$ ) considering thirty two virtual probes at $r / D=6$ and three different axial locations $x / D$ : (a) 18 , (b) 10 and (c) $2 \ldots \ldots$. . .

5.1 The time evolution of the three flame annihilation events labelled in figure 4.7 and their corresponding normalised density weighted flame displacement speed $\left(S_{d}^{*} / S_{L}\right)$ fields mapped on the flame surface.

5.2 Average reaction rate and scatter plot of nodes with $S_{d}^{*} / S_{L}>10$ on an iso-surface with a) $Y_{R}=0.122$ for $\phi=1.0$ and b) $Y_{R}=0.156$ for $\phi=0.7$. The stoichiometric flow through time resulting from table 3.1 was used to produce plots.

5.3 Normalised density weighted flame displacement speed $\left(S_{d}^{*} / S_{L}\right)$ as a function of normalised flame radius $\left(r_{0} / \delta_{t h}\right)$ for a spherically symmetric annihilation event for $\phi=1.0$ (solid) and $\phi=0.7$ (dashed). 
5.4 Time history of sound generated by a spherically symmetric annihilation event at $r / \delta_{t h}=28$ from DNS for $\phi=1.0$ (solid) and $\phi=0.7$ (dashed) and by equation 5.3 for $\phi=1.0$ (square) and $\phi=0.7$ (circle) . . . . . . . . . . . . . . . . 73

5.5 (a) Comparison of the sound generated by a spherically symmetric annihilation event at $r / \delta_{t h}=28$ using equation 5.3 (solid) and the modified theory (equation 5.5) with $n=2.5$ (dashed), 10 (dashdot) and 20 (dotted). (b) The corresponding time history of $S_{d}^{*} / S_{L}$ for this event. . . . . . . . . . . . . . . .

5.6 (a) One snapshot of the flame iso-surface for $\phi=1.0$ on a uniform mesh with a cube of edge length $\Delta=2 \delta_{t h}$. (b) The regions B1, B2 and B3 in (a) are zoomed in and coloured with the $S_{d}^{*} / S_{L}$ field. (c) Centroid of the cluster of the conditioned data as per section 5.3.3.2. 766

5.7 Time history of the far-field noise at $r=6 D$ and $x=10 D$ for $\phi=1.0$, obtained from (a) DNS, (b) model with $n=10$ and (c) model with $n=5$.

5.8 Correlation coefficient $\rho$ between the DNS far-field noise and that of the model at $r=6 D$ and $x=10 D$ as a function of $S_{d}^{*} / S_{L}$ for $\phi=1$ (solid circles) and $\phi=0.7$ (hollow circles) . . . . . . . . . . . . .

5.9 Distribution of the correlation coefficient $\rho$ between the DNS and the model calculated over a $x-z$ plane located at $y / D=-6$ for (a) $\phi=1.0$ with $n=5$ and (b) $\phi=0.7$ with $n=12.5 \ldots \ldots$. . . .

6.1 The instantaneous distribution of different terms of the normalised density weighted flame displacement speed ( (i) net component, (ii) reaction component, (iii) normal diffusion component and (iv) curvature component) over the flame surfaces of three distinct annihilation events: (a) 'island burn-out', (b) 'pinch-off' and (c) 'tunnel formation' events. . . . . . . . . . . . . . . . . . 
6.2 Scatter plots of different terms of the normalised density-weighted flame displacement speed ( (solid circle) net component, (hollow circle) reaction component, (solid square) normal diffusion component and (hollow square) curvature component) versus the normalised curvature $\left(\delta_{t h} \bullet \nabla . \vec{n}\right)$ over the flame surfaces of the three distinct annihilation events shown in figure 6.1: (a) 'island burnout', (b) 'pinch-off' and (c) 'tunnel formation' events. . . . . . . .

6.3 PDFs of the normalised curvature $\delta_{t h} \bullet \nabla . \vec{n}$ for (a) entire flame and (b) considering only annihilation events: $\phi=1.0$ (solid) and $\phi=0.7$ (dashed) . . . . . . . . . . . . . . . .

6.4 PDFs of flame displacement speed components for $\phi=1.0$ (solid) and $\phi=0.7$ (dashed) over entire flame: (a) net component, (b) reaction component, (c) normal diffusion component and (d) curvature component. . . . . . . . . . . . . . . .

6.5 Conditional mean of percentage contribution of the absolute values of different terms of $S_{d}^{*} / S_{L}$ versus the normalised curvature for entire flame ((solid line) reaction component, (dashed) normal diffusion component and (dash-dot) curvature component)) and for annihilations events ((solid circle) reaction component, (hollow circle) normal diffusion component and (solid square) curvature component): (a) $\phi=1.0$ and (b) $\phi=0.7 \ldots \ldots \ldots \ldots$

6.6 Correlation of different terms of the normalised density-weighted flame displacement speed $\left(S_{d}^{*} / S_{L}\right)$ with normalised curvature $\left(\delta_{t h} \bullet\right.$ $\nabla . \vec{n})$ for entire flame $(\phi=1.0(\mathrm{a})$ and $\phi=0.7(\mathrm{~b}))$ and considering only annihilation events ( $\phi=1.0$ (c) and $\phi=0.7$ (d)): i) net term, ii) reaction term and iii) normal diffusion term. Dashed lines show curvature conditional means of $S_{d}^{*} / S_{L}$ terms . . . . . . . .

6.7 The joint PDFs with normalised curvature $\left(\delta_{t h} \bullet \nabla . \vec{n}\right)$ of (i) reaction rate $\dot{\omega}_{R}$ and (ii) the magnitude of reactant mass-fraction gradient $\left|\nabla Y_{R}\right|$ for the case with $\phi=1.0$ : (a) entire flame and (b)

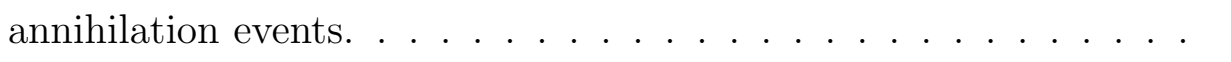


6.8 Correlation coefficient between different terms of the normalised density weighted flame displacement speed ((a) net term, (b) reaction rate term and (c) normal diffusion term) and the normalised curvature conditioned on the streamwise direction $x / D$ and considering only annihilation events for $\phi=1.0$ (solid) and $\phi=0.7$ (dashed) . . . . . . . . . . . . . . . .

6.9 PDFs of the normalised flame stretch for $\phi=1.0$ (solid) and $\phi=0.7$ (dashed) with no condition on $S_{d}^{*} / S_{L}$ : (a) $K a$, (b) $K a_{S}$ and (c) $K a_{C} \ldots \ldots \ldots \ldots \ldots \ldots \ldots \ldots \ldots$

6.10 PDFs of the normalised flame stretch for (a) $\phi=1.0$ and (b) $\phi=0.7$ considering only the annihilation events: (i) $K a$, (ii) $K a_{S}$ and (iii) $K a_{C} \ldots \ldots \ldots \ldots \ldots \ldots \ldots \ldots$

6.11 Correlation of the normalised flame stretch $(K a)$ with $S_{d}^{*} / S_{L}$ for (a) $\phi=1.0$ and (b) $\phi=0.7$ : (i) whole flame surface statistics and (ii) considering only the annihilation events. The dashed lines show the $K a$ conditional mean of $S_{d}^{*} / S_{L} \ldots \ldots \ldots \ldots$

6.12 Correlation between $K a$ and $S_{d}^{*} / S_{L}$ for annihilation events from DNS (scatter), original theory (equation 6.9) (solid line) and new proposed linear model (equation 6.11) (dashed line): (a) $\phi=1.0$ and $(\mathrm{b}) \phi=0.7 \ldots \ldots \ldots \ldots \ldots$

6.13 Time history of the far-field noise at $r=6 D$ and $x=10 D$ for $\phi=1.0$, obtained from the simple model proposed in chapter 5 with different terms of $S_{d}^{*} / S_{L}$ contributing to $V_{f}$. (a) Excluding the gas velocity term (u.n): reaction term (solid), normal diffusion term (dashed) and curvature term (dash-dot). (b) Including the gas velocity term (u.n): net term (solid) and reaction term (dashed). 100

6.14 Time history of the far-field noise at $r=6 D$ and $x=10 D$ obtained from our simple model with $V_{f}=\mathbf{u . n}+S_{d_{\mathrm{DNS}}}$ (solid) and with $V_{f}=\mathbf{u . n}+S_{d_{\text {model }}}$ (dashed) for (a) $\phi=1.0$ and (b) $\phi=0.7 . \ldots 101$ 


\section{List of Tables}

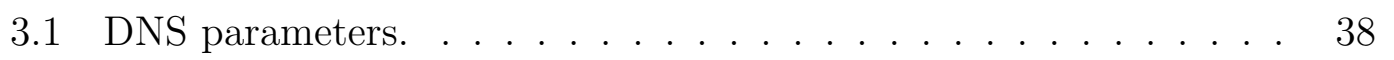

6.1 Calculated Markstein numbers from (a) turbulent premixed flames under no condition, spherically symmetric flame $\left(r_{0} / \delta_{t h}>2\right)$ and theory (Eq6.7) and (b) considering only turbulent premixed flame annihilation events. . . . . . . . . . . . . . 98 


\section{Nomenclature}

\section{Roman Symbols}

$\dot{m}_{f} \quad$ mass flow rate of fuel

n unit normal vector

q heat flux vector

$\mathbf{u} \quad$ flow velocity vector

$A \quad$ flame surface area

$a_{T} \quad$ tangential strain rate

$B \quad$ pre-exponential factor

c sonic velocity

$D \quad$ jet diameter

$D_{m} \quad$ binary mass diffusion coefficient

$D_{t h} \quad$ thermal diffusivity coefficient

Da Damköhler number

$E_{a} \quad$ activation energy

$E_{t} \quad$ total energy per unit mass

$f \quad$ frequency 
$f \quad$ mass burning flux $=\rho_{u} S_{L}$ (equation 3.32

$H \quad$ Heaviside function

$H \quad$ fuel heating value (equation 2.14)

$h_{k}^{0} \quad$ chemical enthalpy of formation

Ka Karlovitz number

$K a_{C}$ curvature component of $K a$

$K a_{S}$ tangential strain rate component of $K a$

$L \quad$ domain size

$l_{11} \quad$ longitudinal integral length scale

$l_{22,33}$ transverse integral length scales

$l_{F} \quad$ characteristic laminar flame thickness

$L_{k} \quad$ amplitudes of different characteristic waves

$l_{M} \quad$ Markstein length scale

$l_{T} \quad$ characteristic length scale of turbulence

Le Lewis number

M mixture molecular weight

$M_{k} \quad$ species molecular weight

Ma Markstein number in terms of $S_{d}^{*}$

Ma Markstein number

$P \quad$ total acoustic power

$p \quad$ pressure

Pr Prandtl number 
$Q \quad$ heat of reaction per unit mass of reactant

$R \quad$ ideal gas constant

$r \quad$ distance between receiver and sound source

$r_{0} \quad$ flame radius

$r_{1 / 2}(x)$ jet half-width

Re acoustic Reynolds number

$R e_{D}$ jet Reynolds number

$S_{d} \quad$ flame displacement speed

$S_{d}^{*} \quad$ density-weighted flame displacement speed

$S_{L} \quad$ laminar flame speed

Sc Schmidt number

St Strouhal number

T temperature

$t \quad$ time

$T_{f} \quad$ adiabatic flame temperature

$U \quad$ jet mean velocity

$V_{f} \quad$ flame propagation velocity

$x \quad$ Cartesian coordinates

$Y_{R} \quad$ reactant mass fraction (progress variable)

\section{Greek Symbols}

$\alpha \quad$ heat release parameter

$\beta \quad$ Zel'dovich number 
$\Delta \quad$ cube edge length

$\delta_{f} \quad$ nominal flame thickness

$\delta_{i j} \quad$ Kronecker delta

$\delta_{t h} \quad$ laminar flame thickness

$\dot{\omega}_{R} \quad$ reactant species reaction rate

$\eta \quad$ Kolmogorov length scale

$\gamma \quad$ heat capacity ratio

$\kappa \quad$ stretch

$\lambda \quad$ mixture thermal conductivity

$\lambda_{k} \quad$ characteristic velocities

$\mu \quad$ dynamic viscosity

$\nabla . n \quad$ flame curvature

$\nu \quad$ kinematic viscosity

$\phi \quad$ equivalence ratio

$\rho \quad$ mixture density

$\tau_{i j} \quad$ viscous stress tensor

$\tau_{j} \quad$ jet flow-through time

$\theta \quad$ normalised tempreture

\section{Superscripts}

()$^{*}$ dimensional value

()$^{\prime} \quad$ Fluctuating value

() Averaged value 


\section{Subscripts}

()$_{\infty} \quad$ far-field value

()$_{b} \quad$ burned gas value

()$_{c} \quad$ curvature component

()$_{D} \quad$ deficient reactants

()$_{E} \quad$ excess reactants

()$_{n} \quad$ normal diffusion component

()$_{\text {ref }}$ reference value at the jet inlet

()$_{r m s}$ root-mean-square value

()$_{r} \quad$ reaction component

()$_{u} \quad$ unburned gas value 


\section{Chapter 1}

\section{Introduction}

\subsection{Motivation}

In addition to being a significant form of noise pollution, combustion-generated noise has an additional importance because it hampers the development of lowemission, premixed combustors in modern gas turbines. This is because these types of combustors are susceptible to so called 'thermoacoustic instability' that is associated with the sound generated by the flame inside the combustor. The interaction of the reflected acoustic waves from the combustor's walls and nozzle with the flame can trigger instability and produce strong pressure fluctuations, leading to engine failure in extreme cases (see figure 1.1).

The University of Melbourne group has recently studied so-called 'flame annihilation events', and these appear to be a significant source of sound. However, the significance of these events in turbulent premixed flames has not been studied. This research therefore intends to bring insight into the mechanisms of sound generation by turbulent premixed flames using Direct Numerical Simulation (DNS), with the simulations used to examine the contribution of flame annihilation events to the overall produced sound. 
(a)

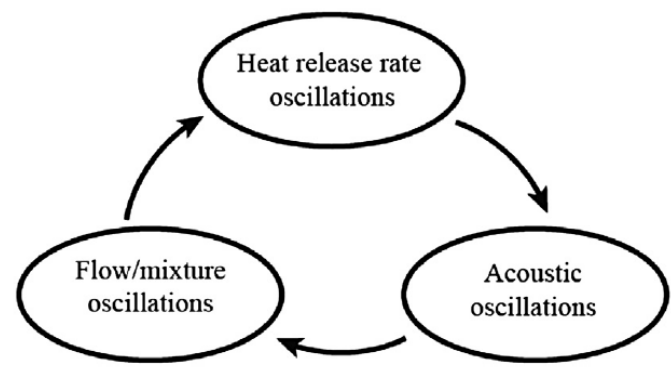

(b)

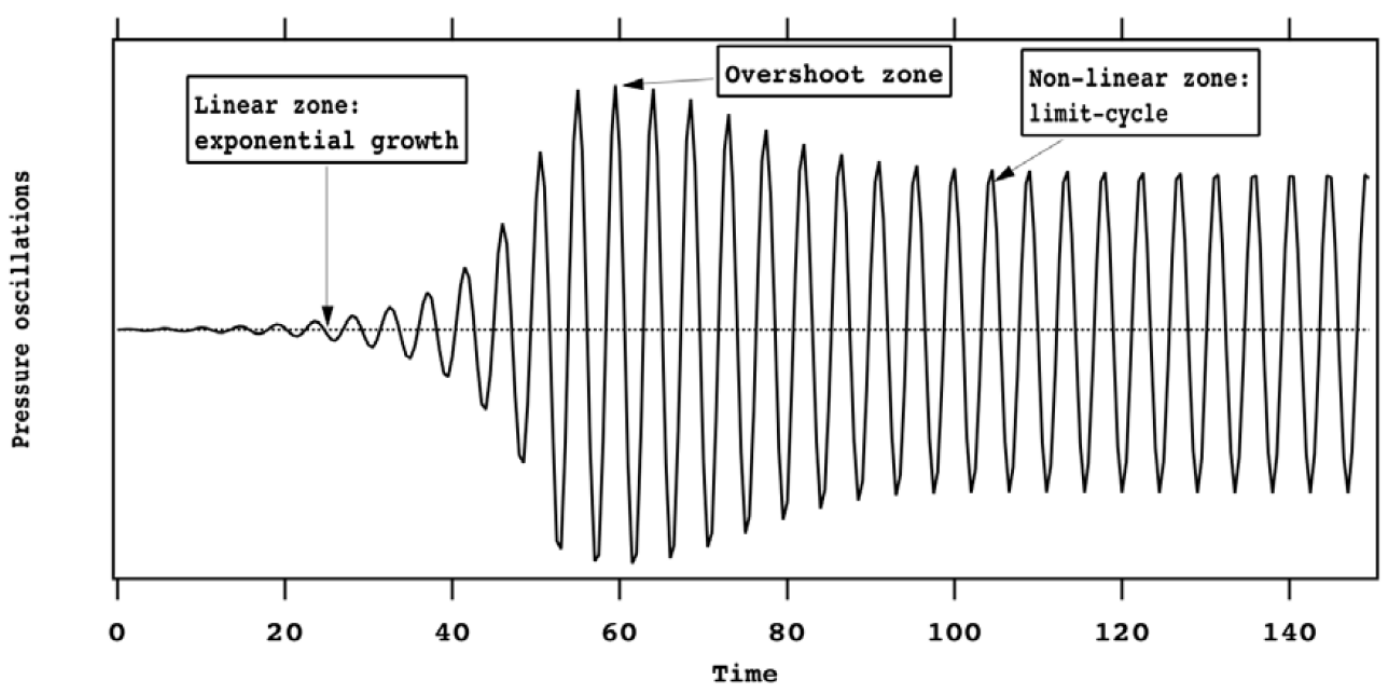

Figure 1.1: (a) Thermoacoustic feedback cycle [O'Connor et al., 2015]; (b) Typical time history of pressure signal associated with a combustion instability Poinsot \& Veynante, 2005.

\subsection{Research Aims}

There are three research aims for this thesis.

I) To perform DNS of sound generation by turbulent premixed flames.

A three dimensional (3D) DNS study of sound generation by open turbulent premixed flames is performed. A single step chemistry model for methane/air combustion is used. Two cases with different equivalence ratios $(\phi=0.7$ and 1.0) and preheated unburned mixture of $T_{u}=800 \mathrm{~K}$ are simulated. The reactant 
temperature is representative of modern gas turbines. Chapter 4 presents main findings including the contribution of flame annihilation events to the emitted noise.

II) To examine the contribution of so-called "flame annihilation" events to the overall produced sound.

Chapter 5 presents a simple model that determines the contribution of annihilation events to the overall produced sound. First, flame annihilation events are identified using the flame displacement speed as a marker since high values of displacement speed are observed during flame annihilation. It is then assumed that all annihilation events in a turbulent flame are spherically symmetric. Using a modified form of the existing theory of Talei et al. $[2012 a]$ the noise generation by annihilation events throughout the flame is then obtained. Finally, the DNS and the model predictions are compared, highlighting the significance of flame annihilation events to the overall produced sound by turbulent premixed flames.

III) To examine the flame response to strain and curvature during flame annihilation.

It is known from the literature that the flame response to instabilities are modified by strain and curvature. The significance of different terms contributing to the flame displacement speed and their correlation with curvature are explored. Correlation of the flame displacement speed with flame stretch is also studied. The Markstein number is then calculated and a new linear model for response of turbulent premixed flames to stretch during flame annihilation is proposed. Finally, the contribution of different terms of the flame displacement to the sound produced by turbulent premixed flames is also evaluated through the simple model previously proposed in chapter 5 . 


\section{Chapter 2}

\section{Literature Review}

\subsection{Introduction}

To minimise noise from devices such as internal combustion engines and industrial burners, it is important to have a reasonable understanding of sound generation by combusting flows. Combustion noise is generally classified as either 'direct' or 'indirect'. Direct noise is generated by the flame whereas indirect noise is due to flow inhomogeneity downstream of the flame. Indirect combustion noise is not reviewed in this chapter because it is beyond the scope of this thesis.

In addition to being a significant form of noise pollution, sound generation in reacting flows can also cause combustion instability which in turn can have an adverse impact on combustion system performance. In extreme cases, this instability may lead to the mechanical failure of the combustion chamber. Hence, understanding the mechanisms of sound production by combusting flows can also help us design cleaner combustion devices, benefiting humans, animals and the environment more broadly.

Despite dedicating more than half a century to the investigation of combustion noise, significant aspects of this problem are not yet fully understood. However, the recent developments in high performance computing provide an opportunity to bring valuable insight into the sources of combustion noise. 


\subsection{Fundamentals of Combustion Noise}

In one of the earlier studies of combustion noise, Smith \& Kilham [1963] experimentally investigated sound generation by open turbulent premixed flames. They showed that the emitted sound originates from a distribution of monopolar sources throughout the flame. It was also found that combustion noise features a broadband spectrum where the frequency of its peak amplitude may be related to the burner diameter, the flow velocity and the flame speed. Smith \& Kilham [1963 also conducted a scaling analysis for the total acoustic power, integrating the acoustic intensity over a given surface in the far field. The relationship between the total acoustic power and the flow parameters of a premixed turbulent flame was expressed as $P \propto\left(U D S_{L}\right)^{2}$. Based on this scaling they concluded that the sound production by a non-combusting flow is negligible in comparison with a combusting jet with the same flow parameters.

Bragg [1963] established a simple theory to describe the relationship between the sound production and combustion by assuming that the generated noise was the result of changes in the flame volume, which was assumed to be directly proportional to changes in the heat release rate. Many of the subsequent theoretical studies of sound generation by turbulent premixed flames have also attempted to relate the radiated sound to changes in the flame volume or the heat release rate. Indeed, heat release rate fluctuations have been demonstrated to be significant in many studies of sound generation by premixed flames e.g., Candel et al. 2004); Dowling 1992; Hurle et al. [1968; Kotake \& Takamoto 1990; Liu 2015]; Rajaram \& Lieuwen 2009]; Schuller et al. [2003]; Strahle [1978]; Swaminathan et al. 2011a b]. Based on his model, Bragg [1963] also pointed out that the noise emission will be intensified by increasing the reactivity of the fuel and the flow velocity in the system. In addition, it was shown that one millionth of the fuel energy is converted to the combustion noise energy. This is two orders of magnitude greater than that of a non-reacting jet at similar conditions.

Thomas \& Williams 1966 examined sound generation by a spherically symmetric premixed fuel-air mixture. They used a soap bubble containing the premixture of fuel and air and then ignited it centrally. It was found that the ratio of the produced sound energy to the thermal input was about $10^{-6}$ for typi- 
cal hydrocarbon-air flames, consistent with Bragg's [1963 finding. Thomas \& Williams [1966 also presented a theory to calculate sound emission in the far field, showing a good agreement with the measured radiated sound. The theory assumes that the flame is a monopolar source of noise with the sound emission depending on the flame surface acceleration/deceleration. Subsequently, experimental studies by Hurle et al. [1968] and Price et al. [1969] demonstrated that this theory can be extended for turbulent flames by approximating the combustion zone with a collection of monopolar sources, showing good agreement with experimental results.

Briffa et al. [1973] conducted a series of experiments to investigate the sound emission by premixed and diffusion jet flames. In addition to confirmation of the previous findings, they observed that premixed flames produced more noise compared with diffusion flames for a given thermal output.

One mechanism that causes rapid changes of the flame volume (and heat release rate consequently) in premixed flames is flame annihilation (Kidin et al. [1984], Kidin et al. [1988], and Candel et al. [2004]). This mechanism does not exist in diffusion flames, which could be the reason for premixed flames being noisier than non-premixed flames as reported by Briffa et al. [1973]. Flame annihilation can occur when two flame surfaces collide with each other so that a rapid consumption of the unburned gas trapped between these surfaces is observed, leading to large fluctuations of the heat release rate and therefore a high level of produced noise.

These events were first reported by Kidin et al. [1984] as strong monopolar sources of sound in premixed flames. They experimentally studied a conical jet flame excited by a loudspeaker at the base, providing a periodic variation in the inlet flow velocity. They measured the far-field pressure and observed two peaks in the pressure history. These two sudden changes in the pressure trace were correlated with two types of annihilation events observed during the experiment. The first event was the collapse of the 'flame neck', and the second was the consumption of a 'pocket' of unburned gas shed by the first event. Some recent results have demonstrated the important role played by annihilation events in combustion noise, which will be addressed later in detail. 


\subsection{Lighthill's Acoustic Analogy}

Acoustic analogies are a rearrangement of the Navier-Stokes equations into various forms of an inhomogeneous acoustic wave equation. The right hand side of this wave equation features a number of source terms, which can potentially represent different physical mechanisms for generation of sound. These acoustic analogies are commonly used to shed light on the mechanism of sound generation by different flow configurations.

The analogy proposed by Lighthill [1952, 1954 is the first and best-known acoustic analogy. Lighthill combined and rearranged the exact conservation equations of mass and momentum into an inhomogeneous wave equation,

$$
\frac{\partial^{2} \rho^{\prime}}{\partial t^{2}}-c_{0}^{2} \nabla^{2} \rho^{\prime}=\frac{\partial^{2} T_{i j}}{\partial x_{i} \partial x_{j}}
$$

where

$$
T_{i j}=\rho u_{i} u_{j}+\delta_{i j}\left[\left(p-p_{0}\right)-c_{0}^{2}\left(\rho-\rho_{0}\right)\right]-\tau_{i j} .
$$

The term $T_{i j}$ is known as Lighthill's stress tensor. The first term in $T_{i j}$ is the Reynolds stress tensor. It is a quadrupolar distribution of sound sources, and is a significant source of sound in non-reacting, turbulent flows (Figure 2.1). The second and third terms are the contributions of entropy variations and viscous stresses inside the source region in producing sound. For low Mach number, unheated flows, $T_{i j} \approx \rho_{0} u_{i} u_{j}$ where $\rho_{0}$ is the mean density of the medium. Equation 2.1 then shows that the flow-generated sound can be viewed as the acoustic emission by a distribution of quadrupole sources with the strength $T_{i j}$ into a stationary, ideal fluid. The solution to this equation can be obtained using Green's functions (c.f. Duffy 2001),

$$
\rho(\mathbf{x}, t)-\rho_{0}=\rho^{\prime}(\mathbf{x}, t)=\frac{1}{4 \pi c_{\infty}^{4}} \frac{\partial^{2}}{\partial x_{i} \partial x_{j}} \int T_{i j}(\mathbf{y}, \tau) \frac{d \mathbf{y}}{|\mathbf{x}-\mathbf{y}|}
$$

where $\tau$ is the retarded time, 


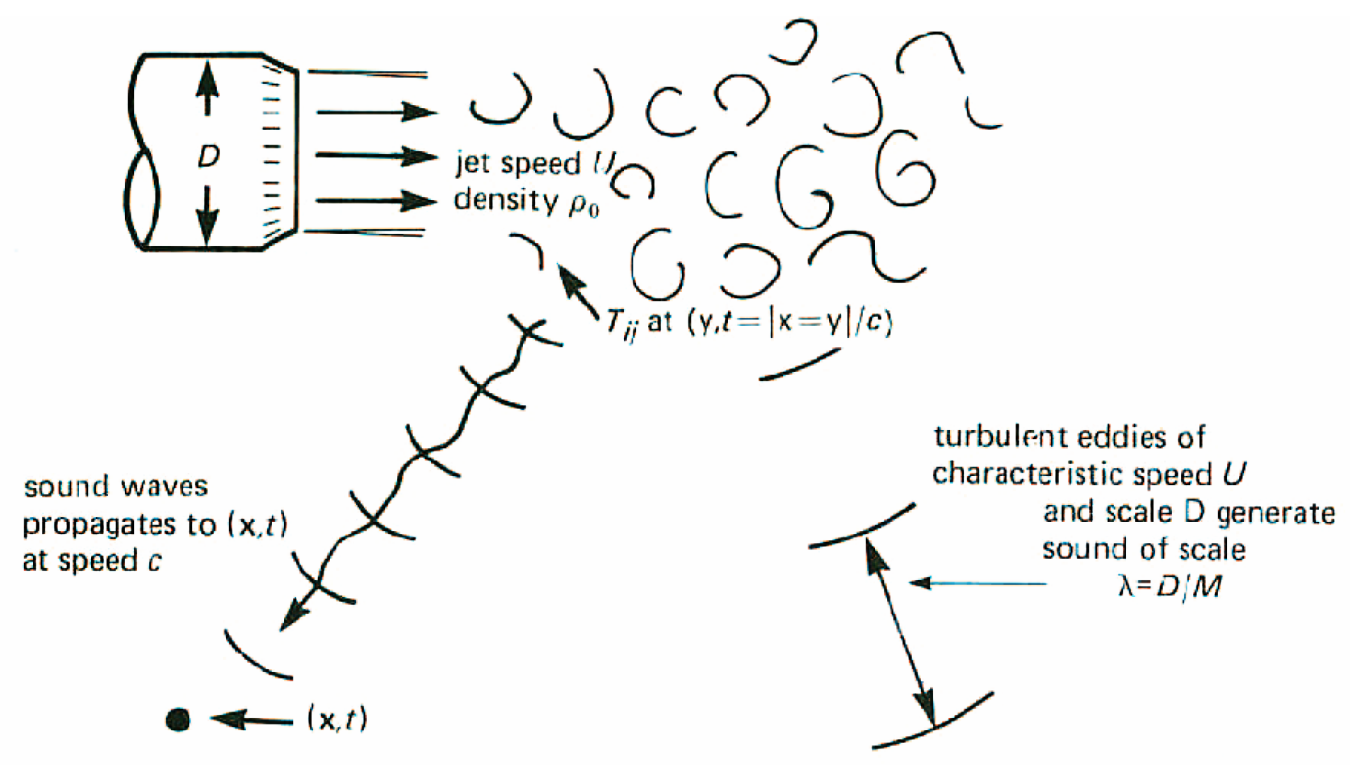

Figure 2.1: Schematic of sound radiation by a low Mach number jet (Dowling \& Williams [1983]).

$$
\tau=t-|\mathbf{x}-\mathbf{y}| / c_{\infty}
$$

In the far field where $x=|\mathbf{x}| \gg|\mathbf{y}|$ (see figure 2.1), equation 2.3) becomes,

$$
\rho^{\prime}(\mathbf{x}, t)=\frac{1}{4 \pi c_{\infty}^{4}} \int \frac{x_{i} x_{j}}{x^{3}} \frac{\partial^{2} T_{i j}}{\partial \tau^{2}}(\mathbf{y}, \tau) d \mathbf{y}
$$

Using scaling arguments, Lighthill [1952] also showed that the acoustic intensity - acoustic energy flux per unit area - radiated from a low Mach number jet is proportional to the "eighth power" of the jet exit velocity. Westley \& Lilley 1952 conducted a comprehensive experimental investigation and confirmed Lighthill's "eighth-power law". 


\subsubsection{Combusting Forms of Lighthill's Acoustic Analogy}

Strahle [1971] used Lighthill's acoustic analogy to examine sound generation by combusting jets. Strahle 1971 divided the Lighthill's source term into three parts, $\partial T_{i j} / \partial x_{i} x_{j}=F_{1}+F_{2}+F_{3}$, where

$$
F_{1}=\left(\rho u_{i} u_{j}\right)_{x_{i} x_{j}}, F_{2}=p_{x_{i} x_{j}} \text { and } F_{3}=-c_{\infty}^{2} \rho_{x_{i} x_{j}} .
$$

He then argued that although $F_{1}$ was considered by Lighthill to be the dominant source of sound in cold jets, this term does not play a major role in combusting jets (e.g., Smith \& Kilham [1963], Kotake \& Hatta 1965], and Smithson \& Foster 1965). In addition, no directionality of a quadrupole-shape acoustic field characterised by the first term is observed in combustion noise experiments. Assuming a locally laminar mechanism for flame propagation inside the turbulent region, he elucidated that $F_{2} / F_{3}$ is proportional to $S_{L}^{2} / c_{\infty}^{2}$ which is approximately of the order of $10^{-6}$. Hence, the solution of Lighthill's inhomogeneous wave equation was given by the term $F_{3}$ only,

$$
\rho^{\prime}(\mathbf{x}, t)=-\frac{1}{4 \pi c_{\infty}^{2} r} \frac{\partial^{2}}{\partial t^{2}} \int_{V} \rho\left(\mathbf{y}, t-\frac{r}{c_{\infty}}\right) d \mathbf{y} .
$$

The total acoustic power was then calculated using equation 2.7,

$$
P=\frac{1}{4 \pi \rho_{u} c_{\infty}} \int_{V} d \mathbf{y} \int_{V_{c o r}} d \epsilon\left[\overline{\rho_{t t}\left(\mathbf{y}, t-\frac{r}{c_{\infty}}\right) \rho_{t t}\left(\mathbf{y}+\epsilon, t-\frac{r}{c_{\infty}}\right)}\right]
$$

Strahle used equation 2.8 as a basis for providing estimations of the total acoustic power as a function of the flow/flame parameters such as $U, S_{L}$ and $l_{T}$ ( Strahle, 1971, 1972, 1978, 1985).

Hassan 1974 used a modified form of Lighthill's acoustic analogy previously put forward by Doak 1972, 


$$
\begin{aligned}
\frac{1}{c_{\infty}^{2}} \frac{\partial^{2} p}{\partial t^{2}}-\nabla^{2} p= & \left(\frac{1}{c_{\infty}^{2}}-\frac{1}{c^{2}}\right) \frac{\partial^{2} p}{\partial t^{2}}-\frac{\gamma-1}{c^{2}} \frac{\partial}{\partial t} \sum h_{k} W_{k}+\frac{\partial}{\partial x_{i}}\left[\frac{\partial}{\partial t}\left(\rho_{e} u_{i}\right)\right] \\
& +\frac{\partial^{2}\left(\rho u_{i} u_{j}\right)}{\partial x_{i} \partial x_{j}}+O\left(c^{-3}\right)+\text { higher order terms, }
\end{aligned}
$$

where

$$
\rho_{e}=\rho-\rho_{\infty}-\left(p-p_{\infty}\right) / c_{\infty}^{2}
$$

Note that in the inhomogeneous parts of equation 2.9 three sources of sound may be identified; that is, a monopolar source induced by combustion $\frac{\partial}{\partial t} \sum h_{k} W_{k}$, as well as a dipole term $\frac{\partial}{\partial x_{i}}\left[\frac{\partial}{\partial t}\left(\rho_{e} u_{i}\right)\right]$ and the usual quadrupole term resulting from the Reynolds stress tensor. Hassan 1974 concluded that the dipole term may account for the weak directionality of the sound waves produced by reacting flows. Using equation 2.9 and considering low-Mach-number, lean combustion, Hassan 1974 provided a new scaling highlighting the role of turbulent flame speed, and turbulence intensity and length scales.

Dowling 1992 proposed a slightly different form of equation 2.9 using thermodynamic relations and the conservation equations of energy and species (Appendix A),

$$
\begin{aligned}
\frac{1}{c_{\infty}^{2}} \frac{\partial^{2} p}{\partial t^{2}}-\nabla^{2} p= & \frac{\partial}{\partial t}\left(\frac{\rho_{\infty}}{\rho c_{p} T}\left\{-\left.\sum_{n=1}^{N} \frac{\partial h}{\partial Y_{n}}\right|_{\rho, p, Y_{m}}-\frac{\partial q_{j}}{\partial x_{j}}+\tau_{i j} \frac{\partial u_{i}}{\partial x_{j}}\right\}\right) \\
& +\frac{\partial^{2}}{\partial x_{i} \partial x_{j}}\left(\rho u_{i} u_{j}-\tau_{i j}\right) \\
& +\frac{1}{c_{\infty}^{2}} \frac{\partial}{\partial t}\left\{\left(1-\frac{\rho_{\infty} c_{\infty}^{2}}{\rho c^{2}}\right) \frac{D p}{D t}-\frac{p-p_{\infty}}{\rho} \frac{D \rho}{D t}\right\} \\
& +\frac{\partial^{2}}{\partial x_{i} \partial t}\left(u_{i} \rho_{e}\right) .
\end{aligned}
$$

The right hand side of equation 2.11 consists of several important source terms. The first term is of monopolar nature which is usually thought to be the main 
contributor to combustion noise. The terms in the second line are the contributions of the Reynolds stress tensor and viscous effects. The term in the third line is a dipole source of sound and is of importance if there are regions of unsteady flow with different mean density and sound velocity from the ambient. Dowling showed that this term scales with $M^{8}$. The last term which was also present in Hassan's formulation is important when there is acceleration of density inhomogeneities: the hot spots produced by combustion are accelerated through an inhomogeneous mean flow field generated, for example, by the turbine blade rows.

\subsection{Turbulent Premixed Flames}

In general, there are two different types of combustion depending on whether the fuel and oxidizer are mixed before combustion. When the fuel and oxidizer are completely mixed prior to ignition, so-called 'premixed combustion' takes place. On the other hand, when the fuel and oxidizer are separated and mixed during combustion, a diffusion flame is the result.

In premixed combustion, the flame exits if the fuel-to-air ratio is inside the 'flammability limits'. Although premixed combustion can provide higher efficiency and cleaner combustion, it is more susceptible to unsteady behaviour and instability, resulting in intense pressure fluctuations that in turn can affect combustion performance and even may lead to the failure of the combustion chamber (Swaminathan \& Bray 2011). Hence, understanding sound generation by premixed flames is of particular interest.

\subsubsection{Regime Diagram}

As opposed to laminar combustion, turbulent combustion is characterised by fluctuations of velocity and scalar quantities such as temperature, density and heat release rate. Turbulent premixed flames may be classified by different regimes Borghi, 1985; Peters, 2000 based on the values of

- $u^{\prime} / S_{L}$, i.e. the ratio between the turbulence velocity $\left(u^{\prime}\right)$ and the laminar flame speed $\left(S_{L}\right)$, and 
- $l_{T} / l_{F}$, i.e. the ratio between some characteristic length scale of turbulence $\left(L_{T}\right)$ and the characteristic laminar flame thickness $\left(l_{F}\right)$.

Figure 2.2 illustrates the commonly used regime diagram for premixed turbulent combustion [Peters, 2000]. A brief description of each regime is provided as follows:

\section{- wrinkled flamelets}

This regime is characterised by $u^{\prime}<S_{L}$, where $u^{\prime}$ may be interpreted as the turnover velocity of the large eddies. These eddies cannot deform the flame front sufficiently and therefore although the flame is wrinkled, its propagation is like a laminar flame.

\section{- corrugated flamelets}

In this region, the flame distortion is sufficient to increase its propagation speed compared with a laminar flame. However, the smallest eddies with the size of Kolmogorov length scale are still larger than the flame characteristic length scale and therefore cannot modify the internal structure of the flame.

\section{- thin reaction zones}

In this regime, Kolmogorov eddies can penetrate into the preheat zone of the flame and enhance thermodiffusive processes. Thus, the flame structure in the preheat zone is altered while the reaction zone is still unaffected by turbulence.

\section{- broken reaction zones}

In this regime, the Kolmogorov length scale is much smaller than the laminar flame thickness $\eta / l_{F} \ll 1$. Hence, the eddies of this size can penetrate into the reaction zone and alter the flame inner structure. Consequently, local extinction can occur due to intense straining by turbulent eddies.

The flamelet approach is the basis of many models for turbulent combustion (Poinsot \& Veynante 2005]), considering a turbulent flame brush as an ensemble of discrete laminar flames. This assumption is valid in the regimes of wrinkled-, 


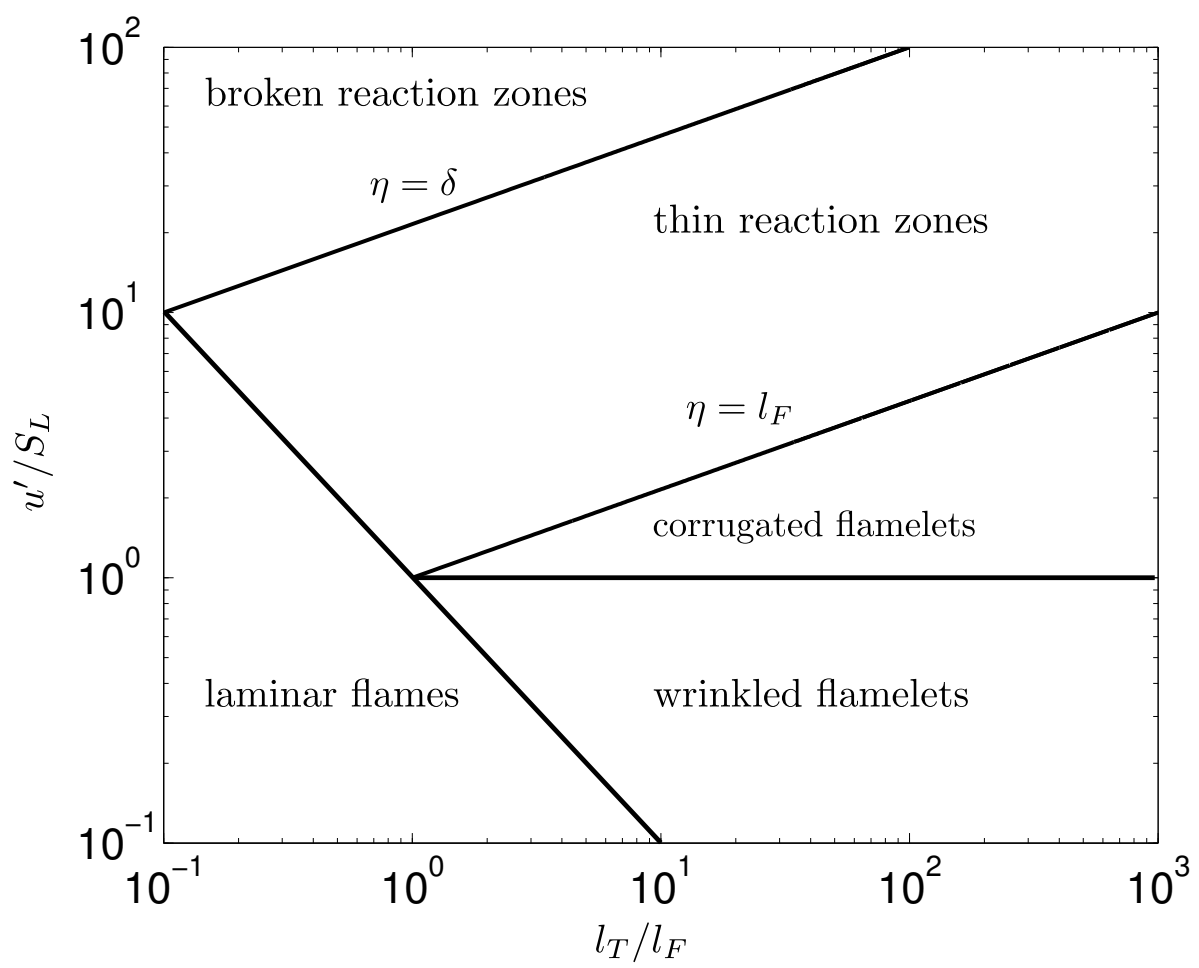

Figure 2.2: The regime diagram for premixed turbulent combustion Peters, 2000].

corrugated-flamelets and thin reaction zones because the fresh mixture and the burned gases are still separated by the thin inner layer of the flame. This is not the case in the broken-reaction zones regime, where local flame extinctions may occur and the gradients along the flame front are large. Note that corrugatedflamelets and thin-reaction zones regime are present in many modern practical combustors [Peters, 2000; Swaminathan \& Bray, 2011]. However, broken-reaction zones regime is of limited interest for practical applications due to high level of pollutions produced by the local extinctions.

\subsubsection{Implications of Regime Diagram for Combustion Noise}

It is plausible to consider a link between different parts of the regime diagram and the generated sound. For example, Strahle [1971] constructed two theories for obtaining scalings of the total acoustic power. He used two models for defining the turbulent flame structure; namely, wrinkled flame theory and distributed 
reaction theory. Using equation 2.8 and the wrinkled flame theory, the total acoustic power was scaled as

$$
P \propto U^{4-3 q} S_{L}^{r+3 q} l_{T}^{2+r}
$$

Strahle 1971 also rearranged equation 2.7 using the continuity equation with the assumption of low Mach number, and presented a scaling analysis for the acoustic power based on the distributed reaction theory,

$$
P \propto U^{2} S_{L}^{3} l_{T}^{3}
$$

As opposed to the total acoustic power obtained from equation 2.13, the total acoustic power based on the wrinkled flame theory (equation 2.12) is dependent on two empirical constants and, as showed by Strahle [1971], this scaling would be in good agreement with experimental data (published by Smith \& Kilham [1963 and Kotake \& Hatta 1965]) if these constants were properly defined.

\subsection{Experimental Studies of Sound Generation by Premixed Flames}

As discussed in section 2.1, early experiential studies have highlighted the important role of heat release rate fluctuation and broadband nature of the combustion noise. Furthermore, studies performed by Kidin et al. 1988, 1984 identified flame annihilation events as a strong source of noise in laminar premixed flames. This finding was again confirmed in a more recent experimental study by Candel et al. 2004 using a similar experimental setup. They carried out three different experimental investigations to study combustion-generated noise in excited laminar flames; namely, interaction of a premixed laminar flame with a cold plate, mutual flame annihilation and flame-vortex interaction. Candel et al. 2004 concluded that the destruction of flame surface area which is accompanied by fluctuations in heat release rate can be regarded as a significant source of sound in unsteady 
(a)

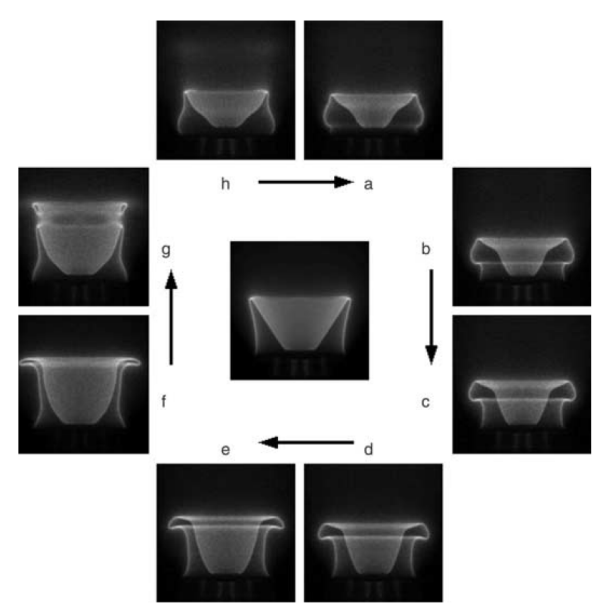

(b)

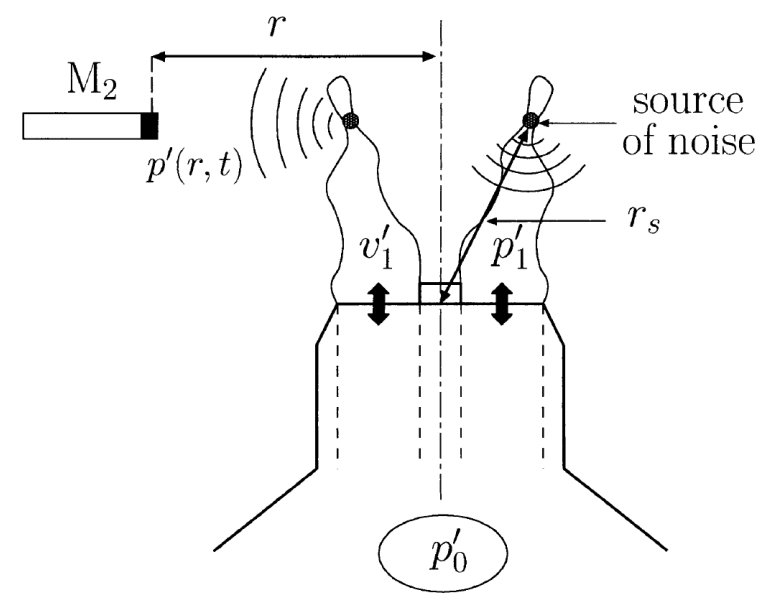

Figure 2.3: (a) View of the "M" stabilised flame: steady flame (in the middle) and unstable flame regime with an oscillation frequency at $f=138 \mathrm{~Hz}$ (a-h). (b) schematic representation of the feedback mechanism leading to self-sustained oscillations [Schuller et al., 2003].

combustion, especially in turbulent reacting flows. In a separate study by the same group, Schuller et al., 2003 observed a strong noise emission by laminar premixed flames stabilised on annular burners ("M" flame, c.f. figure 2.3a). Annihilation events once again were observed as a strong source of noise (see figure $2.3 \mathrm{~b})$ such that they can lead to self-sustained oscillations in these flames.

A comprehensive experimental study of sound generation by turbulent premixed flames was performed by Rajaram \& Lieuwen [2009]. They examined the premixed flame noise spectra in terms of its overall sound pressure level, the frequency of peak emissions, and the dependence of the spectra in the lower and higher frequency ranges. As observed in Figure 2.4, the spectrum features a power-law dependence on the both sides of the peak emission. Rajaram \& Lieuwen [2009] found that the slope of high frequency sideband, $\alpha$, is approximately between 2.2 and 3.6 where $\alpha$ is the slope of high frequency sideband. Wäsle et al. [2005] and Clavin \& Siggia [1991] also reported the value of 8/3 and 2.5 , respectively, for $\alpha$. In the low frequency region of the spectra the slope, $\beta$, is equal to 2 . This behaviour in the low frequency limit was shown to be associated 
with the flame being acoustically a compact source of noise.

The key finding of Rajaram \& Lieuwen 2009] (c.f. figure 2.4 was the dependence of the frequency of peak emissions $f_{\text {peak }}$ on the mean inlet velocity $U_{a v}$ divided by the flame length $L_{\text {flame }}$ across all burners, flow velocities, fuel/air ratios and fuels. They showed that a peak Strouhal number defined as $S t_{\text {peak }}=f_{\text {peak }} L_{\text {flame }} / U_{a v}$ was always of order unity and varied between 0.5 and 2.2 across the range of experimental conditions tested.

Using a simplified version of Dowling's acoustic analogy (Dowling [1992]), Rajaram et al. [2006] derived a straightforward relation for the total acoustic power of combustion noise,

$$
P \propto f_{\text {peak }}^{2}\left(m_{f} \dot{H}\right)^{2}
$$

where the role of the frequency of peak emission is evident. Using equation 2.14 , they achieved an agreement between the theory and experimental results to within $\pm 3.5 \mathrm{~dB}$.

It should be noted that Rajaram \& Lieuwen 2003 and Rajaram 2007 also found that at low equivalence ratios the low frequency noise production was significantly less than that produced by higher equivalence ratios. They suggested that this observation may be attributed to the disappearance of flame tip at low equivalence ratios, and emphasised the need for further study to characterise the key parameters affecting the spectral characteristics of noise generated by turbulent premixed flames.

In a recent study, Tam 2015] used an empirical approach and proposed that combustion noise spectrum is similar to the noise spectrum from large turbulence structures of high-speed jets. Good agreement was found by comparing the proposed similarity spectrum and a large set of combustion noise spectra. However, he argued that the proposal cannot be proven on a physical basis due to the lack of knowledge about the mechanism by which combustion noise is generated. 

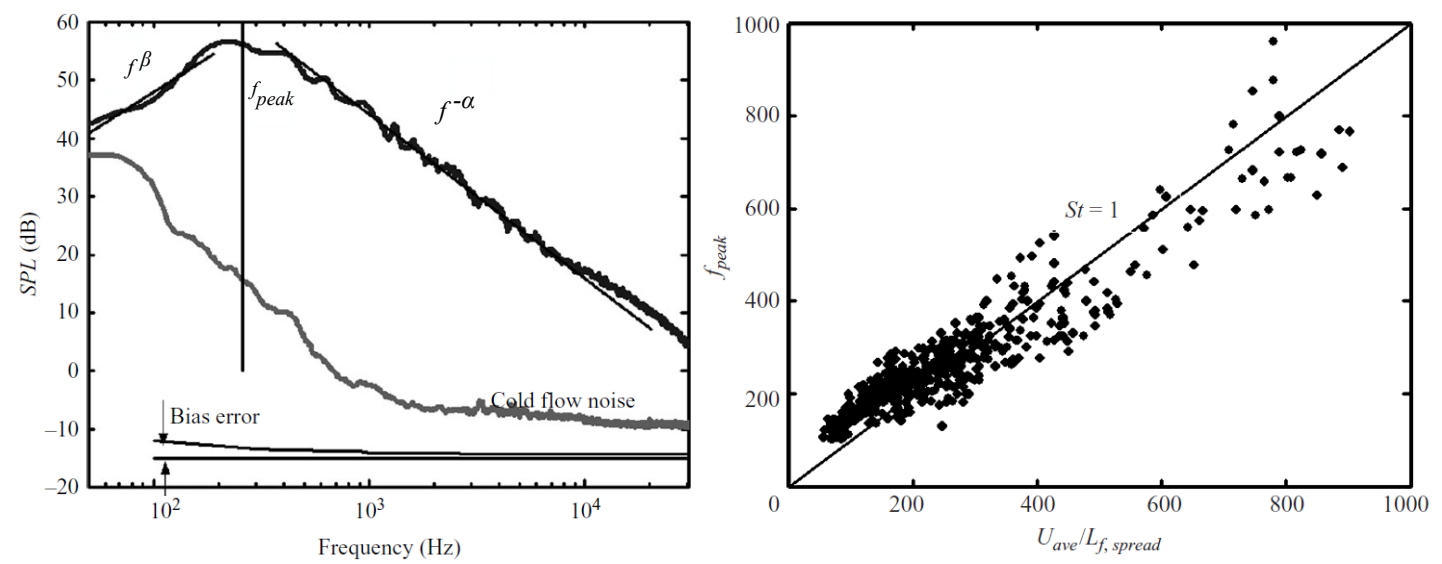

Figure 2.4: Typical spectral dependence of acetylene fuelled combustion noise (left), Strouhal number dependence of frequency of peak emission (right) Rajaram \& Lieuwen, 2009].

\subsection{Numerical Studies of Sound Generation by Premixed Flames}

Computational aeroacoustics (CAA) is referred to numerical methods which are associated with calculation of the flow-generated sound. Three different approaches can be used in the CAA to calculate the radiated sound to the far field. In the first approach which is the least expensive method, the computational domain only encompasses the source region, and the acoustic field is resolved using an acoustic analogy based on the near-field data obtained by the computation (Colonius et al. [1997]). In the second approach the information on a surface surrounding the source region, known as Kirchhoff surface, will be used to solve for the far field using linearised Euler equations or a wave equation (e.g. Bodony \& Lele [2005]). The last approach, which is the most expensive computationally, is called direct noise computation where both the source region and the acoustic field are resolved using the same governing equations.

While there has been considerable effort in the simulation of turbulent combustion and combustion instabilities over the past fifty years, there are few investigations devoted to the numerical analysis of sound generation by combusting flows. Most of the earlier studies of combustion noise, whether theoretical or ex- 
perimental, have tried to provide scaling laws. However, with the advent of high performance computing, CAA is becoming a more commonly used tool to examine sound generation by fluid flows. Advanced computational techniques such as Large Eddy Simulation (LES) and Direct Numerical Simulation (DNS) are now used in CAA, allowing better insight into the mechanisms of sound generation by combusting flows.

\subsubsection{LES Studies}

Large Eddy Simulation (LES) explicitly calculates the large turbulent scales whereas the smaller structures are modelled. The large scales, containing most of the turbulent energy, are resolved using so-called 'filtered' forms of the NavierStokes equations (Poinsot \& Veynante 2005]).

There are several numerical studies on sound generation by turbulent flames using LES. The focus of these studies have mainly placed on diffusion flames (e.g. Bui et al. 2005]; Birbaud et al. [2006]; Flemming et al. [2007]; Bui et al. [2008]; Bui et al. 2009]; Ihme et al. 2009]; Ihme \& Pitsch 2012]; Zhang et al. 2013]), with few studies dedicated to premixed flames (Silva et al. 2013] and Schlimpert et al. [2017]).

Although the significance of the heat release rate fluctuations was confirmed by these studies, varying levels of agreement between LES and experiment was observed Bui et al., 2009; Flemming et al., 2007 and several sources of uncertainty such as sub-grid modelling Ihme et al., 2009 and grid resolution Ihme et al., 2009. were found.

Out of a few studies on premixed flames, Silva et al. 2013] highlighted the importance of grid resolution in LES in terms of the prediction of heat release rate fluctuations and consequently the produced sound. In another study, Schlimpert et al. 2017] performed LES of noise generation by a premixed slot jet flame using a hybrid approach. In their simulation, the level-set approach was used to describe the motion of the flame with the so-called G-equation. One of the main findings of this study was that the heat release rate fluctuations can be used to predict the trend in the spectrum for the low to medium frequency part. However, in the high frequency region, the predicted trend of the spectrum becomes a 
strong function of the modelling parameter, namely, the Markstein length. This highlights the significance of modelling approaches for the prediction of sound in LES. Nonetheless, LES appears to be the only plausible tool for examining sound generation in practical turbulent flames.

\subsubsection{DNS Studies}

In contrast to LES, direct numerical simulation (DNS) should not suffer from problems associated with grid resolution or sub-grid modelling. DNS resolves all spatio-temporal scales of turbulence and chemistry. As a result, DNS is computationally very expensive, imposing severe limitation on using this method in practical applications which feature complex geometries and high Reynolds numbers. However, DNS can provide detailed information of the flow field which makes it a useful tool for understanding the involved physical processes as well as model development. DNS of sound generation by combusting flows is quite rare because of the computational cost incurred by resolving both the flame and the radiated sound simultaneously, and the sensitivity of the acoustic field to numerical issues that are particularly challenging in combusting flows Talei et al. 2014; Zhao \& Frankel, 2001].

Zhao \& Frankel 2001 simulated sound generation by an axisymmetric premixed, unconfined flame jet using DNS. They found that the heat release term affects not only the sound radiation level and its directionality, but also the structure of vortices in the flow field comparing with a non-reacting jet at similar conditions. In addition, a broader noise spectrum was observed as a result of combustion.

Talei et al. 2011 examined sound generation by planar, axisymmetric and spherically symmetric flame annihilation events. Their results showed that the amplitude of the far-field sound produced by the annihilation events depends on the flame thickness, particularly for the axisymmetric and spherically symmetric flame annihilation events, as showed in figure 2.5. They also observed significant acceleration in the flame speed prior to flame annihilation, consistent with the earlier reported experimental and numerical studies (e.g. Echekki et al. [1996]; Sun \& Law 1998). 
(a)

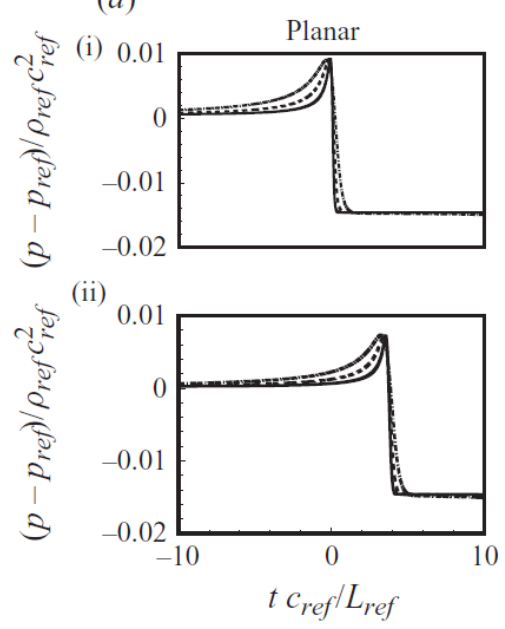

(b)
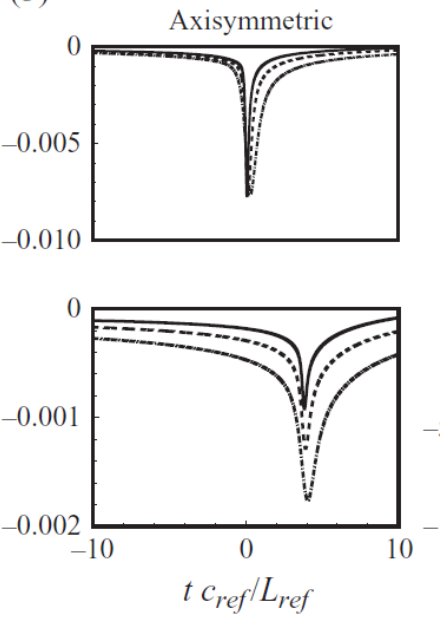

(c)
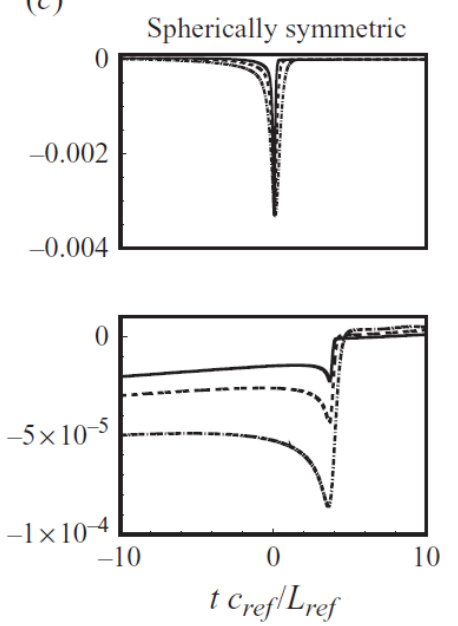

Figure 2.5: Non-dimensional pressure $\left(p-p_{\text {ref }}\right) / \rho_{\text {ref }} c_{\text {ref }}^{2}$ for flame annihilation from DNS (i) at the symmetry axis and (ii) in the far-field for different flame thicknesses, $\delta / L_{r e f}=0.1$ (solid line), $\delta / L_{r e f}=0.2$ (dashed), $\delta / L_{r e f}=0.4$ (dashdotted). Talei et al., 2011.

Talei et al. 2011 also proposed a theory based on Dowling's 1992 extended form of Lighthill's equation to relate the far-field sound to the flame key parameters. Apart from considering only the temporal heat release source term in Lighthill's acoustic analogy, additional assumptions of an infinitely thin flame and constant flame speed were also used. For planar annihilation, their theory resulted in good predictions of the overall pressure change; however, these assumptions led to significant under-prediction of the pressure amplitude in the far field for the axisymmetric and spherically symmetric annihilation events, as shown in figure 2.6. In a modified form of their theory, Talei et al. 2012a achieved an improved result by modelling the reaction rate as a $\delta$-function, considering a constant consumption speed and using the prorogation speed obtained from DNS, as shown in figure 2.7. They also showed that calculating the prorogation speed using the Markstein theory underestimates the amplitude of the produced sound (see figure $2.7 \mathrm{ii}$.

In later studies, Talei et al. 2014, 2012b] examined the response of twodimensional laminar flames to acoustic forcing. It was shown that for a range of 

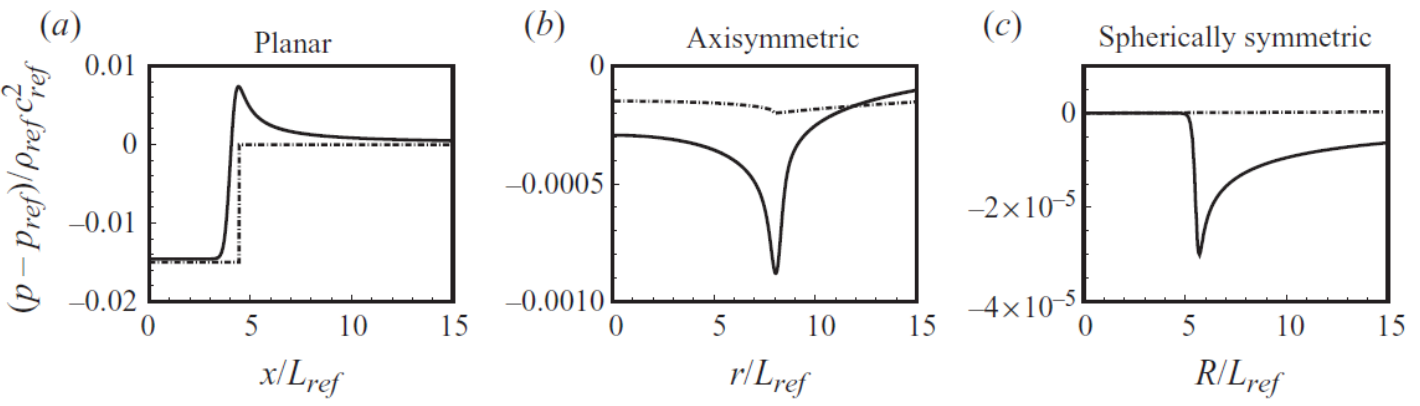

Figure 2.6: Non-dimensional pressure $\left(p-p_{\text {ref }}\right) / \rho_{\text {ref }} c_{\text {ref }}^{2}$ for the planar, axisymmetric and spherically symmetric flame annihilation from DNS (solid line), zero flame thickness (dash-dotted). TTalei et al., 2011]

forcing frequencies, two types of flame annihilation events can be observed. The first type was called 'flame pinch-off', where collision between the two curved flame fronts occurred and sound was generated. This annihilation event also created a pocket of unburned mixture which was subsequently consumed and led to the second type of annihilation event, referred to as 'island burn-out'. They also showed the importance of Lewis number in the flame-generated noise, with strong monopolar sound sources observed for $L e=1$ and 2 , while almost no sound for $L e=0.5$, as depicted in figure 2.8 .

Jiménez et al. 2015] performed a DNS study of sound generation by hydrogenair premixed laminar flame annihilation with full and simple chemistry. They examined planar annihilation from lean $(\phi=0.5)$ to rich $(\phi=3)$ conditions, corresponding to (effective) Lewis numbers ranging from 0.5 to 2.0, respectively. In their simple chemistry, the laminar flame speed matched that of the detailed chemistry simulation at the same equivalence ratio. They confirmed the significant role of the Lewis number in the sound generation process, as their previous, simple chemistry studies had found Talei et al. 2012a. b]. They also showed that the use of simple chemistry was reasonable in some cases where the effective Lewis number was equal or less than unity. Such conditions may also be achieved at premixed natural gas/air flames as well as premixed lean and stoichiometric hydrogen/air flames Law, 2006]. 

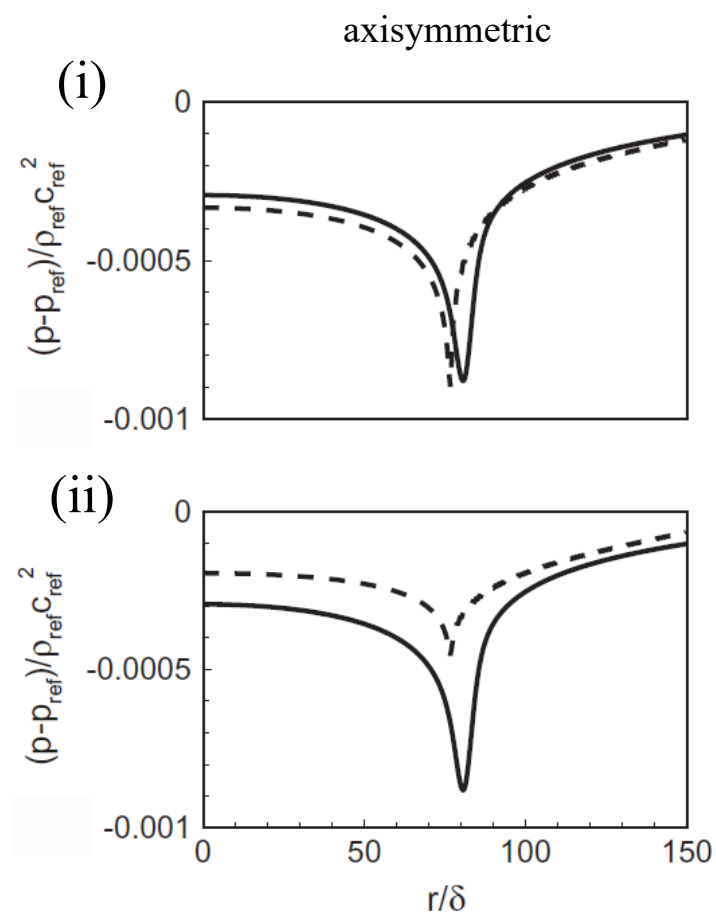

spherically symmetric
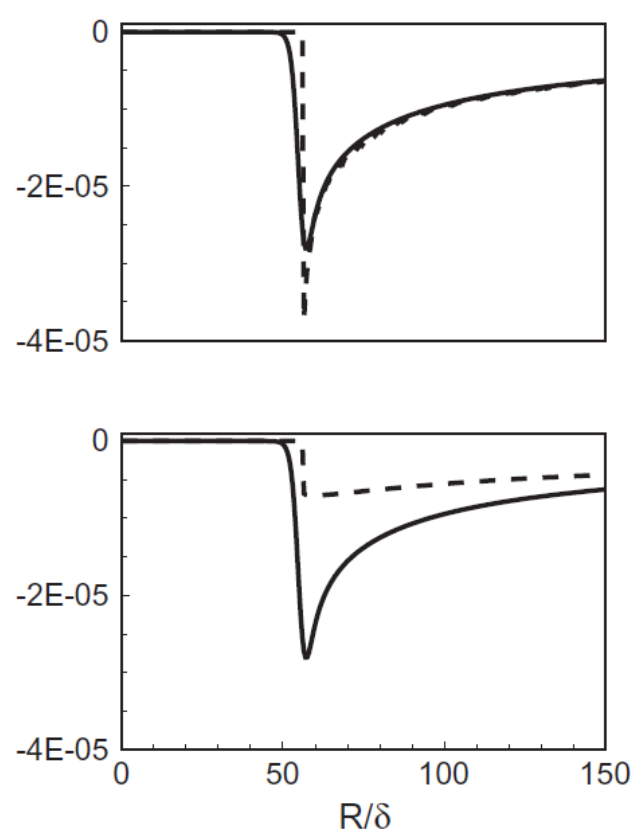

Figure 2.7: Comparison of DNS (solid) and solution of Lighthill's equation (dashed) at an instant after annihilation (i) obtaining propagation velocity from the simulation results and (ii) using the Markstein length theory. Talei et al., $2012 a$ 
(a)

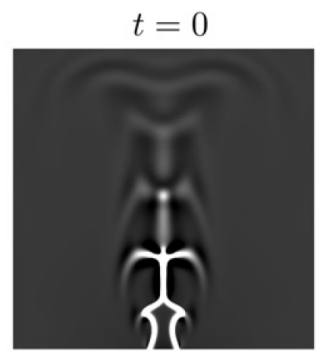

$t=T / 4$
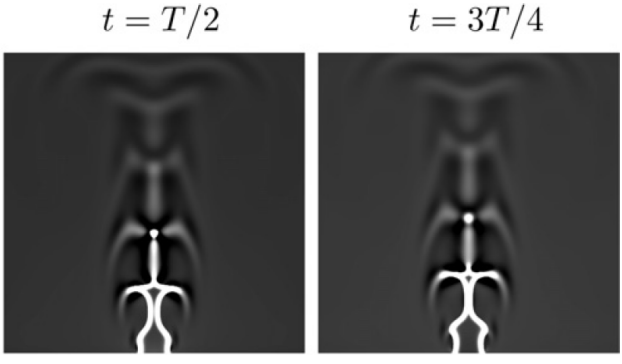

(b)
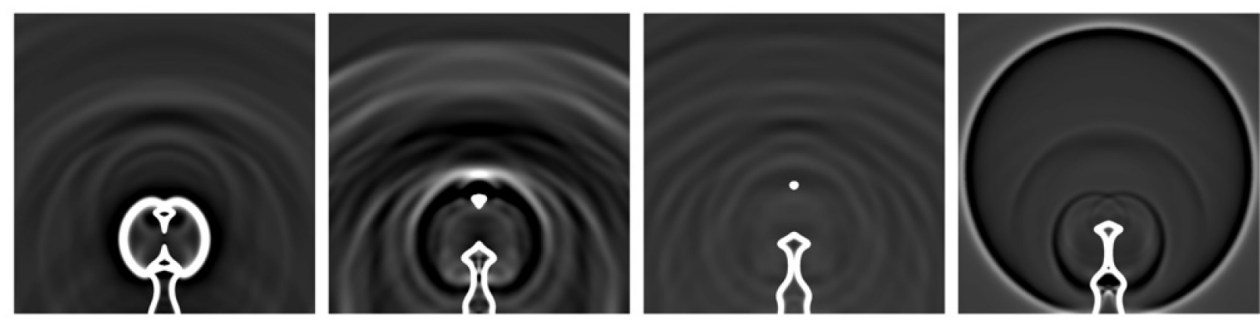

(c)

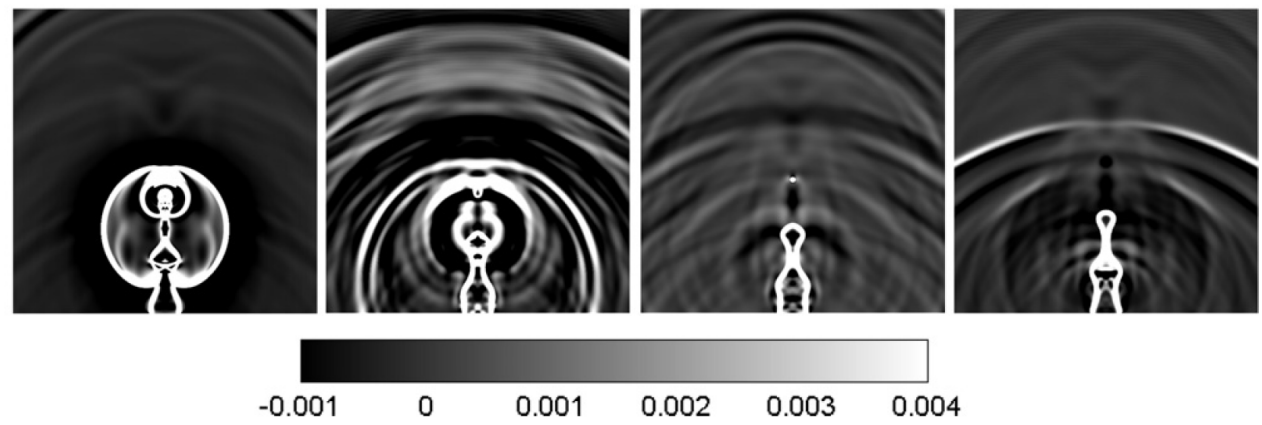

Figure 2.8: Dimensionless dilatation field for forced, laminar, 2D flames for $S t_{D}$ $=0.025$ and (a) $L e=0.5$, (b) $L e=1$ and (c) $L e=2$. (Talei et al. $[2012 b]$ ). 


\section{$2.7 \quad$ Summary}

This chapter provided a review of the literature on sound production by combusting flows with a particular interest on premixed flames. This commenced with a discussion of the fundamentals of combustion noise and Lighthill's acoustic analogy, followed by a review of the major experimental and numerical works that have appeared over several decades.

In one of the earlier studies of combustion noise, Smith \& Kilham 1963 showed that the emitted sound by open turbulent premixed flames can be modelled by a distribution of monopolar sources throughout the flame. Changes in the flame volume or equivalently the heat release rate fluctuations have also been demonstrated to be the primary source of sound in other studies of turbulent flames (e.g., Hurle et al. 1968]; Strahle 1978]; Kotake \& Takamoto 1990]; Dowling 1992]; Schuller et al. [2003]; Candel et al. 2004]; Swaminathan et al. 2011a]; Swaminathan et al. 2011b; Rajaram \& Lieuwen 2009]; Liu 2015]). However, there is relatively little known about the phenomena that produce large fluctuations of the heat release rate and therefore generated sound.

One mechanism that may result in significant changes in the heat release rate is premixed flame annihilation. Flame annihilation events can occur when two flame surfaces collide, leading to a rapid change in the consumption rate of the unburned gas and therefore large changes of the heat release rate. There are a few experimental studies which investigated this mechanism of sound generation (Kidin et al. [1988]; Schuller et al. 2003]; Candel et al. [2004]), highlighting the importance of these events. Numerical studies such as those performed by Talei et al. [2011], Talei et al. [2012a b], Brear et al. [2012], Talei et al. 2014] and Jiménez et al. 2015]) have also confirmed the observations in the experimental studies and provided additional insight into the key parameters affecting the strengths of these events. Nevertheless, the significance of annihilation events in noise generation by turbulent premixed flames appears to have received no examination, even though they have been observed in numerous non-acoustic studies of turbulent, premixed flames (e.g., Echekki et al. [1996]; Balachandran et al. 2005]; Griffiths et al. 2015])).

Recent advancements in high performance computing now allow us to use Di- 
rect Numerical Simulation (DNS) to examine computationally intensive problems such as sound generation by turbulent premixed flames. Such DNS study enables us to directly relate the sound produced to its sources, providing insight into the sound generation mechanism in unprecedented detail.

The aim of this thesis is therefore to study sound generation by turbulent premixed flames using DNS. In particular, special attention will be paid to the role of annihilation events in the sound generation process. 


\section{Chapter 3}

\section{Numerical Methods and DNS Setup}

\subsection{Introduction}

This chapter presents the numerical methods and the flow/flame configuration which are used to study noise generation by turbulent, premixed jet flames. Direct Numerical Simulation (DNS) is used in this study. DNS of sound generation by combusting flows is a difficult problem because of the computational cost incurred by resolving both the flame and the radiated sound simultaneously, and the sensitivity of the acoustic field to numerical noise which might be present in the solution of the governing equations. In this chapter, an introduction of the governing equations of reacting flows with single-step, irreversible chemistry will be presented first. This will be followed by the details of the boundary conditions used. After that, there will be a brief description of the DNS solver. Finally, the computational domain and the DNS setup will be discussed in detail.

\subsection{Governing Equations}

Assuming a single-step, irreversible chemical reaction, the dimensional governing equations for the conservation of mass, momentum, total energy, and reactant 
species mass fraction are, respectively, given as follows:

$$
\begin{gathered}
\frac{\partial \rho^{*}}{\partial t^{*}}+\frac{\partial \rho^{*} \mathbf{u}_{i}^{*}}{\partial \mathbf{x}_{i}^{*}}=0 \\
\frac{\partial \rho^{*} \mathbf{u}_{j}^{*}}{\partial t^{*}}+\frac{\partial \rho^{*} \mathbf{u}_{i}^{*} \mathbf{u}_{j}^{*}}{\partial \mathbf{x}_{i}^{*}}=-\frac{\partial p^{*}}{\partial \mathbf{x}_{j}^{*}}+\frac{\partial \tau_{i j}^{*}}{\partial \mathbf{x}_{i}^{*}}, \\
\frac{\partial \rho^{*} E_{t}^{*}}{\partial t^{*}}+\frac{\partial \mathbf{u}_{j}^{*}\left(\rho^{*} E_{t}^{*}+p^{*}\right)}{\partial \mathbf{x}_{j}^{*}}=\frac{\partial \mathbf{u}_{i}^{*} \tau_{i j}^{*}}{\partial \mathbf{x}_{j}^{*}}-\frac{\partial \mathbf{q}_{j}^{*}}{\partial \mathbf{x}_{j}^{*}},
\end{gathered}
$$

and

$$
\frac{\partial \rho^{*} Y_{R}^{*}}{\partial t^{*}}+\frac{\partial \rho^{*} \mathbf{u}_{j}^{*} Y_{R}^{*}}{\partial \mathbf{x}_{j}^{*}}=\frac{\partial}{\partial \mathbf{x}_{j}^{*}}\left(\rho^{*} D_{m} \frac{\partial Y_{R}^{*}}{\partial \mathbf{x}_{j}^{*}}\right)+\dot{\omega}_{R}^{*}
$$

where $x^{*}$ and $t^{*}$ are the spatial coordinate and time, respectively. In addition, $\rho^{*}$ is the mixture density, $u_{j}^{*}$ is the $j$-componentof flow velocity, $Y_{R}^{*}$ is the reactant mass fraction (progress variable), $p^{*}$ is the pressure, $E_{t}^{*}$ is the total energy per unit mass (including sensible, kinetic, and chemical energy), $\mathbf{q}_{j}^{*}$ is the heat flux vector, and $\tau_{i j}^{*}$ is the $i j$ - component of the viscous stress tensor. The quantities $E_{t}^{*}, \mathbf{q}_{j}^{*}$ (assuming constant heat capacity), $\tau_{i j}^{*}$ and $p^{*}$ (based on the ideal gas law) are given by,

$$
\begin{gathered}
E_{t}^{*}=\frac{p^{*}}{\rho^{*}(\gamma-1)}+\frac{1}{2} \mathbf{u}_{i}^{*} \mathbf{u}_{i}^{*}+Y_{R}^{*} Q^{*}, \\
\mathbf{q}_{j}^{*}=-\lambda \frac{\partial T^{*}}{\partial \mathbf{x}_{j}^{*}}-\sum_{k=1}^{N_{s}}\left(h_{k}^{0 *} \rho^{*} D_{m} \frac{\partial Y_{k}^{*}}{\partial \mathbf{x}_{j}^{*}}\right), \\
\tau_{i j}^{*}=\mu^{*}\left[\frac{\partial \mathbf{u}_{i}^{*}}{\partial \mathbf{x}_{j}^{*}}+\frac{\partial \mathbf{u}_{j}^{*}}{\partial \mathbf{x}_{i}^{*}}-\frac{2}{3} \frac{\partial \mathbf{u}_{k}^{*}}{\partial \mathbf{x}_{k}^{*}} \delta_{i j}\right]
\end{gathered}
$$




$$
p^{*}=\rho^{*}\left(\frac{R}{M}\right) T^{*}
$$

where $\gamma$ is the heat capacity ratio, $Q^{*}$ is the heat of reaction per unit mass of reactant, $h_{k}^{0 *}$ is the chemical enthalpy of formation of species $k$ evaluated at reference temperature $T_{r e f}, \mu^{*}$ is the dynamic viscosity, $D_{m}$ is the binary mass diffusion coefficient, $\lambda$ is the mixture thermal conductivity, $R$ is the ideal gas constant, $M_{k}$ is the species molecular weight, $M$ is the mixture molecular weight, and $\delta_{i j}$ is the Kronecker delta. The variation of $\mu^{*}$ with temperature is modelled using a power-law Poinsot et al., 1992,

$$
\mu^{*}=\mu_{\text {ref }}\left(\frac{T^{*}}{T_{r e f}}\right)^{0.76}
$$

where $\mu_{r e f}^{*}$ is the fresh gas absolute viscosity. The reactant species reaction rate $\dot{\omega}_{R}^{*}$ may be obtained using the Arrhenius law,

$$
\dot{\omega}_{F}^{*}=\Lambda \rho^{*} Y_{F}^{*} \exp \left[-\frac{\beta(1-\theta)}{1-\alpha(1-\theta)}\right]
$$

where

$$
\Lambda=B \exp \left(-\frac{\beta}{\alpha}\right), \theta=\frac{T^{*}-T_{u}^{*}}{T_{f}^{*}-T_{u}^{*}}, \alpha=\frac{T_{f}^{*}-T_{u}^{*}}{T_{f}^{*}} \text { and } \beta=\frac{E_{a}^{*}\left(T_{f}^{*}-T_{u}^{*}\right)}{R T_{f}^{2 *}} .
$$

Note that $B$ is the pre-exponential factor, $\beta$ is the Zel'dovich number, $T_{f}^{*}$ is the adiabatic flame temperature, $T_{u}^{*}$ is the fresh (unburned) mixture temperature, $E_{a}^{*}$ is the activation energy and $\alpha$ is the heat release parameter.

\section{Non-dimensional Equations}

The non-dimensional form of the governing equations are solved in this study. The non-dimensional variables may be expressed as,

$$
\mathbf{x}_{j}=\frac{\mathbf{x}_{j}^{*}}{D}, t=\frac{t^{*} c_{r e f}}{D}, \mathbf{u}_{j}=\frac{\mathbf{u}_{j}^{*}}{c_{r e f}}, \rho=\frac{\rho^{*}}{\rho_{r e f}}, T=\frac{T^{*}}{(\gamma-1) T_{r e f}},
$$




$$
Y_{R}=\frac{Y_{R}^{*}}{Y_{R, r e f}}, p=\frac{p^{*}}{\rho_{r e f} c_{r e f}^{2}}, E_{t}=\frac{E_{t}^{*}}{c_{r e f}^{2}} \text { and } \mu=\frac{\mu^{*}}{\mu_{r e f}} .
$$

The subscript ref refers to the reference variables and corresponds to the values of the reactant at the jet inlet boundary. The following non-dimensional numbers are also defined:

$$
\begin{gathered}
R e=\left(\frac{c D}{\nu}\right)_{r e f}, \operatorname{Pr}=\left(\frac{\nu}{D_{t h}}\right)_{r e f}, L e=\left(\frac{D_{t h}}{D_{m}}\right)_{r e f}, \\
D a=\left(\frac{\Lambda D}{c}\right)_{r e f} \text { and } S c=L e \operatorname{Pr} .
\end{gathered}
$$

Note that $c$ is the sonic velocity, $D$ is the jet diameter, $R e$ is the acoustic Reynolds number, $\operatorname{Pr}$ is the Prandtl number, $S c$ is the Schmidt number, Le is the Lewis number, $D a$ is the Damköhler number, $D_{t h}$ is the thermal diffusivity coefficient, and $\nu$ is the kinematic viscosity. Using these parameters, the non-dimensional form of the governing equations may be expressed as

$$
\begin{gathered}
\frac{\partial \rho}{\partial t}+\frac{\partial \rho \mathbf{u}_{i}}{\partial \mathbf{x}_{i}}=0 \\
\frac{\partial \rho \mathbf{u}_{j}}{\partial t}+\frac{\partial \rho \mathbf{u}_{i} \mathbf{u}_{j}}{\partial \mathbf{x}_{i}}=-\frac{\partial p}{\partial \mathbf{x}_{j}}+\frac{\partial \tau_{i j}}{\partial \mathbf{x}_{i}}, \\
\frac{\partial \rho E_{t}}{\partial t}+\frac{\partial \mathbf{u}_{j}\left(\rho E_{t}+p\right)}{\partial \mathbf{x}_{j}}=\frac{\partial \mathbf{u}_{i} \tau_{i j}}{\partial \mathbf{x}_{j}}-\frac{\partial \mathbf{q}_{j}}{\partial \mathbf{x}_{j}}, \text { and } \\
\frac{\partial \rho Y_{R}}{\partial t}+\frac{\partial \rho \mathbf{u}_{j} Y_{R}}{\partial \mathbf{x}_{j}}=\frac{\partial}{\partial \mathbf{x}_{j}}\left(\frac{1}{R e S c} \mu \frac{\partial Y_{R}}{\partial \mathbf{x}_{j}}\right)+\dot{\omega}_{R}
\end{gathered}
$$

where

$$
p=\frac{\gamma-1}{\gamma} \rho T
$$




$$
\begin{gathered}
E_{t}=\frac{T}{\gamma}+\frac{1}{2} \mathbf{u}_{i} \mathbf{u}_{i}+\frac{\tau}{\gamma-1} Y_{R}, \\
\tau_{i j}=\frac{\mu}{R e}\left[\frac{\partial \mathbf{u}_{i}}{\partial \mathbf{x}_{j}}+\frac{\partial \mathbf{u}_{j}}{\partial \mathbf{x}_{i}}-\frac{2}{3} \frac{\partial \mathbf{u}_{k}}{\partial \mathbf{x}_{k}} \delta_{i j}\right] \\
\mathbf{q}_{j}=-\frac{\mu}{\operatorname{Re} \operatorname{Pr}} \frac{\partial T}{\partial \mathbf{x}_{j}}-\frac{\mu}{\operatorname{Re} S c} \frac{\tau}{(\gamma-1)} \frac{\partial Y_{R}}{\partial \mathbf{x}_{j}}
\end{gathered}
$$

and

$$
\dot{\omega}_{R}=-D a \rho Y_{R} \exp \left[-\frac{\beta(1-\theta)}{1-\alpha(1-\theta)}\right]
$$

with

$$
\tau=\alpha /(1-\alpha), \text { and } \theta=\frac{(\gamma-1) T-1}{\tau}
$$

\subsection{Boundary Conditions}

Using high-order accurate computational methods enable us to have low levels of numerical dissipation and dispersion errors, but the sensitivity of such methods to the boundary treatment is significant (Yoo et al. [2005]). The implementation of boundary conditions in the simulation of compressible flows can be based on characteristic wave analysis. Poinsot \& Lele 1992 put forward a boundary treatment for reacting flows known as Navier-Stokes Characteristic Boundary Conditions (NSCBC), which was an extension to the earlier work of Thompson [1990] on boundary conditions for a system of hyperbolic equations. Poinsot \& Lele 1992 rearranged the Navier-Stokes equations such that the characteristic waves propagating in the direction normal to the boundary can be treated properly. In NSCBC, outgoing characteristic waves from the computational domain are computed using knowledge from inside of the domain. The incoming characteristic waves are then treated depending on the type of the boundary condition.

The objective of the NSCBC technique is to suppress spurious acoustic wave 
reflections into the computational domain at open boundaries while retaining proper communication between the interior and exterior of the domain. Poinsot \& Lele's [1992] approach in evaluating the wave amplitude variations is based upon a local one-dimensional inviscid (LODI) problem neglecting transverse, viscous, and reaction terms. Therefore, some researchers have attempted to include the effect of chemical reaction (Sutherland \& Kennedy [2003]), multi-dimensionality (Yoo et al. 2005, Yoo \& Im 2007]), and proper treatment of edges and corners (Lodato et al. 2008]).

In this study, the formulation proposed by Yoo \& Im 2007 has been used. The reason for this choice will be discussed in more details in section 3.5.5. The compressible Navier-Stokes, total energy and species conservation equations for reacting flows with a single-step, irreversible reaction mechanism can be transformed into a characteristic form in terms of primitive variables as follows:

$$
\frac{\partial}{\partial t}\left[\begin{array}{c}
u \\
v \\
w \\
\rho \\
p \\
Y_{R}
\end{array}\right]+\left[\begin{array}{c}
\left(L_{5}^{(x)}-L_{1}^{(x)}\right) / \rho c \\
L_{3}^{(x)} \\
L_{4}^{(x)} \\
L_{2}^{(x)}+\left(\begin{array}{c}
\left(L_{5}^{(x)}+L_{1}^{(x)}\right) / c^{2} \\
L_{5}^{(x)}+L_{1}^{(x)} \\
L_{6}^{(x)}
\end{array}\right]+\left[\begin{array}{c}
\mathbf{v}_{t} \cdot \nabla_{t} u \\
\mathbf{v}_{t} \cdot \nabla_{t} v+(1 / \rho) \partial p / \partial y \\
\mathbf{v}_{t} \cdot \nabla_{t} w+(1 / \rho) \partial p / \partial z \\
\mathbf{v}_{t} \cdot \nabla_{t} \rho+\rho \nabla_{t} \cdot \mathbf{v}_{t} \\
\mathbf{v}_{t} \cdot \nabla_{t} p+\gamma p \nabla_{t} \cdot \mathbf{v}_{t} \\
\mathbf{v}_{t} \cdot \nabla_{t} Y_{R}
\end{array}\right] \\
=\left[\begin{array}{c}
d_{u} \\
d_{v} \\
d_{w} \\
d_{\rho} \\
d_{p} \\
d_{Y_{R}}
\end{array}\right]+\left[\begin{array}{c}
s_{u} \\
s_{v} \\
s_{w} \\
s_{\rho} \\
s_{p} \\
s_{Y_{R}}
\end{array}\right]
\end{array}\right.
$$

where $L_{k}^{(x)}$ (for $1 \leq k \leq 6$ ) are the amplitudes of different characteristic waves propagating in the $x$-direction, 


$$
\mathbf{L}^{(x)}=\left[\begin{array}{c}
L_{1}^{(x)} \\
L_{2}^{(x)} \\
L_{3}^{(x)} \\
L_{4}^{(x)} \\
L_{5}^{(x)} \\
L_{6}^{(x)}
\end{array}\right]=\left[\begin{array}{c}
\lambda_{1}^{(x)} \cdot \frac{1}{2}\left(\frac{\partial p}{\partial x}-\rho c \frac{\partial u}{\partial x}\right) \\
\lambda_{2}^{(x)}\left(\frac{\partial \rho}{\partial x}-\frac{1}{c^{2}} \frac{\partial p}{\partial x}\right) \\
\lambda_{3}^{(x)} \frac{\partial v}{\partial x} \\
\lambda_{4}^{(x)} \frac{\partial w}{\partial x} \\
\lambda_{5}^{(x)} \cdot \frac{1}{2}\left(\frac{\partial p}{\partial x}+\rho c \frac{\partial u}{\partial x}\right) \\
\lambda_{6}^{(x)} \frac{\partial Y_{R}}{\partial x}
\end{array}\right]
$$

The characteristic velocities $\lambda_{k}^{(x)}$ are then given by,

$$
\lambda_{1}^{(x)}=u-c, \lambda_{2}^{(x)}=\lambda_{3}^{(x)}=\lambda_{4}^{(x)}=\lambda_{6}^{(x)}=u, \lambda_{5}^{(x)}=u+c .
$$

Note that in equation $3.24, \mathbf{v}$ is the velocity vector, and the subscript $t$ represents tangential $(y$ and $z$ ) directions. In addition, the viscous terms and source terms in equation 3.24 are, respectively, given by,

$$
\left[\begin{array}{c}
d_{u} \\
d_{v} \\
d_{w} \\
d_{\rho} \\
d_{p} \\
d_{Y_{R}}
\end{array}\right]=\left[\begin{array}{c}
(1 / \rho) \nabla_{j} \cdot \tau_{j x} \\
(1 / \rho) \nabla_{j} \cdot \tau_{j y} \\
(1 / \rho) \nabla_{j} \cdot \tau_{j z} \\
0 \\
(\gamma-1)\left[\tau_{j k}: \nabla_{j} u_{k}-\nabla_{j} \cdot q_{j}\right] \\
(1 / \rho) \nabla_{j} \cdot\left(\rho D_{m} \nabla_{j} Y_{R}\right)
\end{array}\right] \text { and }\left[\begin{array}{c}
s_{u} \\
s_{v} \\
s_{w} \\
s_{\rho} \\
s_{p} \\
s_{Y_{R}}
\end{array}\right]=\left[\begin{array}{c}
0 \\
0 \\
0 \\
0 \\
(1-\gamma)\left(\dot{\omega}_{R} Q\right) \\
\frac{1}{\rho} \dot{\omega}_{R}
\end{array}\right] \text {. }
$$

Note that the similar form of equations is obtained in the $y$ and $z$ directions.

In order to implement the non-reflecting boundary conditions, the wave amplitudes $L_{k}^{(x)}$ are determined depending on the type of boundary conditions. Equations 3.24 will then be solved using the modified $L_{k}^{(x)}$ at the boundaries, along with the original governing equations inside the domain. The incoming or outgoing nature of each wave can be understood from the sign of the characteristic velocities $\lambda_{k}^{(x)}$. The outgoing waves do not need to be modified because they do not cause any reflection. However, some additional modelling and constraints 
for unknown incoming waves are required to prevent spurious reflections. The wave amplitudes of the incoming and outgoing waves in the $x$-direction may be expressed as follows (Yoo et al. 2005 and Yoo \& Im 2007]):

- For subsonic non-reflecting inflow boundary at $x=0$ :

$$
\begin{cases}u-c<0 & L_{1}^{(x)} \quad \text { computed } \\ u>0 & L_{2}^{(x)}=\beta_{2}\left(T-T_{\text {target }}\right)+\Im_{2}^{(x)}+V_{2}^{(x)}+S_{2}^{(x)} \\ u>0 & L_{3}^{(x)}=\beta_{3}\left(v-v_{\text {target }}\right)+\Im_{3}^{(x)}+V_{3}^{(x)}+S_{3}^{(x)} \\ u>0 & L_{4}^{(x)}=\beta_{4}\left(w-w_{\text {target }}\right)+\Im_{4}^{(x)}+V_{4}^{(x)}+S_{4}^{(x)} \\ u+c>0 & L_{5}^{(x)}=\beta_{5}\left(u-u_{\text {target }}\right)+\Im_{5}^{(x)}+V_{5}^{(x)}+S_{5}^{(x)} \\ u>0 & L_{6}^{(x)}=\beta_{6}\left(Y_{R}-Y_{R, \text { target }}\right)+\Im_{6}^{(x)}+V_{6}^{(x)}+S_{6}^{(x)}\end{cases}
$$

- For subsonic non-reflecting outflow boundary at $x=L_{x}$ :

$$
\begin{cases}u-c<0 & L_{1}^{(x)}=\alpha_{1}\left(p-p_{\text {target }}\right)-\left(1-\alpha_{1}\right) \Im_{1}^{(x)}+V_{1}^{(x)}+S_{1}^{(x)} \\ u>0 & L_{2}^{(x)} \text { computed } \\ u>0 & L_{3}^{(x)} \text { computed } \\ u>0 & L_{4}^{(x)} \text { computed } \\ u>0 & L_{6}^{(x)} \text { computed }\end{cases}
$$

Note that $\beta_{k}$ in equation 3.28 and $\alpha_{1}$ in equation 3.29 are relaxation factors allowing smooth transition to the target variables while suppressing spurious reflections from the boundary Lodato et al., 2008; Yoo \& Im, 2007; Yoo et al., 2005. Transverse $\left(\Im^{(x)}\right)$, viscous $\left(V^{(x)}\right)$ and source $\left(S^{(x)}\right)$ terms in the $x$-direction are, respectively, given by (subscript $t$ denotes the $y$ - and $z$-directions), 


$$
\begin{gathered}
\left\{\begin{array}{l}
\Im_{1}^{(x)}=-\left(\mathbf{v}_{t} \cdot \nabla_{t} p+\gamma p \nabla_{t} \cdot \mathbf{v}_{t}-\rho c \mathbf{v}_{t} \cdot \nabla_{t} u\right) / 2 \\
\Im_{2}^{(x)}=-\nabla_{t} \cdot\left(\rho \mathbf{v}_{t}\right)+\left(\mathbf{v}_{t} \cdot \nabla_{t} p+\gamma p \nabla_{t} \cdot \mathbf{v}_{t}\right) / c^{2} \\
\Im_{3}^{(x)}=-\left(\mathbf{v}_{t} \cdot \nabla_{t} v+(1 / \rho) \partial p / \partial y\right) \\
\Im_{4}^{(x)}=-\left(\mathbf{v}_{t} \cdot \nabla_{t} w+(1 / \rho) \partial p / \partial z\right) \\
\Im_{5}^{(x)}=-\left(\mathbf{v}_{t} \cdot \nabla_{t} p+\gamma p \nabla_{t} \cdot \mathbf{v}_{t}+\rho c \mathbf{v}_{t} \cdot \nabla_{t} u\right) / 2 \\
\Im_{6}^{(x)}=-\mathbf{v}_{t} \cdot \nabla_{t} Y_{R}
\end{array}\right. \\
\left\{\begin{array} { l } 
{ V _ { 1 } ^ { ( x ) } = ( d _ { p } - \rho c d _ { u } ) / 2 } \\
{ V _ { 2 } ^ { ( x ) } = d _ { \rho } - d _ { p } / c ^ { 2 } } \\
{ V _ { 3 } ^ { ( x ) } = d _ { v } } \\
{ V _ { 4 } ^ { ( x ) } = d _ { w } } \\
{ V _ { 5 } ^ { ( x ) } = ( d _ { p } + \rho c d _ { u } ) / 2 } \\
{ V _ { 6 } ^ { ( x ) } = d _ { Y _ { R } } }
\end{array} \quad \text { and } \left\{\begin{array}{l}
S_{1}^{(x)}=\left(s_{p}-\rho c s_{u}\right) / 2 \\
S_{2}^{(x)}=s_{\rho}-s_{p} / c^{2} \\
S_{3}^{(x)}=s_{v} \\
S_{4}^{(x)}=s_{w} \\
S_{5}^{(x)}=\left(s_{p}+\rho c s_{u}\right) / 2 \\
S_{6}^{(x)}=s_{Y_{R}}
\end{array}\right.\right.
\end{gathered}
$$

\subsection{Numerical Solver}

In this study, direct numerical simulations (DNS) of jet premixed flames with a single step chemistry model are carried out using a modified version of the numerical solver S3D Chen et al., 2009 known as S3D-SC Karami et al., 2015, $2016 a b$. This solver features an $8^{\text {th }}$ order central differencing scheme for spatial derivatives, combined with a 6 -stage, $4^{\text {th }}$ order explicit Runge-Kutta time integrator. The governing equations are solved on a conventional rectangular Cartesian mesh. Since S3D uses almost non-dissipative, non-dispersive numerical schemes, it can be reliably used in Computational Aero-Acoustics (CAA), where the high-order accuracy of the solver is vital. In addition, S3D is a massively parallel and MPI-compatible solver, making it suitable for simulating turbulent flames which are computationally very expensive. 


\subsection{Flow Configuration and Numerical Setup}

\subsubsection{Computational Domain and Initial Conditions}

As shown in figure 3.1, a subsonic round jet of premixed reactant is injected into an open environment of combustion products at the adiabatic flame temperature. Methane/air combustion is considered at two different equivalence ratios $(\phi=0.7$ and 1.0), with the reactants preheated to $T_{u}=800 \mathrm{~K}$ at $1 \mathrm{~atm}$. The choice of these equivalence ratios allows a parametric study on the effect of chemistry on the noise generated by turbulent premixed flames. Note that lean combustion with high levels of preheat is commonly used in modern gas turbines. A coflow with a low velocity magnitude (1\% of the jet mean velocity) surrounds the jet flame at the inflow at the product temperature.

The temperature and reactant species mass fraction profiles at the inlet boundary (see figure 3.2 ) were obtained using an unstrained laminar flame solution computed with the flame parameters as the turbulent cases summarised in table 3.1 .

The mean and the root mean square (RMS) profiles of the inlet streamwise velocity (see figure 3.2b) were adapted from the DNS study of Wu \& Moin 2008 for a fully developed turbulent pipe flow at $R e_{D}=5300$. It should be noted that the choice of Reynolds number and $\delta_{t h} / D$ were constrained by the computational cost. There is a difference in the inlet Mach numbers in order to keep the Reynolds number and the normalised laminar flame thickness $\left(\delta_{t h} / D=0.07\right)$ the same for both values of $\phi$, as can be seen in table 3.1 .

\subsubsection{Flame Parameters}

A simple chemistry model is used to reduce the computational cost, since the resolution required for the study of sound generation by turbulent, premixed flames is significantly higher than for non-acoustic studies of combustion Talei et al. 2014. Whilst single-step chemistry is not always adequate to describe the flame structure, it can nonetheless be used to match several important parameters such as the laminar flame speed, the flame thickness, the temperature ratio and the Lewis number. The use of simple chemistry is therefore a logical first step 


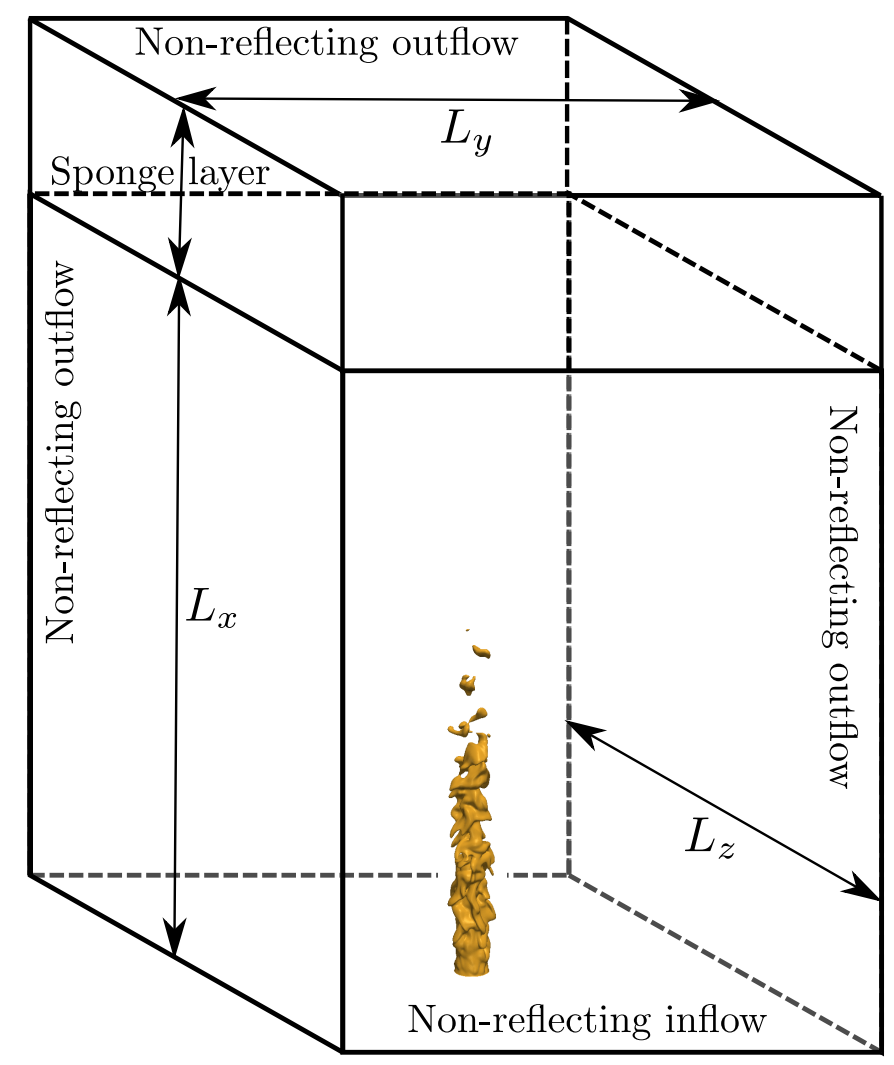

Figure 3.1: Schematic of the computational domain (not to scale). Note that the coordinate origin is located at the jet centreline on the inflow plane.

in such studies, and is reasonably common in the literature (e.g., Poinsot et al. [1992]; Haworth \& Poinsot [1992]; Chakraborty \& Cant [2009]; Dunstan et al. [2013]; Karami et al. [2015]; Chakraborty et al. [2016]).

The required flame parameters (see table 3.1) were obtained from PREMIX computations using GRI-MECH 3.0 Kee et al., 1985. The laminar flame thickness $\delta_{t h}$ was estimated as the thermal thickness Poinsot \& Veynante 2005]

$$
\delta_{t h}=\left(T_{b}-T_{u}\right) /\left|\frac{\mathrm{d} T}{\mathrm{~d} x}\right|_{\max }
$$

In order to determine the Zel'dovich number for each particular case, the activation temperature $(E / R)$ of the mixture was calculated based on the mass burning 
(a)

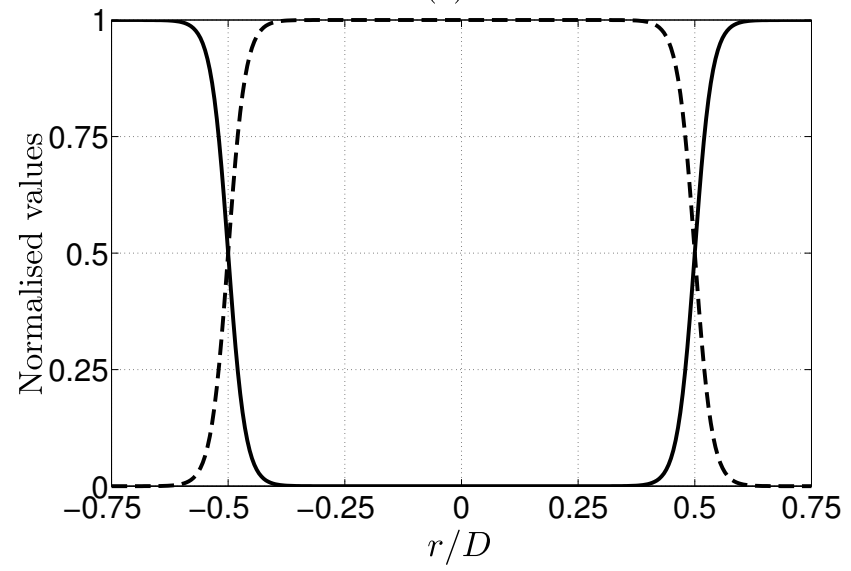

(b)

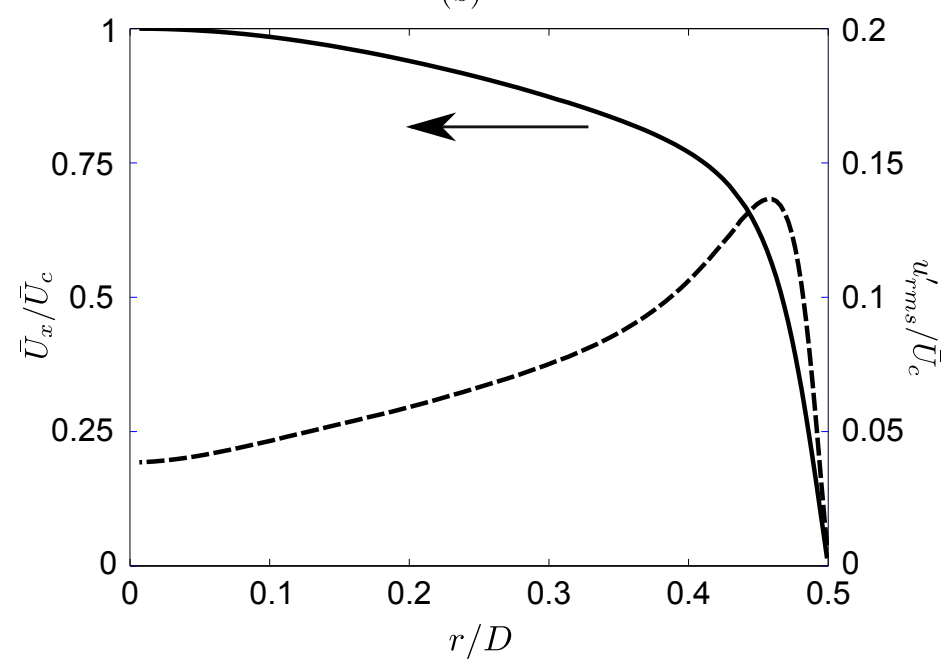

Figure 3.2: (a) Normalised temperature $\theta$ (solid) and reactant species mass fraction $\left(Y_{R}\right)$ (dashed) profiles at the inlet boundary; (b) The mean inlet streamwise velocity $\left(\bar{U}_{x} / \bar{U}_{c}\right)$ profile (solid) and the normalised RMS of streamwise velocity fluctuations $\left(u_{r m s}^{\prime} / \bar{U}_{c}\right)$ (dashed) at the inlet boundary.

flux $\left(f=\rho_{u} S_{L}\right)$ and adiabatic flame temperature as follows:

$$
\frac{E}{R}=-2 \frac{\mathrm{d} \ln (f)}{\mathrm{d}\left(1 / T_{b}\right)_{p, \phi}}
$$

The right hand side of equation 3.32 was evaluated from PREMIX computations by calculating mass burning flux and adiabatic flame temperature for the given pressure $p$ and equivalence ratio $\phi$, and by slightly varying its magnitude 


\begin{tabular}{lcc}
\hline \hline & $\phi=1.0$ & $\phi=0.7$ \\
Inlet jet diameter $(D)$ in mm & 3.94 & 4.63 \\
Domain size $\left(L_{x} \times L_{y} \times L_{z}\right)$ & $20 D \times 16 D \times 16 D$ \\
Grid resolution & $2412 \times 1040 \times 1040$ \\
Mean inlet jet Mach number & 0.35 & 0.26 \\
Co-flow Mach number & 0.0035 & 0.0026 \\
Heat release parameter $(\alpha)$ & 0.675 & 0.637 \\
Jet Reynolds number $\left(R e_{D}\right)$ & 5300 & 5300 \\
Inlet centreline turbulence intensity $\left(u_{r m s}^{\prime} / \overline{\mathrm{U}}_{j}\right)$ & 0.037 & 0.037 \\
Kolmogorov length scale $(\eta / D)$ & 0.014 & 0.014 \\
Longitudinal integral length scale $\left(l_{11} / D\right)$ & 0.30 & 0.30 \\
Transverse integral length scales $\left(l_{22,33} / D\right)$ & 0.15 & 0.15 \\
$\delta_{t h} / D$ & 0.07 & 0.07 \\
$S_{L} / c_{r e f}$ & 0.00422 & 0.00325 \\
$\beta$ & 7.9 & 5.5 \\
$D a$ & 8.6 & 3.2 \\
$P r$ & 0.72 & 0.72 \\
$L e$ & 1.0 & 1.0 \\
\hline \hline
\end{tabular}

Table 3.1: DNS parameters.

through the substitution of a small amount of the nitrogen by inert argon Law \& Sung, 2000. The (effective) Lewis number for each case was also computed using PREMIX and an equivalence-ratio-dependent expression proposed by Bechtold \& Matalon 2001] as follows:

$$
L e_{e f f}=1+\frac{\left(L e_{E}-1\right)+\left(L e_{D}-1\right) A}{1+A}
$$

with $A=1+\beta(\Phi-1)$. Note that the subscripts $E$ and $D$ denote the excess and deficient reactants, respectively, (in our study for $\phi=0.7 E=O_{2}$ and $D=\mathrm{CH}_{4}$; and for $\phi=1.0$ either of selection is correct). In addition, $\Phi$ is equal to the equivalence ratio $\phi$ for fuel-rich mixtures and its reciprocal $1 / \phi$ for fuellean mixtures. It should be stated that $L e_{e f f}$ is a weighted average of the Lewis numbers of the two reactants. Under exact stoichiometric conditions, $A=1$, both Lewis numbers are weighted evenly and $L e_{e f f}$ reduces to an arithmetic mean value. For non-stoichiometric mixtures, $A>1$, the Lewis number corresponding to the deficient reactant is weighted more heavily. 
Given the flame parameters obtained from PREMIX computations, a procedure is followed to achieve the target values of $S_{L}$ and $\delta_{t h}$ in S3D-SC. Figure 3.3 illustrates the flowchart for determining the correct magnitudes of $D a$ (Damköhler number) and $R e$ (acoustic Reynolds number) such that the values of $S_{L}$ and $\delta_{t h}$ calculated from a laminar premixed flame simulation using S3D-SC can be matched to those calculated from a PREMIX computation with GRI-MECH 3.0. The physical basis for this procedure is due to the fact that the flame speed is proportional to $\sqrt{\dot{\omega}_{R} \cdot D_{t h}}$ while the flame thickness is proportional to $\sqrt{D_{t h} / \dot{\omega}_{R}}$ Turns, 2000].

\subsubsection{Turbulence Feed Setup}

To impose the velocity fluctuations at the inlet, a homogeneous isotropic turbulence field based on a prescribed turbulent energy spectrum Passot \& Pouquet, 1987 with a turbulence intensity of $3.7 \%$ is first produced. This value is equal to the turbulent intensity of the pipe flow simulation at the centreline, reported by Wu \& Moin [2008]. The obtained velocity fluctuations were then rescaled using the turbulent intensity profile from $\mathrm{Wu} \&$ Moin 2008, shown in figure 3.2b. The resulting frozen velocity field was then added to the mean inlet velocity using Taylor's hypothesis.

Figure 3.4 depicts the inlet spectrum of streamwise turbulent kinetic energy $\left(u^{\prime 2}\right)$ computed at the jet centreline. As can be seen, the spectrum decreases with increasing frequency following the well-known $f^{-5 / 3}$ law for the kinetic energy spectrum in the inertial subrange [Peters, 2000].

\subsubsection{Grid Resolution}

The domain was resolved by a 3D, structured, Cartesian mesh. A uniform grid was used in the streamwise direction. An algebraically stretched mesh with an increasing grid spacing $\left(\left(\Delta_{i+1} / \Delta_{i}\right)-1\right)$ not exceeding $2 \%$ (c.f. figure 3.5) was used for $|y, z|>1.5 D$. The resulting mesh provides a uniform grid spacing with 200 grid points per jet diameter over a region covering one $D$ on each side of the jet. This ensures that the instantaneous flame brush always remains within this finely resolved region, with 14 grid points per laminar flame thickness. To suppress 


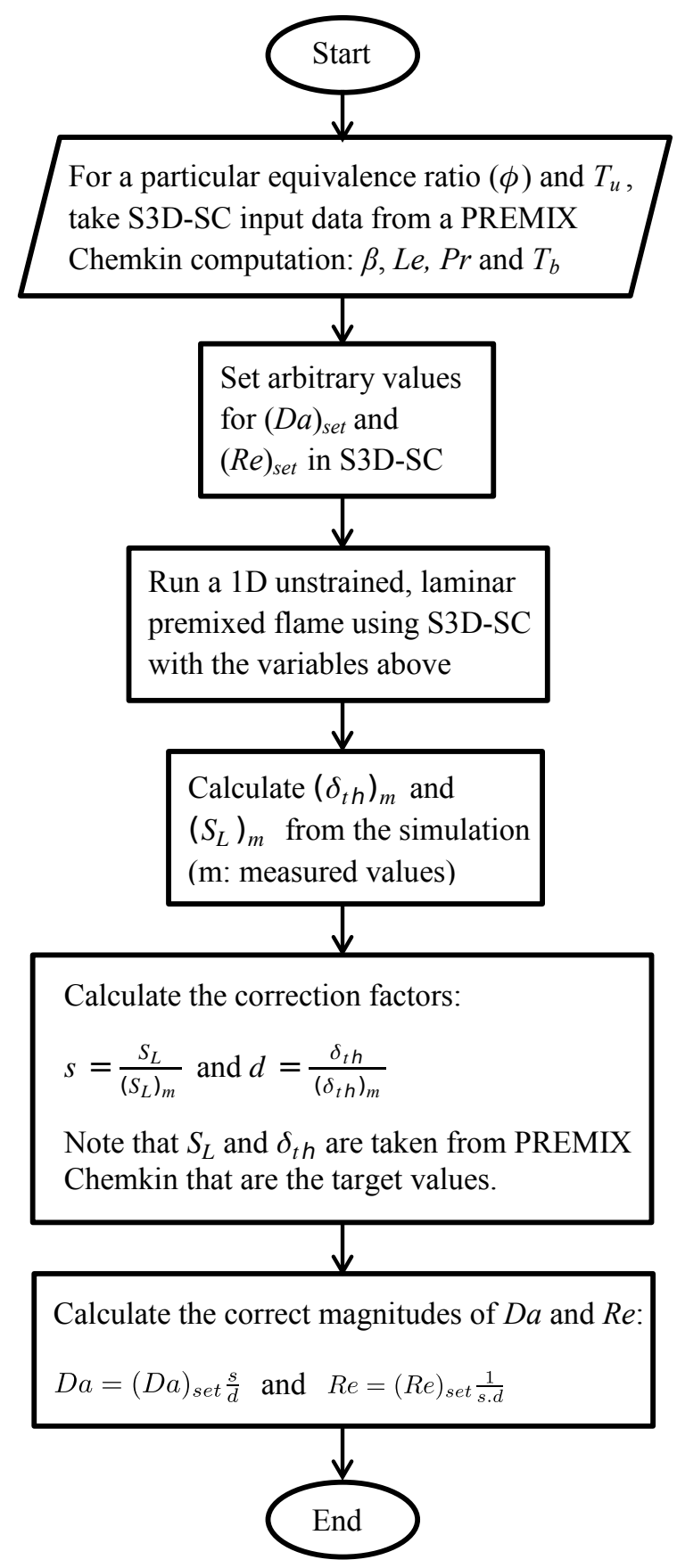

Figure 3.3: The procedures of determining the correct magnitudes of $D a$ (Damköhler number) and $R e$ (acoustic Reynolds number) to achieve the target values of $S_{L}$ and $\delta_{t h}$ in S3D-SC. 


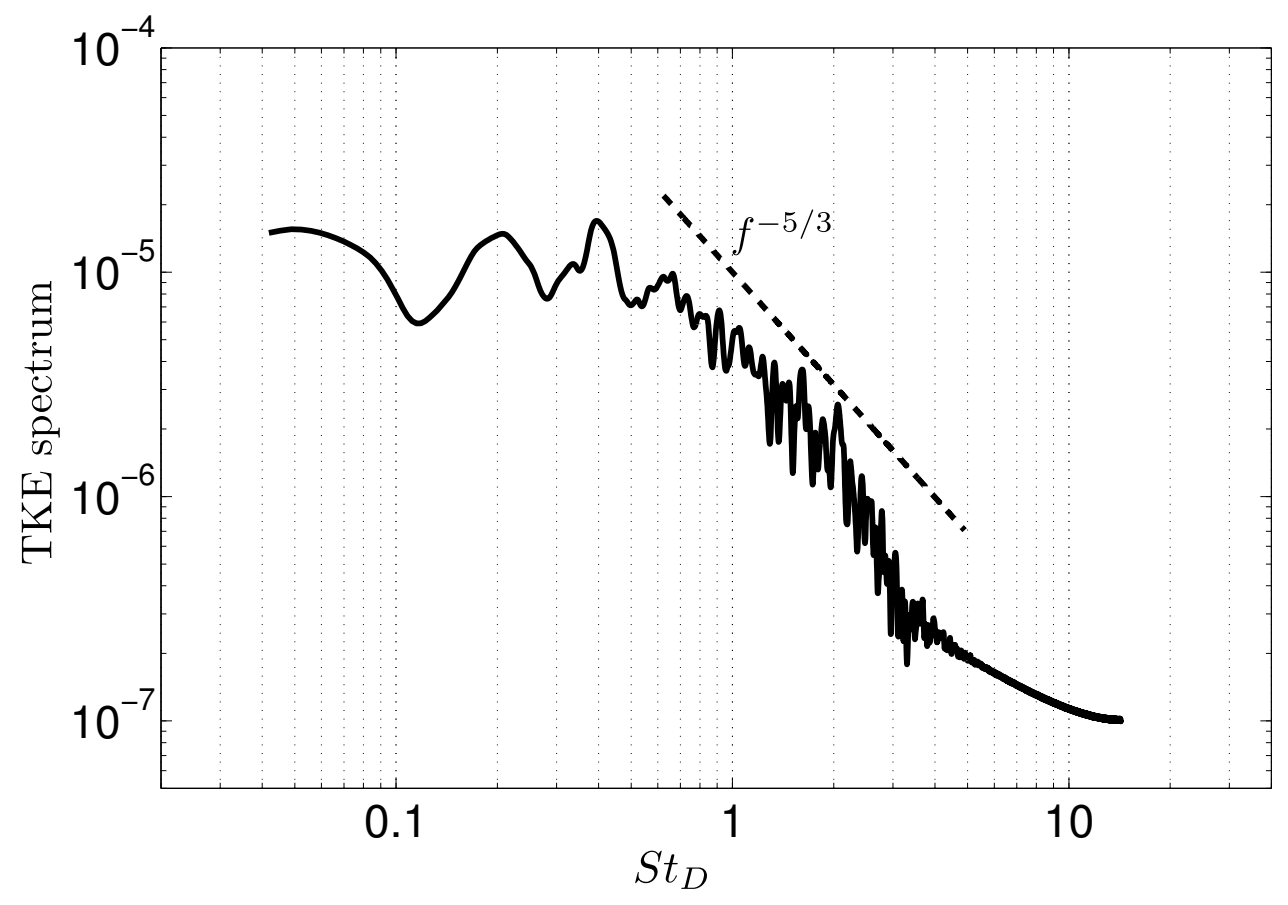

Figure 3.4: The inlet spectrum (solid line) of streamwise turbulent kinetic energy $\left(u^{\prime 2}\right)$ at the jet centreline. Note that the dashed line depicts the well-known $f^{-5 / 3}$ law for the kinetic energy spectrum in the inertial subrange.

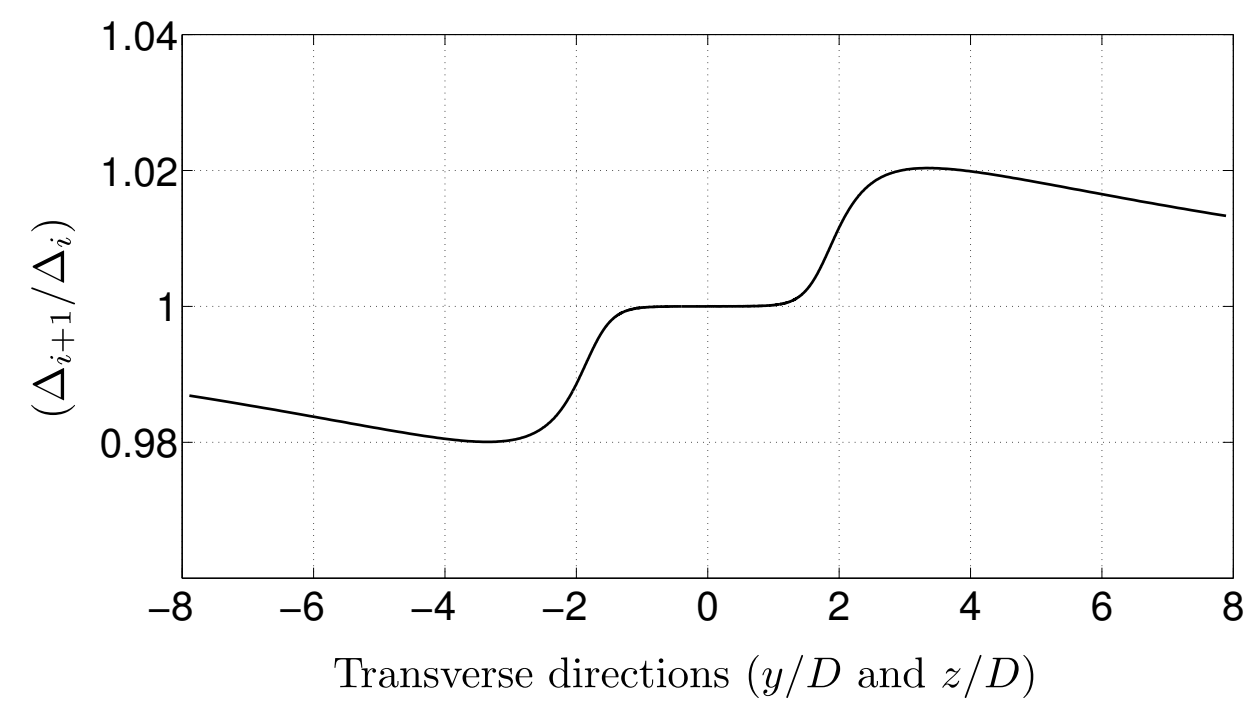

Figure 3.5: Grid stretching ratio in the transverse directions. 
(a)

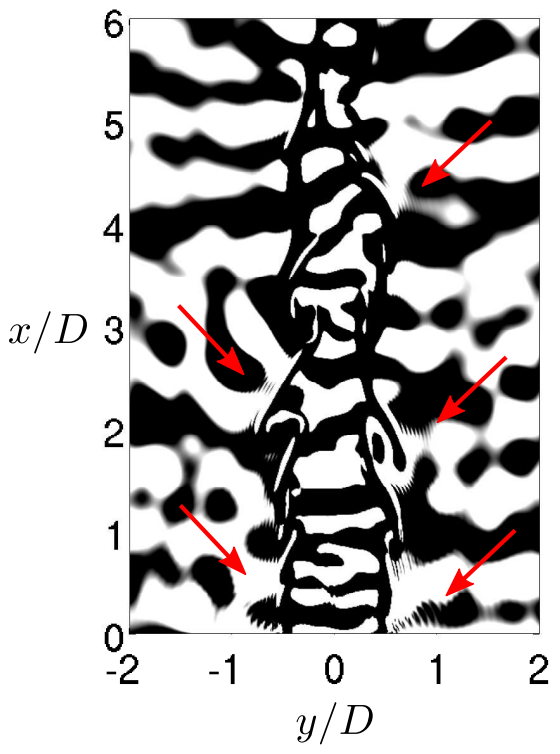

(c)

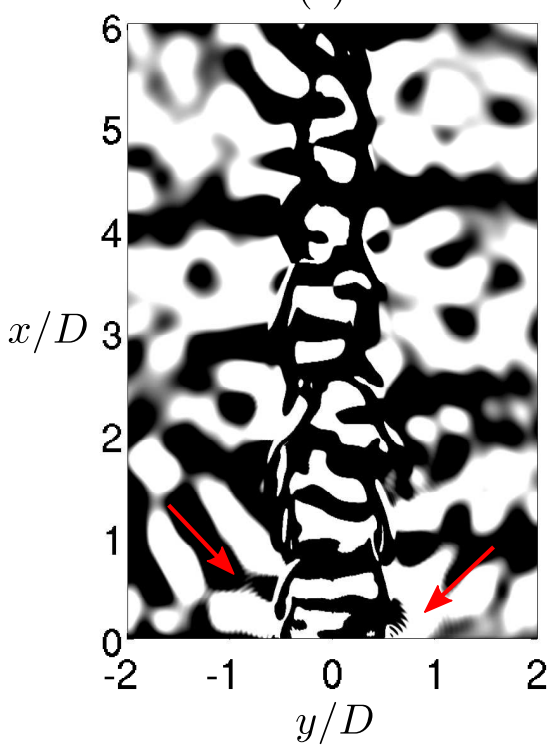

(b)

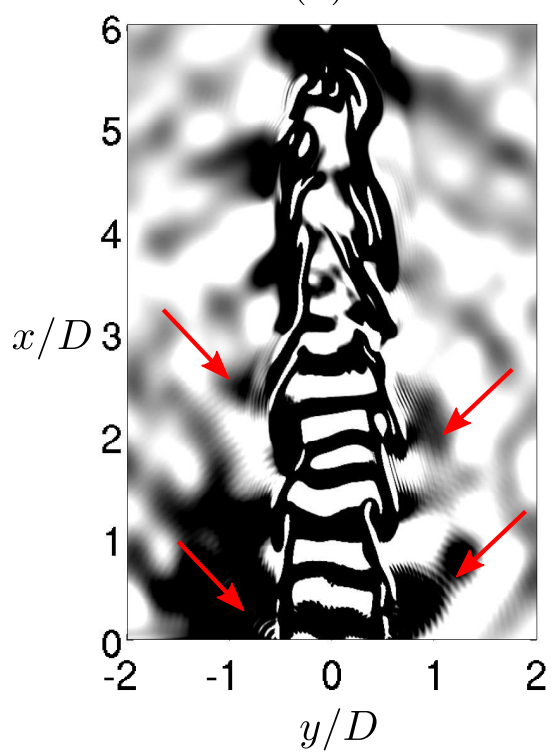

(d)

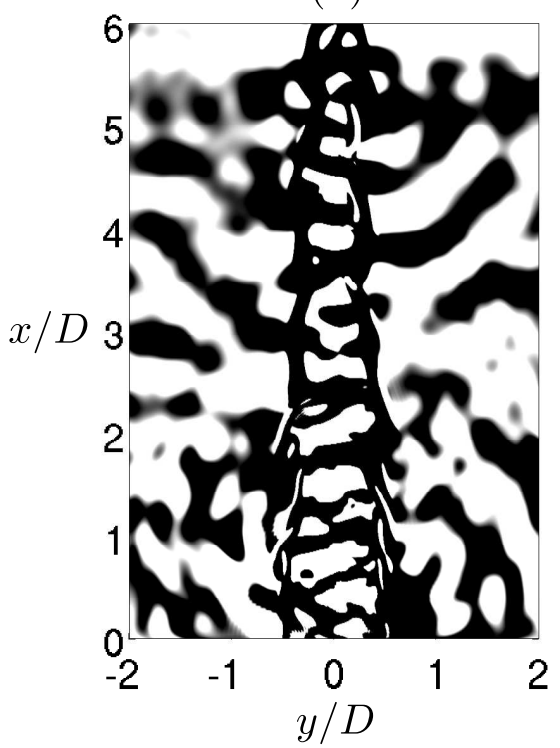

$$
\begin{array}{rllll}
\hline-1 & 0 & 1 & 2 & 3 \\
& & & & \times 10^{-4}
\end{array}
$$

Figure 3.6: Dilatation field on the jet central plane for four different mesh resolutions (grid points per jet diameter): (a) 114, (b) 142, (c) 185 and (d) 200. Occurrences of numerical noise are indicated. 
numerical noise at high frequencies, a 10th-order filter is also applied Kennedy et al., 2000 at every time step. Extensive resolution tests were performed to ensure that the internal structure of the flame was adequately resolved and the radiated sound was free of numerical noise. As demonstrated by Talei et al. 2014, the study of sound generation by premixed flames requires finer resolution than non-acoustic studies. Figure 3.6 shows the effect of various grid resolutions on the simulation results. The dilatation field - the divergence of velocity field and a sensitive quantity to numerical noise - was examined at the jet central plane. The number of grid points per jet diameter was varied from 114 to 200 . It is evident from this figure that the highest grid resolution (i.e. 200 grid points per $D$ ) leads to elimination of the high frequency, numerical noise that is present in the dilatation field of the coarser cases tested.

Figure $3.7 \mathrm{a}$ shows an instantaneous snapshot of the normalised temperature field $\theta$ at the plane $z=0$, with several cuts perpendicular to the flame front. $\theta$ is interpolated along these cuts, as illustrated in figure $3.7 \mathrm{~b}$. The number of grid points per flame thickness for the turbulent case is then calculated using equation 3.31 and figure 3.7b. This number varies between 9 and 14 grid points per $\delta_{t h} / D$, with $C$ attaining the maximum and $A$ attaining the minimum. Note there are also 10, 12 and 12 grid points across the flame for cuts $B, D$ and $E$, respectively. These numbers normally ensure a proper spatial resolution for a onestep chemistry DNS (e.g. Luo [1999]; Mehravaran \& Jaberi 2004]; Chakraborty \& Mastorakos 2006]; Nishiki et al. [2006]; Chakraborty \& Mastorakos [2008]; Chakraborty et al. 2010]; Karami et al. 2015.)

\subsubsection{Boundary Conditions and Sponge Layer Setup}

All inflow/outflow boundaries were carefully treated to avoid spurious noise reflections. So-called standard 'Navier-Stokes Characteristic Boundary Conditions (NSCBC)' Poinsot \& Veynante, 2005 were used at the outflow planes in the transverse directions. At the inflow boundary and the streamwise outflow plane the modified NSCBC known as '3DNSCBC' [Yoo \& Im, 2007] were used, accounting for the effect of transverse terms. It should be noted that no treatment was implemented for edges and corners since they are quite far from the main 
(a)

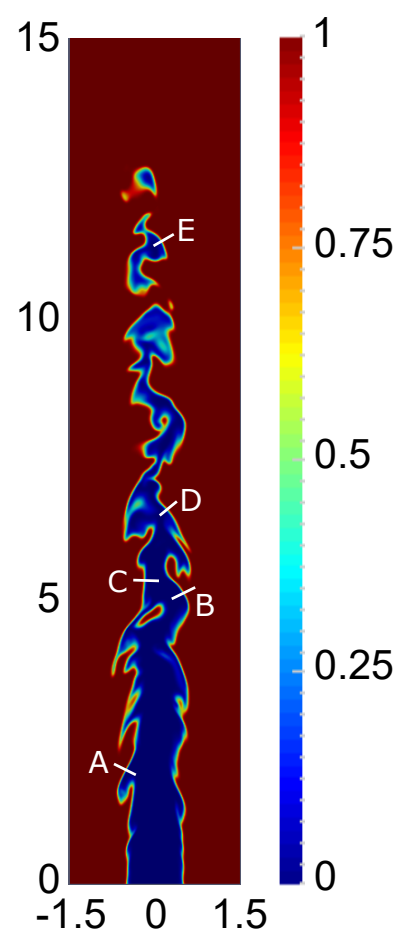

(b)

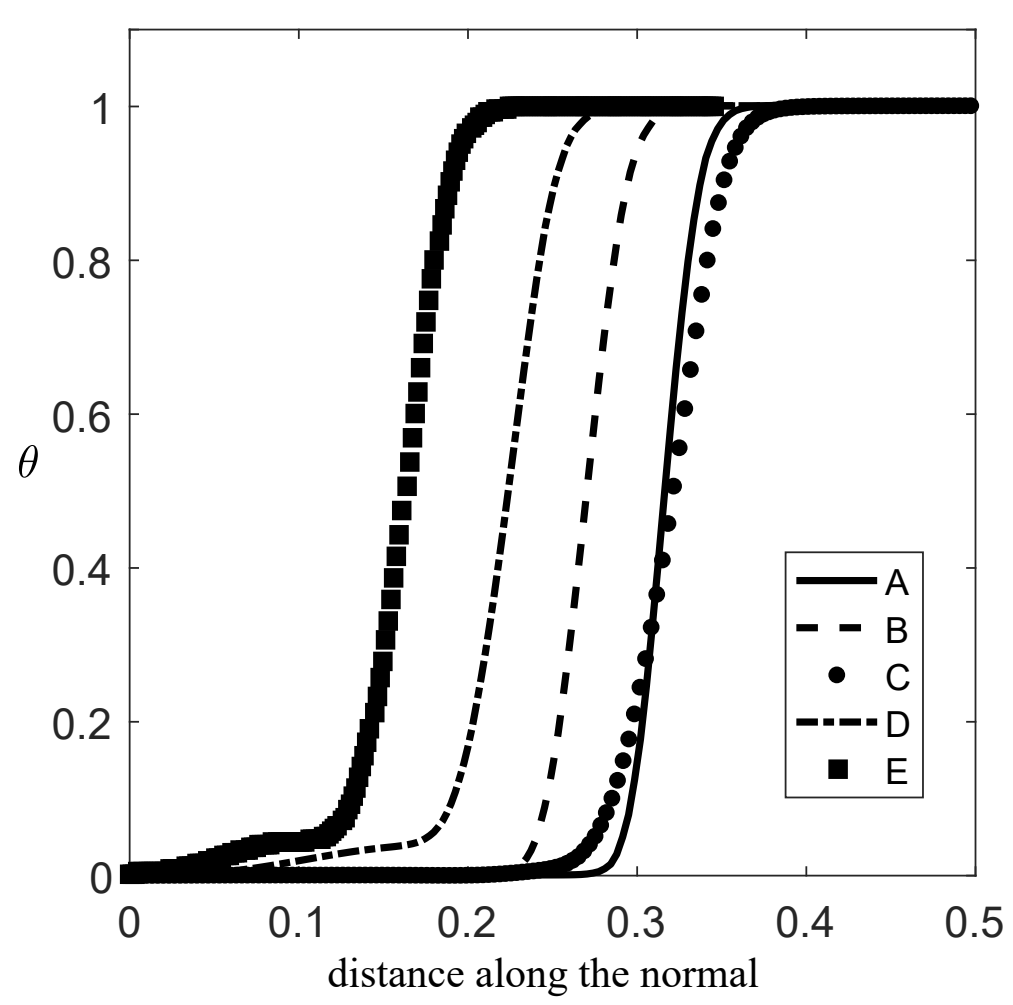

Figure 3.7: (a) An instantaneous snapshot of the normalised temperature field $\theta$ at the plane $z=0$. (b) $\theta$ interpolated along the lines A-E indicated in (a).

flow field, and therefore their effects were found to be minimal by examining the simulations at those regions. A sponge layer Bogey et al., 2000; Colonius et al., 1997 was used for each case in the streamwise direction from $20 D$ to $25 D$ to gradually damp the velocity fluctuation at the outflow boundary. The mesh was also slightly stretched in the axial direction in this zone. In the sponge layer, the RHS of the governing equations was modified so that the solution is driven towards a given steady target solution $\mathbf{U}_{\mathbf{0}}$ according to

$$
\frac{\delta \mathbf{U}}{\delta t}=\ldots-\sigma(x)\left(\mathbf{U}-\mathbf{U}_{\mathbf{0}}\right),
$$

with Bogey et al. 2000

$$
\sigma(x)=\sigma_{\max }\left(\frac{x-x_{0}}{x_{\max }-x_{0}}\right)^{2},
$$



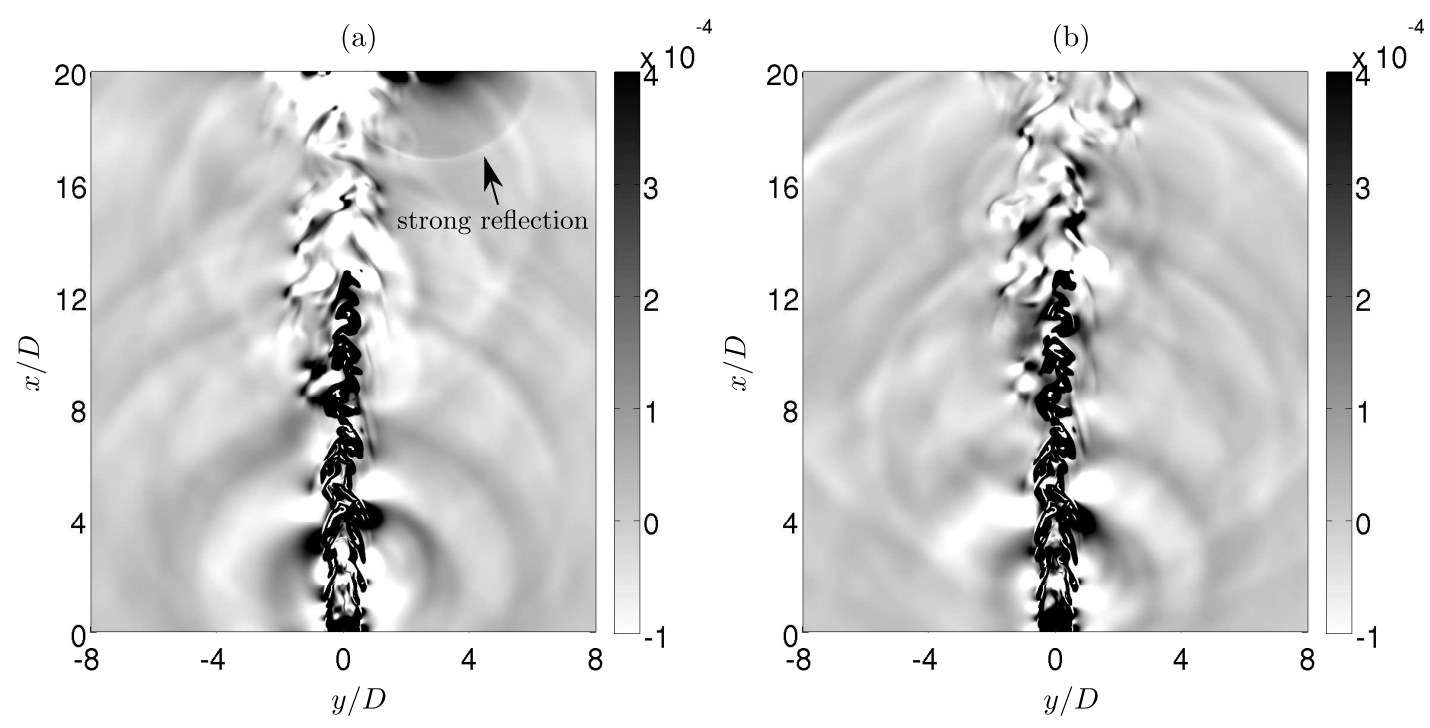

Figure 3.8: Comparison of the dimensionless dilatation field $\nabla \cdot \vec{u}$ at the jet central plane (a) without and (b) with the sponge layer.

where $\sigma_{\max }=0.1$ and $x_{0}$ and $x_{\max }$ correspond to the start and end of the sponge layer, respectively. It should be noted that in the damping zone the target temperature and reactant species mass fraction are equal to the adiabatic flame temperature and zero, receptively. The target velocity profile was obtained from the self-similar solution of round jet flows (Hussein et al. [1994) ):

$$
\frac{U(x, r)}{U_{c}(x)}=\exp \left[-C_{0}\left(\frac{r}{B_{0} x}\right)^{2}\right]
$$

with

$$
U_{c}(x)=A_{0} \frac{U_{a v}}{x},
$$

where $C_{0}=0.693$. The decaying coefficient $\left(A_{0}\right)$ and the spreading factor $\left(B_{0}\right)$ were obtained using the time average velocity field of a case without the sponge layer (i.e. $\sigma_{\max }=0$ ).

As observed in figure 3.8 , the implemented sponge layer clearly avoids spurious noise reflection from the streamwise outflow boundary into the computational domain, while a strong noise reflection is seen for the case without the sponge layer. As can be seen in figure 3.9, the carefully chosen sponge layer parameters have a negligible effect on the upstream flow field. It should be also noted that 


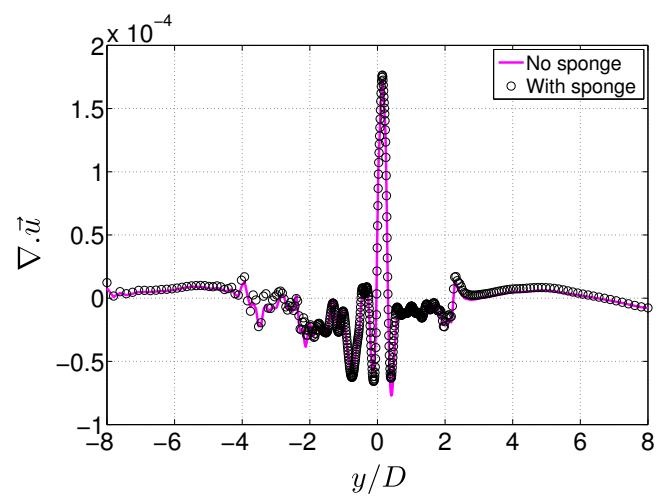

(a)

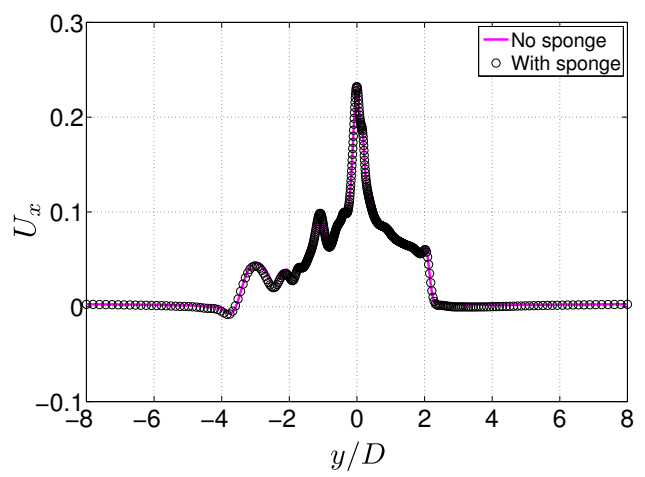

(b)
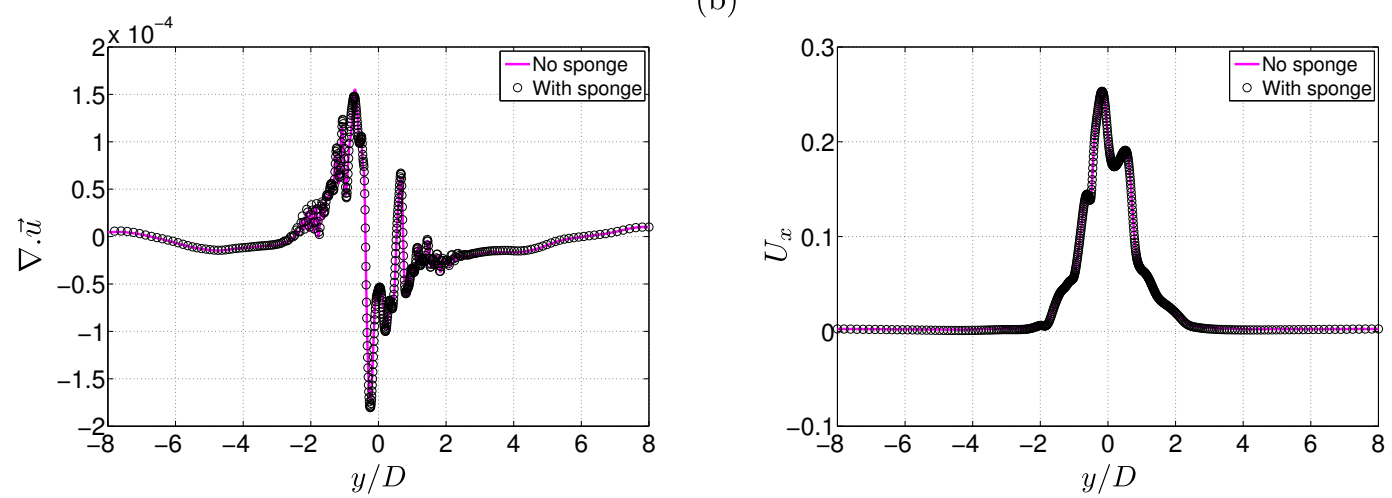

Figure 3.9: Comparison of the dimensionless dilatation field $\nabla \cdot \vec{u}$ (left) and the streamwise velocity field $U_{x}$ (right) at two different axial locations without (solid line) and with (circle) the sponge layer implemented: (a) close to the start of the sponge layer and (b) $2 D$ upstream of the start of the sponge layer.

the DNS results for both cases with the sponge layer were carefully monitored and no noise reflection was observed from the boundaries.

\subsubsection{DNS Run and Computational Cost}

The simulations were performed using a constant time step for 6 jet flow-through times $\tau_{j}=L_{x} / U_{j}$, and only the data from the last $4 \tau_{j}$ were used for postprocessing. The time history of minimum and maximum pressure in the whole computational domain were recorded during the simulation and no pressure drift was observed, (see figure $3.10 \mathrm{a}$ ). To ensure that all initialisation effects were also decayed and a statistically stationary solution was achieved, the time history of 


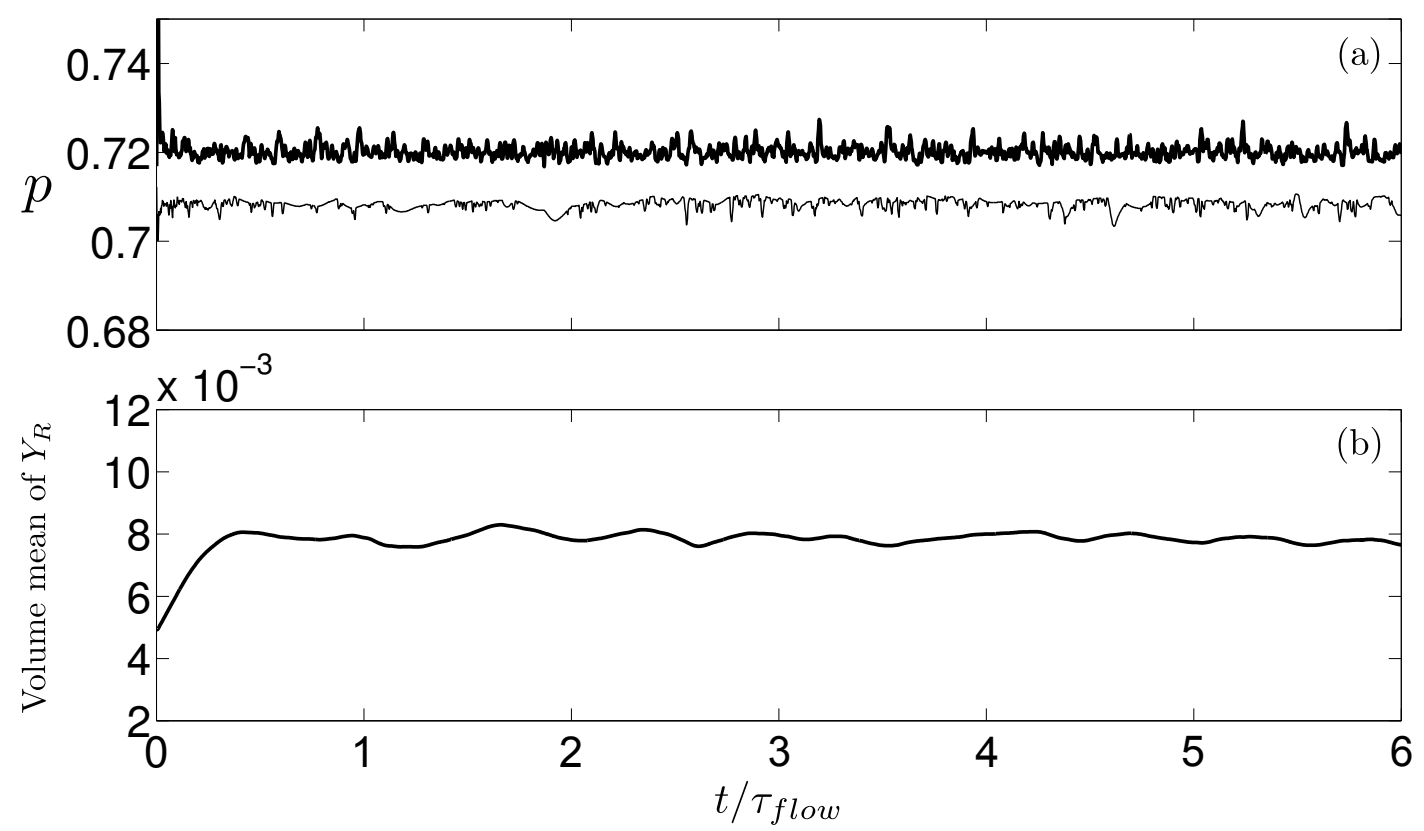

Figure 3.10: (a) The normalised time history of (a) the minimum (thin solid) and the maximum (thick solid) pressure in the whole computational domain; and (b) the volume mean of reactant species mass fraction $\left(Y_{R}\right)$. The result of the stoichiometric case is shown here.

the volume mean of reactant species mass fraction $\left(Y_{R}\right)$ was also tracked (c.f. figure 3.10b). Overall, the computational costs for the stoichiometric and lean cases were equal to 1.4 and 1.9 million CPU-hours, respectively, running on 9216 computing cores (Intel Xeon E5-2690V3 "Haswell"processors, 2.6 GHz). 


\subsection{Summary}

This chapter presented the numerical methods and the configuration which were used to study noise generation by turbulent, premixed jet flames. Direct Numerical Simulation (DNS) was used in this study. An introduction of the governing equations of reacting flows with a single-step, irreversible chemistry model was expressed, followed by the details of the boundary conditions and the DNS solver used. Finally, the computational domain and the DNS setup were discussed. 


\section{Chapter 4}

\section{DNS of Sound Generation by Turbulent Premixed Flames}

\subsection{Introduction}

This chapter presents three-dimensional Direct Numerical Simulations (DNS) of sound generation by open turbulent premixed flames. A simple chemistry model is used, since the computational cost for the study of sound generation by turbulent, premixed flames is significantly higher than for non-acoustic studies of combustion Talei et al., 2014]. Two turbulent, methane-air round jet flames are considered with different equivalence ratios $(\phi=0.7$ and 1.0), and the premixture preheated to $T_{u}=800 \mathrm{~K}$. The structure of both flames is first investigated. Key DNS results are then presented. This sheds light on the significance of flame annihilation events in terms of the sound generation by turbulent premixed flames. Finally, the spectral content of the far-field radiated sound is also compared to experimental studies performed by Rajaram \& Lieuwen 2009].

\subsection{General Structure}

The present DNS conditions are shown on the regime diagram for premixed turbulent combustion Peters, 2000 as depicted in figure 4.1. As can be seen, both cases are in the thin reaction zones regime. Note that the turbulence length scale 


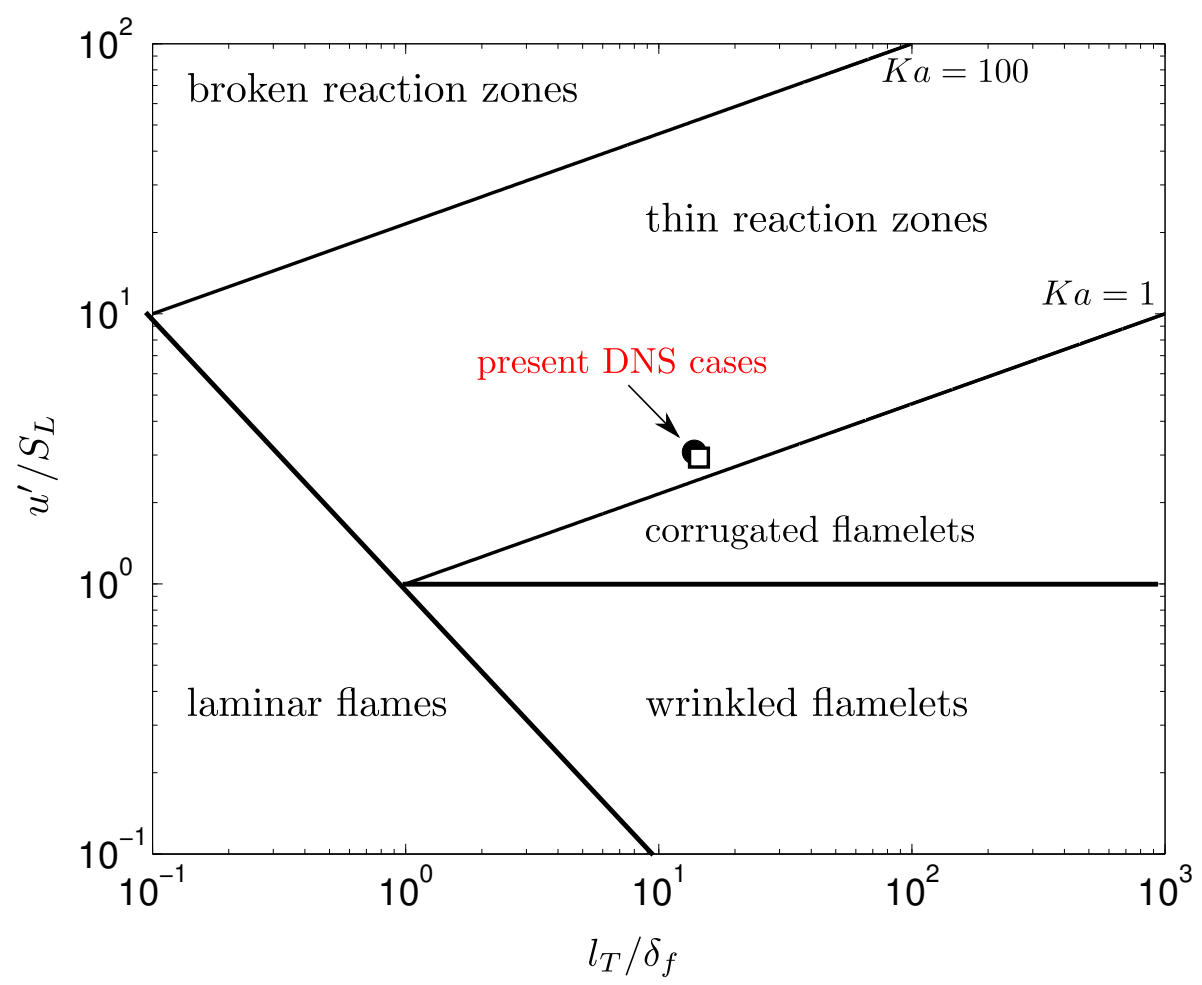

Figure 4.1: The locations of the present DNS cases on the regime diagram for premixed turbulent combustion: $\phi=1.0(\bullet)$ and $\phi=0.7(\square)$.

$l_{T} / D$ is the longitudinal integral length scale, and is equal to 0.3 for both cases (see table 3.1), and $u^{\prime}$ is the root-mean-square (r.m.s) of velocity fluctuation evaluated on the jet centreline at the jet exit (see table 3.1) Gülder \& Smallwood, 2007]. Further, the flame length scale $\delta_{F} / D$ is given in non-dimensional form by

$$
\frac{\delta_{f}}{D}=\frac{1}{R e S c\left(S_{L} / c_{u}\right)}
$$

where $\delta_{f}$ is the nominal (Zel'dovich) flame thickness Gülder \& Smallwood, 2007; Tamadonfar \& Gülder, 2015] determined as the ratio of mass diffusivity of the unburned mixture to the laminar flame speed. It should be noted that the nominal flame thickness $\left(\delta_{f}\right)$ is different from the laminar flame thickness $\left(\delta_{t h}\right)$ estimated as the thermal thickness (see equation 3.31). In this regime, the Kolmogorov length scale is smaller than the flame thickness but bigger than the reaction zone 

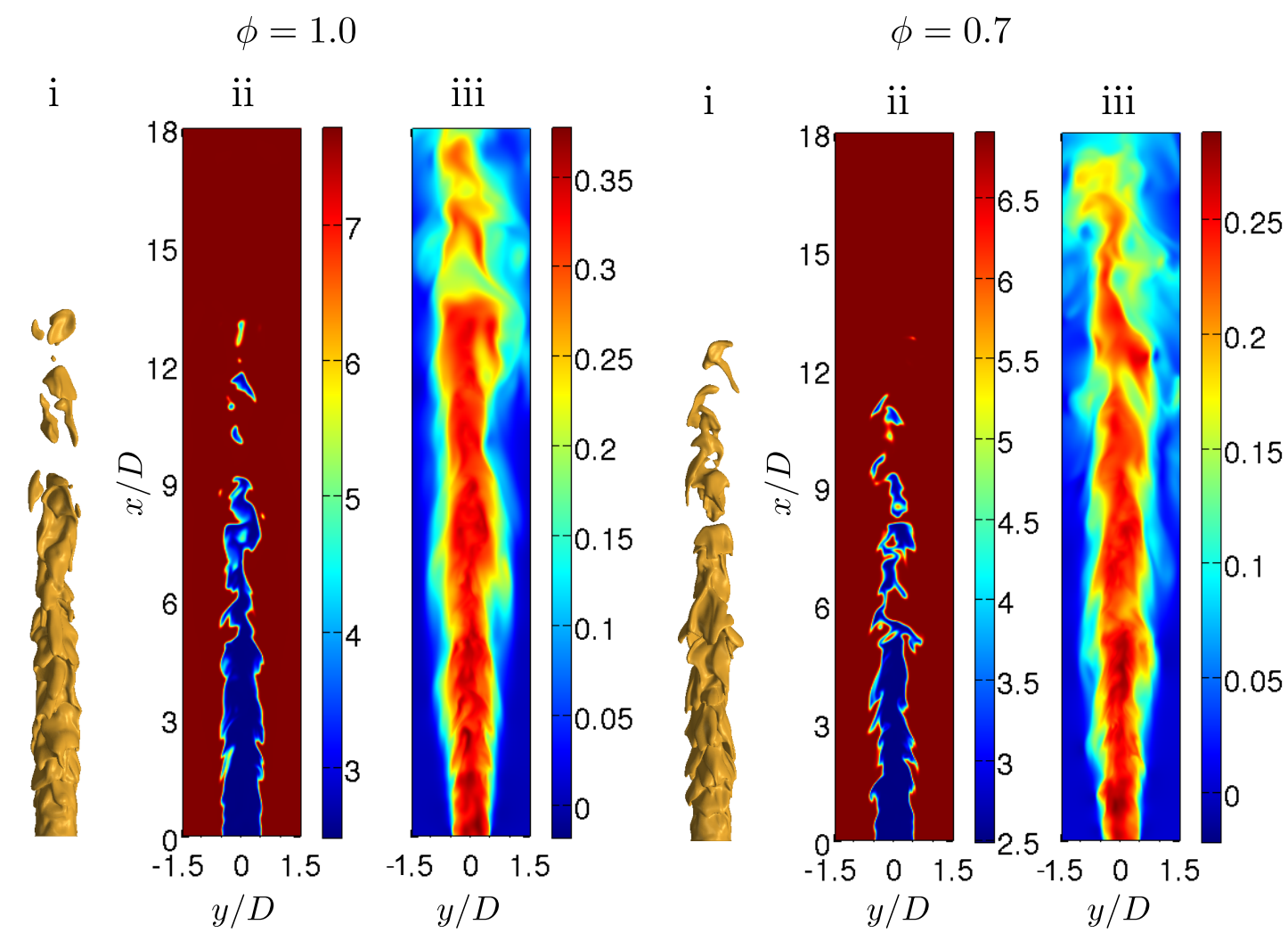

Figure 4.2: Instantaneous snapshots of (i) the flame iso-surface (ii) the temperature field $T$ and (iii) the streamwise velocity field $U_{x}$ extracted at the central plane through the jet: $\phi=1.0$ (left) and $\phi=0.7$ (right).

thickness so that the smallest turbulent eddies can penetrate into the preheat zone but not into the reaction zone. Therefore, alteration of the flame inner structure will not occur by Kolmogorov eddies.

Figure 4.2 shows the flame general structure for both cases. To represent the flame surface, iso-surfaces of the progress variable with $Y_{R}=0.122$ and 0.156 for $\phi=1.0$ and 0.7 are respectively considered. These iso-levels are selected because the corresponding progress variable for each case is close to the point of peak heat release in a one-dimensional, unstrained premixed flame using the same chemical parameters as the turbulent cases. Figures 4.2 ii and 4.2 iii also depict the corresponding snapshots of the temperature field and streamwise velocity field, respectively, extracted at the central plane through the jet at this instant. As can be seen, the instantaneous flame brush and the jet main development entirely 
(a) $\phi=1.0$

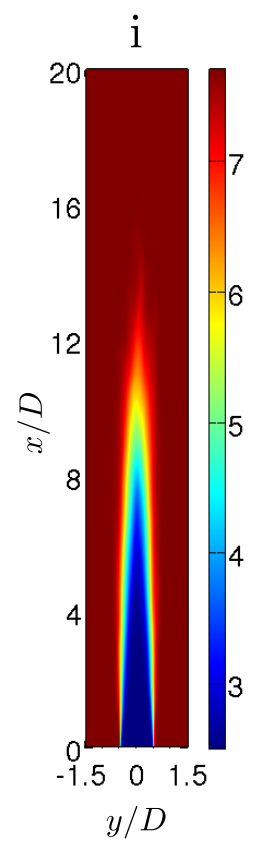

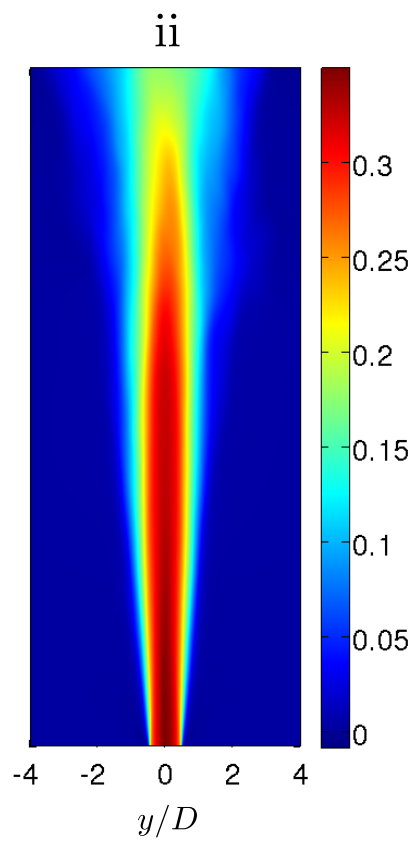

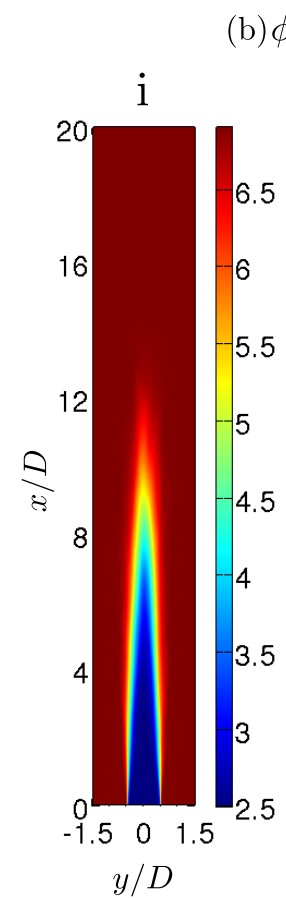

(b) $\phi=0.7$

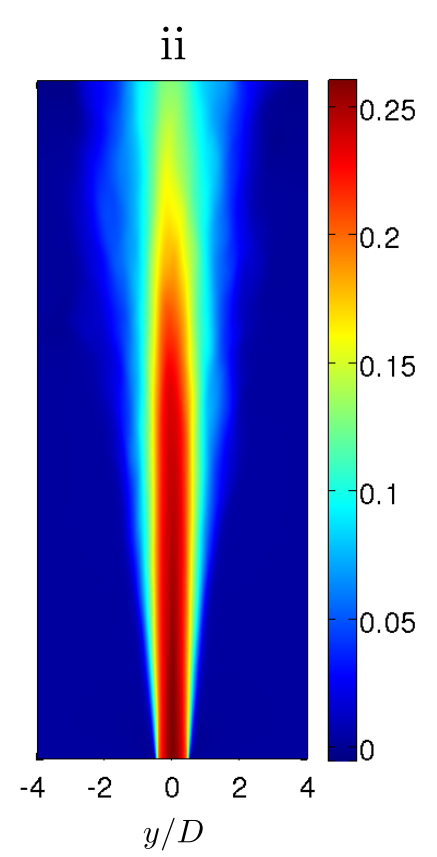

Figure 4.3: The time averaged (i) temperature field $\bar{\theta}$ and (ii) streamwise velocity field $\bar{U}_{x}$ at the jet central plane: (a) $\phi=1.0$ and (b) $\phi=0.7$.

remains within the fine-mesh region $(|y, z| \leq 1.5 D$ and $0 \leq x \leq 20 D)$ for each case. A visual inspection of figure 4.2 suggests that the flame surface in the lean case experience a higher level of turbulence compared to the stoichiometric case. One of the reasons for the observation could be a lower viscosity in the burned gas caused by the lower temperature ratio for the lean flame (see equation 3.9). As discussed by Poinsot \& Veynante 2005] increased kinematic viscosity associated with temperature increase can weaken the turbulence.

Figure 4.3 shows time averages of the temperature field $\bar{\theta}$ and the streamwise velocity field $\bar{U}_{x}$ at the jet central plane for both cases. Overall, the lean flame appears to be shorter than the stoichiometric flame. Moreover, the main developments of the jets follow nearly similar patterns. This becomes obvious where the jet half-width $r_{1 / 2}(x)$ (a commonly used measure to characterise the growth of a jet, defined as the distance from the centreline of the jet where the local mean velocity is equal to half of the local centreline mean velocity) of both cases is compared in figure 4.4. This is not surprising given that both jets feature the 


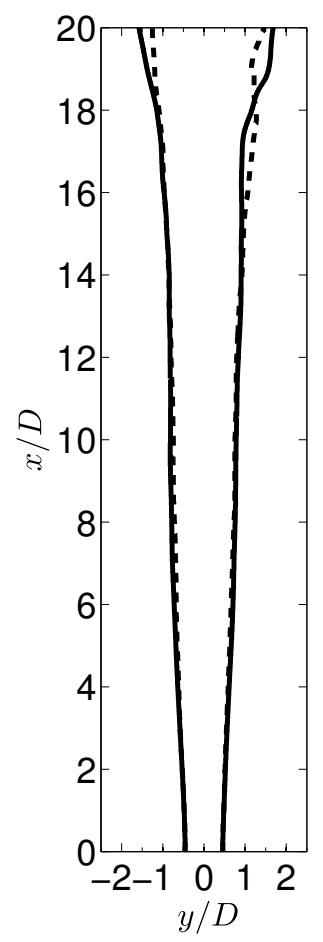

Figure 4.4: Jet half-width $r_{1 / 2}(x): \phi=1.0$ (solid) and $\phi=0.7$ (dashed)

same jet Reynolds number.

Figure $4.5 \mathrm{a}$ shows the time averages of the normalised temperature and the normalised streamwise velocity field conditioned on the radial distance $r / D$ at three different streamwise locations $(x / D=2,10$ and 18$)$ for both cases. Note that, the RMS of the fluctuating parts of these variables are also depicted in figure $4.5 \mathrm{~b}$. As can be seen, both cases are similar in terms of the mean and RMS profiles. Once again this is consistent with what was observed in figures 4.4 and 4.3. Given the observed similarity between the two cases, any difference in their acoustic fields can arguably be related to their difference in chemical parameters.

Figure 4.6 illustrates time evolution of the flame surfaces for both cases. As can be seen, the flame brush has a complex structure, experiencing frequent annihilation events in particular close to the flame tip. These flame annihilation events may potentially contribute to the noise generated by these turbulent premixed flames as will be discussed later in this chapter. 
a)
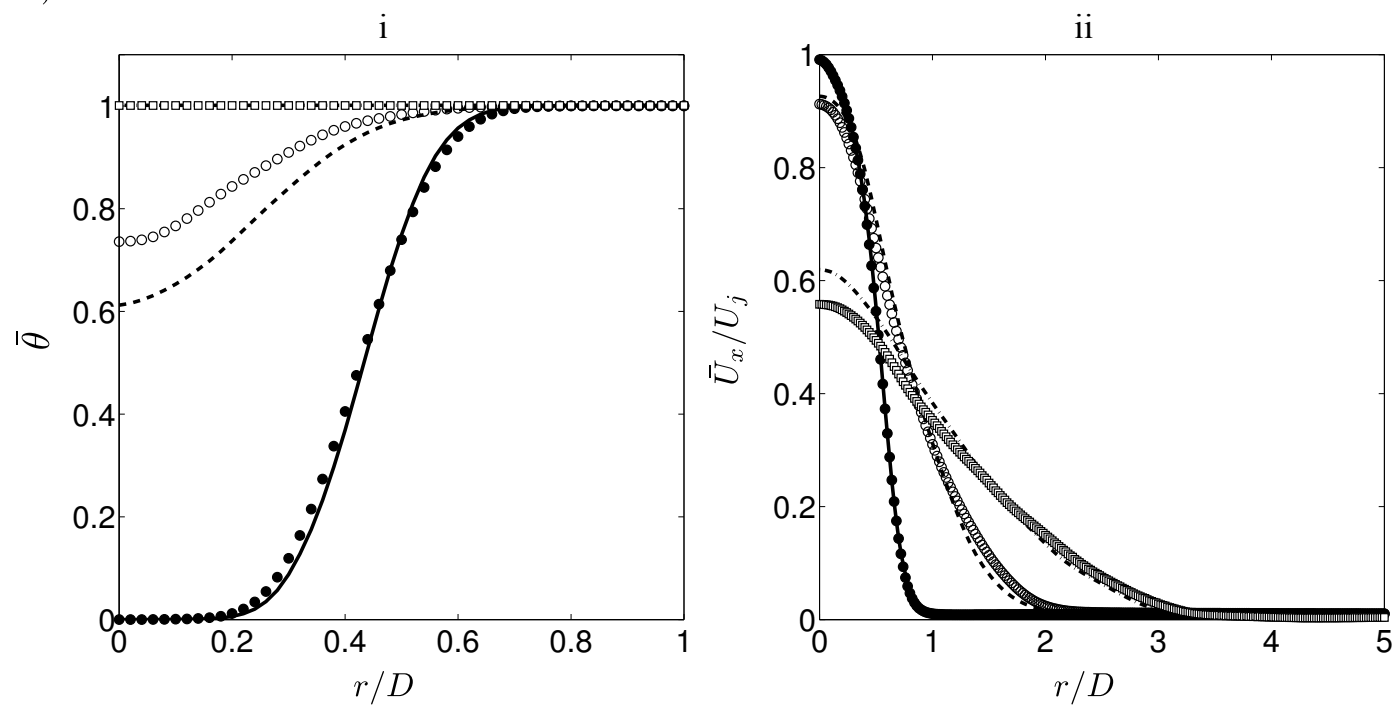

b)
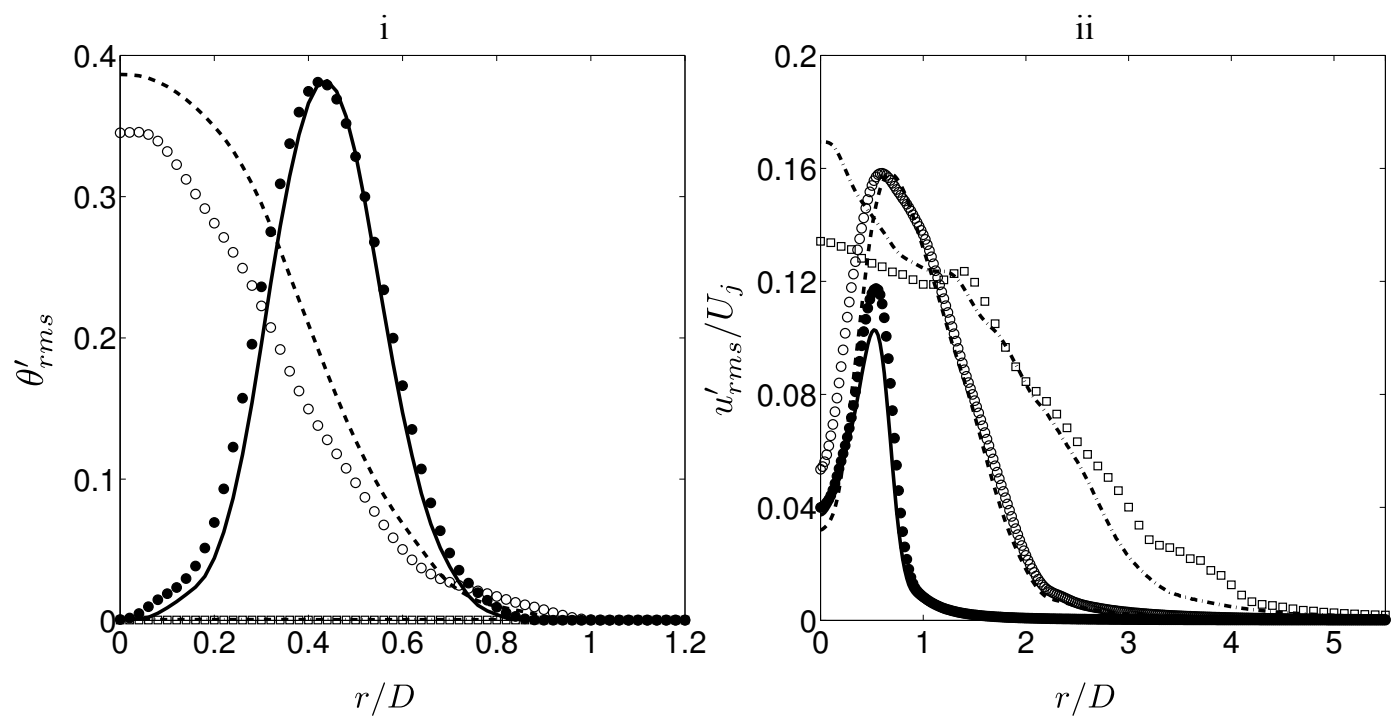

Figure 4.5: (a) Time averages and (b) RMS of the fluctuations of (i) the normalised temperature field and (ii) the normalised streamwise velocity field conditioned on $r / D$ at three different streamwise locations: $x / D=2$ (solid line $\phi=1.0$ and solid circle $\phi=0.7$ ), $x / D=10$ (dashed $\phi=1.0$ and hollow circle $\phi=0.7$ ) and $x / D=18$ (dash-dot $\phi=1.0$ and hollow square $\phi=0.7$ ). 
(a) $\phi=1.0$

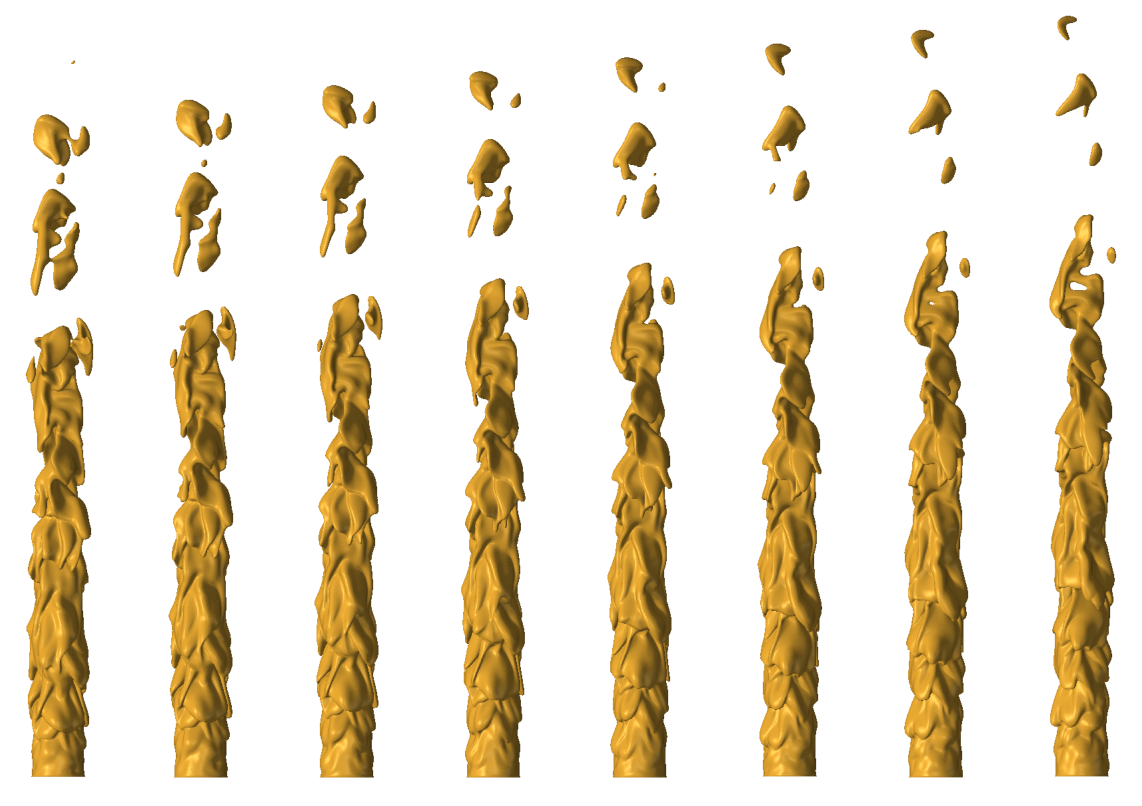

(b) $\phi=0.7$

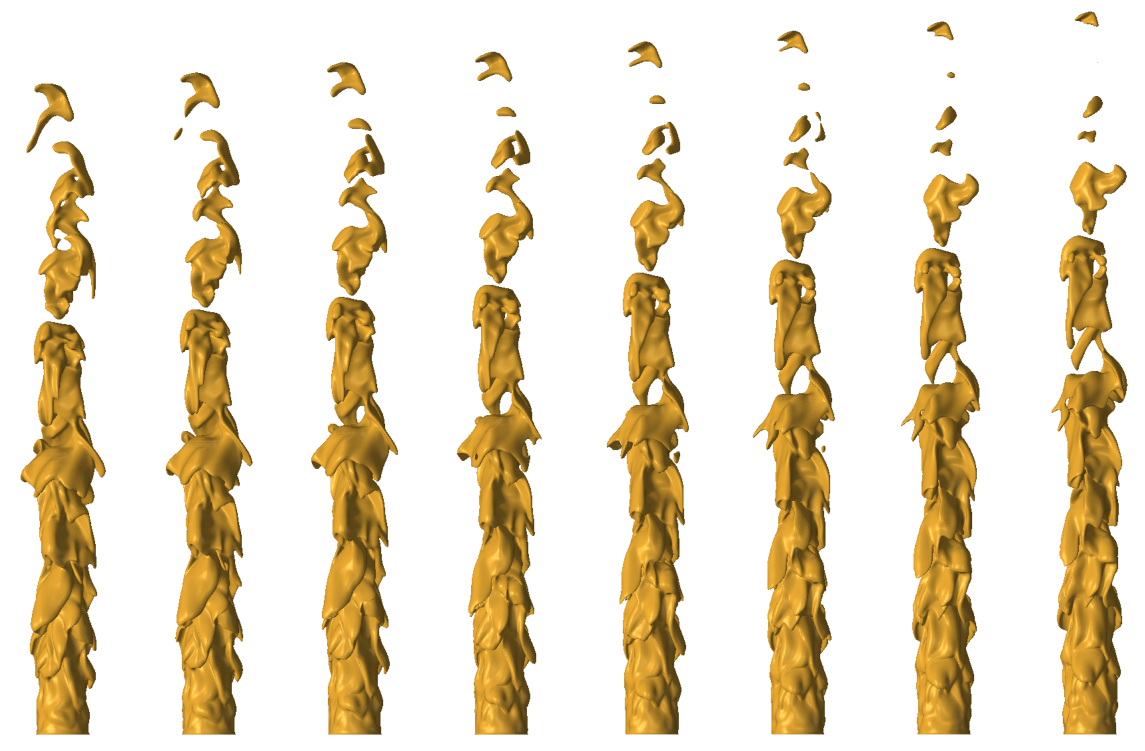

Figure 4.6: Instantaneous iso-surfaces of the progress variable $Y_{R}=0.122$ and $Y_{R}=0.156$ for (a) $\phi=1.0$ and (b) $\phi=0.7$, respectively. 


\subsection{Sound Generation}

Dilatation field $\nabla \cdot \vec{u}$ is commonly used in aero-acoustics to identify sound (e.g. Colonius et al., 1997; Talei et al., 2014, 2012b ). It can be readily shown that the dilatation and the pressure are related in the far field [Colonius et al., 1997] through the following equation:

$$
\frac{\partial p}{\partial t}+\rho_{\infty} c_{\infty}^{2} \nabla \cdot \vec{u}=0
$$

Figure 4.7 and 4.8 show instantaneous snapshots of the dimensionless dilatation field on the central $x$-y plane through the jet for the stoichiometric and the lean flame respectively. The acoustic fields for both cases clearly feature monopolar sound sources originating from the flame. However, weaker monopolar sources appear in the lean flame. As discussed earlier, since both cases experience almost the same level of turbulence, this observation is likely related to a difference in chemical parameters between the two cases.

To shed light on the source of the observed monopolar sound waves, three examples labelled as A1, A2 and A3 in figure 4.7 are chosen. Figure 4.9 shows the flame iso-surface superimposed on the dilatation field, highlighting the origin of the observed waves. As can be seen, the event A1 involves consumption of a pocket of unburned gases in the downstream region near the flame tip. Consistent with the earlier works of our group [Talei et al., 2014, 2012b], this phenomenon is referred to as 'flame island burn-out'. Event A2 is referred to as a 'flame pinchoff', with a pocket of unburned gases locally detaching from the flame surface. Finally, the event A3 takes place when flame-to-flame interaction first results in the formation of a tunnel of burned gas, and then the local flame area is lost due to cusp retraction associated with another pinch-off event. We call this first event 'tunnel formation'. The topology of these events has been studied recently by Griffiths et al. [2015], although they use slightly different language to classify them. The observed sound waves in figure 4.7 appear to be in significant part due to these frequent flame annihilation events. It is important to note that the significance of the flame annihilation events to noise generation by turbulent premixed flames is presented in this DNS study. This was not previously shown, as the focus of earlier studies was placed on sound generation by laminar premixed 

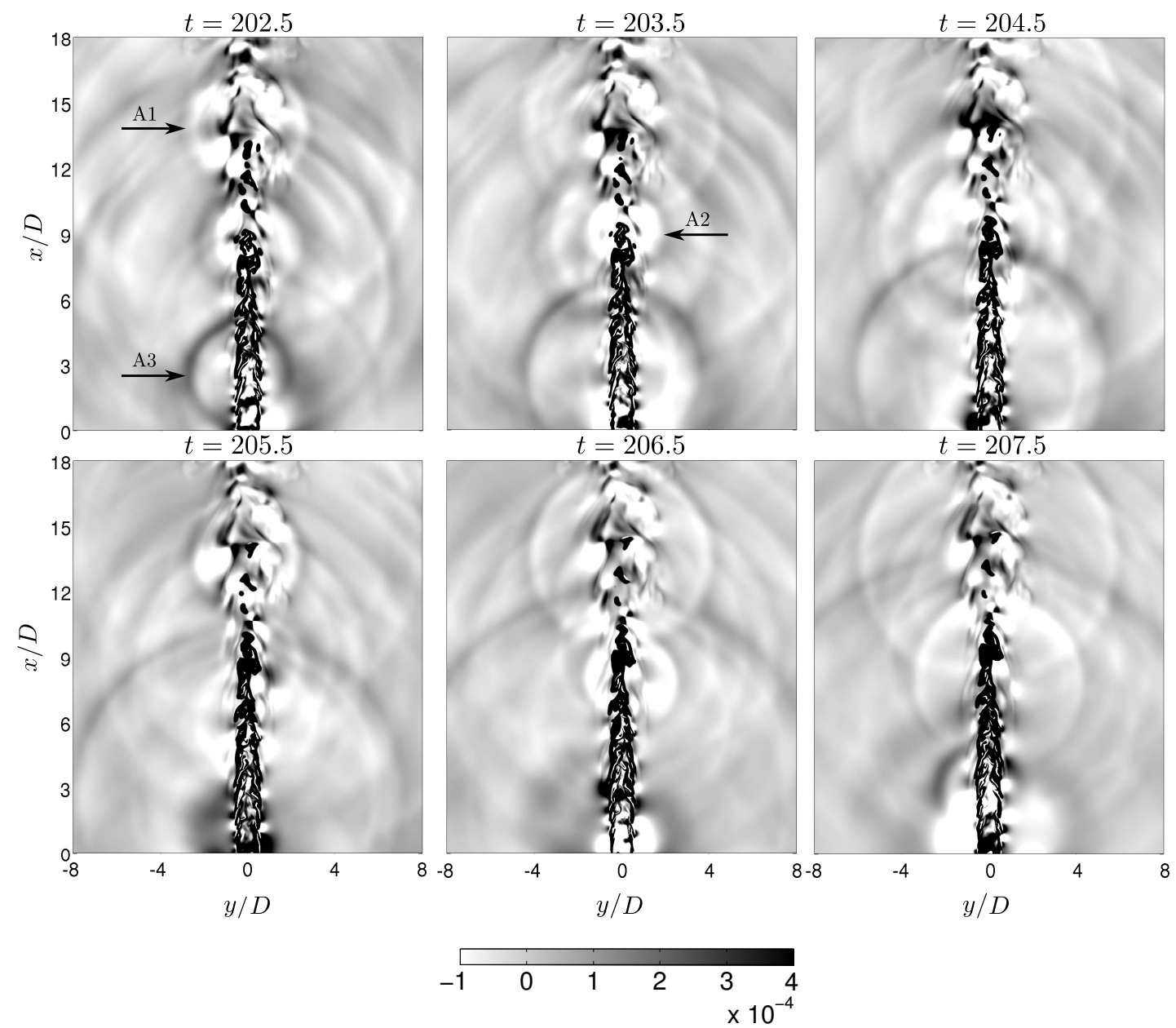

Figure 4.7: Instantaneous snapshots of the dimensionless dilatation field $\nabla \cdot \vec{u}$ on the jet central plane for $\phi=1.0$.

flames, e.g. Kidin et al. [1988]; Schuller et al. 2003]; Candel et al. [2004]; Talei et al. 2011, 2012a, 2014, 2012b]; Brear et al. [2012]; Jiménez et al. [2015].

\subsection{Spectral Analysis}

Rajaram \& Lieuwen 2009] showed that a peak Strouhal number $S t_{\text {peak }}=f_{\text {peak }}$ . $L_{\text {flame }} / U_{a v}$ of the far-field spectrum was always in order of unity and varied between 0.5 and 2.2 across a large set of turbulent premixed flames tested, where $L_{\text {flame }}$ was a flame length and $U_{a v}$ was the cold flow, bulk jet velocity. It should, 

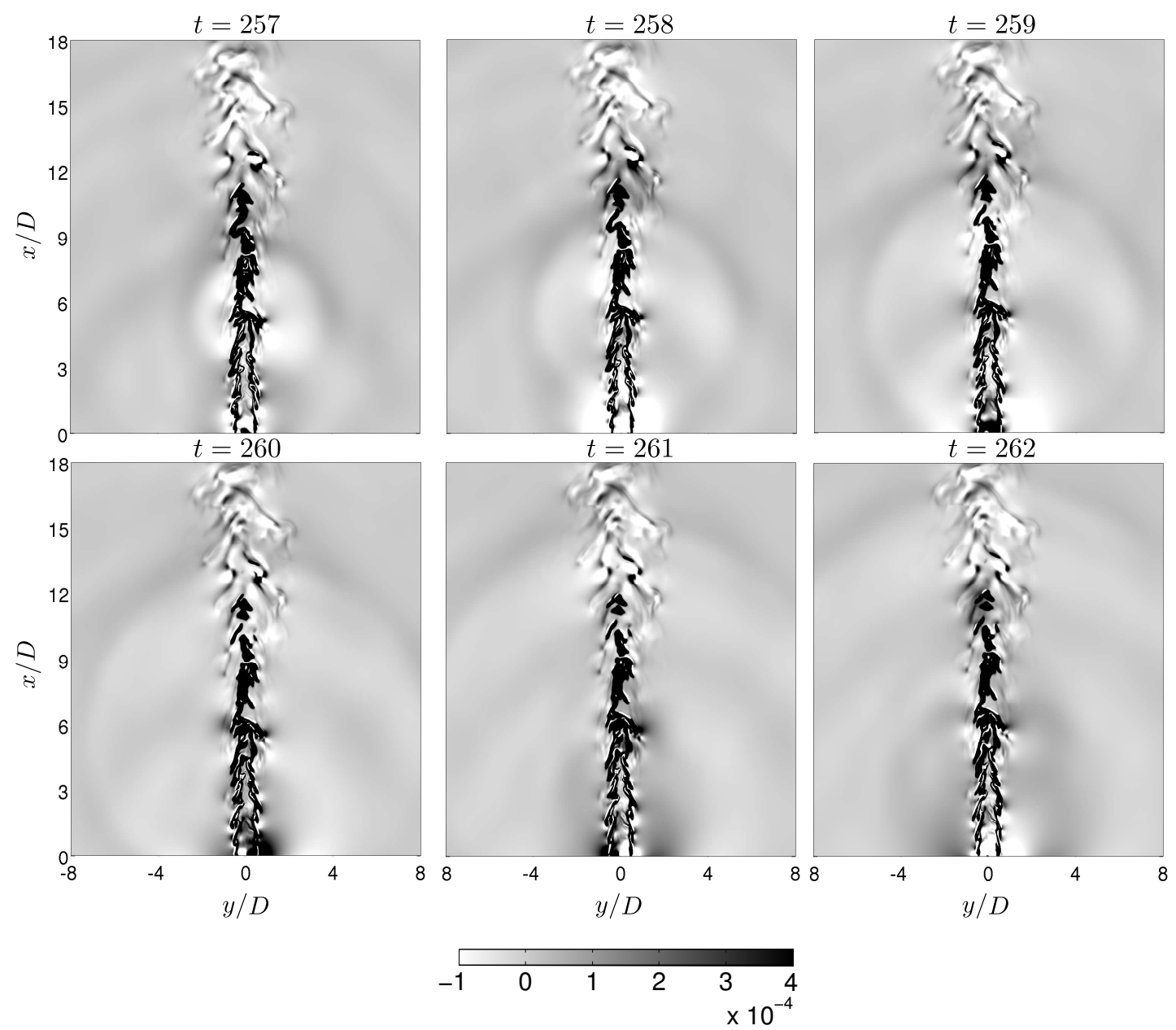

Figure 4.8: Instantaneous snapshots of the dimensionless dilatation field $\nabla \cdot \vec{u}$ on the jet central plane for $\phi=0.7$.

however, be noted that Rajaram \& Lieuwen 2009] calculated their flame length using the variance in the intensity of chemiluminescence emitted by the flame at a given point in space. The flame length $L_{\text {flame }}$ was then defined as the distance between the heights where the horizontally integrated intensity of the variance image crosses a quarter of its maximum value. In the present study, the flame length was therefore calculated using the variance of the reaction rate field. As depicted in figure 4.10 , these flame lengths were approximately equal to $13 D$ and $12 D$ for the stoichiometric and lean cases, respectively.

The spectrum of the produced sound is calculated on a plane perpendicular to the jet axis at three different streamwise locations $x / D(=2,10$ and 18) using 
A1

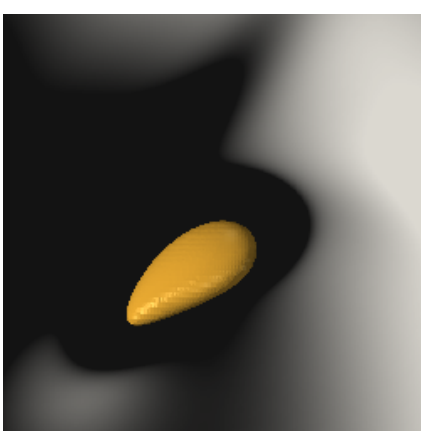

$t=201$

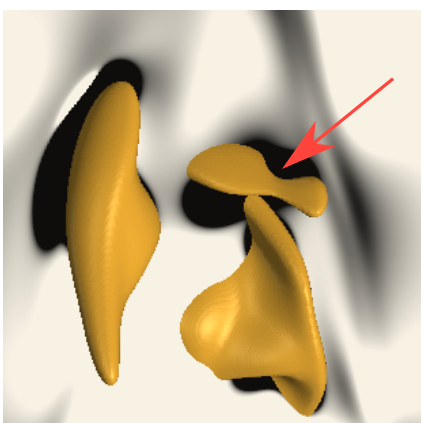

$$
t=202.3
$$

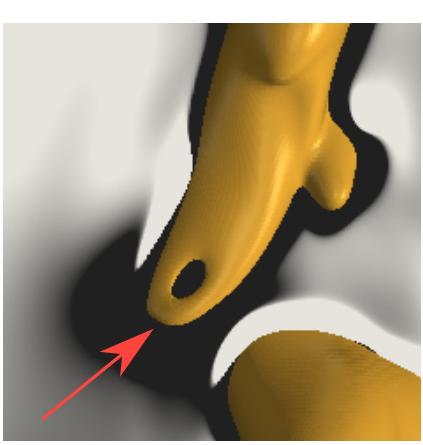

$t=201.1$

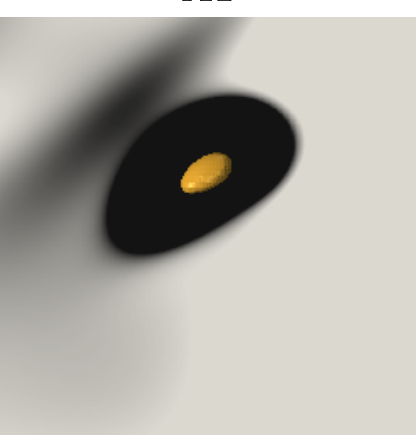

$$
t=201.5
$$

A2

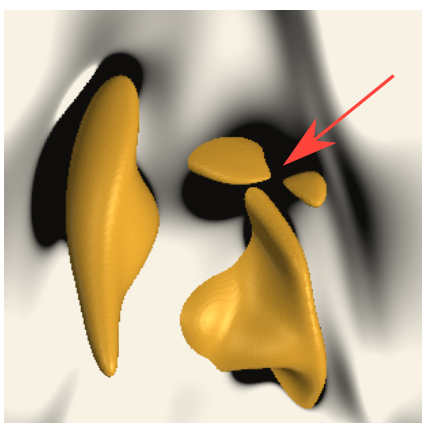

$$
t=202.4
$$

A3

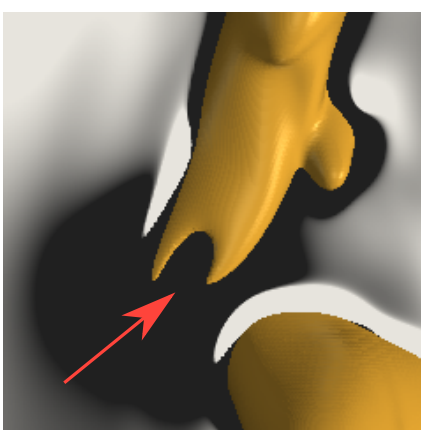

$$
t=201.2
$$

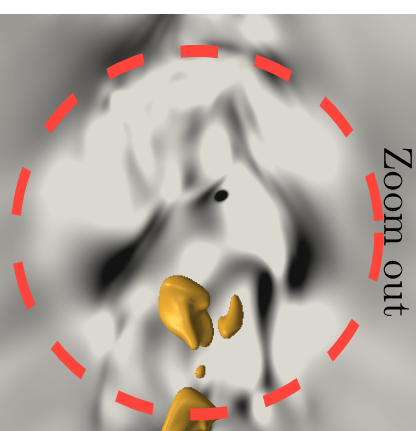

$t=202.2$

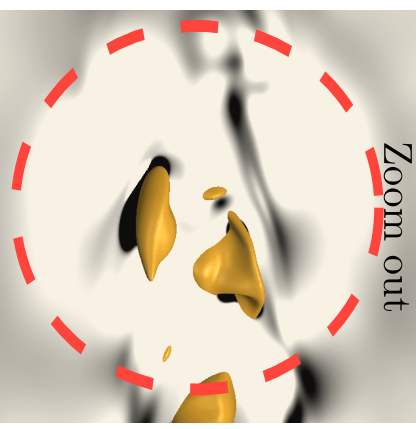

$t=203.5$

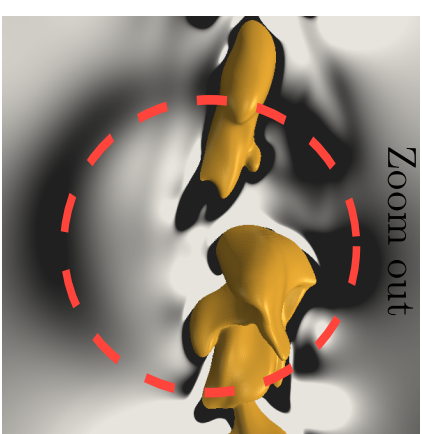

$$
t=201.8
$$

Figure 4.9: Instantaneous iso-surface with $Y_{R}=0.122$ superimposed on the dilatation field for the three labelled events A1, A2 and A3 in figure 4.7. The dashed circle shows the monopolar sound wave generated by each annihilation event. 
thirty two virtual microphones that are uniformly-distributed at $r=6 D$, as shown in figure 4.11. To reduce the so-called 'spectral leakage', a modified Hanning window Lockard, 2000] with 50\% overlap [Bui et al., 2008] was used to perform the fast Fourier transform (FFT) at each probe.

The averaged spectrum for both cases is shown in figure 4.12, Similar to earlier experimental studies (e.g Rajaram \& Lieuwen 2009]), the produced sound is broadband in our study. In addition, the magnitude of the noise radiated by the stoichiometric flame is greater than that of the lean flame, in particular further downstream of the jet inlet. Figure 4.13 confirms this by depicting the RMS acoustic pressure $p_{\text {rms }}^{\prime}$ for these 32 probes at each $x / D$ location. It is observed that close to the inlet both cases have almost the same $p_{\text {rms }}^{\prime}$, with a noticeable difference further downstream as the noise radiating from the inflow becomes weaker.

Using the values calculated for the flame lengths, the peak Strouhal numbers for the stoichiometric and lean flames (average of three $x / D$ locations) are 1.1 and 1.4 , respectively, which fall within the range found experimentally by Rajaram \& Lieuwen 2009.

However, Rajaram \& Lieuwen 2009] also observed that the high frequency spectra rolls off at $42-64$ (dB/decade) across $S t_{L_{\text {flame }}} \sim 1-100$ for several different flames with $R e_{D}>10^{4}$. This is not observed in the present study. There are several potential reasons for this observed difference. Those expected to be of particular significance are the two studies' differing inflow turbulence, differing forms of flame stabilisation, and the present study's lower jet Reynolds number of $R e_{D} \sim 5000$. It should be also noted that this present study did not intend to replicate the experiments investigated by Rajaram \& Lieuwen [2009], which are beyond current computational limits. 
(a)
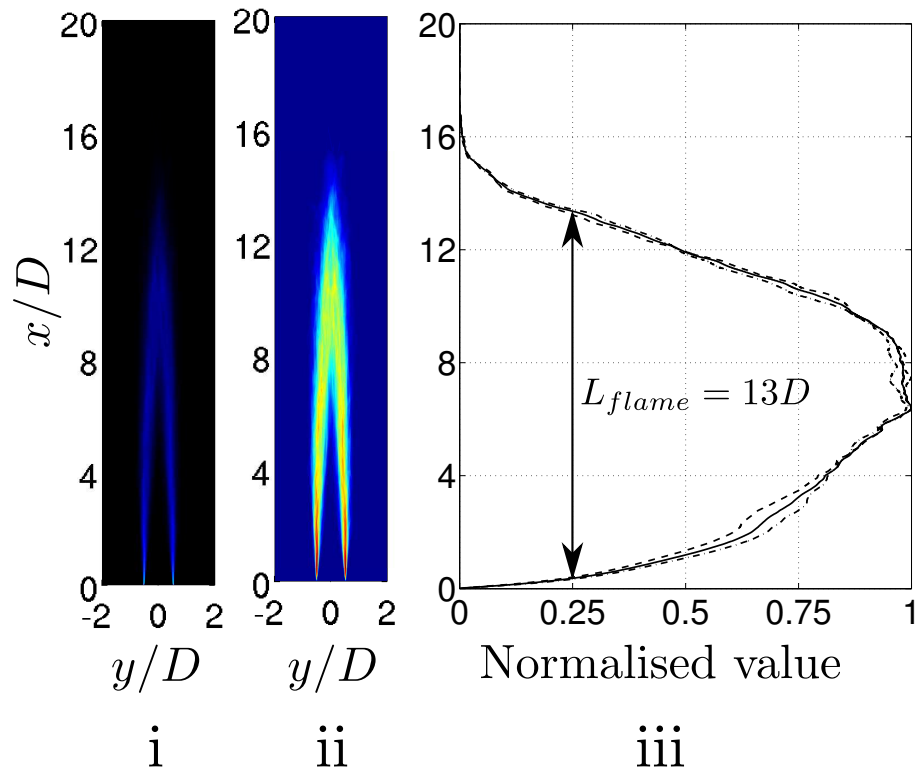

iii

(b)
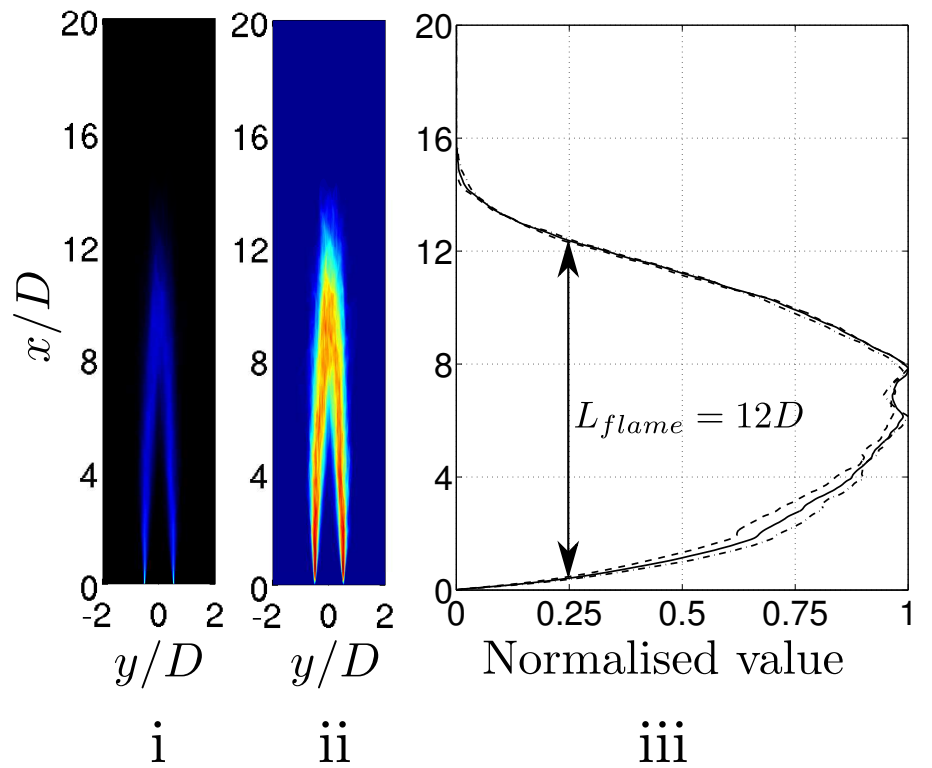

Figure 4.10: (i) Time-averaged reaction rate; (ii) variance of reaction rate; (iii) horizontally integrated variance field normalised by its maximum value (dashed: $\mathrm{x}-\mathrm{y}$ plane, dash-dot: $\mathrm{x}-\mathrm{z}$ plane and solid: the average of these planes) for (a) $\phi=1.0$ and (b) $\phi=0.7$. 

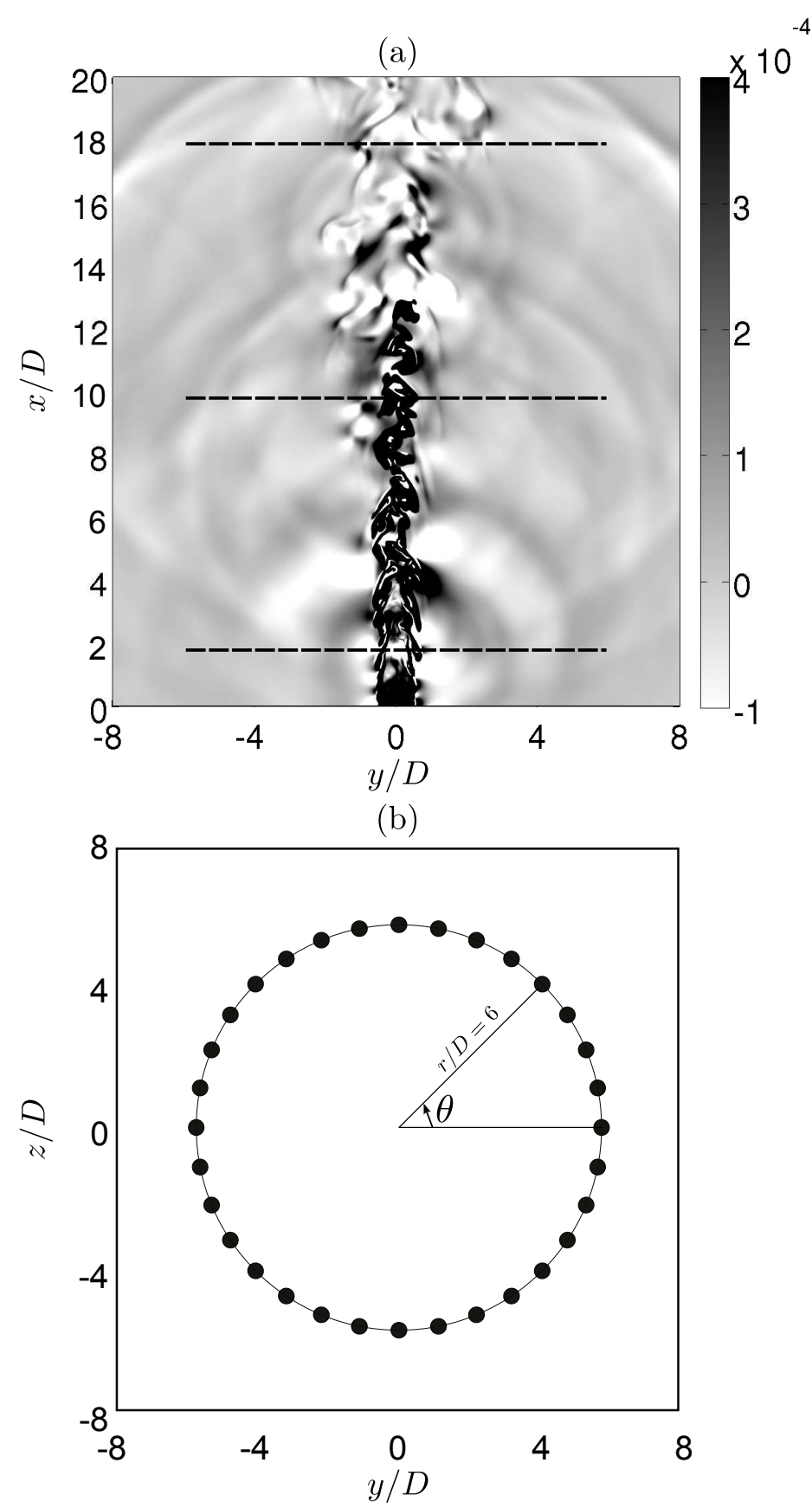

Figure 4.11: Thirty two virtual probes located at three different axial locations $x / D=2,10$ and 18 (a), distributed uniformly on a circle with $r / D=6$ at each particular $x / D$ location (b). 
(a)

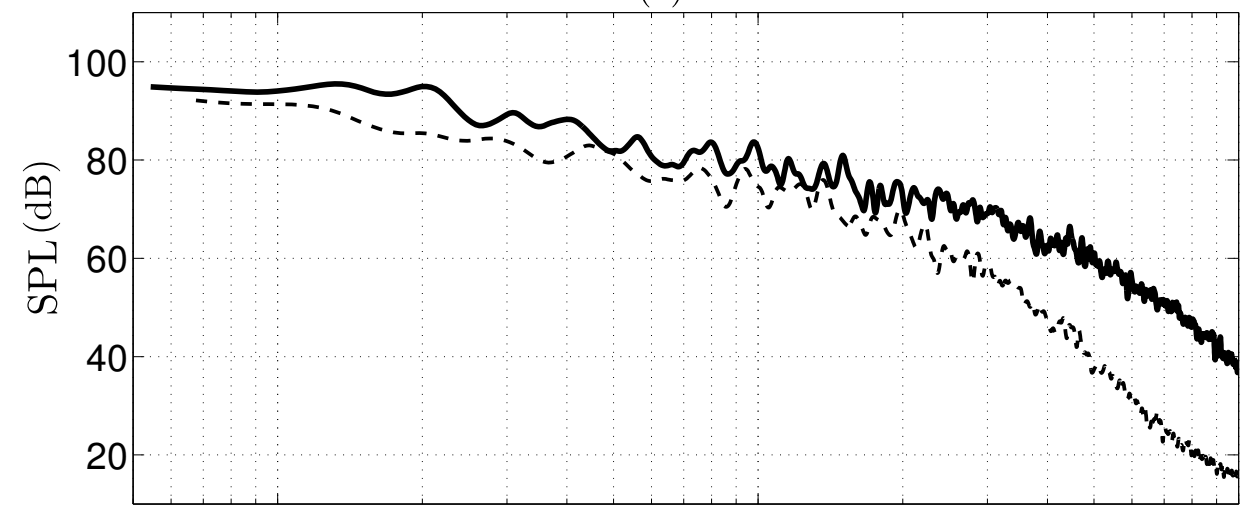

(b)

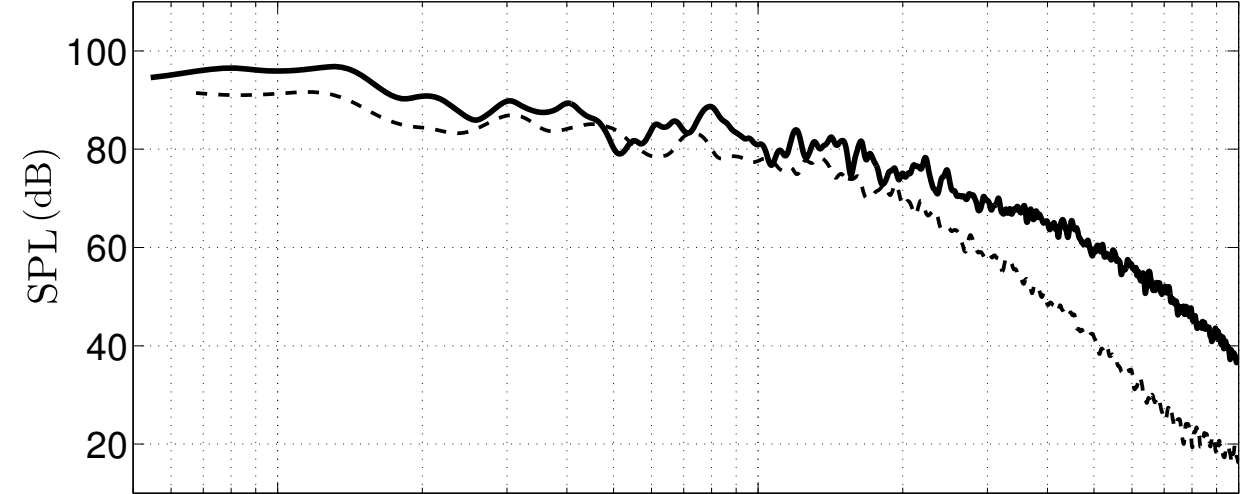

(c)

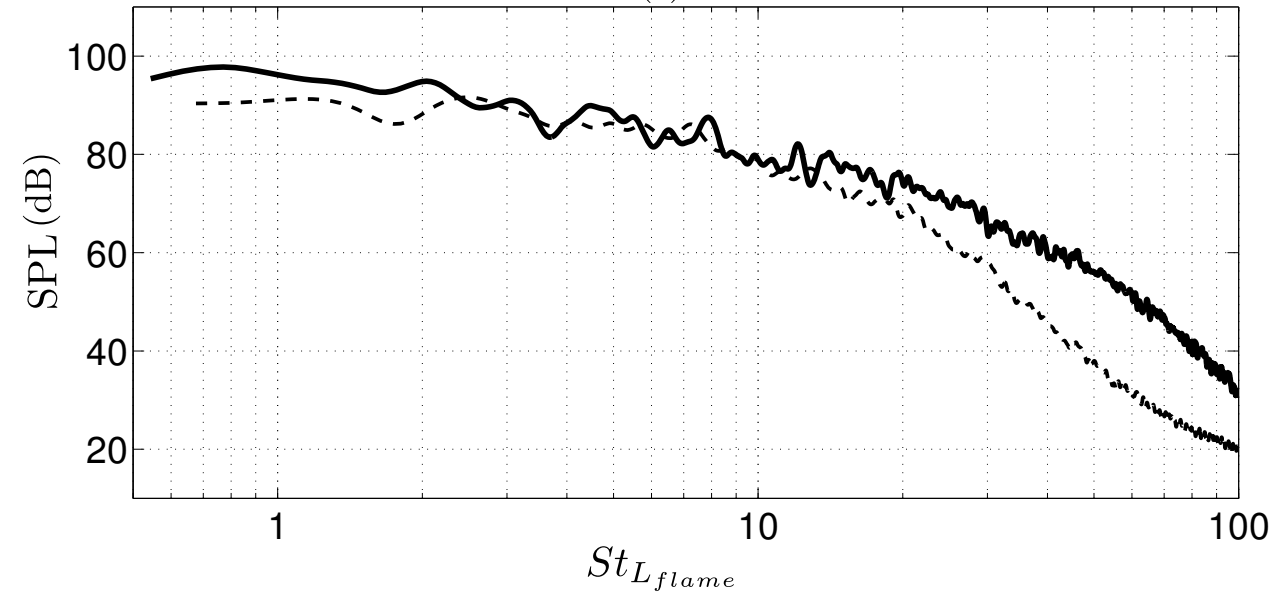

Figure 4.12: Noise spectrum for $\phi=1.0$ (solid) and $\phi=0.7$ (dashed) averaged over 32 equally spaced probes at $x / D$ : (a) 18 , (b) 10 and (c) 2. The horizontal axis shows Strouhal number based on flame length $\left(S t_{L_{\text {flame }}}=f L_{\text {flame }} / U_{a v}\right)$. The vertical axis shows the sound pressure level (SPL) in $\mathrm{dB}$. 
(a) $x / D=18$

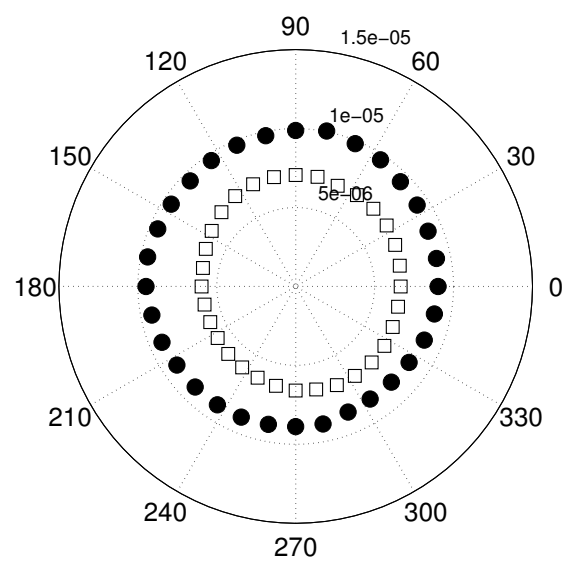

(b) $x / D=10$

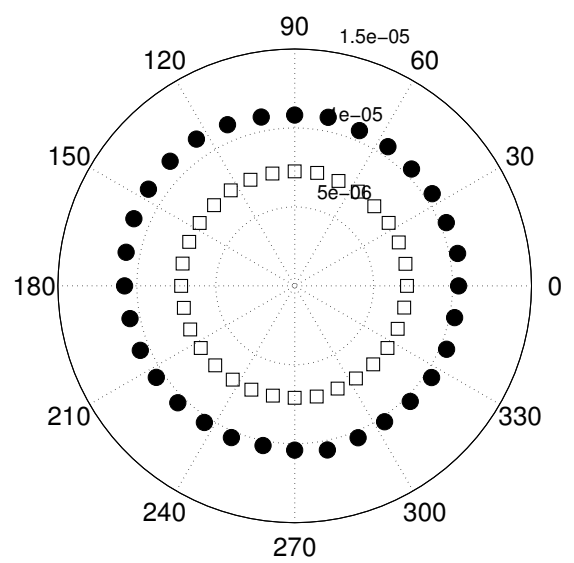

(c) $x / D=2$

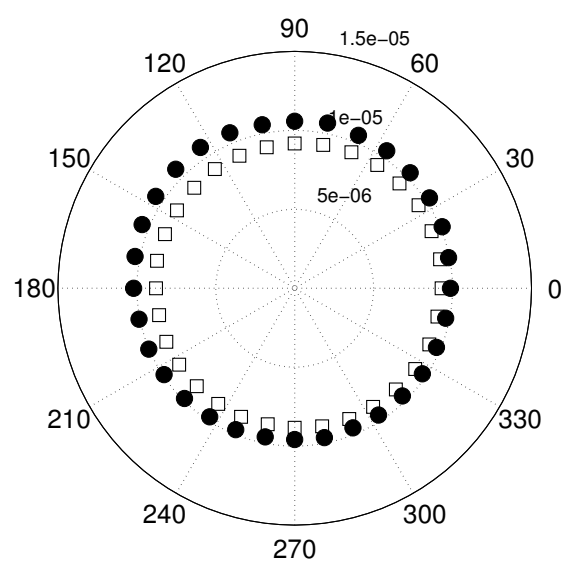

Figure 4.13: RMS of the acoustic pressure $\left(p_{\text {rms }}^{\prime}\right)$ for $\phi=1.0(\bullet)$ and $\phi=0.7$ $(\square)$ considering thirty two virtual probes at $r / D=6$ and three different axial locations $x / D$ : (a) 18 , (b) 10 and (c) 2 . 


\subsection{Conclusions}

This chapter presented Direct Numerical Simulations (DNS) of sound generation by turbulent premixed flames. Two cases of methane/air flames with different equivalence ratios ( $\phi=0.7$ and 1$)$ were considered. A single-step chemistry model was used to reduce the computational cost. Care was taken to fully resolve both the near and far fields and avoid spurious noise reflections at the outflow boundaries.

Several significant features of these two simulated flames were noted. First, the acoustic fields of both the stoichiometric and lean flames exhibited monopolar features that originated within the flame, with the stoichiometric flame being significantly louder than the lean flame. These observations are consistent with several experimental studies of turbulent jet flames in the literature. Further, socalled 'flame annihilation' events were observed to be a source of these monopolar, acoustic features, which was consistent with prior works on laminar premixed flames.

Three types of flame annihilation events were observed:

- 'island burn-out'

This event involves consumption of a pocket of unburned gases, particularly in the downstream region near the flame tip.

- 'pinch-off'

This events occurs where a pocket of unburned gases is locally detached from the flame surface.

- 'tunnel formation'

This event takes place when flame-to-flame interaction results in the formation of a tunnel of burned gas. The local flame area is then lost due to cusp retraction associated with another 'pinch-off' event.

The acoustic spectra of both flames were also studied. These were found to be broadband in nature, with a frequency at the peak spectral amplitude agreeing with that observed experimentally by Rajaram \& Lieuwen 2009. 


\section{Chapter 5}

\section{Sound Generation by Annihilation Events in Turbulent Premixed Flames}

\subsection{Introduction}

It has already been shown qualitatively that flame annihilation events play a noticeable role in the sound generated by a turbulent premixed flame. However, a more detailed analysis is required to quantify the contribution of the flame annihilation events to the overall radiated sound. This chapter therefore undertakes such analysis. First, a marker for identification of the observed flame annihilation events is defined. A simple model that relates these events to the far-field sound is then proposed. Finally, the DNS and the model predictions are compared. This comparison sheds light on the significance of flame annihilation events in sound generation by turbulent premixed flames.

\subsection{Identification of Flame Annihilation}

It has been argued in the previous chapter that flame annihilation events play a significant role in the noise generated by a turbulent premixed flame. A means of identifying these events should therefore be defined. It is known that flames accel- 
erate prior to flame annihilation (e.g. Echekki et al. [1996]; Sun \& Law [1998]). The impact of this acceleration on the produced sound was also discussed by Talei et al. 2011. To capture this acceleration, the density-weighted displacement speed of the flame relative to the local gas velocity [Echekki \& Chen, 1999] is therefore considered in non-dimensional form,

$$
S_{d}^{*} \equiv \frac{\rho S_{d}}{\rho_{u}}=-\frac{\dot{\omega}_{R}}{\rho_{u}\left|\nabla Y_{R}\right|}-\frac{1}{S c R e} \frac{\mathbf{n} \cdot \nabla\left(\mu\left|\nabla Y_{R}\right|\right)}{\rho_{u}\left|\nabla Y_{R}\right|}-\frac{\mu}{\operatorname{ScRe} \rho_{u}}(\nabla . \mathbf{n})
$$

The density-weighted form of the flame displacement is used since this minimises the effect of thermal expansion on the displacement speed. Here, $(\nabla \cdot \mathbf{n})$ is the flame curvature where $\mathbf{n}$ is the unit normal vector of the iso-contour of $Y_{R}$ directed towards the reactant,

$$
\mathbf{n}=\frac{\nabla Y_{R}}{\left|\nabla Y_{R}\right|}
$$

The flame curvature is taken to be negative (positive) when the flame is curved towards the fresh reactant (burned mixture). Note that equation 5.1 shows that the flame displacement speed is obtained by the relative contributions of the reaction rate, the normal diffusion and the flame curvature.

Figure 5.1 illustrates the iso-surface of the progress variable $Y_{R}=0.122$ corresponding to the three distinct flame annihilation events A1 to A3 in figure 4.7. These iso-surfaces are now coloured with the normalised density weighted flame displacement speed $\left(S_{d}^{*} / S_{L}\right)$. There is a large increase in the magnitude of $S_{d}^{*} / S_{L}$ during the annihilation events. Thus, $S_{d}^{*} / S_{L}>10$ seems to be a reasonable condition for identifying annihilation events.

Figure 5.2 therefore shows scatter plots of grid nodes that satisfy this proposed identifier of flame annihilation, i.e. points with $Y_{R}=0.122$ for $\phi=1.0\left(Y_{R}=\right.$ 0.156 for $\phi=0.7)$ and $S_{d}^{*} / S_{L}>10$. These scatter plots were generated using the same time period, which was the flow through time of the stoichiometric case detailed in table 3.1. Events throughout the stoichiometric and lean flames are evident and common. Perhaps surprisingly, the lean flame shows more of these events even though figure 4.7 and figure 4.12 showed that it was the quieter flame. However, this is not necessarily a contradiction, particularly since premixed flame noise is known to scale with the laminar flame speed [Smith \& Kilham, 1963; 
A1

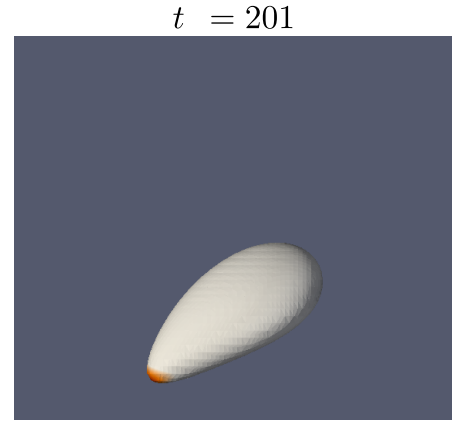

$t=201.5$

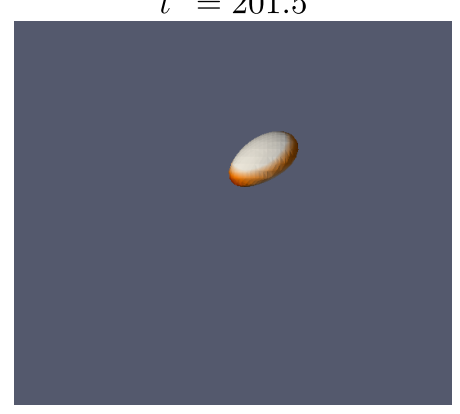

$\mathrm{A} 2$

$t=202.2$

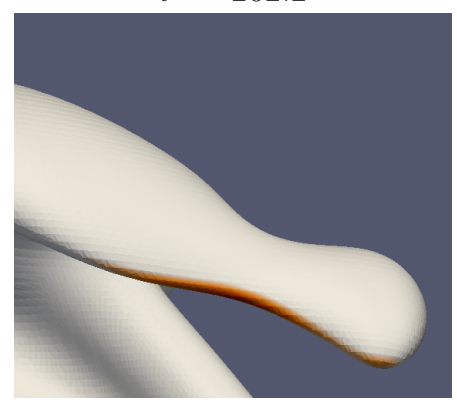

$t=202.3$

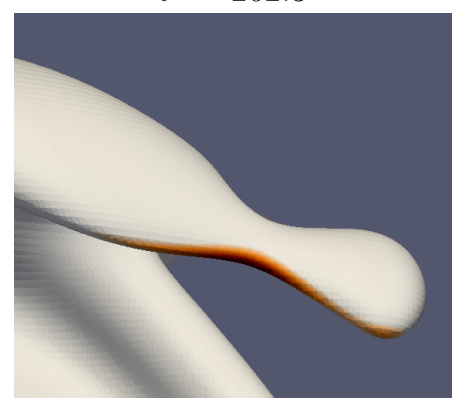

A3

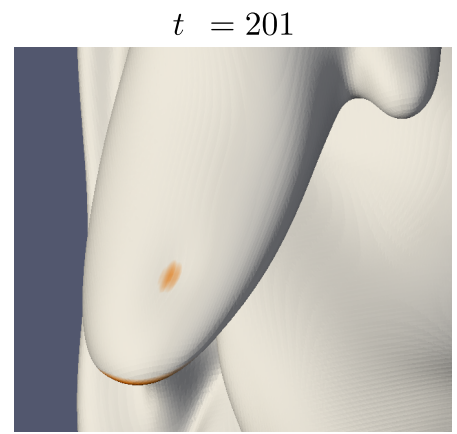

10

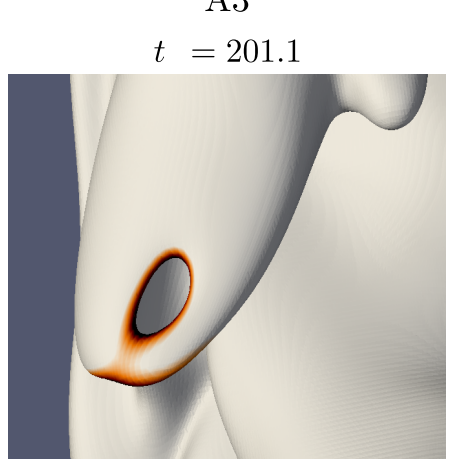

20

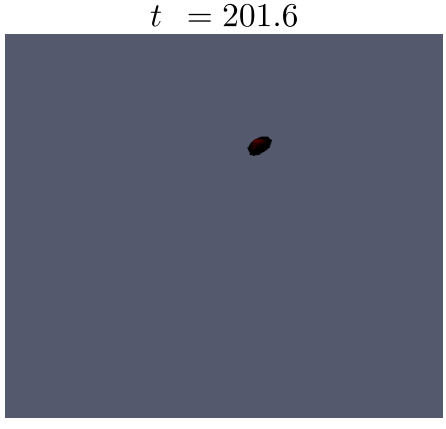

$t=202.4$
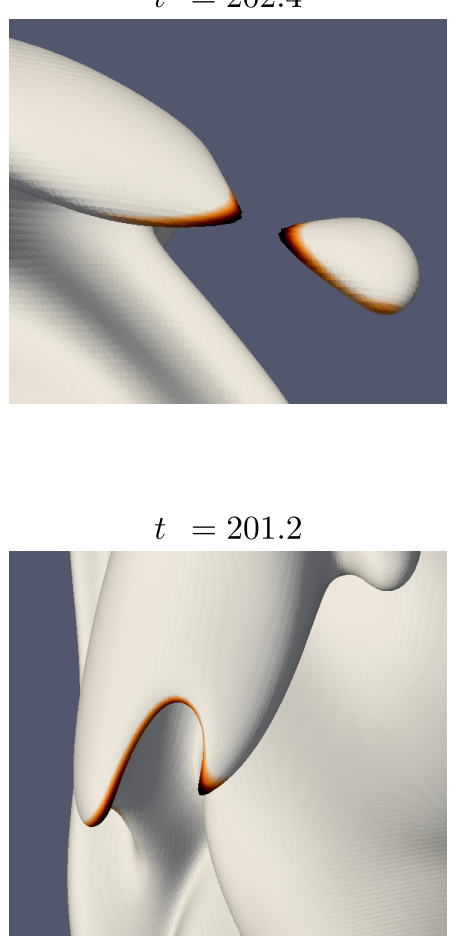

30

Figure 5.1: The time evolution of the three flame annihilation events labelled in figure 4.7 and their corresponding normalised density weighted flame displacement speed $\left(S_{d}^{*} / S_{L}\right)$ fields mapped on the flame surface.

Strahle \& Shivashankara, 1975, Talei et al., 2011]. It is therefore now considered 
(a)

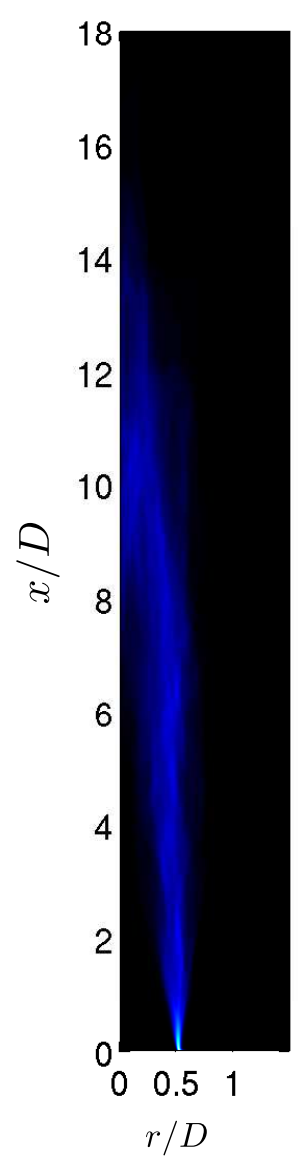

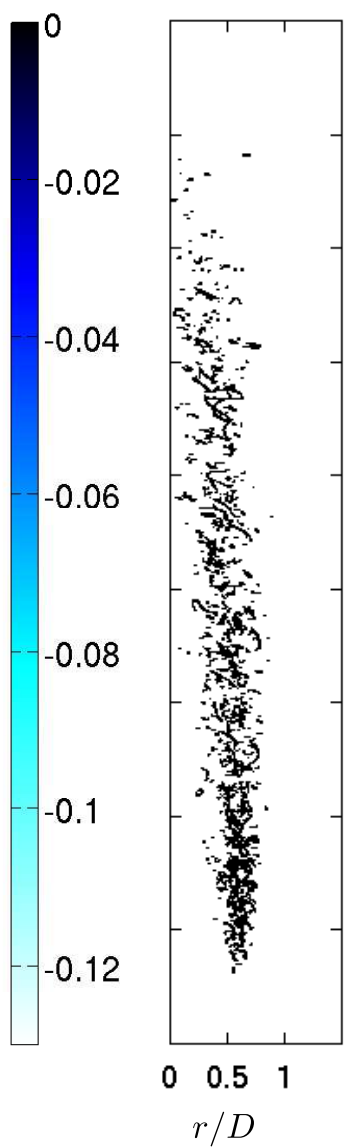

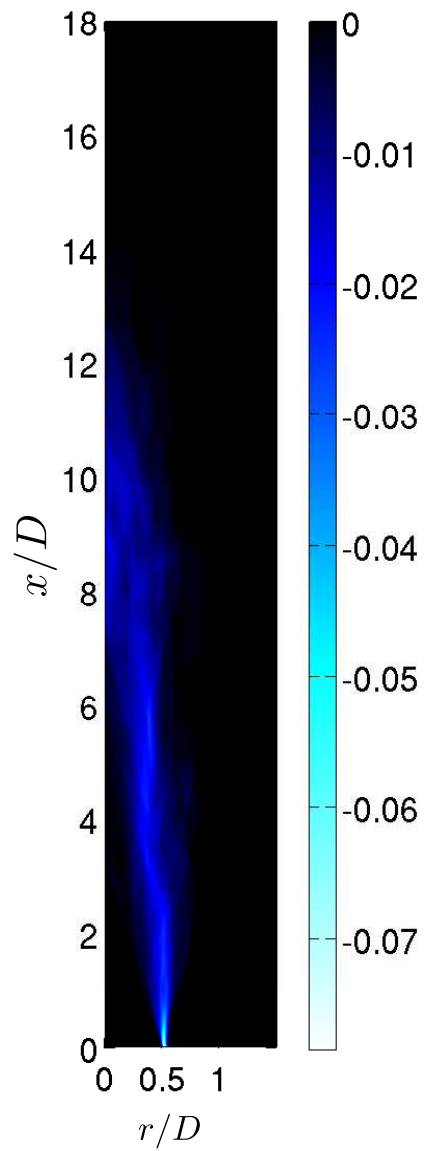

(b)

Figure 5.2: Average reaction rate and scatter plot of nodes with $S_{d}^{*} / S_{L}>10$ on an iso-surface with a) $Y_{R}=0.122$ for $\phi=1.0$ and b) $Y_{R}=0.156$ for $\phi=0.7$. The stoichiometric flow through time resulting from table 3.1 was used to produce plots.

how identification of these events can be used in a simple model of the radiated sound. 


\subsection{A Simple Model of Sound Generation by Flame Annihilation}

A simple model of sound generation by these annihilation events is now considered. This model is consistent with the approach of Smith \& Kilham [1963], and considers the flame to be a distribution of annihilation events, each of which radiates monopolar sound to the far-field. This simple model is therefore only a first step in assessing the contribution of annihilation events to the overall sound produced by turbulent premixed flames, and is not intended to be a complete model of sound production. A comprehensive description of all different types of annihilation events is not considered, and other acoustic sources are neglected.

\subsubsection{Laminar Flame Annihilation}

Spherically symmetric annihilation events are simulated first. The flame parameters are the same as those used in the turbulent flames for both stoichiometric and lean conditions. After initialising the flow field, the spherical flames start to propagate from their initial position at $r_{0} / \delta_{t h}=7$, where $r_{0}$ is the distance from the origin.

Figure 5.3 shows $S_{d}^{*} / S_{L}$ as a function of $r_{0} / \delta_{t h}$ for both cases. It is observed that $S_{d}^{*} / S_{L}$ has a value of about unity when the flame is at $r_{0} / \delta_{t h} \approx 2$. After that, $S_{d}^{*} / S_{L}$ starts to increase, reaching very large values during annihilation, as several other studies have found (e.g. Echekki et al. 1996; Sun \& Law 1998).

Figure 5.4 shows a comparison of the sound produced by both annihilation events at $r / \delta_{t h}=28$, where $r$ is the distance between the receiver and the centre of the flame sphere. The annihilation event corresponding to the lean flame is much quieter than that of the stoichiometric flame. This is consistent with Talei et al. 2011], who found that the sound produced for a spherically symmetric annihilation event is proportional to $S_{L}^{2}$. This can also likely contribute to the lower level of noise produced by the turbulent lean flame.

\subsubsection{Theory}

Talei et al. 2012a] used the theory of Dowling [1992], which in turn extended 


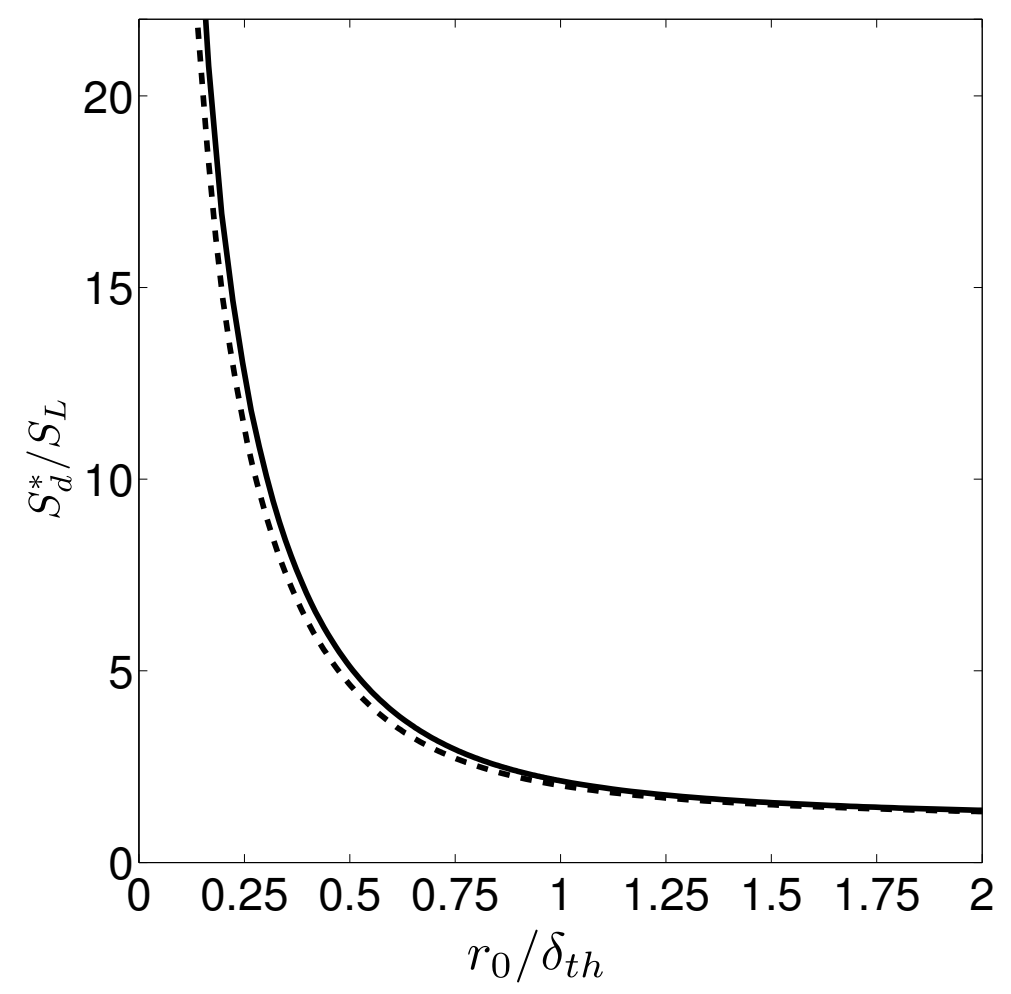

Figure 5.3: Normalised density weighted flame displacement speed $\left(S_{d}^{*} / S_{L}\right)$ as a function of normalised flame radius $\left(r_{0} / \delta_{t h}\right)$ for a spherically symmetric annihilation event for $\phi=1.0$ (solid) and $\phi=0.7$ (dashed).

Lighthills equation Lighthill, 1952, 1954 to combusting flows. By considering the fluctuations in heat release as the only source term, modelling the reaction rate as a delta-function and assuming a constant consumption speed, Talei et al. $2012 a$ showed that the general solution of Lighthill's equation for a spherically symmetric annihilation event is

$$
p^{\prime}(r, t)=-2 \rho_{u} S_{L}\left(1-\frac{T_{u}}{T_{b}}\right) \frac{r_{0}\left(t-r / c_{b}\right)}{r} V_{f}\left(t-r / c_{b}\right) H\left(t-r / c_{b}\right),
$$

where $p^{\prime}$ is the fluctuating part of the pressure, $c_{b}$ is the burned sonic speed, $H$ is the Heaviside function and $V_{f}$ is the magnitude of flame propagation velocity $\mathbf{V}_{f}$ projected onto the flame normal $\mathbf{n}$,

$$
V_{f} \equiv \mathbf{V}_{f} \cdot \mathbf{n}=\mathbf{u} \cdot \mathbf{n}+S_{d} .
$$


Figure 5.4 compares $p^{\prime}$ from DNS and equation 5.3 for spherical annihilation events. The over-prediction of the peak pressure is due to the assumption of constant consumption speed, particularly at the later stages of the annihilation event Talei et al., 2012a]. The step at the peak acoustic pressure in the theory is because the flame is represented as a delta function.

\subsubsection{Model Development for Turbulent Premixed Flames}

\subsubsection{Model Description}

Extension of equation 5.3 for different types of annihilation events could involve an extensive numerical and theoretical study to construct a model which incorporates the many features of different types of annihilation events. However, as the first step, all annihilation events are assumed to be spherically symmetric in a deliberately simple model.

The iso-surface of the progress variable corresponding to the maximum reaction rate $\left(Y_{R}=0.122\right.$ and 0.156 for $\phi=1.0$ and $\phi=0.7$ respectively) are then conditioned using $S_{d}^{*} / S_{L} \geq n$ as a marker. Figure 5.5 shows the effect of different values of $n$ on the noise generated by a spherically symmetric annihilation event using a modified form of equation 5.3 ,

$p^{\prime}(r, t)=-2 \rho_{u} S_{L}\left(1-\frac{T_{u}}{T_{b}}\right) \frac{r_{0}\left(t-r / c_{b}\right)}{r} V_{f}\left(t-r / c_{b}\right) H\left(t-r / c_{b}\right) H\left(\frac{S_{d}^{*}\left(t-r / c_{b}\right)}{S_{L}}-n\right)$.

It can be seen that when $n=2.5$ is chosen, the whole annihilation is captured whereas for $n=20$, only the final stage of annihilation is recovered. Equation 5.5 requires the information at $t-r / c_{b}$ for each annihilation event. This instant might not coincide with the instants at which the DNS data are collected. We therefore used a time shift $r / c_{b}$ to obtain the pressure fluctuations at a later time as follows:

$$
p^{\prime}\left(r, \tau+r / c_{b}\right)=-2 \rho_{u} S_{L}\left(1-\frac{T_{u}}{T_{b}}\right) \frac{r_{0}(\tau)}{r} V_{f}(\tau) H\left(\frac{S_{d}^{*}(\tau)}{S_{L}}-n\right),
$$

where $\tau=t-r / c_{b}$ and $r_{0}$ is determined from figure 5.3 at a given $S_{d}^{*} / S_{L}$. 


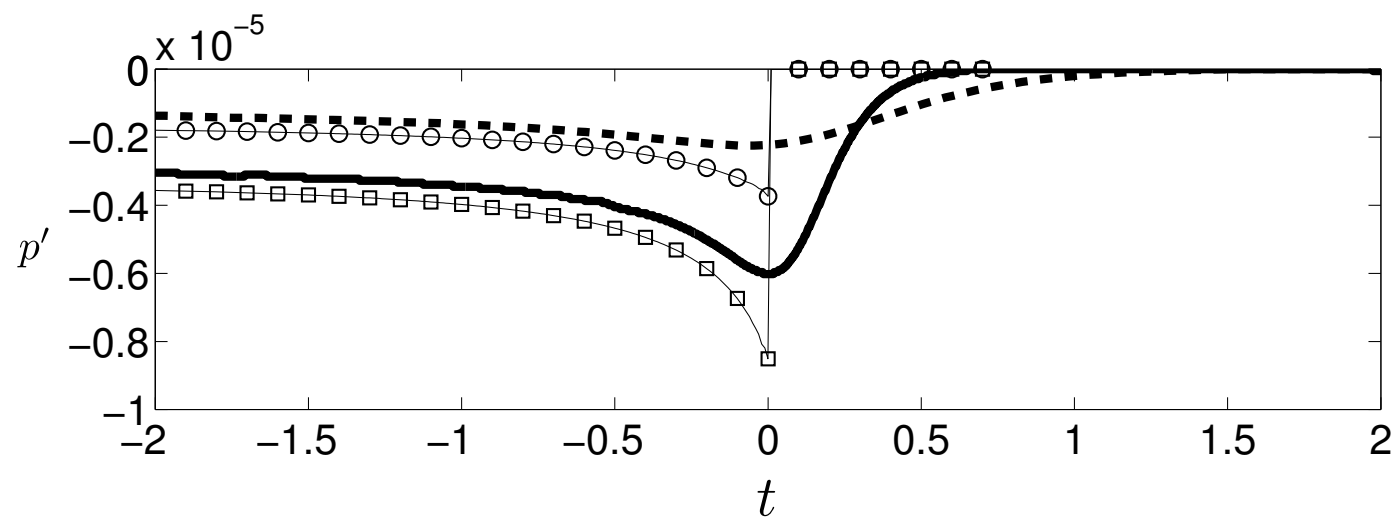

Figure 5.4: Time history of sound generated by a spherically symmetric annihilation event at $r / \delta_{t h}=28$ from DNS for $\phi=1.0$ (solid) and $\phi=0.7$ (dashed) and by equation 5.3 for $\phi=1.0$ (square) and $\phi=0.7$ (circle).

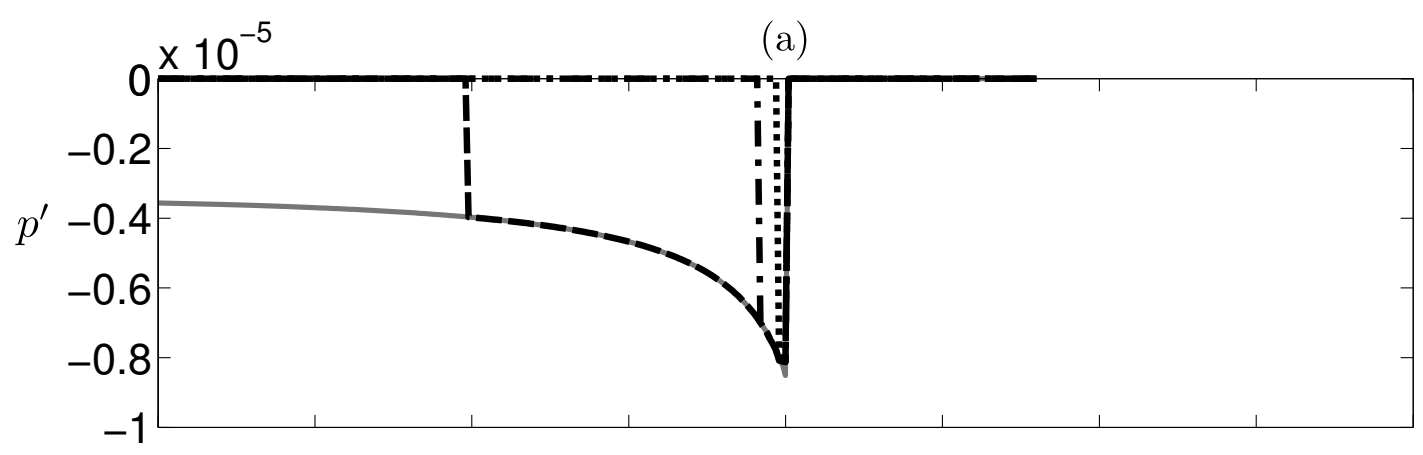

(b)

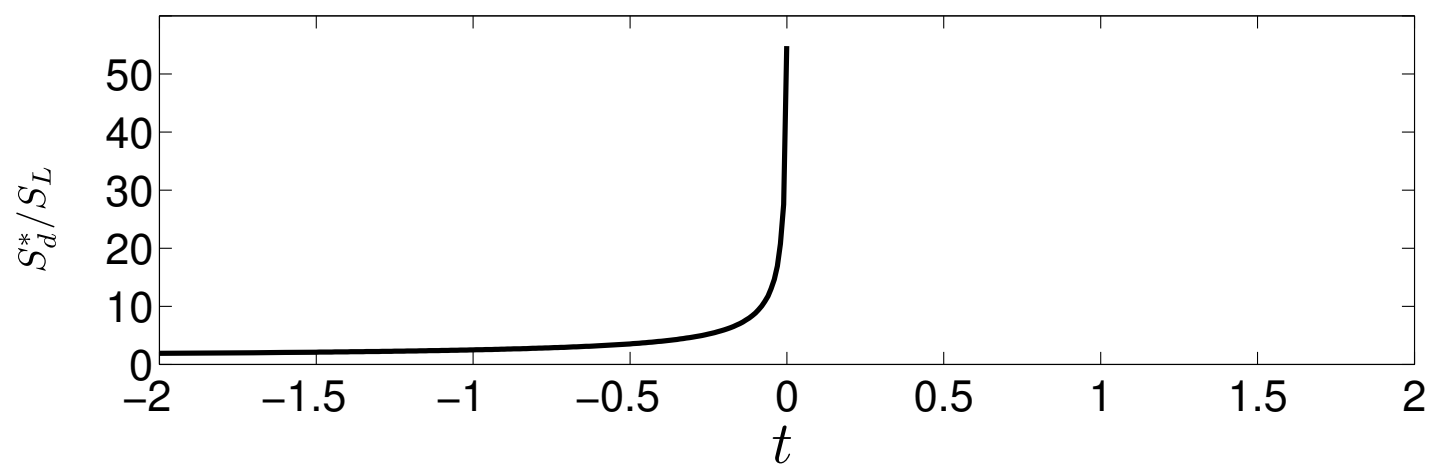

Figure 5.5: (a) Comparison of the sound generated by a spherically symmetric annihilation event at $r / \delta_{t h}=28$ using equation 5.3 (solid) and the modified theory (equation 5.5) with $n=2.5$ (dashed), 10 (dash-dot) and 20 (dotted). (b) The corresponding time history of $S_{d}^{*} / S_{L}$ for this event. 


\subsubsection{Algorithm}

There are four steps in our modelling algorithm:

1. Divide the domain into cubes

2. Identify flame annihilation events

3. Represent the flame with a number of spherically symmetric annihilation events

4. Calculate the total sound produced

Figure 5.3 shows that having information from the instant at which $r_{0}=\delta_{t h}$ is sufficient to represent the annihilation event. Note that $r_{0}=\delta_{t h}$ corresponds to $S_{d}^{*} / S_{L} \approx 2.5$ which is the lowest threshold for $S_{d}^{*} / S_{L}$ considered in this study. It is therefore assumed that each cube in the domain has a size of $2 \delta_{t h}$ in which only one spherically symmetric annihilation event can occur at any time. The entire domain that features the flame is therefore divided into these uniformly distributed cubes (figure 5.6a).

For step 2, the points on the iso-surfaces of $Y_{R}$ (0.122 and 0.156 for the stoichiometric and the lean cases, respectively) in each cube with $S_{d}^{*} / S_{L} \geq n$ and $V_{f}>0$ are identified at each instant. Figure $5.6 \mathrm{~b}$ shows examples of the identified points for different types of annihilation events.

For step 3, the cluster of points in each cube is now replaced with one point at the centroid of the cluster. This point is considered as one spherically symmetric annihilation event. Figure 5.6c shows how the flame pinch-off event labelled as B1 is represented by one spherically symmetric annihilation. Another flame pinch-off event labelled as B2 is considered as two events since it is split into two cubes. Although this event is very similar to that labelled as B1, it will be modelled as a sound source of twice the strength. This is an unavoidable and undesirable feature of this algorithm, with some over-prediction of the sound expected to result. Finally, the event labelled as B3 shows a long, curved flame 
edge distributed over several cubes. Once again, this will potentially contribute to over-prediction of the sound.

Step 4 then calculates the overall sound produced by calculating the noise generated by each annihilation event. First, a number of time bins (with the bin size equal to the sampling size of the DNS data) are considered and the result of equation 5.6 for each representative point source is stored in its associated time bin. Then, $p^{\prime}$ in the far field resulting from the sound produced by all points is calculated by summing the stored values in each time bin.

\subsection{Comparison of the Simple Model with DNS}

Here, the present theory with the algorithm described earlier is used to predict the far-field noise and the results are compared with the DNS data. Figure $5.7 \mathrm{a}$ depicts the time history of the acoustic pressure $p^{\prime}$ at $x=10 D$ and $r=6 D$ obtained from the DNS for the case with $\phi=1.0$. Figure $5.7 \mathrm{~b}$ and $\mathrm{c}$ show the corresponding model results for $n=10$ and 5, respectively. The modelled signals contain some high frequency noise which is due to the model representing the flame as discrete point sources that appear and disappear rapidly due to the conditioning on $S_{d}^{*} / S_{L}$. The correlation coefficient, $\rho=\operatorname{cov}(X, Y) /(\sigma(X) \sigma(Y))$, between the modelled signal and the DNS data is $36 \%$ for $n=10$ and $66 \%$ when $n=5$ is used. A comparison of figure $5.7 \mathrm{~b}, \mathrm{c}$ and a also shows that the magnitude of the pressure fluctuations is over-predicted by the model, as discussed in section 5.3.3.2. Clearly, the choice of threshold $n$ is important.

A range of $n$ from 2.5 to 20 is therefore considered. For high values of $n$, some of the annihilation events are not captured because of the finite time resolution of the recorded DNS data. On the other hand, choosing a low value of $n$ will include parts of the flame surface which are not annihilation events. This suggests that there will be an optimum value of $n$ in terms of correlation coefficient. Figure 5.8 supports this hypothesis by showing that $n=5$ and $n=12.5$ are optimal for the stoichiometric and lean cases, respectively. The optimal correlation is particularly strong for the stoichiometric flame, demonstrating the significant contribution of annihilation events to sound production. A more than two times higher value 
(a)

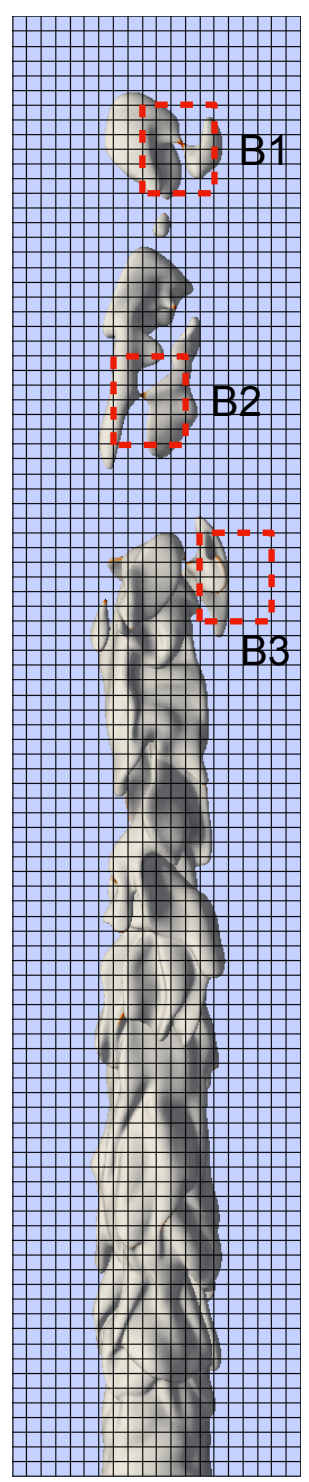

(b)

B1

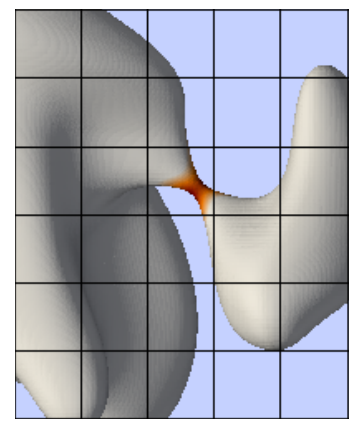

B2

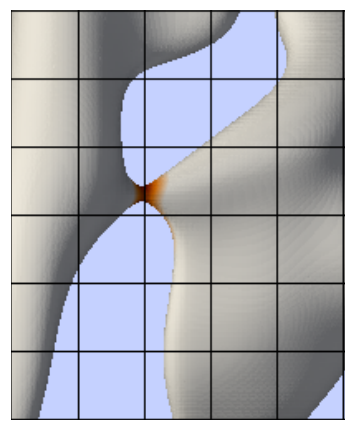

B3

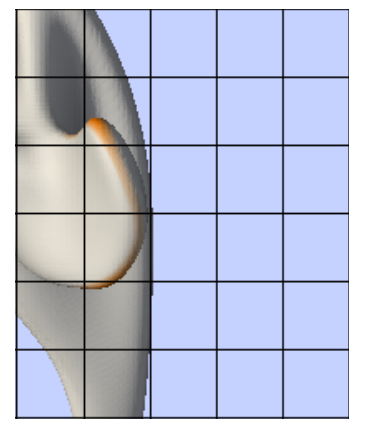

(c)
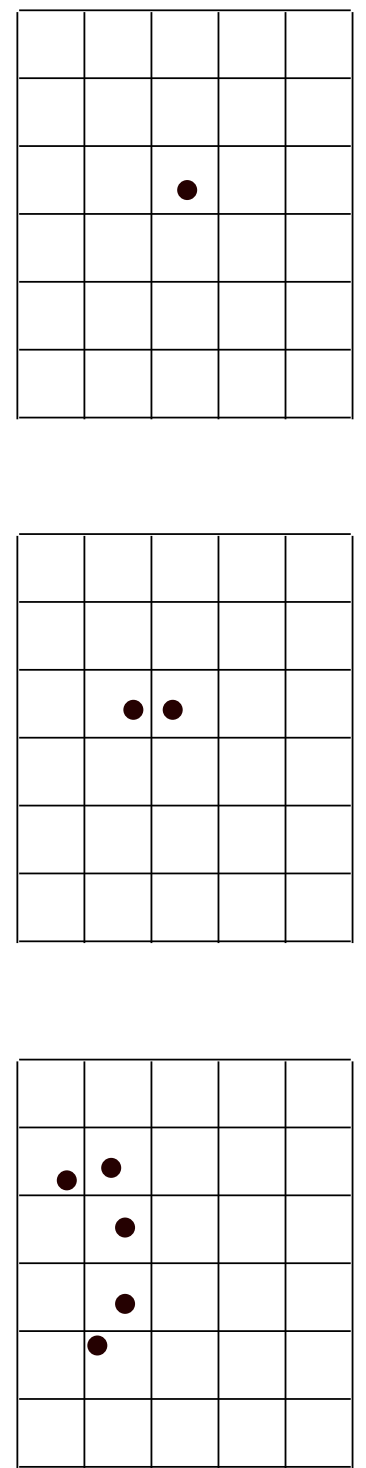

5 10 30

Figure 5.6: (a) One snapshot of the flame iso-surface for $\phi=1.0$ on a uniform mesh with a cube of edge length $\Delta=2 \delta_{t h}$. (b) The regions B1, B2 and B3 in (a) are zoomed in and coloured with the $S_{d}^{*} / S_{L}$ field. (c) Centroid of the cluster of the conditioned data as per section 5.3.3.2. 

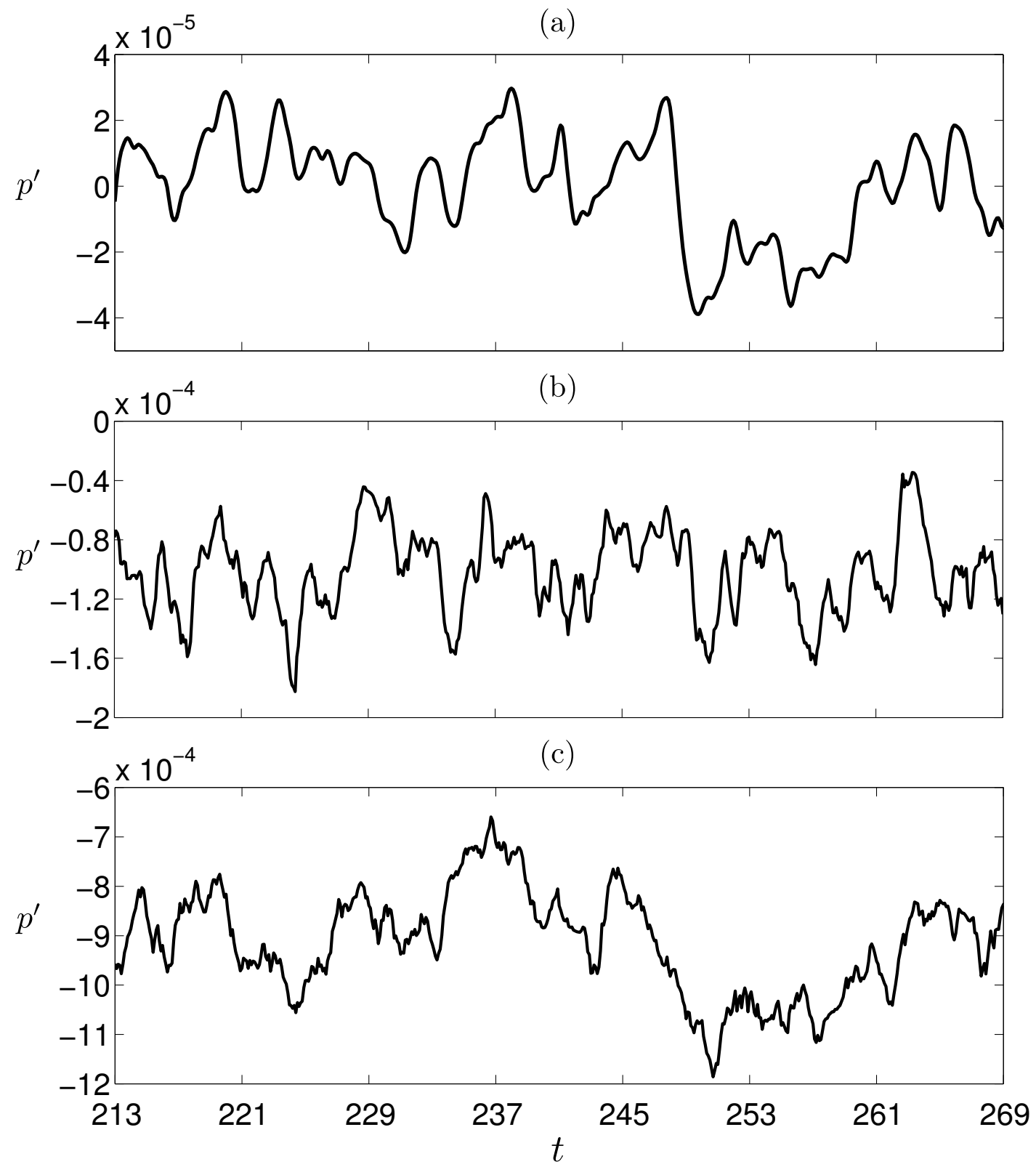

Figure 5.7: Time history of the far-field noise at $r=6 D$ and $x=10 D$ for $\phi=1.0$, obtained from (a) DNS, (b) model with $n=10$ and (c) model with $n=5$.

of $n$ for $\phi=0.7$ implies that the early stages of annihilation events in the lean flame are much weaker sources of noise compared with those of the stoichiometric flame.

Figure 5.9 shows this correlation coefficient over a $x-z$ plane located at 


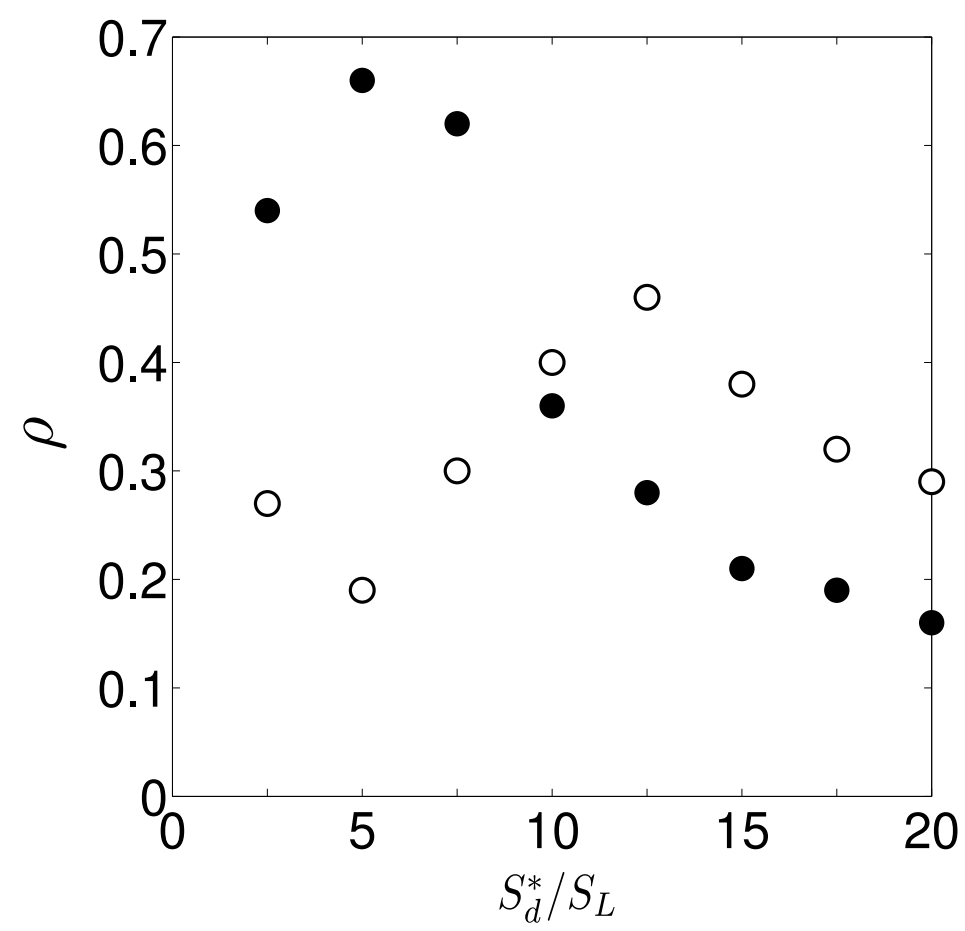

Figure 5.8: Correlation coefficient $\rho$ between the DNS far-field noise and that of the model at $r=6 D$ and $x=10 D$ as a function of $S_{d}^{*} / S_{L}$ for $\phi=1$ (solid circles) and $\phi=0.7$ (hollow circles).

$y / D=-6$. The optimum values of $n$ for both flames in figure 5.8 are used to calculate the correlation coefficient. While an almost uniform distribution is observed for the stoichiometric flame (figure 5.9 ), two distinct zones are found for the lean flame (figure $5.9 \mathrm{~b}$ ). This shows that annihilation events are a strong source of noise for the stoichiometric flame. There is, however, a competition between the sound produced by the annihilation events and other sources such as the inflow noise for the quieter, lean flame.

The degree of correlation between the DNS and the model in figure 5.9 is surprising given the model's simplicity. The model considers all sound sources to be spherically symmetric annihilations, even though other forms of annihilation were shown to exist and other sound sources are expected in combusting flows Dowling, 1992; Talei et al., 2014]. Further, all sound is modelled as travelling radially from these sources at the burnt gas velocity, ignoring refraction and sound propagation at lower velocity, as should occur for a source on one side of the flame 
(a)

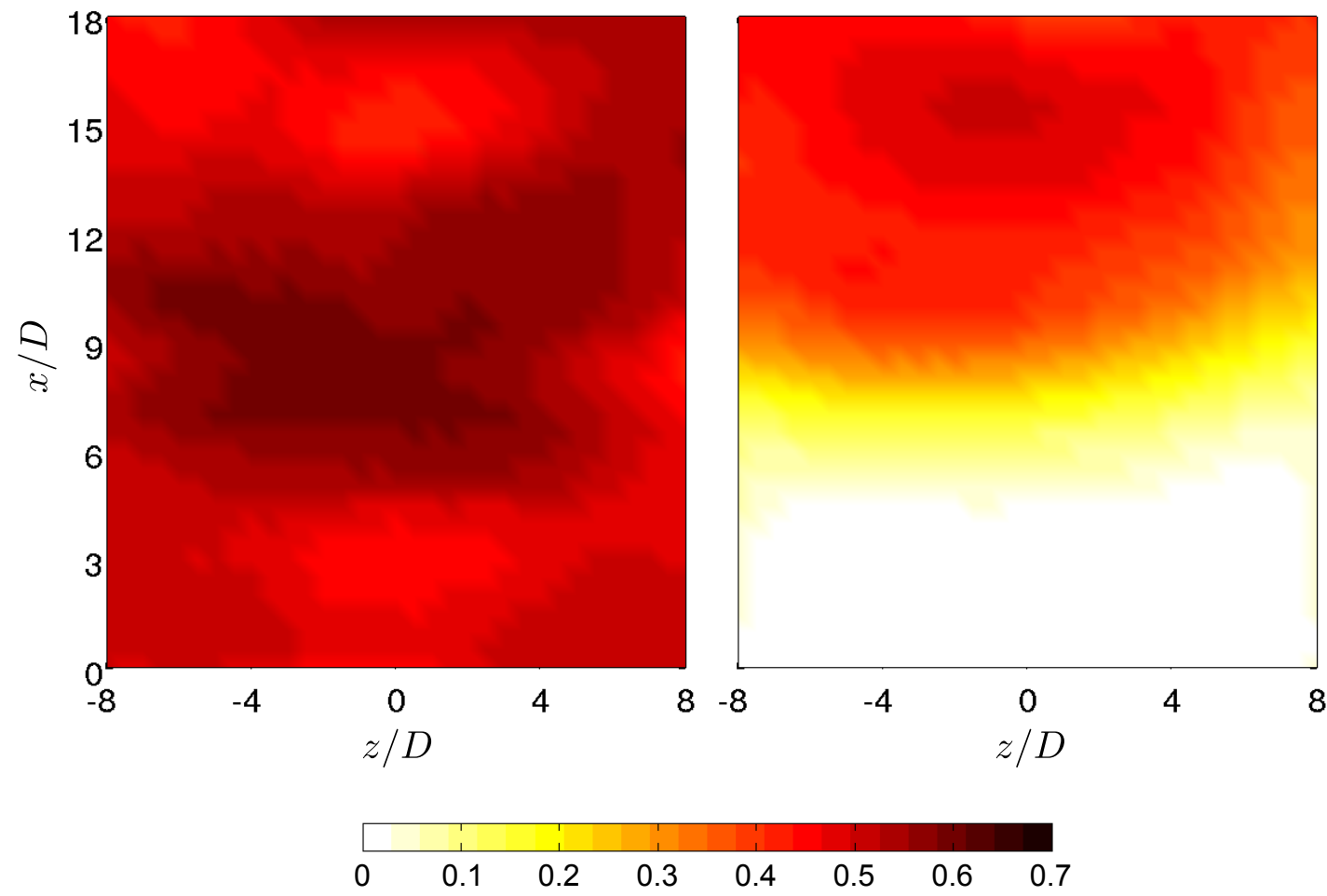

Figure 5.9: Distribution of the correlation coefficient $\rho$ between the DNS and the model calculated over a $x-z$ plane located at $y / D=-6$ for (a) $\phi=1.0$ with $n=5$ and (b) $\phi=0.7$ with $n=12.5$.

with the receiver on the other side.

Smith \& Kilhams [1963] model of a flame as a distribution of monopolar sound sources therefore appears to be insightful. The simple model in the present work identifies flame annihilation events as these sources and, in so doing, provides a physical explanation for the well known, monopolar content of the sound radiated by turbulent premixed flames.

\subsection{Conclusions}

It was shown qualitatively in the previous chapter that flame annihilation events play a noticeable role in the noise generated by turbulent premixed flames. However, a more detailed analysis was required to quantify the contribution of annihilation events to the sound produced. 
This chapter therefore proposed a simple model that related the observed annihilation events to the far-field sound. This model considered the far-field sound as the superposition of the sound radiated by individual, spherically symmetric annihilation events that occurred throughout the flame. These events were identified by conditioning the flame displacement speed at some threshold since flame annihilation is well known to feature flame acceleration. The choice of threshold was shown to strongly affect the correlation between the modelled and measured, far-field sound, with the correlation coefficient exceeding 0.5 in some cases. Such levels of correlation were surprising given the model's simplicity, and demonstrated the importance of flame annihilation as acoustic sources in turbulent premixed flames. This model is also consistent with Smith \& Kilham's [1963 original model of a flame as a distribution of monopolar sound sources, with the present work identifying flame annihilation events as these sources and, in so doing, providing a physical explanation for the well known, monopolar content of the sound radiated by turbulent premixed flames. 


\section{Chapter 6}

\section{Annihilation Event Statistics in a Turbulent Premixed Flame}

\subsection{Introduction}

There are numerous studies on the effects of strain and curvature on premixed flames (e.g. Matalon \& Matkowsky 1982, Clavin \& Joulin [1983], Searby \& Quinard [1990], Poinsot et al. [1992]), Echekki \& Chen [1996], Najm \& Wyckoff [1997, Chen \& Im 1998, Peters et al. [1998, Echekki \& Chen [1999], Chen et al. [1999], Chen \& Im 2000], Bechtold \& Matalon [2001], Durox et al. [2001], Haq et al. 2002, Sinibaldi et al. [2003], Chakraborty \& Cant [2004], Hawkes \& Chen [2004], Chakraborty \& Cant 2005], Hawkes \& Chen 2005], Hawkes \& Chen [2006], Kerl et al. [2013], Fogla et al. 2015], Sankaran et al. 2015, Ma et al. 2016 ). This subject is of fundamental interest since the flame response to instabilities are modified by these effects. Given the previously-shown significance of flame annihilation events to the sound generated by turbulent premixed flames, it is therefore insightful to study the flame response to strain and curvature during annihilation. The outcomes of this study will have important implications for modelling the flame propagation during annihilation. 


\subsection{Theoretical framework}

As shown in equation 5.1, the flame displacement speed [Echekki \& Chen, 1999] is obtained by the sum of contributions from the reaction rate, the normal diffusion and the flame curvature. These terms are expressed respectively in a non-dimensional form as:

$$
\begin{gathered}
S_{d, r}^{*}=-\frac{\dot{\omega}_{R}}{\rho_{u}\left|\nabla Y_{R}\right|}, \\
S_{d, n}^{*}=-\frac{1}{S c R e} \frac{\mathbf{n} \cdot \nabla\left(\mu\left|\nabla Y_{R}\right|\right)}{\rho_{u}\left|\nabla Y_{R}\right|}, \\
S_{d, c}^{*}=-\frac{\mu}{S c \operatorname{Re} \rho_{u}}(\nabla \cdot \mathbf{n}) .
\end{gathered}
$$

It is again reminded that $(\nabla . \mathbf{n})$ is the flame curvature where $\mathbf{n}$ is the unit normal vector of the iso-contour of $Y_{R}$ directed towards the reactant. The flame curvature is taken to be negative (positive) when the flame is curved towards the fresh reactant (burned mixture).

As mentioned earlier, the response of premixed flames to strain and curvature has been extensively studied. Markstein 1964 proposed the first relationship between flame displacement speed and curvature for small values of curvature,

$$
\frac{S_{d}}{S_{L}}=1-l_{M}(\nabla \cdot \mathbf{n})
$$

where $l_{M}$ is the Markstein length, which depends on the nature of the gaseous mixture, the equivalence ratio, and parameters characterising the fresh and burned gases Durox et al. 2001. After that, theoretical studies of Matalon \& Matkowsky [1982 and Clavin \& Joulin [1983 provided a generalised expression for the flame speed and stretch as follows:

$$
\frac{S_{d}}{S_{L}}=1-\frac{l_{M}}{S_{L}} \kappa
$$

where $\kappa$ is the stretch which represents the relative rate of change of flame surface 
$\operatorname{area}(\mathrm{A})$,

$$
\kappa=\frac{1}{A} \frac{d A}{d t}
$$

The stretch can be expressed exactly as the sum of tangential strain rate, $a_{T}$, and a curvature term (Matalon 1983, Pope 1988, Candel \& Poinsot 1990),

$$
\kappa=a_{T}+S_{d}(\nabla \cdot \mathbf{n})
$$

where $a_{T}=\left(\delta_{i j}-n_{i} n_{j}\right) \partial u_{i} / \partial x_{j}$. It should be noted that equation 6.3 is equivalent to equation 6.2 by neglecting tangential strain rate and for small values of curvature.

The normalised form of equation 6.3 is given by,

$$
\frac{S_{d}}{S_{L}}=1-M a K a
$$

where $M a=l_{M} / \delta_{f}$ is the Markstein number and $\delta_{f}$ is the nominal flame thickness defined in equation 4.1. Clavin \& Joulin 1983 proposed a theoretical relationship for $l_{M} / \delta_{f}$ as follows, which is valid when $L e-1=O(1 / \beta)$ :

$$
\frac{l_{M}}{\delta_{f}}=\frac{1}{\alpha} \ln \frac{1}{1-\alpha}+\frac{\beta(L e-1)}{2}\left(\frac{1-\alpha}{\alpha}\right) \int_{0}^{\alpha /(1-\alpha)} \frac{\ln (1+x)}{x} d x .
$$

Note that $K a$ in equation 6.6 is the normalised stretch known as the Karlovitz number,

$$
K a=K a_{S}+K a_{C}=\frac{\delta_{f}}{S_{L}}\left(a_{T}+S_{d}(\nabla . \mathbf{n})\right),
$$

where $K a_{S}$ and $K a_{C}$ are contributions from tangential strain rate and curvature, respectively. Note that the density-weighted form of equation 6.6 is given by,

$$
\frac{S_{d}^{*}}{S_{L}}=1-M a^{*} K a
$$

with

$$
K a=K a_{S}+K a_{C}=\frac{\delta_{f}}{S_{L}}\left(a_{T}+S_{d}^{*}(\nabla \cdot \mathbf{n})\right),
$$

where $M a^{*}$ is the Markstein number in terms of the density-weighted values of $S_{d}$ 


\subsection{Flame Displacement Speed and Curvature}

In this study a surface-based approach for post-processing of the data is considered. The flame is represented by an iso-surface with $Y_{R}=0.122$ for $\phi=1.0$ and $Y_{R}=0.156$ for $\phi=0.7$. The reason for these choices was discussed in chapter 5. To extract the data on the iso-surface, a triangulation method is used and various statistics are interpolated to the surface points.

Figure 6.1 illustrates the flame surface of three distinct annihilation events: 'island burn-out', 'pinch-off' and 'tunnel formation' events, coloured by different terms described in equation 6.1. As can be seen in figure 6.1, there is a large increase in the magnitude of $S_{d}^{*} / S_{L}$ during annihilation. It is visually understood that the reaction term (figure 6.1 i) clearly plays the most significant role. This is followed by the curvature term (figure 6.1 v) and the normal diffusion term (figure 6.1 iii). However, the relative contribution of these two terms is not qualitatively obvious from this figure.

Figure 6.2 therefore depicts scatter plots of different terms of $S_{d}^{*} / S_{L}$ as a function of normalised curvature $\left(\delta_{t h} \bullet \nabla \cdot \vec{n}\right)$ over the flame surface of the three distinct annihilation events shown in figure 6.1. For all three events, $S_{d}^{*} / S_{L}$ shows a noticeably negative correlation with curvature. The correlation for the island burn-out event (figure 6.2 a) is very strong which might be due to the symmetry present in this type of event. Note that in all three events the reaction and curvature terms increase for higher negative values of curvature, with the normal diffusion term being almost insensitive to the changes in curvature. The contribution of the curvature term is linear as expected from equation 6.1. For high negative values of curvature, the reaction term has clearly the largest contribution to $S_{d}^{*} / S_{L}$, followed by the curvature term and with the least share from the normal diffusion term. However, for small values of curvature there is a competition between all terms for the pinch-off and tunnel formation events only, as shown in figure 6.2 .

In what follows, the results of the last four jet flow through times are considered with 206 and 270 equally spaced time instants for the stoichiometric and lean flames, respectively. Note that the lean case has a bigger jet flow through time because of having a smaller inlet velocity compared to the stoichiometric 
i

a)

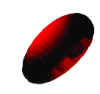

b)

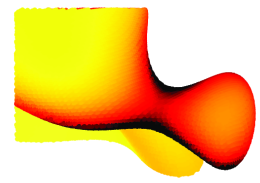

c)

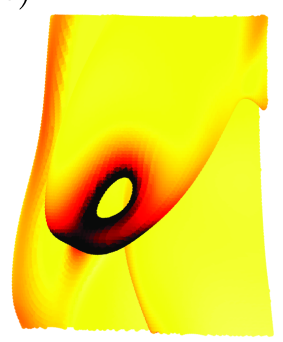

ii

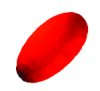

iii

iv
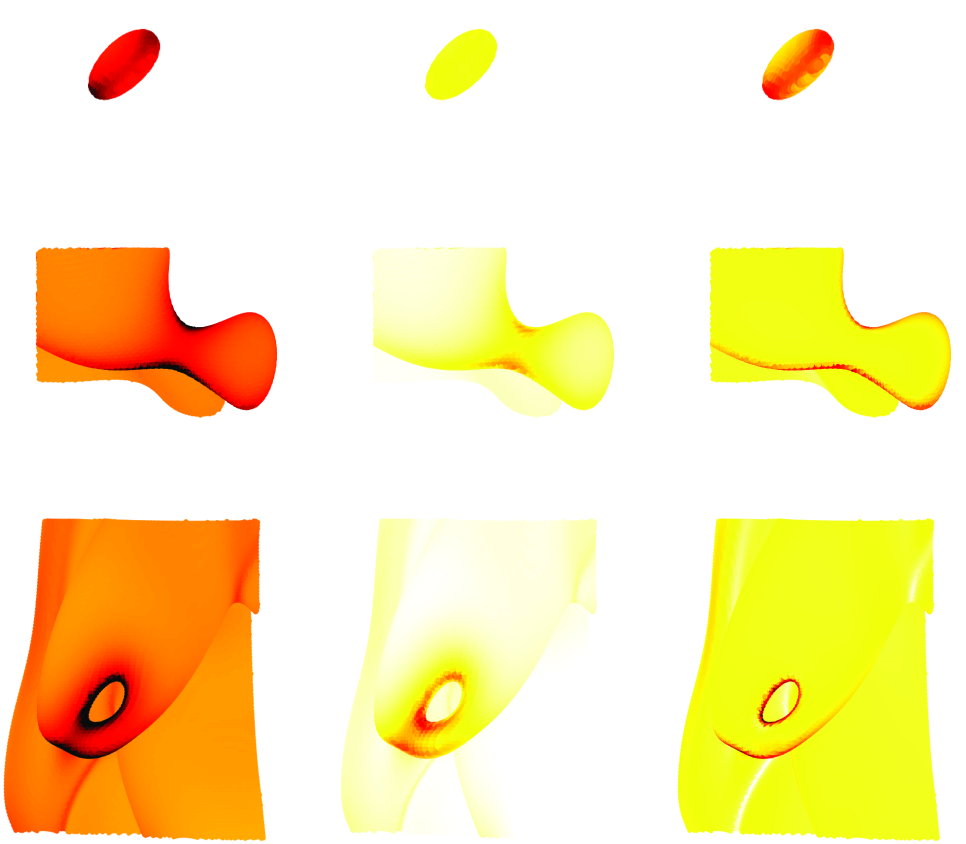

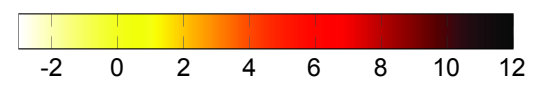

Figure 6.1: The instantaneous distribution of different terms of the normalised density weighted flame displacement speed ( (i) net component, (ii) reaction component, (iii) normal diffusion component and (iv) curvature component) over the flame surfaces of three distinct annihilation events: (a) 'island burn-out', (b) 'pinch-off' and (c) 'tunnel formation' events.

case. It should be noted that the annihilation events are identified using $V_{f}>0$ and the optimal value of $n=S_{d}^{*} / S_{L}$ (equal to 5 for $\phi=1.0$ and 12.5 for $\phi=0.7$ ) found in Chapter 5.

First, the probability density function (PDF) of the flame surface curvature normalised with the laminar flame thickness $\left(\delta_{t h} \bullet \nabla . \vec{n}\right)$ for the entire flame surface is shown in figure 6.3. As can be seen in figure 6.3 the same statistical distributions with approximately symmetric PDFs around zero, being slightly skewed towards negative values with a mean value at -0.2 Sankaran et al. 2015 performed DNS of turbulent premixed slot-jet flames and showed that the curvature distribution was not symmetric and the probability of 
(a)

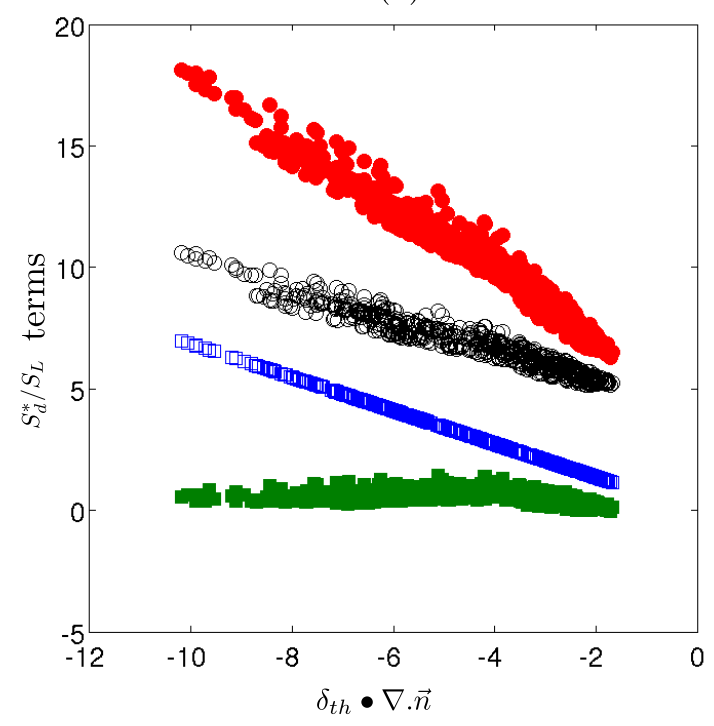

(b)

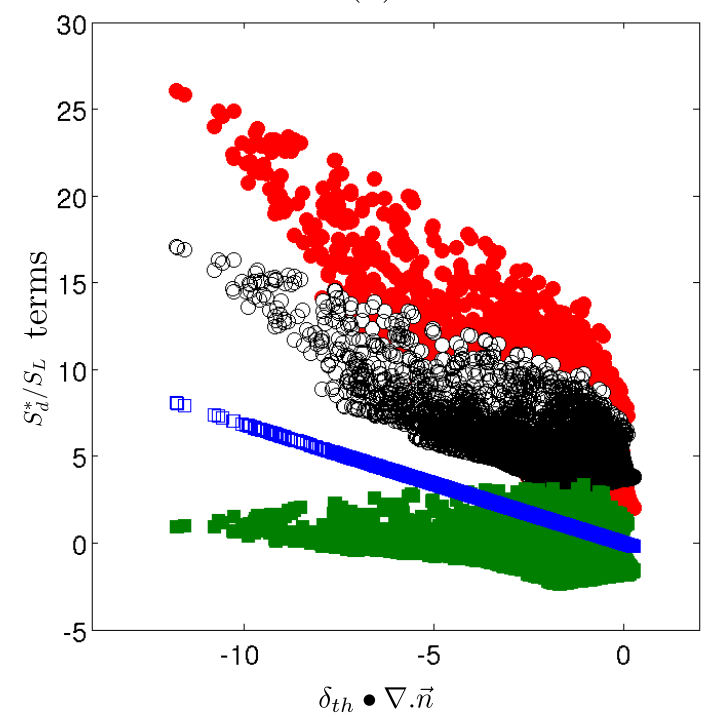

(c)

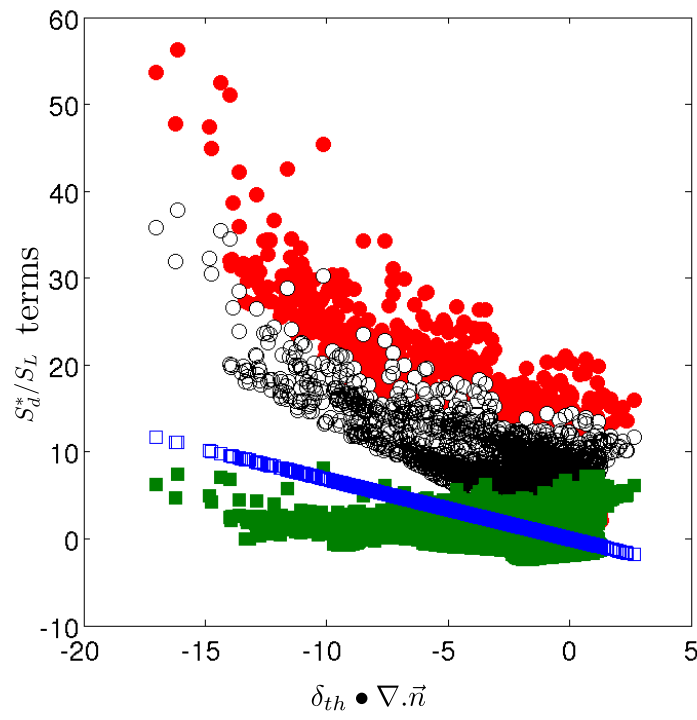

Figure 6.2: Scatter plots of different terms of the normalised density-weighted flame displacement speed ( (solid circle) net component, (hollow circle) reaction component, (solid square) normal diffusion component and (hollow square) curvature component) versus the normalised curvature $\left(\delta_{t h} \bullet \nabla . \vec{n}\right)$ over the flame surfaces of the three distinct annihilation events shown in figure 6.1 (a) "island burn-out', (b) 'pinch-off' and (c) 'tunnel formation' events.

high positive curvatures was higher than high negative curvatures for their flames studied. However, it appears that their conclusions cannot be extended to the 
(a)

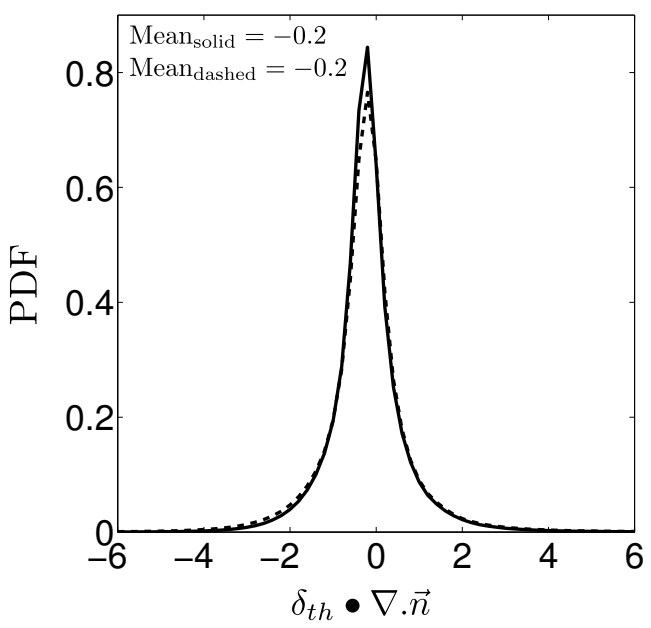

(b)

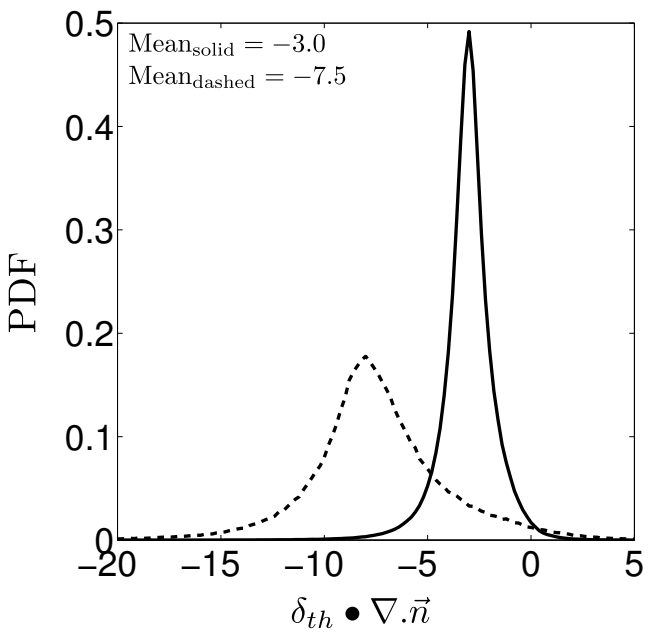

Figure 6.3: PDFs of the normalised curvature $\delta_{t h} \bullet \nabla . \vec{n}$ for (a) entire flame and (b) considering only annihilation events: $\phi=1.0$ (solid) and $\phi=0.7$ (dashed).

turbulent premixed round-jet flames investigated in the present study.

With regard to the curvature distribution for the annihilation events, it is observed in figure 6.3b that both flames feature negative values of curvature (centre of curvature is in the unburned mixture), with the lean flame covering higher negative values. The latter is because the lean flame was conditioned on a higher $S_{d}^{*} / S_{L}$ value for identifying the annihilation events and highly curved flame surfaces are expected to be captured. This explains that the flame-generated noise for $\phi=0.7$ is mainly due to highly curved flames towards reactants.

Figure 6.4 shows the PDFs of different terms contributing to $S_{d}^{*} / S_{L}$ for the whole flame surface for both cases. It is observed in figure 6.4 that the PDF of $S_{d}^{*} / S_{L}$ lies significantly on the positive side with a mean value at 1.0 for both flames. However, the negative values may be achieved due to strong turbulent eddies (excessive tangential straining) Chen \& Im, 1998; Libby, 1994 through pushing the flame front to move towards the burned mixture. Figure 6.4a also suggests that annihilation events constitute a significantly smaller statistics compared with the whole flame surface for both flames. Nonetheless, flame annihilation events play a significant role in the noise generated by turbulent premixed flames, as shown in previous chapters.

The PDFs of the contributions due to reaction rate $\left(S_{d, r}^{*} / S_{L}\right)$, normal diffusion 
$\left(S_{d, n}^{*} / S_{L}\right)$ and curvature $\left(S_{d, c}^{*} / S_{L}\right)$ for the whole flame surface are also depicted in figure 6.4 $\mathrm{b}$-d. It can be seen that the PDF of $S_{d, r}^{*} / S_{L}$ is always positive with a mean value at 3.3 for the stoichiometric flame and at 2.6 for the lean flame. On the contrary, $S_{d, n}^{*} / S_{L}$ exhibits large negative values for both flames, with a mean value at -2.4 for the stoichiometric flame and at -1.7 for the lean flame. The small difference between the mean values for the two flames is due to different iso-surfaces of the progress variable $Y_{R}$ representing the flames, with that of the stoichiometric flame being slightly closer to the burned mixture $\left(Y_{R}=0.122\right.$ for $\phi=1.0$ compared with $Y_{R}=0.156$ for $\left.\phi=0.7\right)$. This is consistent with Chakraborty \& Cant 2004] showing that the probability of higher negative values of $S_{d, n}^{*} / S_{L}$ and higher positive values of $S_{d, r}^{*} / S_{L}$ is achieved as the progress variable approaches the burned gas side. As shown in figure $6.4 \mathrm{~d}, S_{d, c}^{*} / S_{L}$ is nearly symmetric around zero, being slightly skewed towards positive values, which is consistent with the PDF of curvature shown in figure 6.3 a. The PDFs for annihilation events featured a similar shape to those shown in 6.4 (not shown here) with noticeable shifts towards $S_{d}^{*} / S_{L}=n$ due to conditioning of the data on threshold $n$.

Figure 6.5 shows the conditional mean of the percentage contribution of different terms for $S_{d}^{*} / S_{L}$ as a function of the normalised curvature for the whole flame surface and for the annihilation events. In order to analyse the results presented in figure 6.5, different ranges of curvature will be considered. First for the range of $-3<\delta_{t h} \bullet \nabla \cdot \vec{n}<3$ (which contains most of the statistics for the whole flame surface, as shown in figure 6.3), it is understood that the reaction term has the highest contribution among the other components at around $60 \%$. This is followed by the normal diffusion term which peaks just above $40 \%$ at zero curvature. Unsurprisingly, the curvature component exhibits a stronger contribution at higher values. For annihilation events for which the range of interest for curvature is $-15<\delta_{t h} \bullet \nabla \cdot \vec{n}<0$ (see figure 6.3), the reaction component still plays a major role, attaining about $60 \%$ for highly negative values of curvature. Moving towards the zero curvature, this contribution increases to about $90 \%$ for $\phi=1.0$ and $70 \%$ for $\phi=0.7$ at zero curvature. This behaviour for small curvatures can be attributed to the tunnel formation events which are approximately planar like. It is interesting to note that on average the diffusion term has the 
(a)

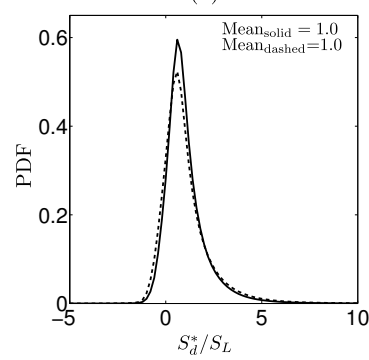

(b)

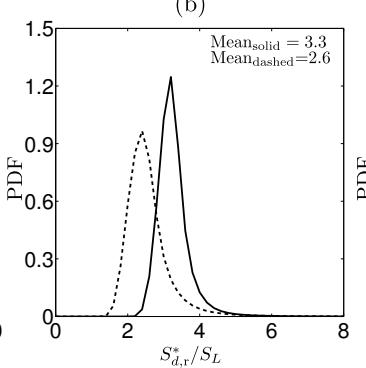

(c)

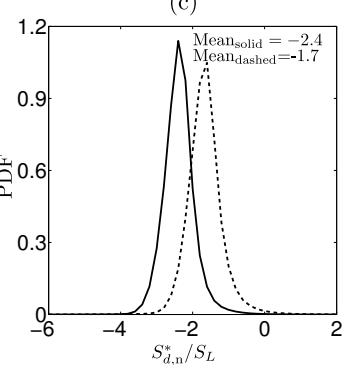

(d)

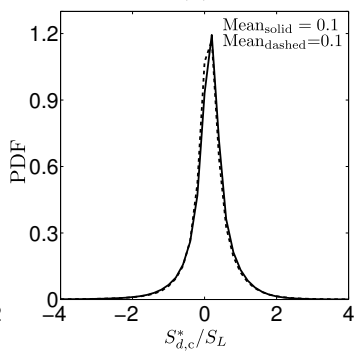

Figure 6.4: PDFs of flame displacement speed components for $\phi=1.0$ (solid) and $\phi=0.7$ (dashed) over entire flame: (a) net component, (b) reaction component, (c) normal diffusion component and (d) curvature component.

(a)

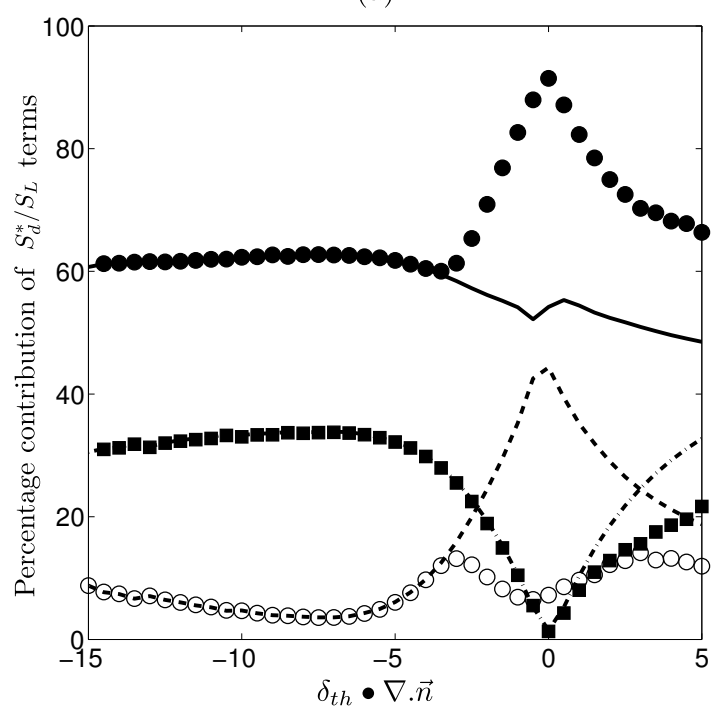

(b)

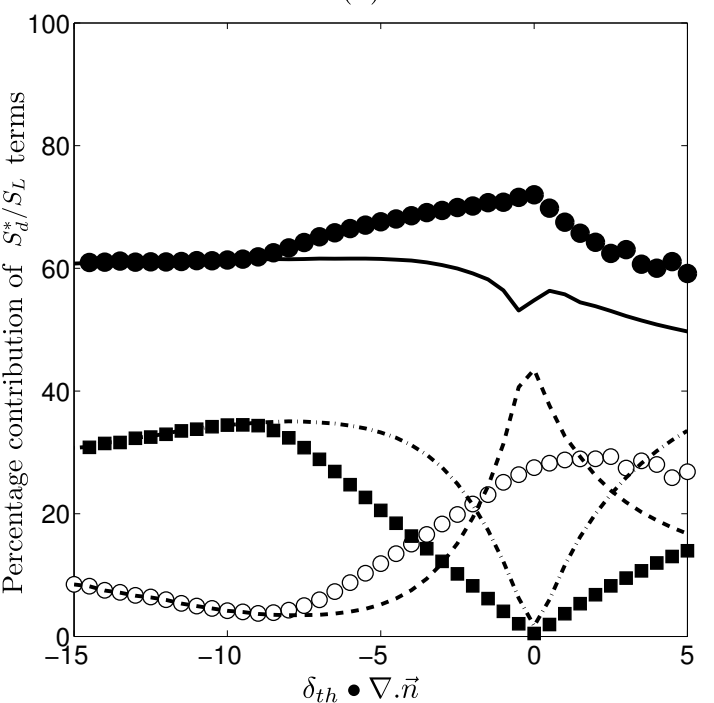

Figure 6.5: Conditional mean of percentage contribution of the absolute values of different terms of $S_{d}^{*} / S_{L}$ versus the normalised curvature for entire flame ((solid line) reaction component, (dashed) normal diffusion component and (dash-dot) curvature component)) and for annihilations events ((solid circle) reaction component, (hollow circle) normal diffusion component and (solid square) curvature component): (a) $\phi=1.0$ and (b) $\phi=0.7$.

least contribution compared with other terms for annihilation events when $\phi=1.0$. However, for the lean flame, the diffusion term is relatively stronger for small values of curvature. Overall, this figure highlights the significance of the reaction rate term for annihilation events, in particular for small values of curvature.

Figure 6.6 shows the joint PDFs (JPDFs) between different $S_{d}^{*} / S_{L}$ terms and 
a)
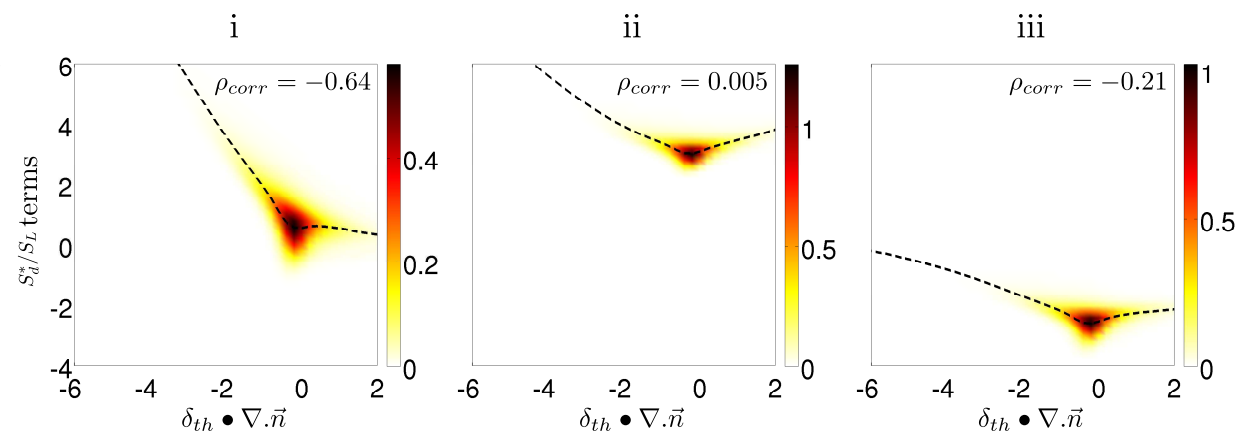

b)
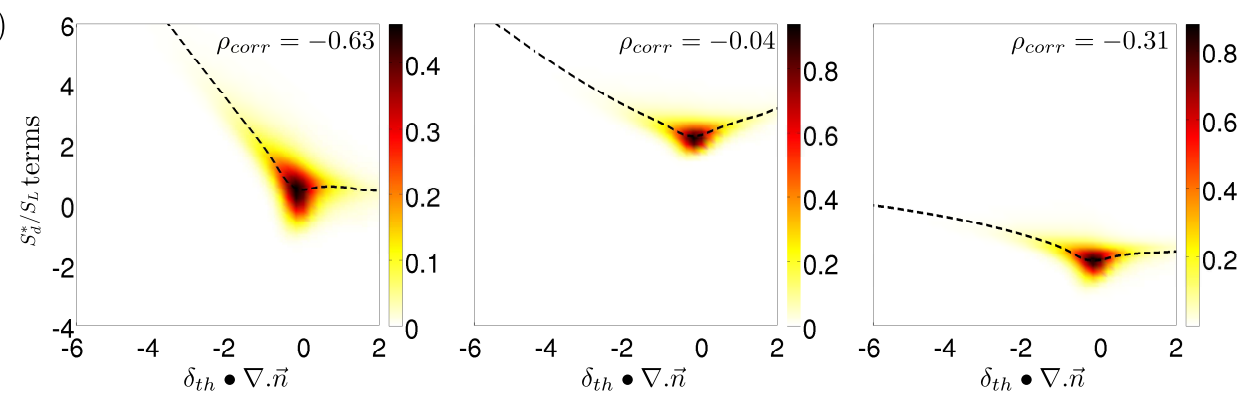

c)
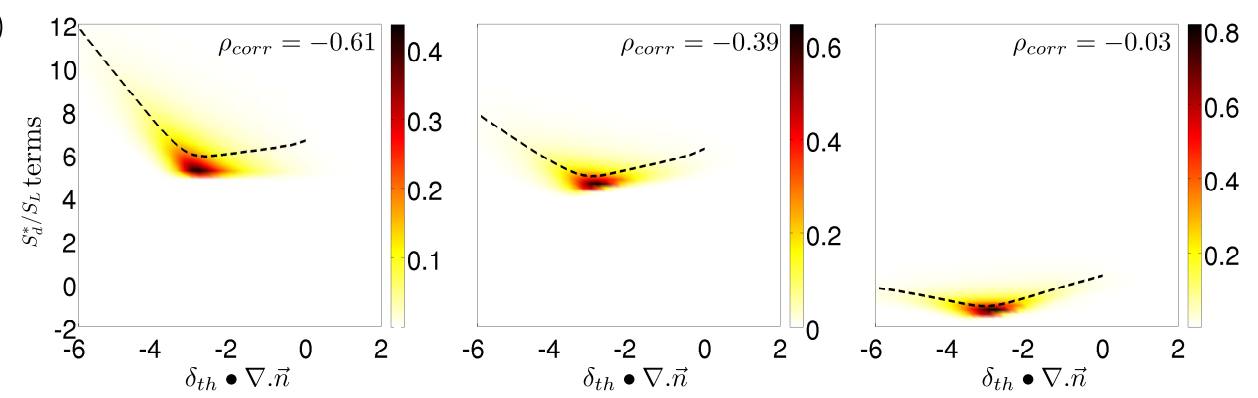

d)

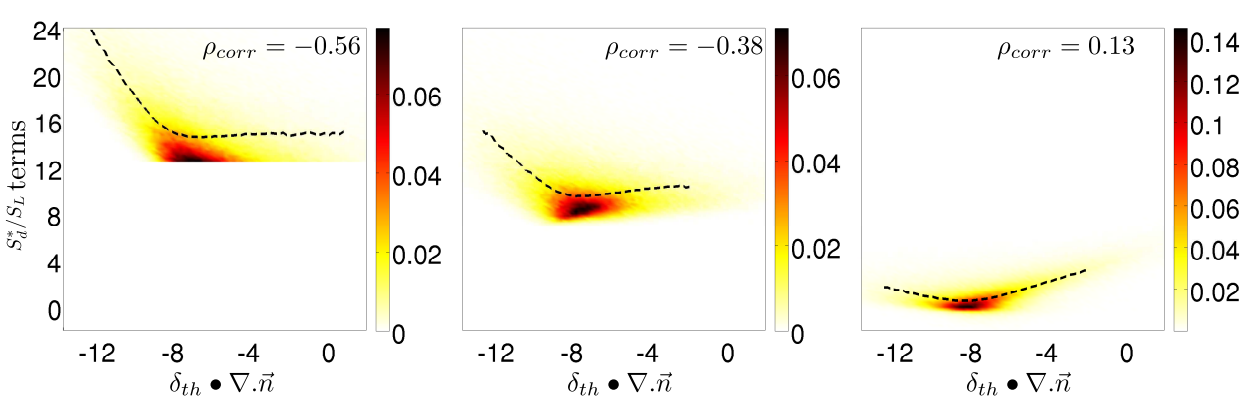

Figure 6.6: Correlation of different terms of the normalised density-weighted flame displacement speed $\left(S_{d}^{*} / S_{L}\right)$ with normalised curvature $\left(\delta_{t h} \bullet \nabla \cdot \vec{n}\right)$ for entire flame $(\phi=1.0$ (a) and $\phi=0.7$ (b)) and considering only annihilation events $(\phi=1.0$ (c) and $\phi=0.7$ (d)): i) net term, ii) reaction term and iii) normal diffusion term. Dashed lines show curvature conditional means of $S_{d}^{*} / S_{L}$ terms 
$\delta_{t h} \bullet \nabla . \vec{n}$ for both the whole flame surface and annihilation events. The curvature conditional mean of $S_{d}^{*} / S_{L}$ terms, shown as a dashed line, is also presented. Note that the linear correlation between $S_{d, c}^{*} / S_{L}$ and curvature is obvious and is not shown here. Considering the entire flame data as observed in figure 6.6 $\mathrm{a}$ and $\mathrm{b}$, both flames exhibit almost the same correlation between $S_{d}^{*} / S_{L}$ and $\delta_{t h} \bullet \nabla \cdot \vec{n}$, with a correlation coefficient $\rho \approx-0.6$. As can be observed, strongly negative values of the curvature result in very high values of the displacement speed. These results are qualitatively consistent with previous findings for two-dimensional DNS with detailed chemistry Chen \& Im, 1998; Echekki \& Chen, 1996; Peters et al., 1998], three-dimensional DNS of inflow/outflow configuration with single step chemistry Chakraborty \& Cant, 2004, and DNS of turbulent premixed slot-jet flames Sankaran et al. 2015. It is also seen that there is almost no correlation between the reaction term and the curvature, while a weak negative correlation is observed for the normal diffusion term and the curvature. This observation suggests that the negative correlation between $S_{d}^{*} / S_{L}$ and curvature is mostly due to the curvature term. It should be noted that the reaction term (see figure 6.6 i of the entire flame data) shows different behaviours depending on the magnitude and sign of the curvature. That is, for positive and slightly negative values of curvature, $S_{d, r}^{*} / S_{L}$ and $\delta_{t h} \bullet \nabla \cdot \vec{n}$ are positively correlated, whereas a negative correlation is observed for high negative values of curvature. This is consistent with the findings of Echekki \& Chen [1999] in their DNS study.

Now considering only the annihilation events, figure 6.6 and d show the effect of curvature on different $S_{d}^{*} / S_{L}$ terms. In comparison with the statistics for the whole flame surface, there is no significant change in the correlation between $S_{d}^{*} / S_{L}$ and $\delta_{t h} \bullet \nabla . \vec{n}$. However, the correlation coefficient increases by about $40 \%$ for the reaction term, while the normal diffusion term becomes almost uncorrelated with the curvature. A comparison of figures 6.5 and 6.6 highlights the negligible role of the diffusion term for annihilation events, in particular for $\phi=0.7$.

The reason behind these observations is now investigated. Equation 6.1 shows that the reaction term, $S_{d, r}^{*} / S_{L}$, is proportional to the reciprocal of the magnitude of the reactant mass-fraction gradient $\left(1 /\left|\nabla Y_{R}\right|\right)$ multiplied by reaction rate $\left(\dot{\omega}_{R}\right)$. Since a one-step chemistry model with unity Lewis number is used and the flame 
is also represented at a fixed reactant mass-fraction iso-surface, the reaction rate itself experiences small fluctuations due to compressibility effects only. Figure 6.7 confirms this behaviour as the conditional mean of $\dot{\omega}_{R}$ on curvature is almost invariant, and $\dot{\omega}_{R}$ is uncorrelated with curvature for both the whole flame surface and the annihilation events. Note that only the results of $\phi=1.0$ are depicted due to similar behaviours observed for two flames as previously shown in figure 6.6. Further, as shown in figure 6.7 i, $\left|\nabla Y_{R}\right|$ is uncorrelated with curvature for the whole flame surface, whereas a level of correlation at $31 \%$ is seen for annihilation events. It is therefore understood that the noticeable increase in the correlation between $S_{d, r}^{*} / S_{L}$ and $\delta_{t h} \bullet \nabla \cdot \vec{n}$ for annihilation events (see figure 6.6ii) is attributed to $1 /\left|\nabla Y_{R}\right|$. This observation may be attributed to the depletion of reactants at very high values of curvature $\left(1 /\left|\nabla Y_{R}\right| \longrightarrow \infty\right)$.

With regard to the normal diffusion term, $S_{d, n}^{*} / S_{L}$, equation 6.1 shows its relation with $1 /\left|\nabla Y_{R}\right|$ as well as the second derivative of the mass fraction in the normal direction $\left(\mathbf{n} . \nabla\left(\mu\left|\nabla Y_{R}\right|\right)\right)$. Considering the results shown in figure 6.7, it becomes clear that the latter plays an important role in correlation of $S_{d, n}^{*} / S_{L}$ with curvature for the entire flame. However, given the drop of about $20 \%$ in the correlation for the normal diffusion term with curvature (see figure 6.6 iii) for the flame annihilation, it is inferred that the second derivative term counteracts the effect of $1 /\left|\nabla Y_{R}\right|$ during annihilation events.

Figure 6.8 depicts how the correlation between the $S_{d}^{*} / S_{L}$ terms and curvature varies along the jet axial direction for annihilation events. Note that the linear correlation between $S_{d, c}^{*} / S_{L}$ and curvature is obvious and is not shown here. As can be seen for $x / D>12$ there is a significant increase in the correlation coefficient, in particular for $S_{d}^{*} / S_{L}$ and $S_{d, r}^{*} / S_{L}$ reaching a value close to 100 percent. 'Island burn-out' events have arguably a larger contribution to the statistics in this range $(x / D>12)$ compared with flame annihilation events such as 'tunnel formation' and 'pinch-off' events. This very strong correlation is therefore expected due to the resemblance of island burn-out events to a spherically symmetric annihilation event. 
(a)
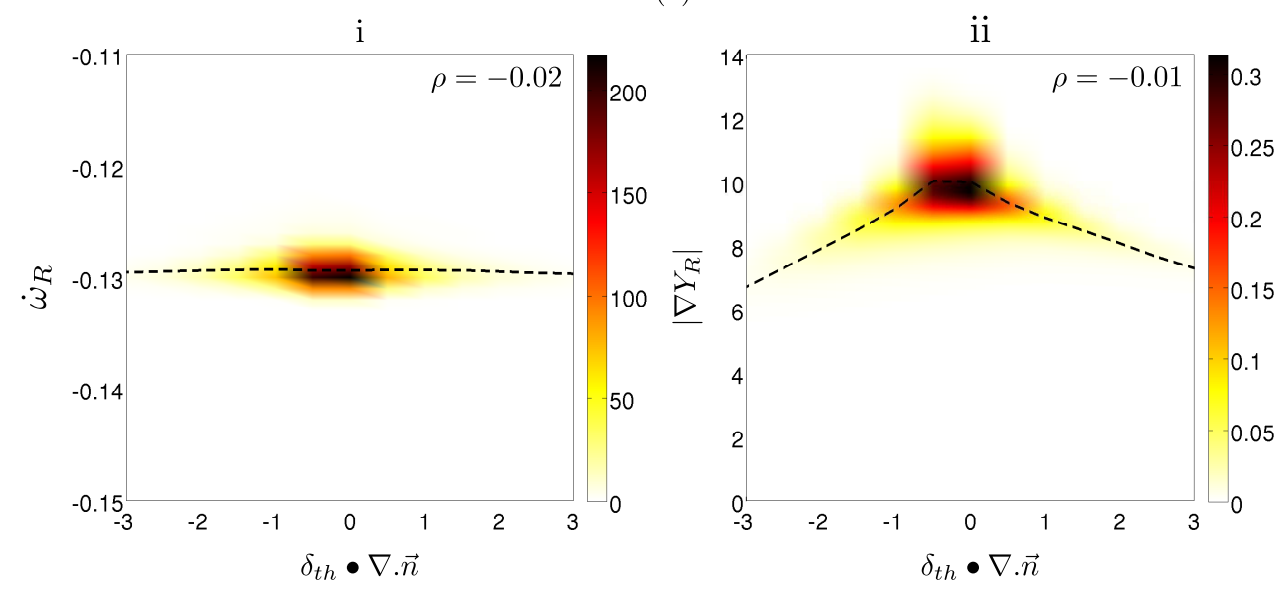

(b)
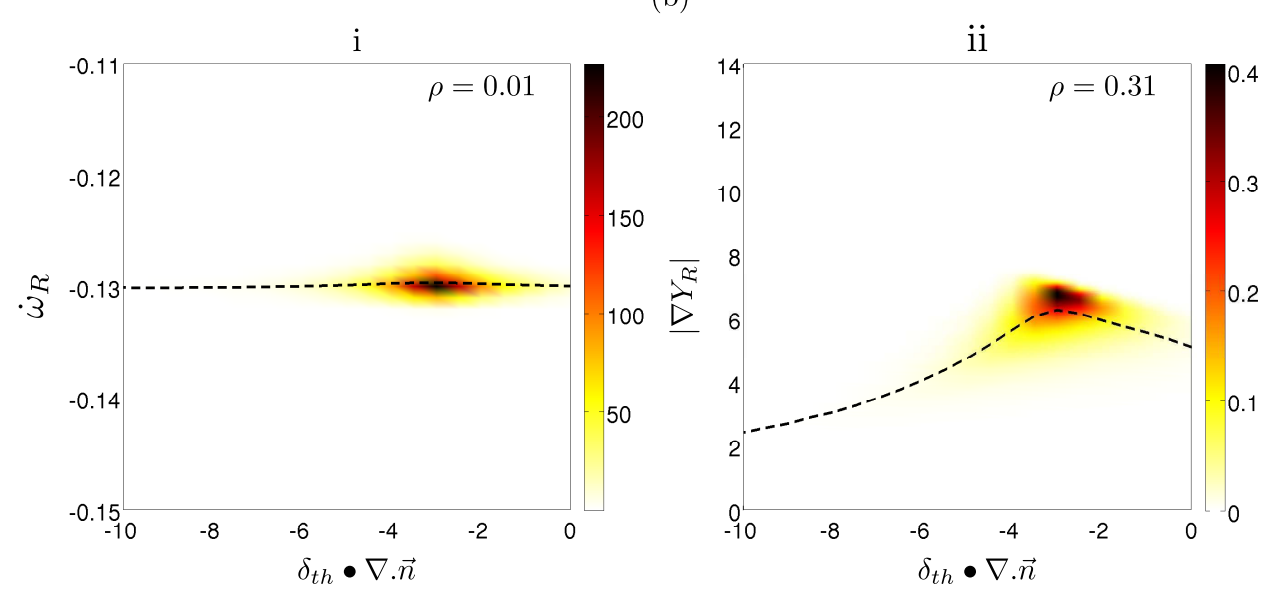

Figure 6.7: The joint PDFs with normalised curvature $\left(\delta_{t h} \bullet \nabla . \vec{n}\right)$ of (i) reaction rate $\dot{\omega}_{R}$ and (ii) the magnitude of reactant mass-fraction gradient $\left|\nabla Y_{R}\right|$ for the case with $\phi=1.0$ : (a) entire flame and (b) annihilation events.

\subsection{Flame Stretch and Markstein Number}

So far the response of turbulent premixed flames (flame displacement speed) to curvature was statistically examined. However, the knowledge of stretch, rate of change of flame surface area, and its relationship with flame displacement speed during flame annihilation are also of importance in terms of modelling turbulent combustion and flame-generated noise. Indeed, it is not clear that if the asymptotic theory (equation 6.6) is valid for flame annihilation events, in view of the fact that this linear theory was derived based on the assumption of small 
(a)

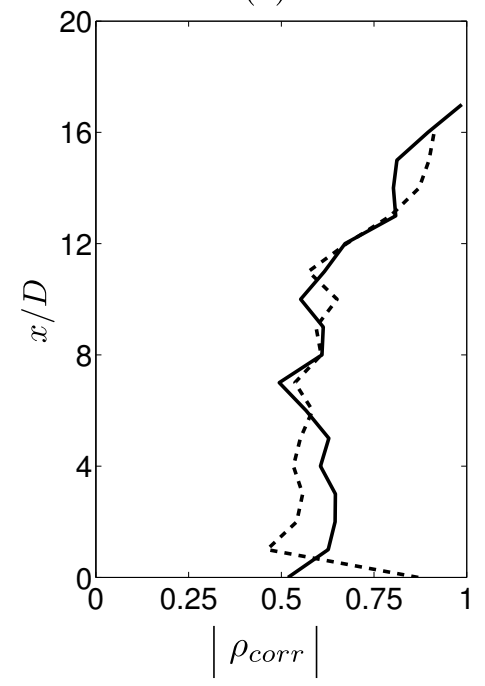

(b)

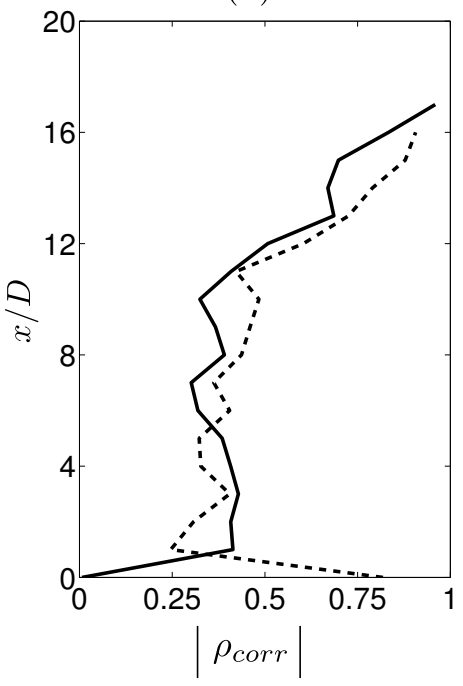

(c)

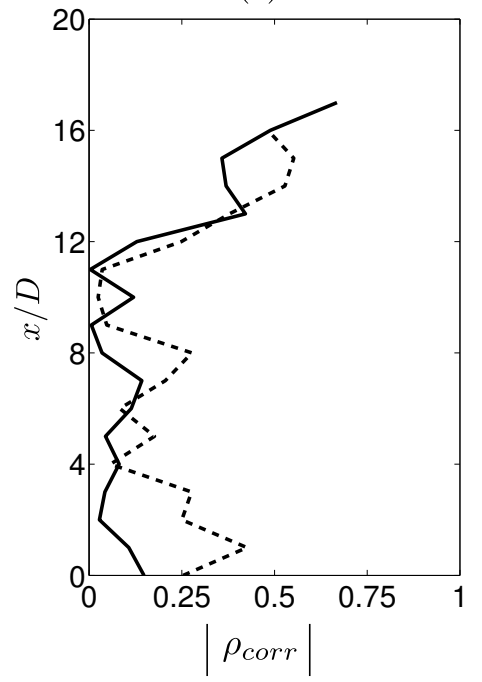

Figure 6.8: Correlation coefficient between different terms of the normalised density weighted flame displacement speed ((a) net term, (b) reaction rate term and (c) normal diffusion term) and the normalised curvature conditioned on the streamwise direction $x / D$ and considering only annihilation events for $\phi=1.0$ (solid) and $\phi=0.7$ (dashed).

(a)

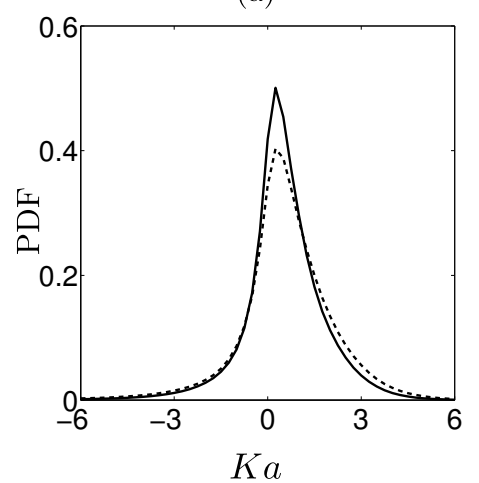

(b)

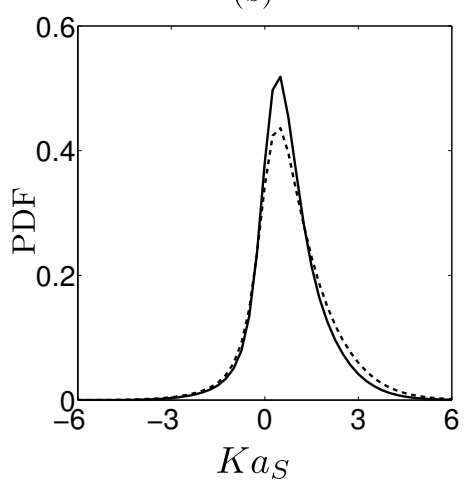

(c)

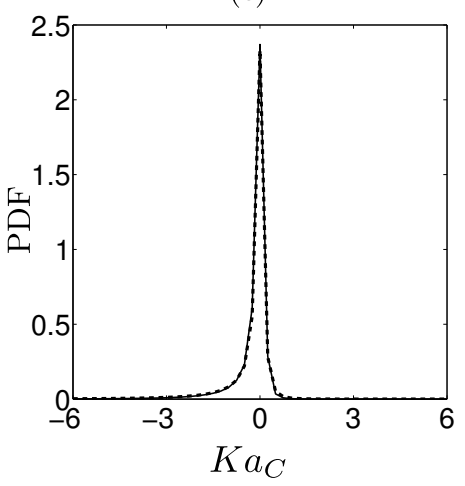

Figure 6.9: PDFs of the normalised flame stretch for $\phi=1.0$ (solid) and $\phi=0.7$ (dashed) with no condition on $S_{d}^{*} / S_{L}$ : (a) $K a$, (b) $K a_{S}$ and (c) $K a_{C}$.

values of stretch. This is the purpose of the research conducted in this section.

Figure 6.9 shows the PDFs of the normalised flame stretch $(\mathrm{Ka})$ and the contributions from tangential strain rate $\left(K a_{S}\right)$ and curvature $\left(K a_{C}\right)$ for the whole flame surface. It is seen from figure 6.9a that the PDF of $K a$ is visibly skewed towards positive values with a mean value close to 0.5 for both flames. There is 
(a)

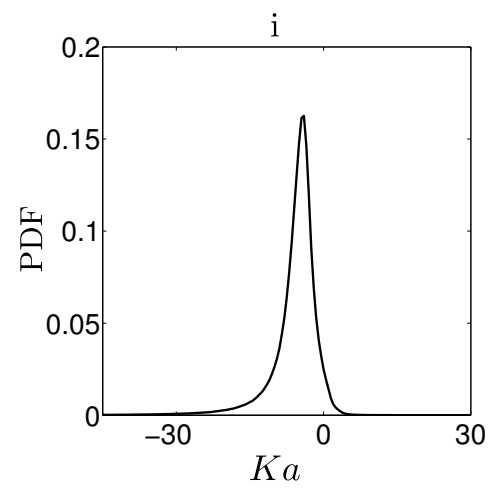

ii
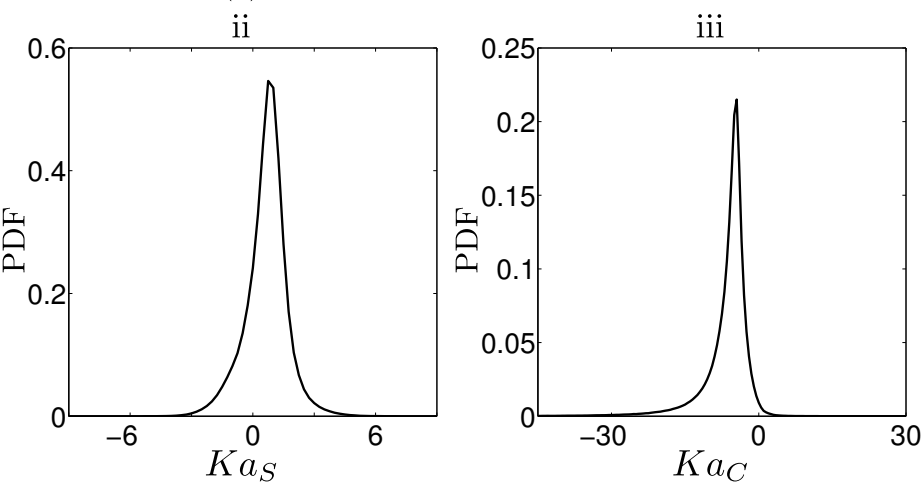

(b)
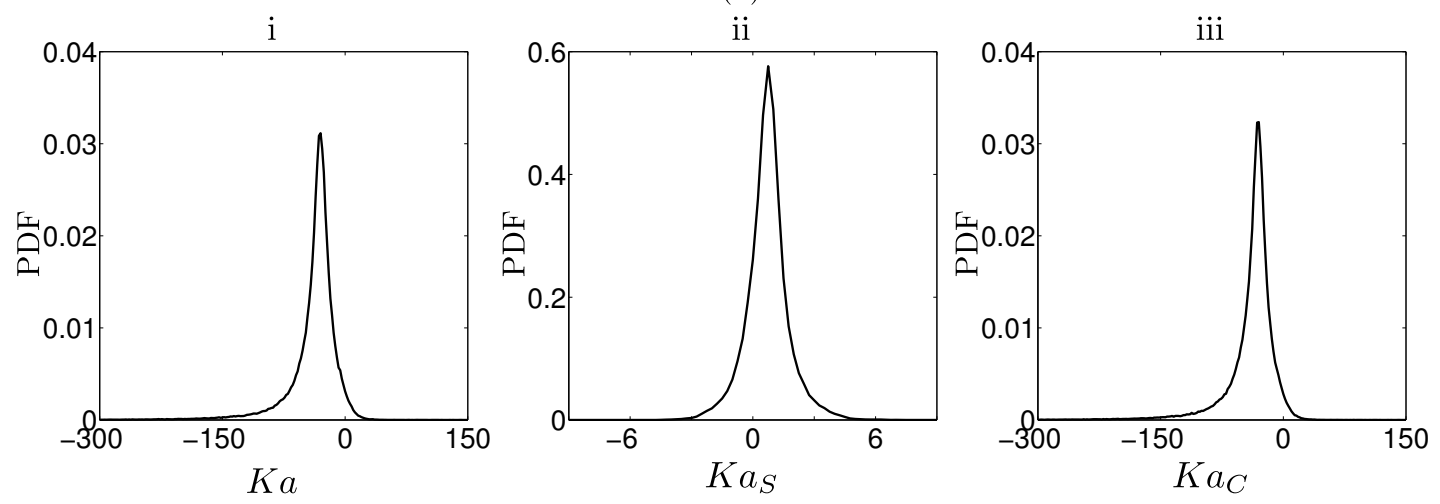

Figure 6.10: PDFs of the normalised flame stretch for (a) $\phi=1.0$ and (b) $\phi=0.7$ considering only the annihilation events: (i) $K a$, (ii) $K a_{S}$ and (iii) $K a_{C}$.

a significant contribution from the tangential strain rate term (figure 6.9p) compared to the curvature term (figure 6.9k). Note that the latter component $\left(K a_{C}\right)$ has a tendency towards the negative values with a mean value around -0.3 for both flames. Overall, this figure indicates that the tangential strain rate is mainly the cause of flame surface generation (positive stretch), whereas the curvature component results in flame surface dissipation (negative stretch). However, the flame surface creation unsurprisingly prevails here where the whole flame statistics is analysed. It is worth mentioning that the fraction of flame surface area undergoing compression, or negative stretch, is $30 \%$ for both flames. Kostiuk \& Bray 1993 performed statistical analysis of propagating surfaces from constant density DNS dataset and showed that a large portion (30-50\%) of flames studied was under compression. Interestingly, Chen \& Im [1998] also reported the magnitude of $30 \%$ from DNS of two-dimensional premixed flames with detailed chemistry. 
Now considering only the annihilation events, figure 6.10 shows that the PDF of $K a$ lies on the negative side (as opposed to the entire flame) with a mean value of -6 for the stoichiometric flame and -40 for the lean flame. These highly negative values are due to significant destruction of the flame surface area during flame annihilation events. It should be noted that this huge difference between the means in two flames is due to conditioning the data on different values of $n=S_{d}^{*} / S_{L}$, as the optimal threshold. In contrast to the whole flame statistics, it should be also stated that the curvature component plays a significant role in $K a$, with a notably minimal contribution form the tangential strain rate being skewed towards positive values.

Figure 6.11 depicts the correlation of flame displacement speed, $S_{d}^{*} / S_{L}$, with the Karlovitz number, $K a$, for both the whole flame surface and the annihilation events. The conditional mean of $S_{d}^{*} / S_{L}$ on $K a$, shown as a dashed line, is also presented. A strong negative correlation between $S_{d}^{*} / S_{L}$ and $K a$ is established for both flames, with a correlation coefficient $\rho \approx-0.7$. As can be observed, highly positive values of $K a$ coincides with small values of $S_{d}^{*} / S_{L}$, implying that the flame area growth (i.e. positive stretch) is a slow process. However, flame surface dissipation (i.e. negative values of stretch) coincides with large values of the displacement speed. Now considering only the annihilation events, figure 6.11ii shows that there are increases of about $10 \%$ and $20 \%$ in the correlation between $S_{d}^{*} / S_{L}$ and $K a$ for the stoichiometric flame and the lean flame, respectively. This suggests that assuming a nearly linear relationship between the response of turbulent premixed flames to stretch is more applicable when only the annihilation events are considered.

The Markstein number $M a^{*}$ used in equation 6.9 can be obtained by a leastsquares linear fit over the DNS data. The calculated $M a^{*}$ is equal to 0.57 and 0.54 for $\phi=1.0$ and 0.7 , respectively. In DNS of two-dimensional unsteady premixed methane/air flames with detailed chemistry for $\phi=0.7$ and 1 and various levels of $u^{\prime} / S_{L}$, Chen \& Im 1998 reported $M a^{*}$ ranging from 0.74 to 0.92. Table 6.1a summarises the above and also shows $M a$ calculated from the theory (equation 6.7 Clavin \& Joulin, 1983]) and from DNS of a spherically symmetric flame annihilation where $r_{0} / \delta_{t h}>2$. There is a good agreement between the theory and the results for the simple flame geometry. However, the theory of Clavin \& 
(a)
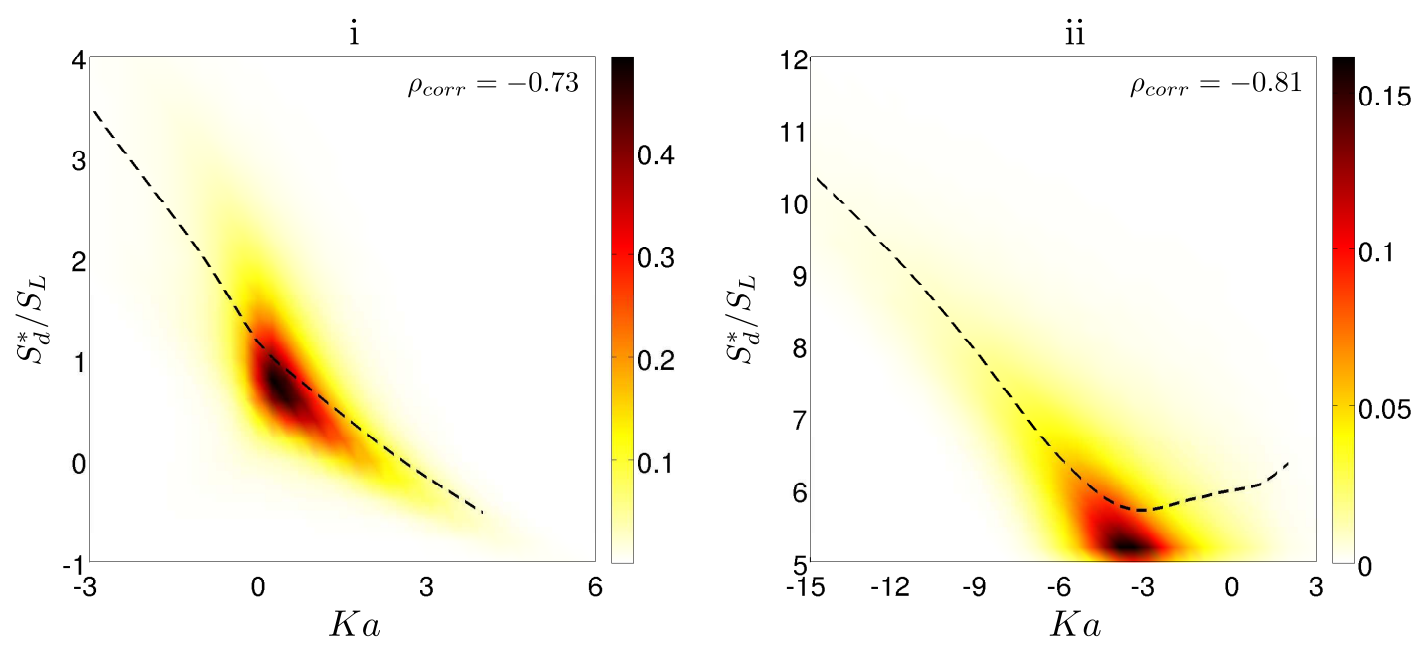

(b)
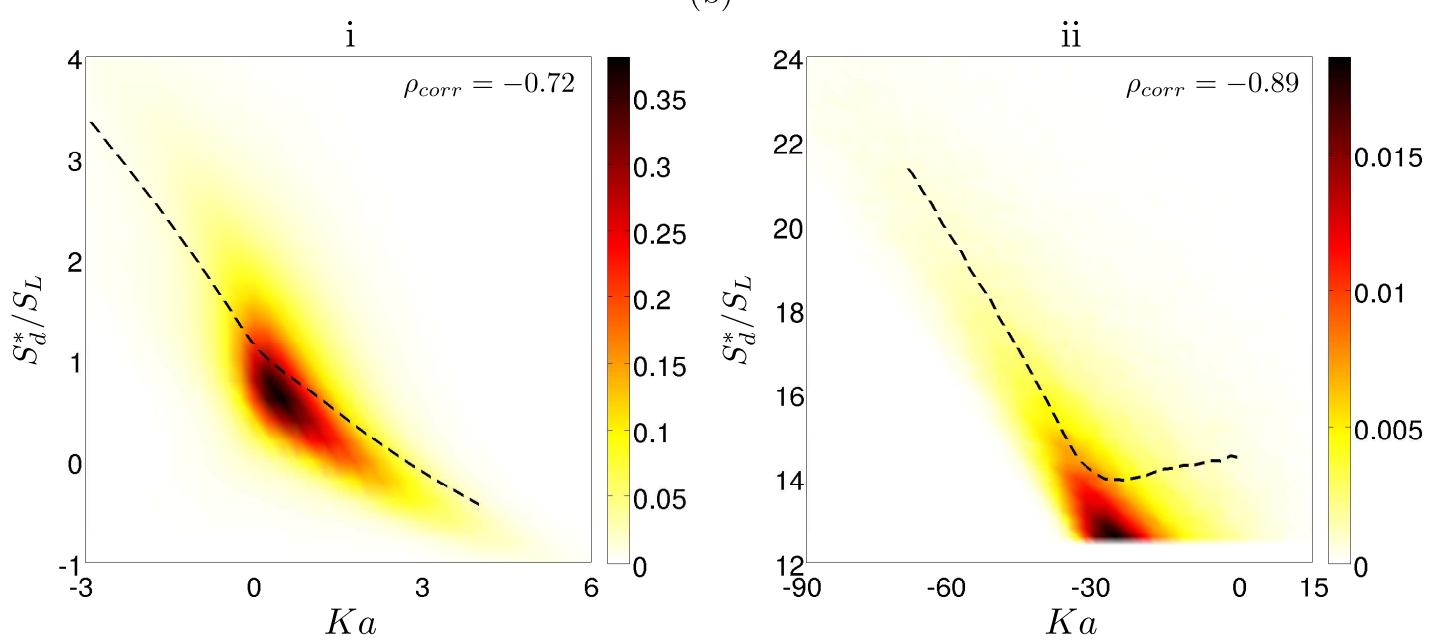

Figure 6.11: Correlation of the normalised flame stretch $(\mathrm{Ka})$ with $S_{d}^{*} / S_{L}$ for (a) $\phi=1.0$ and (b) $\phi=0.7$ : (i) whole flame surface statistics and (ii) considering only the annihilation events. The dashed lines show the $K a$ conditional mean of $S_{d}^{*} / S_{L}$.

Joulin 1983 overpredicts the $M a$ calculated for the turbulent premixed flames, with a maximum overprediction of about $40 \%$.

With regard to the flame annihilation events, $M a^{*}$ equal to 0.31 and 0.13 are obtained for $\phi=1.0$ and 0.7 , respectively, as shown in table 6.1b. Note that the calculated $M a$ is very close to $M a^{*}$. This is expected since in the limit of $S_{d}^{*} / S_{L}>>1$ and the tangential strain rate being negligible $\left(S_{d}^{*}(\nabla \cdot \mathbf{n})>>a_{T}\right)$, it 
Table 6.1: Calculated Markstein numbers from (a) turbulent premixed flames under no condition, spherically symmetric flame $\left(r_{0} / \delta_{t h}>2\right)$ and theory (Eq 6.7) and (b) considering only turbulent premixed flame annihilation events.

(a)

\begin{tabular}{ccccc}
\hline \hline$\phi$ & $M a^{*}$ & $M a$ & $M a$ (Sph. Symm.) & $M a$ (Eq. 6.7) \\
\hline 1.0 & 0.57 & 1.28 & 1.52 & 1.66 \\
0.7 & 0.54 & 1.13 & 1.37 & 1.59 \\
\hline
\end{tabular}

(b)

\begin{tabular}{ccc}
\hline \hline$\phi$ & $M a^{*}$ & $M a$ \\
\hline 1.0 & 0.31 & 0.35 \\
0.7 & 0.13 & 0.13 \\
\hline
\end{tabular}

can be readily shown that $M a^{*}$ is approximately equal to $M a$.

In order to improve the predictions of the linear theory for annihilation events, equation 6.6 can be modified as follows:

$$
S_{d}^{*} / S_{L}=n-M a^{*} K a .
$$

This modification is expected to improve the model prediction since a strong correlation (over $80 \%$ ) between $K a$ and $S_{d}^{*} / S_{L}$ for annihilation events (identified by the selected threshold $n$ ) was observed, as shown in figure 6.11. This suggests that the asymptotic theory may also be valid over a broader range of $K a$ achieved during flame annihilation, which was based on the assumption of small values of stretch $(K a<<O(1))$. Figure 6.12 confirms this hypothesis showing that the new proposed linear model (equation 6.11) is a better representation of annihilation events for both flames. 
(a)

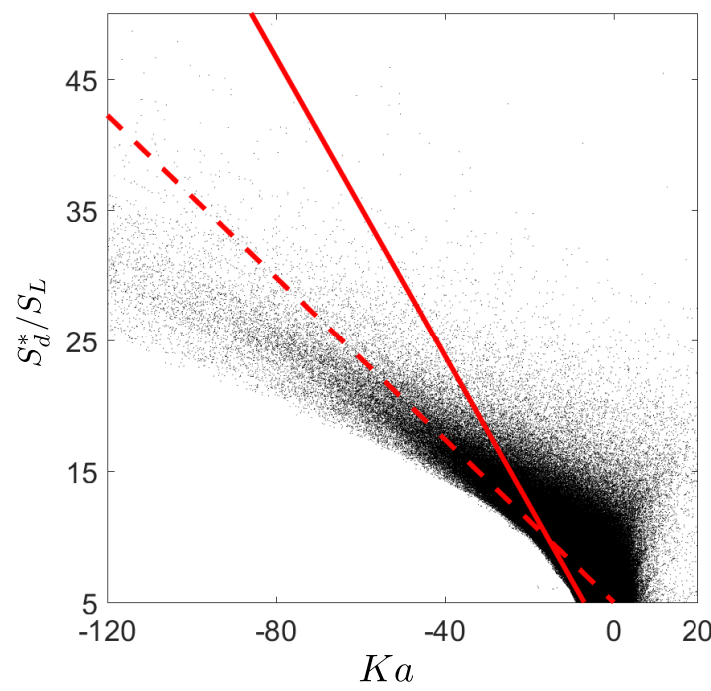

(b)

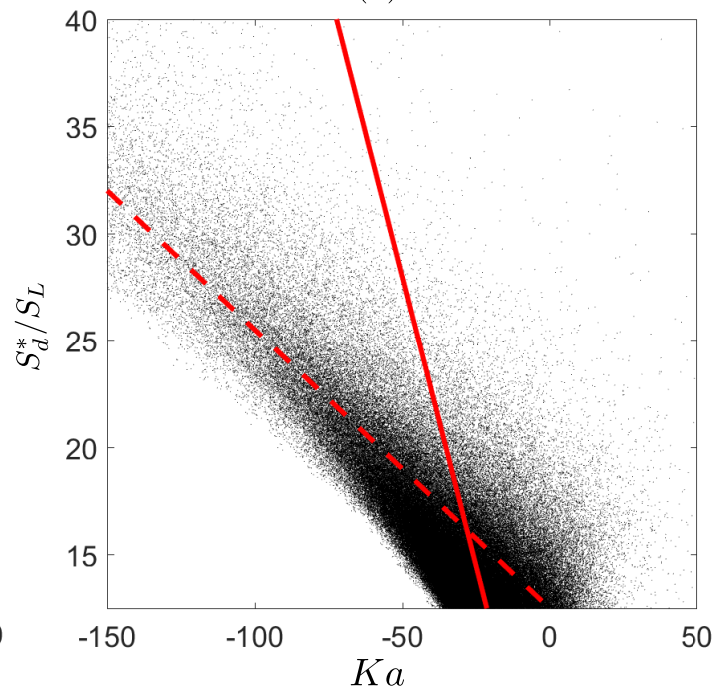

Figure 6.12: Correlation between $K a$ and $S_{d}^{*} / S_{L}$ for annihilation events from DNS (scatter), original theory (equation 6.9) (solid line) and new proposed linear model (equation 6.11) (dashed line): (a) $\phi=1.0$ and (b) $\phi=0.7$.

\subsection{Contributions of Different Terms of Flame Displacement Speed to Noise Generation by Turbulent Premixed Flames}

Here, the contribution of different $S_{d}^{*} / S_{L}$ terms to the sound produced by annihilation events is evaluated through the simple model proposed in chapter 5 .

Figure 6.13 depicts the time history of the acoustic pressure $p^{\prime}$ at $x=10 D$ and $r=6 D$ obtained from the simple model (equation 5.6) for the case with $\phi=1.0$. It is observed that the reaction term plays the most significant role, followed by the curvature term and, lastly, the normal diffusion term. The same order of contributions was also observed for the lean flame (not shown here). The reaction rate effect during flame annihilation events therefore outweighs the curvature term for the flames studied here.

Figure 6.13b compares the results of the simple model with $V_{f}=\mathbf{u . n}+S_{d}$ and that with considering only the contribution from the reaction term $V_{f}=\mathbf{u . n}+S_{d_{r}}$. As can be seen, there is a very good agreement between both predictions. This suggests that the knowledge of the reaction term and gas velocity should suffice 

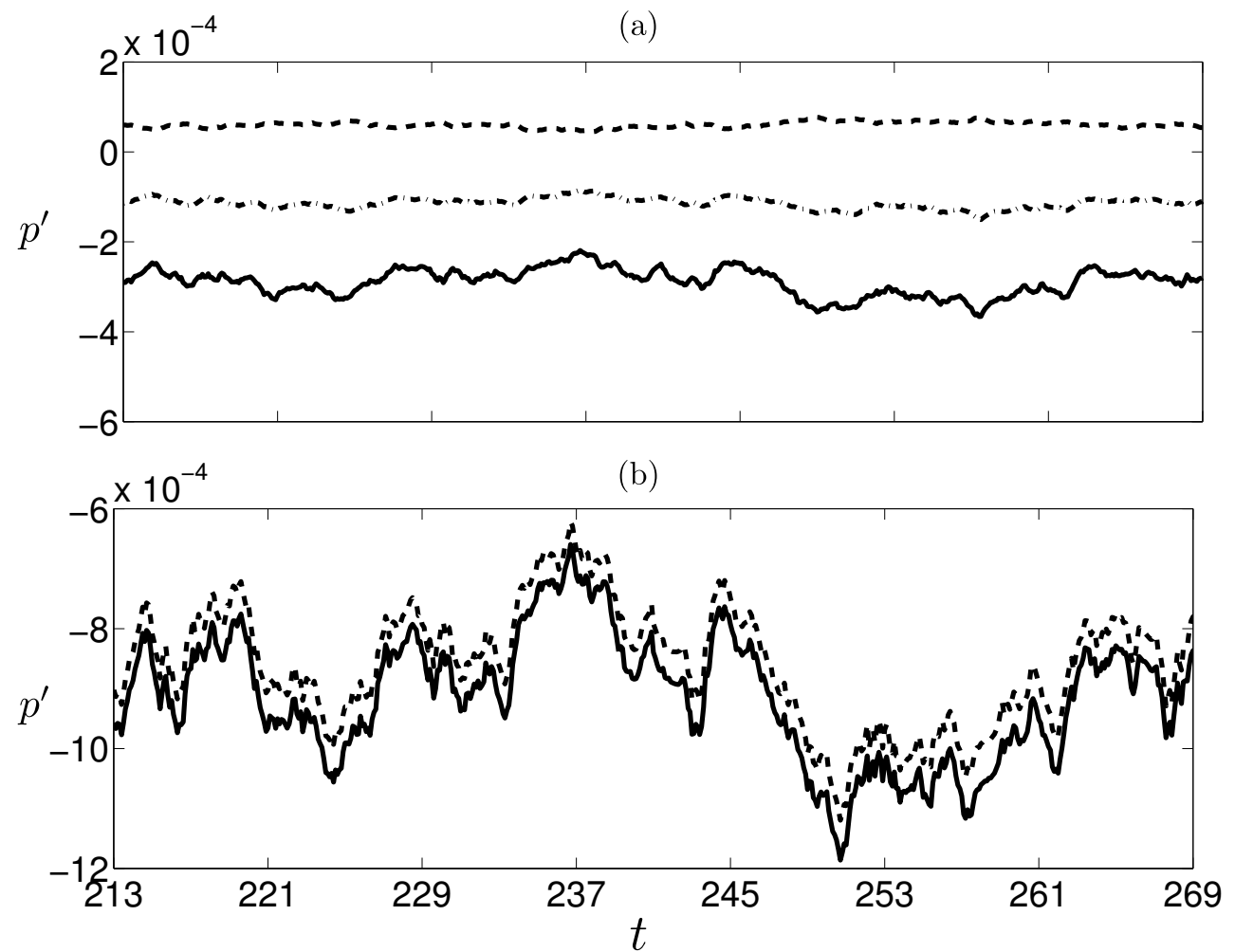

Figure 6.13: Time history of the far-field noise at $r=6 D$ and $x=10 D$ for $\phi=1.0$, obtained from the simple model proposed in chapter 5 with different terms of $S_{d}^{*} / S_{L}$ contributing to $V_{f}$. (a) Excluding the gas velocity term (u.n): reaction term (solid), normal diffusion term (dashed) and curvature term (dash-dot).

(b) Including the gas velocity term (u.n): net term (solid) and reaction term (dashed).

for prediction of the noise generated by annihilation events when the simple model is used.

\subsection{A Revised Simple Model of Noise Genera- tion by Turbulent Premixed Flames}

A model for the response of turbulent premixed flames to stretch for flame annihilation events has already been proposed: $S_{d}^{*} / S_{L}=n-M a^{*} K a$. The validity of this linear theory will be assessed here by predicting the far-field noise using the simple model proposed in Chapter 5. Figure 6.14 compares the time history of 
(a)

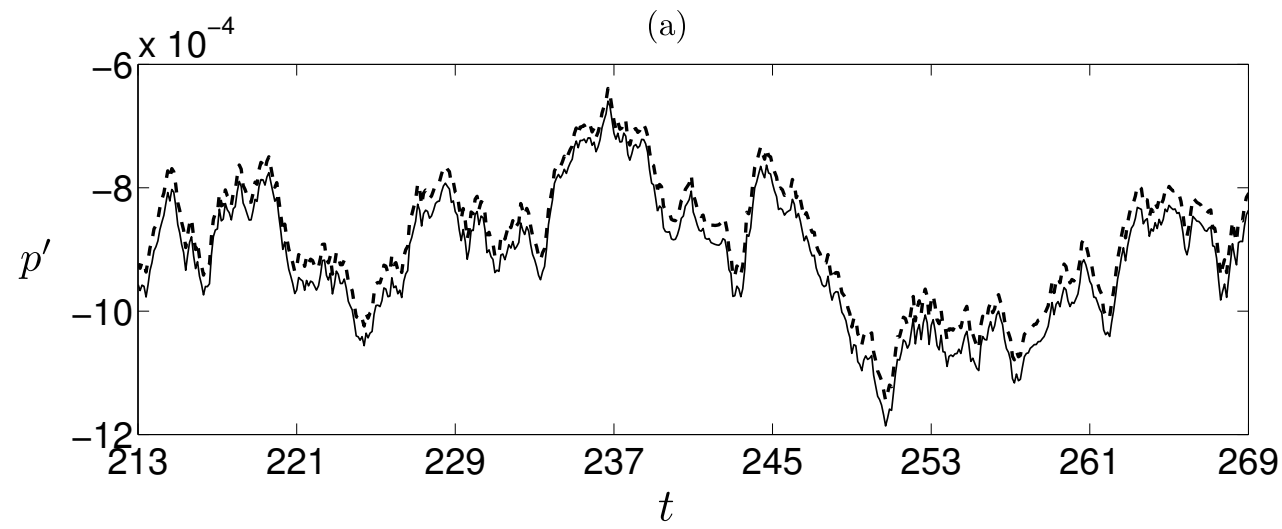

(b)

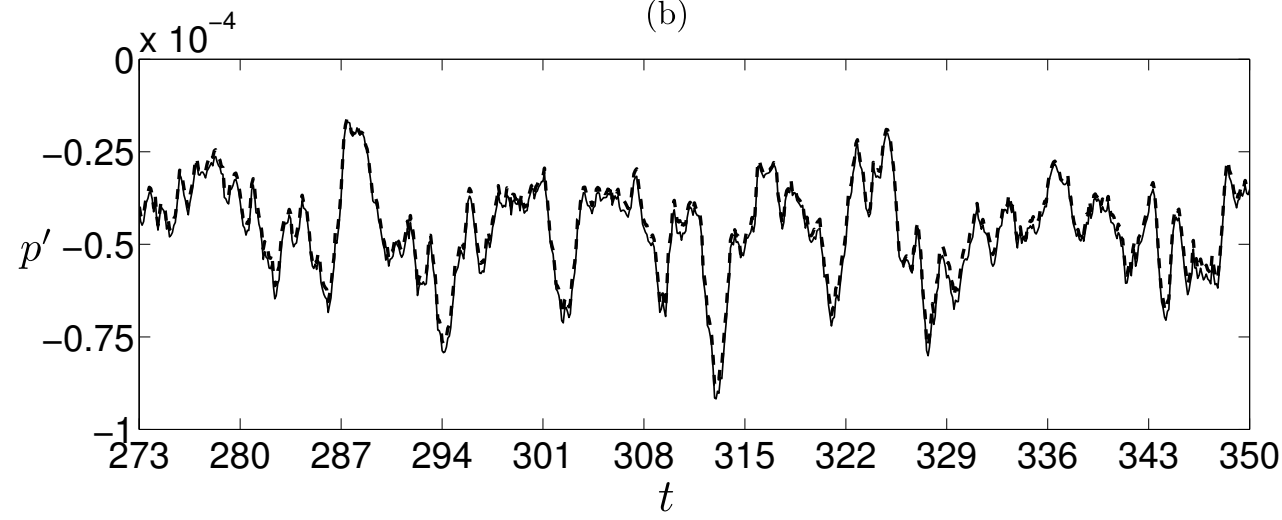

Figure 6.14: Time history of the far-field noise at $r=6 D$ and $x=10 D$ obtained from our simple model with $V_{f}=\mathbf{u . n}+S_{d_{\mathrm{DNS}}}($ solid $)$ and with $V_{f}=\mathbf{u . n}+S_{d_{\text {model }}}$ (dashed) for (a) $\phi=1.0$ and (b) $\phi=0.7$.

the far-field noise at $r=6 D$ and $x=10 D$ obtained from the simple model with $S_{d}$ from the DNS and also from the new proposed linear model for both flames. A very good agreement between both predictions confirms that equation 6.11 can be used over a broad range of $K a$ during flame annihilation. This observation therefore indicates that this new model can reasonably predict the flame displacement speed, and can be successfully incorporated into the simple model proposed in Chapter 5 for prediction of the sound produced by annihilation events. 


\subsection{Conclusions}

This chapter presented a statistical analysis of flame annihilation events in order to examine the flame response to strain and curvature. The following key observations were made.

- It was shown that annihilation events generally feature high negative values of curvature, consistent with the qualitative observations in Chapter 4. Analysis of different terms contributing to the displacement speed during flame annihilation also showed that the reaction term had the highest contribution, followed by the curvature term and with minimal contribution from the normal diffusion term. Furthermore, a strong correlation between the flame displacement speed and curvature was observed. It was found that the reaction term featured a stronger correlation with curvature compared with the diffusion term.

- The normalised flame stretch $(K a)$ with the contributions from tangential strain rate $\left(K a_{S}\right)$ and curvature $\left(K a_{C}\right)$ was also analysed. It was observed that the curvature component of stretch is the dominant term during annihilation, indicating that destruction of flame surface area is the dominant phenomenon during annihilation. Moreover, a stronger correlation (more than $80 \%$ ) between the flame stretch and the flame displacement speed was observed during flame annihilation compared to that of the entire flame surface.

- A modified form of the Markstein's linear theory for estimation of the flame displacement speed during flame annihilation was proposed. The Markstein number $M a$ was calculated using a least-squares linear fit over the DNS data. Finally, it was shown that this new model can be successfully used in the simple model proposed in Chapter 5 for prediction of the sound produced by annihilation events. 


\section{Chapter 7}

\section{Conclusions and Recommendations}

A numerical and theoretical study of the sound generated by turbulent, premixed flames was presented. Direct numerical simulations (DNS) of two round jet methane/air flames with equivalence ratios of 0.7 and 1.0 were first carried out. Single step chemistry was employed to reduce the computational cost, and care was taken to resolve both the near and far fields and to avoid spurious noise reflections at the outflow boundaries. The simulations were performed using a constant time step for 6 jet flow-through times $\tau_{j}=L_{x} / U_{j}$, and only the data from the last $4 \tau_{j}$ were used for post-processing. Overall, the computational costs for the stoichiometric and lean cases were equal to 1.4 and 1.9 million CPUhours, respectively, running on 9216 computing cores (Intel Xeon E5-2690V3 "Haswell" processors, $2.6 \mathrm{GHz}$ ).

Chapters 3 and 4 provided the DNS setup and the key DNS results, respectively, with significant role of flame annihilation events observed in the sound produced. A means of identifying the observed annihilation events was then defined in Chapter 5, and a simple model that related these events to the far-field sound was also proposed. Finally, a statistical analysis of the identified flame annihilation events in response to strain and curvature was presented in Chapter 6.

The conclusions drawn from these studies are given as follows.

1) The stoichiometric flame was significantly louder than the lean 
flame, with the observed frequency of peak acoustic emission being consistent with prior experimental studies. Furthermore, annihilation events were found to be significant acoustic sources.

Several significant features of the two simulated flames were shown. First, the acoustic fields of both the stoichiometric and lean flames exhibited monopolar features that originated within the flame, with the stoichiometric flame being significantly louder than the lean flame. These observations are consistent with several experimental studies of turbulent jet flames in the literature. Further, socalled 'flame annihilation' events were observed to be a source of these monopolar, acoustic features, which was consistent with prior works on laminar premixed flames.

Three types of flame annihilation events were observed:

- 'island burn-out'

This event involves consumption of a pocket of unburned gases, particularly in the downstream region near the flame tip.

- 'pinch-off'

This events occurs where a pocket of unburned gases is locally detached from the flame surface.

- 'tunnel formation'

This event takes place when flame-to-flame interaction results in the formation of a tunnel of burned gas. The local flame area is then lost due to cusp retraction associated with another 'pinch-off' event.

The acoustic spectra of both flames were also studied. These were found to be broadband in nature, with a frequency at the peak spectral amplitude agreeing with that observed experimentally by Rajaram \& Lieuwen [2009].

2) A simple model, relating the annihilation events to the far-field sound, demonstrated the significance of these events to the produced sound, in particular for the stoichiometric flame. 
A simple model that related the observed annihilation events to the far-field sound was proposed. This model considered the far-field sound as the superposition of the sound radiated by individual, spherically symmetric annihilation events that occurred throughout the flame. These events were identified by conditioning the flame displacement speed at some threshold since flame annihilation is well known to feature flame acceleration. The choice of threshold was shown to strongly affect the correlation between the modelled and measured, far-field sound, with the correlation coefficient exceeding 0.5 in some cases. Such levels of correlation were surprising given the model's simplicity, and demonstrated the importance of flame annihilation as acoustic sources in turbulent premixed flames. This model is also consistent with Smith \& Kilham's 1963 model of a flame as a distribution of monopolar sound sources, with the present work identifying flame annihilation events as these sources and, in so doing, providing a physical explanation for the well known, monopolar content of the sound radiated by turbulent premixed flames.

3) Flame curvature plays a significance role during annihilation. Further, a modified form of Markstein's linear theory was proposed for estimation of the flame displacement speed during flame annihilation. This model was successfully used within the simple sound model previously proposed for calculation of sound generated by annihilation events.

A detailed analysis of annihilation events in terms of their response to strain and curvature was performed. Analysis of different terms contributing to the flame displacement speed showed that the reaction term had the highest contribution to the flame displacement speed, followed by the curvature term and with minimal contribution from the normal diffusion term during flame annihilation. Further, a strong correlation between the flame displacement speed and curvature was also observed, with the annihilation events featuring highly curved flame surfaces towards reactant (negative values of curvature).

The contribution of curvature term $\left(K a_{C}\right)$ to the normalised flame stretch $(K a)$ was significantly higher than that of the tangential strain rate term $\left(K a_{S}\right)$ for the annihilation events, indicating the predominance of flame surface destruc- 
tion. However, completely opposite behaviour was observed when the entire flame surface was analysed. Moreover, a stronger correlation (more than $80 \%$ ) between the flame stretch and the flame displacement speed during flame annihilation was observed compared to that of the entire flame surface statistics. Motivated by this observation, a modified form of the Markstein's linear theory for estimation of the flame displacement speed during flame annihilation was proposed. The Markstein number $M a$ was calculated using a least-squares linear fit over the DNS data, showing a smaller value for annihilation events compared with other parts of the flame surface. Finally, it was shown that this new model can be successfully used in the simple model proposed in Chapter 5 for prediction of the sound produced by annihilation events.

\subsection{Recommendations for Future Research}

\section{1) Identifying different types of flame annihilation events within these simulations and determining their relative contributions to the sound produced by turbulent premixed flames.}

It has been shown in the present study that annihilation events are important sources of sound in turbulent premixed flames. However, different topologies of annihilation events are not studied here. A means of identifying different types of flame annihilation events within these simulations should therefore be defined. Development of an automatic method is recommended to identify the types of flame interactions such as island burn-out, pinch-off and tunnel formation in the current DNS dataset. As such, the behaviour of these different types of annihilation events can be examined and their relative contributions to the overall sound produced can be clarified.

2) Performing Large Eddy Simulations (LES) of the present turbulent premixed flames and evaluating the validity of LES models for combustion noise studies.

It was shown that several studies (see Chapter 2) on combustion noise have 
used LES, combined with acoustic analogies to examine sound generation by turbulent flames. The validity of their LES methods have been evaluated with experimental data by comparing the rms velocities or sound spectra. However, given the present DNS dataset, it will be useful to perform LES of the DNS cases to gain further insight about the validity of different combustion models in the prediction of sound. The results of this study will be useful for developing accurate LES models which can be used to simulate turbulent flames with higher Reynolds numbers that are of more practical interest.

\section{3) Examining the contribution of the source terms of Dowling's formu- lation to the produced sound by turbulent premixed flames.}

The work by Talei et al. [2014] shows that several acoustic source terms that are not directly related to the unsteady heat release may also be significant in sound generation by premixed flames. However, this earlier work only considered laminar flames. The present dataset provides an opportunity to examine this hypothesis for turbulent premixed flames. This has important implications for turbulence modelling particularly RANS and LES. 


\section{Bibliography}

Balachandran, R., Ayoola, B. O., Kaminski, C. F., Dowling, A. P. \& Mastorakos, E. 2005 Experimental investigation of the nonlinear response of turbulent premixed flames to imposed inlet velocity oscillations. Combustion and Flame 143 (1), 37-55.

Bechtold, JK \& Matalon, M 2001 The dependence of the markstein length on stoichiometry. Combustion and Flame 127 (1), 1906-1913.

Birbaud, A. L., Ducruix, S., Durox, D. \& Candel, S. 2006 Dynamics of free jets modulated by plane acoustic waves: Part 2 . numerical simulations. In 12 th AIAA/CEAS Aeroacoustics Conference(27 th AIAA Aeroacoustics Conference).

Bodony, D. J. \& Lele, S. K. 2005 On using large-eddy simulation for the prediction of noise from cold and heated turbulent jets. Physics of Fluids 17 (8), $1-20$.

Bogey, C, Bailly, C \& Juvé, D 2000 Numerical simulation of sound generated by vortex pairing in a mixing layer. AIAA J. 38 (12), 2210-2218.

BorGHI, R 1985 On the structure and morphology of turbulent premixed flames. In Recent advances in the Aerospace Sciences, pp. 117-138. Springer.

Bragg, S. L. 1963 Combustion noise. Journal of the Institute of Fuel 36 (1), $12-16$.

Brear, M. J., Nicoud, F., Talei, M., Giauque, A. \& Hawkes, E. R. 2012 Disturbance energy transport and sound production in gaseous combustion. Journal of Fluid Mechanics 1 (1), 1-21. 
Briffa, F. J., Clark, C. J. \& Williams, G. T. 1973 Combustion noise. Journal of the Institute of Fuel 46, 207-216.

Bui, T. P., Ihme, M., SchröDer, W. \& Pitsch, H. 2009 Analysis of different sound source formulations to simulate combustion generated noise using a hybrid LES/APE-RF method. International Journal of Aeroacoustics 8 (1), 95-123.

Bui, T. P., Meinke, M., Schröder, W., Flemming, F., Sadiki, A. \& JANICKA, J. 2005 A hybrid method for combustion noise based on LES and APE. AIAA Paper 3014, 2005.

Bui, T. P., Schröder, W. \& Meinke, M. 2008 Numerical analysis of the acoustic field of reacting flows via acoustic perturbation equations. Computers \& Fluids 37 (9), 1157-1169.

Candel, S, Durox, D \& Schuller, T 2004 Flame interactions as a source of noise and combustion instabilities. In Collection of Technical Papers-10th AIAA/CEAS Aeroacoustics Conference.

Candel, S.M. \& Poinsot, T. 1990 Flame stretch and the balance equation for the flame area. Combustion Science and Technology 70 (1-3), 1-15.

Chakraborty, N. \& Cant, R.S. 2005 Influence of lewis number on curvature effects in turbulent premixed flame propagation in the thin reaction zones regime. Physics of Fluids 17 (10), 105105.

Chakraborty, N. \& Cant, R. S. 2009 Direct numerical simulation analysis of the flame surface density transport equation in the context of large eddy simulation. Proceedings of the Combustion Institute 32 (1), 1445-1453.

Chakraborty, N. \& Cant, S. 2004 Unsteady effects of strain rate and curvature on turbulent premixed flames in an inflow-outflow configuration. Combustion and Flame 137 (1), 129-147.

Chakraborty, N, Hesse, H \& Mastorakos, E 2010 Numerical investigation of edge flame propagation behavior in an igniting turbulent planar jet. Combustion Science and Technology 182 (11-12), 1747-1781. 
Chakraborty, N., Konstantinou, I. \& Lipatnikov, A. 2016 Effects of Lewis number on vorticity and enstrophy transport in turbulent premixed flames. Physics of Fluids 28 (1), 015109.

Chakraborty, N. \& Mastorakos, E. 2006 Numerical investigation of edge flame propagation characteristics in turbulent mixing layers. Physics of Fluids 18 (10), 105103.

Chakraborty, N. \& Mastorakos, E 2008 Direct numerical simulations of localised forced ignition in turbulent mixing layers: the effects of mixture fraction and its gradient. Flow, Turbulence and Combustion 80 (2), 155-186.

Chen, J.B. \& Im, H.G. 2000 Stretch effects on the burning velocity of turbulent premixed hydrogen/air flames. Proceedings of the Combustion Institute 28 (1), $211-218$.

Chen, J. H., Choudhary, A., De Supinski, B., DeVries, M, Hawkes, E. R., Klasky, S., Liao, W. K., Ma, K. L., Mellor-Crummey, J., Podhorszki, N., Sankaran, R., Shende, S. \& Yoo, C. S. 2009 Terascale direct numerical simulations of turbulent combustion using S3D. Computational Science 6 Discovery 2 (1), 015001.

Chen, J H, Echekki, T \& Kollmann, W 1999 The mechanism of twodimensional pocket formation in lean premixed methane-air flames with implications to turbulent combustion. Combustion and Flame 116 (1), 15-48.

Chen, J H \& IM, H G 1998 Correlation of flame speed with stretch in turbulent premixed methane/air flames. In Symposium (International) on Combustion, , vol. 27, pp. 819-826. Elsevier.

Clavin, P \& Joulin, G 1983 Premixed flames in large scale and high intensity turbulent flow. Journal de Physique Lettres 44 (1), 1-12.

Clavin, P. \& Siggia, E.D. 1991 Turbulent premixed flames and sound generation. Combust Sci. Technol. 78 (1-3), 147-155.

Colonius, T., Lele, S. K. \& Moin, P. 1997 Sound generation in a mixing layer. Journal of Fluid Mechanics 330, 375-409. 
DoAK, P.E. 1972 Analysis of internally generated sound in continuous materials: A critical review of the conceptual adequacy and physical scope of existing theories of aerodynamic noise, with special reference to supersonic jet noise. Journal of Sound and Vibration 25 (2), 263-335.

Dowling, A.P. 1992 Modern Methods in Analytical Acoustics, Chapter 13: Thermoacoustic sources and instabilities, pp. 378-403. Springer.

Dowling, A. P. \& Williams, J. E . F. 1983 Sound and sources of sound..

Duffy, D.G. 2001 Green's functions with applications. Chapman \& Hall/CRC.

Dunstan, T. D., Swaminathan, N., Bray, K. N. C. \& Kingsbury, N. G. 2013 Flame interactions in turbulent premixed twin V-flames. Combust. Sci. and Technol. 185 (1), 134-159.

Durox, D, Ducruix, S. \& CAndel, S. 2001 Experiments on collapsing cylindrical flames. Combustion and Flame 125 (1), 982-1000.

Echekki, TARek \& Chen, Jacqueline H 1996 Unsteady strain rate and curvature effects in turbulent premixed methane-air flames. Combustion and Flame 106 (1), 184-202.

Echekki, TAReK \& Chen, Jacqueline H 1999 Analysis of the contribution of curvature to premixed flame propagation. Combustion and Flame 118 (1), 308-311.

Echekki, T., Chen, J. H. \& Gran, I. 1996 The mechanism of mutual annihilation of stoichiometric premixed methane-air flames. Symposium (International) on Combustion 26 (1), 855 - 863.

Flemming, F., SAdiki, A. \& JanickA, J. 2007 Investigation of combustion noise using a LES/CAA hybrid approach. Proceedings of the Combustion Institute 31 II, 3189-3196.

Fogla, N., Creta, F. \& Matalon, M. 2015 Effect of folds and pockets on the topology and propagation of premixed turbulent flames. Combustion and Flame 162 (7), 2758-2777. 
Griffiths, R. A. C., Chen, J. H., Kolla, H., Cant, R. S. \& Kollmann, W. 2015 Three-dimensional topology of turbulent premixed flame interaction. Proceedings of the Combustion Institute 35 (2), 1341-1348.

Gülder, Ö \& Smallwood, G J 2007 Flame surface densities in premixed combustion at medium to high turbulence intensities. Combustion Science and Technology 179 (1-2), 191-206.

Haq, MZ, Sheppard, CGW, Woolley, R, Greenhalgh, DA \& Lockett, RD 2002 Wrinkling and curvature of laminar and turbulent premixed flames. Combustion and Flame 131 (1), 1-15.

Hassan, H. A . 1974 Scaling of combustion-generated noise. Journal of Fluid Mechanics 66 (Part 3), 445-453.

Hawkes, E R \& Chen, J H 2004 Direct numerical simulation of hydrogenenriched lean premixed methane-air flames. Combustion and Flame 138 (3), 242-258.

Hawkes, E R \& Chen, J H 2005 Evaluation of models for flame stretch due to curvature in the thin reaction zones regime. Proceedings of the Combustion Institute 30 (1), 647-655.

Hawkes, E R \& Chen, J H 2006 Comparison of direct numerical simulation of lean premixed methane-air flames with strained laminar flame calculations. Combustion and Flame 144 (1), 112-125.

Haworth, D. C. \& Poinsot, T. J. 1992 Numerical simulations of lewis number effects in turbulent premixed flames. J. Fluid Mech. 244, 405-436.

Hurle, I. R., Price, R. B., Sugden, T. M. \& Thomas, A. 1968 Sound emission from open turbulent premixed flames. Proceedings of the Royal Society of London. Series A. Mathematical and Physical Sciences 303 (1475), 409-427.

Hussein, H. J., Capp, S. P. \& George, W. K. 1994 Velocity measurements in a high-reynolds-number, momentum-conserving, axisymmetric, turbulent jet. Journal of Fluid Mechanics 258, 31-75. 
Ihme, M. \& Pitsch, H. 2012 On the generation of direct combustion noise in turbulent non-premixed flames. International Journal of Aeroacoustics. 11 (1), $25-78$.

Ihme, M., Pitsch, H. \& Bodony, D. 2009 Radiation of noise in turbulent non-premixed flames. Proceedings of the Combustion Institute 32 (1), 15451553.

Jiménez, C., Haghiri, A., Talei, M., Brear, M. J. \& Hawkes, E. R. 2015 Sound generation by premixed flame annihilation with full and simple chemistry. Proceedings of the Combustion Institute 35, 3317-3325.

Karami, S., Hawkes, E. R., Talei, M. \& Chen, J. H. 2015 Mechanisms of flame stabilisation at low lifted height in a turbulent lifted slot-jet flame. Journal of Fluid Mechanics 777, 633-689.

Karami, S., Hawkes, E. R., Talei, M. \& Chen, J. H. 2016 a Edge flame structure in a turbulent lifted flame: A direct numerical simulation study. Combustion and Flame 169, 110-128.

Karami, S., Talei, M., Hawkes, E. R. \& Chen, J. H $2016 b$ Local extinction and reignition mechanism in a turbulent lifted flame: A direct numerical simulation study. Proceedings of the Combustion Institute .

Kee, R. J., Grcar, J. F., Smooke, M. D., Miller, J. A. \& Meeks, E. 1985 Premix: a fortran program for modeling steady laminar one-dimensional premixed flames. Sandia National Laboratories Report .

Kennedy, C. A., Carpenter, M. H. \& Lewis, R. M. 2000 Low-storage, explicit runge-kutta schemes for the compressible navier-stokes equations. Applied Numerical Mathematics 35 (3), 177-219.

Kerl, Johannes, Lawn, Chris \& Beyrau, Frank 2013 Three-dimensional flame displacement speed and flame front curvature measurements using quadplane piv. Combustion and Flame 160 (12), 2757-2769. 
Kidin, N., Librovich, V., MacQuisten, M., Roberts, J. \& VuillerMOz, M. 1988 Possible acoustic source in turbulent combustion. Dynamics of Reactive Systems: Part 1: Flames pp. 336-348.

Kidin, N., Librovich, V., Roberts, J. \& Vuillermoz, M. 1984 On sound sources in turbulent combustion. Progress in Astronautics and Aeronautics 95, 343-355.

Kostiuk, LW \& BRAy, KNC 1993 Mean effects of stretch on laminar flamelets in a premixed turbulent flame. Combustion Science and Technology 95 (1-6), $193-212$.

Kotake, S. \& Hatta, K. 1965 On the noise of diffusion flames. Bulletin of JSME 8 (30), 211-219.

Kotake, S. \& Takamoto, K. 1990 Combustion noise: effects of the velocity turbulence of unburned mixture. Journal of Sound and Vibration 139 (1), 9-20.

LAW, C.K. 2006 Combustion physics. Cambridge University Press.

LAW, CK \& Sung, CJ 2000 Structure, aerodynamics, and geometry of premixed flamelets. Progress in Energy and Combustion Science 26 (4), 459-505.

LibBy, P. A.And Williams, F. A. 1994 Turbulent reacting flows. Academic press London.

Lighthill, M. J. 1952 On sound generated aerodynamically. i. general theory. Proc. R. Soc. Lond. A 211, 564-587.

Lighthill, M. J. 1954 On sound generated aerodynamically. ii. turbulence as a source of sound. Proc. R. Soc. Lond. A 222 (1148), 1-32.

LiU, Y. 2015 Two-time correlation of heat release rate and spectrum of combustion noise from turbulent premixed flames. Journal of Sound and Vibration 353, 119-134.

LockARD, D. P. 2000 An efficient, two-dimensional implementation of the ffowcs williams and hawkings equation. Journal of Sound and Vibration 229 (4), 897911. 
Lodato, G., Domingo, P. \& Vervisch, L. 2008 Three-dimensional boundary conditions for direct and large-eddy simulation of compressible viscous flows. Journal of Computational Physics 227 (10), 5105-5143.

LuO, KH 1999 Combustion effects on turbulence in a partially premixed supersonic diffusion flame. Combustion and Flame 119 (4), 417-435.

Ma, Lin, Wu, Yue, Lei, Qingchun, Xu, Wenjiang \& Carter, CampBELL D 2016 3D flame topography and curvature measurements at $5 \mathrm{khz}$ on a premixed turbulent bunsen flame. Combustion and Flame 166, 66-75.

Markstein, G.H. 1964 Non-steady flame propagation. Pergamon. New York.

Matalon, M. 1983 On flame stretch. Combustion Science and Technology 31 (34), 169-181.

Matalon, M. \& Matkowsky, B.J. 1982 Flames as gasdynamic discontinuities. Journal of Fluid Mechanics 124, 239-259.

Mehravaran, K \& Jaberi, FA 2004 Direct numerical simulation of transitional and turbulent buoyant planar jet flames. Physics of Fluids 16 (12), 4443-4461.

Najm, H.N. \& WyCKOFF, P.S. 1997 Premixed flame response to unsteady strain rate and curvature. Combustion and Flame 110 (1), 92-112.

Nishiki, S, Hasegawa, T, Borghi, R \& Himeno, R 2006 Modelling of turbulent scalar flux in turbulent premixed flames based on dns databases. Combustion Theory and Modelling 10 (1), 39-55.

O'Connor, J., Acharya, V. \& Lieuwen, T 2015 Transverse combustion instabilities: Acoustic, fluid mechanic, and flame processes. Progress in Energy and Combustion Science 49, 1-39.

Passot, T. \& Pouquet, A. 1987 Numerical simulation of compressible homogeneous flows in the turbulent regime. Journal of Fluid Mechanics 181, $441-466$. 
Peters, N. 2000 Turbulent combustion. Cambridge university press.

Peters, N, Terhoeven, P, Chen, Jacqueline H \& Echekki, Tarek 1998 Statistics of flame displacement speeds from computations of 2-d unsteady methane-air flames. In Symposium (International) on Combustion, , vol. 27, pp. 833-839. Elsevier.

Poinsot, T., Echekki, T. \& Mungal, M. G. 1992 A study of the laminar flame tip and implications for premixed turbulent combustion. Combustion Science and Technology 81 (1-3), 45-73.

Poinsot, T.J. \& Lele, S. K. 1992 Boundary conditions for direct simulations of compressible viscous flows. Journal of Computational Physics 101 (1), 104129.

Poinsot, T. \& Veynante, D. 2005 Theoretical and Numerical Combustion. RT Edwards Inc.

Pope, SB 1988 The evolution of surfaces in turbulence. International Journal of Engineering Science 26 (5), 445-469.

Price, RB, Hurle, IR \& Sugden, TM 1969 Optical studies of the generation of noise in turbulent flames. Symposium (International) on Combustion 12 (1), 1093-1102.

RAJARAm, R. 2007 Characteristics of sound radiation from turbulent premixed flames. PhD thesis, Georgia Institute of Technology.

Rajaram, R., Gray, J. \& Lieuwen, T. 2006 Premixed combustion noise scaling: Total power and spectra. AIAA Paper 2006-2612.

Rajaram, R. \& Lieuwen, T. 2003 Parametric studies of acoustic radiation from premixed flames. Combustion Science and Technology 175 (12), 2269 2298.

Rajaram, R. \& Lieuwen, T. 2009 Acoustic radiation from turbulent premixed flames. Journal of Fluid Mechanics 637, 357-385. 
Sankaran, R., Hawkes, E. R., Yoo, C. S. \& Chen, J. H. 2015 Response of flame thickness and propagation speed under intense turbulence in spatially developing lean premixed methane-air jet flames. Combustion and Flame 162 (9), 3294-3306.

Schlimpert, S, Koh, SR, Pausch, K, Meinke, M \& Schröder, W 2017 Analysis of combustion noise of a turbulent premixed slot jet flame. Combustion and Flame 175, 292-306.

Schuller, T., Durox, D. \& Candel, S. 2003 Self-induced combustion oscillations of laminar premixed flames stabilized on annular burners. Combustion and Flame 135 (4), 525-537.

SeArby, G \& Quinard, J 1990 Direct and indirect measurements of markstein numbers of premixed flames. Combustion and Flame 82 (3-4), 298-311.

Silva, C. F., Leyko, M., Nicoud, F. \& Moreau, S. 2013 Assessment of combustion noise in a premixed swirled combustor via large-eddy simulation. Computers \& Fluids 78, 1-9.

Sinibaldi, J O, Driscoll, J F, Mueller, C J, Donbar, J M \& Carter, C D 2003 Propagation speeds and stretch rates measured along wrinkled flames to assess the theory of flame stretch. Combustion and Flame 133 (3), 323-334.

Smith, T. J. B. \& Kilham, J. K. 1963 Noise generation by open turbulent flames. The Journal of the Acoustical Society of America 35, 715.

Smithson, R. N. \& Foster, P. J. 1965 Combustion noise from a meker burner. Combustion and Flame 9 (4), 426-428.

Strahle, W.C. 1971 On combustion generated noise. Journal of Fluid Mechanics 49 (2), 399-414.

Strahle, W. C. 1972 Some results in combustion generated noise. Journal of Sound and Vibration 23 (1), 113 - 125.

Strahle, W. C. 1978 Combustion noise. Progress in Energy and Combustion Science 4 (3), 157 - 176. 
Strahle, W. C. 1985 A More Modern Theory of Combustion Noise.. Plenum Press, New York, NY.

Strahle, W. C. \& Shivashankara, B. N. 1975 A rational correlation of combustion noise results from open turbulent premixed flames. Symposium (International) on Combustion 15 (1), 1379 - 1385.

Sun, C. J. \& LAw, C. K. 1998 On the consumption of fuel pockets via inwardly propagating flames. Symposium (International) on Combustion 27 (1), 963970.

Sutherland, J C \& Kennedy, C A 2003 Improved boundary conditions for viscous, reacting, compressible flows. Journal of Computational Physics 191 (2), 502-524.

Swaminathan, N., Balachandran, R., Xu, G. \& Dowling, A. P. $2011 a$ On the correlation of heat release rate in turbulent premixed flames. Proceedings of the Combustion Institute 33 (1), 1533-1541.

Swaminathan, N. \& Bray, K. N. C. 2011 Turbulent premixed flames. Cambridge University Press.

Swaminathan, N., Xu, G., Dowling, A. P. \& Balachandran, R. $2011 b$ Heat release rate correlation and combustion noise in premixed flames. Journal of Fluid Mechanics 681, 80-115.

Talei, M., Brear, M. J. \& Hawkes, E. R. 2011 Sound generation by laminar premixed flame annihilation. Journal of Fluid Mechanics 679, 194-218.

Talei, M., Brear, M. J. \& Hawkes, E. R. $2012 a$ A parametric study of sound generation by premixed laminar flame annihilation. Combustion and Flame 159 (2), 757-769.

Talei, M., Brear, M. J. \& Hawkes, E. R. 2014 A comparative study of sound generation by laminar, combusting and non-combusting jet flows. Theoretical and Computational Fluid Dynamics 28 (4), 385-408. 
Talei, M., Hawkes, E. R. \& Brear, M. J. $2012 b$ A direct numerical simulation study of frequency and Lewis number effects on sound generation by two-dimensional forced laminar premixed flames. Proceedings of the Combustion Institute 34, 1093-1100.

TAM, C. W. 2015 The spectral shape of combustion noise. International Journal of Aeroacoustics 14 (3-4), 431-456.

TAmadonfar, P \& Gülder, Ö 2015 Experimental investigation of the inner structure of premixed turbulent methane/air flames in the thin reaction zones regime. Combustion and Flame 162 (1), 115-128.

Thomas, A \& Williams, GT 1966 Flame noise: sound emission from sparkignited bubbles of combustible gas. Proceedings of the Royal Society of London. Series A. Mathematical and Physical Sciences 294 (1439), 449-466.

Thompson, K W 1990 Time-dependent boundary conditions for hyperbolic systems, ii. Journal of Computational Physics 89 (2), 439-461.

Turns, S. R. 2000 An Introduction to Combustion: Concepts and Application. Mc Graw Hill.

Wäsle, J., Winkler, A. \& Sattelmayer, T. 2005 Influence of the combustion mode on acoustic spectra of open turbulent swirl flames. In 12th International Congress on Sound and Vibration.

Westley, R \& Lilley, G Mo 1952 An investigation of the noise field from a small jet and methods for its reduction. College of Aeronautics, Cranfield. .

Wu, X. \& Moin, P. 2008 A direct numerical simulation study on the mean velocity characteristics in turbulent pipe flow. Journal of Fluid Mechanics 608, 81-112.

Yoo, C. S. \& Im, H. G. 2007 Characteristic boundary conditions for simulations of compressible reacting flows with multi-dimensional, viscous and reaction effects. Combustion Theory and Modelling 11 (2), 259-286. 
Yoo, C. S., Wang, Y., Trouvé, A. \& Im, H. G. 2005 Characteristic boundary conditions for direct simulations of turbulent counterflow flames. Combustion Theory and Modelling 9 (4), 617-646.

Zhang, F., Habisreuther, P., Bockhorn, H., Nawroth, H. \& PASChEREIT, C. 2013 On prediction of combustion generated noise with the turbulent heat release rate. Acta Acustica 99 (6), 940-951.

ZhaO, W. \& Frankel, S.H. 2001 Numerical simulations of sound radiated from an axisymmetric premixed reacting jet. Physics of Fluids 13 (9), 2671-2681. 


\section{Appendix A: Calculations and Derivations}

\section{A.1 Derivation of Lighthill's acoustic analogy}

In order to derive Lighthill's acoustic analogy, the continuity and momentum equations are first considered

$$
\begin{gathered}
\frac{\partial \rho}{\partial t}+\frac{\partial}{\partial x_{j}} \rho u_{j}=0 \\
\rho\left(\frac{\partial u_{i}}{\partial t}+u_{j} \frac{\partial}{\partial x_{j}} u_{i}\right)=-\frac{\partial p}{\partial x_{i}}+\frac{\partial \tau_{i j}}{\partial x_{j}} .
\end{gathered}
$$

where $\tau_{i j}$ is the viscous stress tensor given by,

$$
\tau_{i j}=\mu\left(\frac{\partial u_{i}}{\partial x_{j}}+\frac{\partial u_{j}}{\partial x_{i}}-\frac{2}{3} \delta_{i j} \frac{\partial u_{k}}{\partial x_{k}}\right),
$$

where $\mu$ is the viscosity of the fluid and $\delta_{i j}$ is the Kronecker $\delta$-function $\left(\delta_{i j}=1\right.$ if $\mathrm{i}=\mathrm{j}, \delta_{i j}=0$ if $\left.\mathrm{i} \neq \mathrm{j}\right)$. Multiplying the continuity equation 1 by $u_{i}$,

$$
u_{i} \frac{\partial \rho}{\partial t}+u_{i} \frac{\partial}{\partial x_{j}} \rho u_{j}=0
$$

and adding the result to the momentum equation 2 ,

$$
\rho \frac{\partial u_{i}}{\partial t}+\rho u_{j} \frac{\partial u_{i}}{\partial x_{j}}+u_{i} \frac{\partial \rho}{\partial t}+u_{i} \frac{\partial}{\partial x_{j}} \rho u_{j}=-\frac{\partial p}{\partial x_{i}}+\frac{\partial \tau_{i j}}{\partial x_{j}},
$$

the following equation results by combining the terms in equation 5 ;

$$
\frac{\partial}{\partial t}\left(\rho u_{i}\right)=-\frac{\partial}{\partial x_{j}}\left(\rho u_{i} u_{j}+\delta_{i j} p-\tau_{i j}\right)
$$


The above equation upon adding and subtracting the term $c_{0}^{2} \frac{\partial \rho}{\partial x_{i}}$ becomes,

$$
\frac{\partial}{\partial t}\left(\rho u_{i}\right)+c_{0}^{2} \frac{\partial \rho}{\partial x_{i}}=-\frac{\partial}{\partial x_{j}}\left(\rho u_{i} u_{j}+\delta_{i j} p-\tau_{i j}-c_{0}^{2} \rho \delta_{i j}\right) .
$$

Note that, the subscript 0 is used here to denote constant reference values. Since $p_{0}$ and $\rho_{0}$ are considered as some constant reference values, they can be expressed as follows:

$$
\frac{\partial}{\partial x_{j}} p=\frac{\partial}{\partial x_{j}}\left(p-p_{0}\right), \frac{\partial}{\partial x_{j}} \rho=\frac{\partial}{\partial x_{j}}\left(\rho-\rho_{0}\right) .
$$

Using these expression in equation 7 results in

$$
\frac{\partial}{\partial t}\left(\rho u_{i}\right)+c_{0}^{2} \frac{\partial \rho}{\partial x_{i}}=-\frac{\partial}{\partial x_{i}}\left(\rho u_{i} u_{j}+\delta_{i j}\left[\left(p-p_{0}\right)-c_{0}^{2}\left(\rho-\rho_{0}\right)\right]-\tau_{i j}\right)
$$

We can now differentiate equation 1 with respect to $t$ to get

$$
\frac{\partial^{2} \rho}{\partial t^{2}}+\frac{\partial}{\partial t \partial x_{j}} \rho u_{j}=0
$$

Taking the divergence of equation 9 .

$$
\frac{\partial^{2}}{\partial t \partial x_{j}}\left(\rho u_{i}\right)+c_{0}^{2} \frac{\partial^{2} \rho}{\partial x_{i} \partial x_{j}}=-\frac{\partial^{2}}{\partial x_{i} \partial x_{j}}\left(\rho u_{i} u_{j}+\delta_{i j}\left[\left(p-p_{0}\right)-c_{0}^{2}\left(\rho-\rho_{0}\right)\right]-\tau_{i j}\right)
$$

equation 8 allows us to write

$$
\frac{\partial^{2} \rho}{\partial t^{2}}=\frac{\partial^{2}\left(\rho_{0}+\rho^{\prime}\right)}{\partial t^{2}}=\frac{\partial^{2} \rho^{\prime}}{\partial t^{2}} \quad, \quad \frac{\partial^{2} \rho}{\partial x_{i} \partial x_{j}}=\frac{\partial^{2}\left(\rho_{0}+\rho^{\prime}\right)}{\partial x_{i} \partial x_{j}}=\frac{\partial^{2} \rho^{\prime}}{\partial x_{i} \partial x_{j}}
$$

By subtracting equation 11 from equation 10, Lighthill's inhomogeneous wave equation is obtained,

$$
\frac{\partial^{2} \rho^{\prime}}{\partial t^{2}}-c_{0}^{2} \nabla^{2} \rho^{\prime}=\frac{\partial^{2} T_{i j}}{\partial x_{i} \partial x_{j}}
$$


where

$$
T_{i j}=\rho u_{i} u_{j}+\delta_{i j}\left[\left(p-p_{0}\right)-c_{0}^{2}\left(\rho-\rho_{0}\right)\right]-\tau_{i j} .
$$

No approximation is made in this equation. The source of sound in Lighthill's acoustic analogy is a quadrupole distribution where the strength of the quadrupole per unit volume is Lighthill's stress tensor $T_{i j}$. That the source distribution is quadrupolar is determined by the double divergence structure of the right-hand side of equation 13 .

It is also possible to write Lighthill's wave equation 13 in a pressure form, using equation 13

$$
\frac{1}{c_{0}^{2}} \frac{\partial^{2} p}{\partial t^{2}}-\nabla^{2} p=\frac{\partial^{2}}{\partial x_{i} \partial x_{j}}\left(\rho u_{i} u_{j}-\tau_{i j}\right)+\frac{1}{c_{0}^{2}} \frac{\partial^{2}\left(p-p_{0}\right)}{\partial t^{2}}-\frac{\partial^{2}\left(\rho-\rho_{0}\right)}{\partial t^{2}},
$$

to give

$$
\frac{1}{c_{0}^{2}} \frac{\partial^{2} p}{\partial t^{2}}-\nabla^{2} p=\frac{\partial^{2}}{\partial x_{i} \partial x_{j}}\left(\rho u_{i} u_{j}-\tau_{i j}\right)-\frac{\partial^{2} \rho_{e}}{\partial t^{2}},
$$

where $\rho_{e}$ is the so-called "excess" density

$$
\rho_{e}=\rho-\rho_{0}-\left(p-p_{0}\right) / c_{0}^{2} .
$$

It is worth mentioning that $\rho_{e}$ vanishes where the entropy is uniform inside the source region.

\section{A.2 Derivation of Dowling's [1992] combusting form of Lighthill's acoustic analogy}

Lighthill's equation stated that

$$
\frac{\partial^{2} \rho^{\prime}}{\partial t^{2}}-c_{0}^{2} \nabla^{2} \rho^{\prime}=\frac{\partial^{2}}{\partial x_{i} \partial x_{j}} \rho u_{i} u_{j}+\delta_{i j}\left[\left(p-p_{0}\right)-c_{0}^{2}\left(\rho-\rho_{0}\right)\right]-\tau_{i j},
$$

where $\rho$ is the density, $p$ is the pressure, $\mathbf{u}$ is the particle velocity, $c$ is the sound speed and $\tau_{i j}$ is the viscous stress tensor. The suffix 0 denotes a mean value in 
the distant acoustic field. Equation 17 can be rearranged as follows:

$$
\frac{1}{c_{0}^{2}} \frac{\partial^{2} p}{\partial t^{2}}-\nabla^{2} p=\frac{\partial^{2}}{\partial x_{i} \partial x_{j}}\left(\rho u_{i} u_{j}-\tau_{i j}\right)+\frac{1}{c_{0}^{2}} \frac{\partial^{2}\left(p-p_{0}\right)}{\partial t^{2}}-\frac{\partial^{2}\left(\rho-\rho_{0}\right)}{\partial t^{2}},
$$

to give

$$
\frac{1}{c_{0}^{2}} \frac{\partial^{2} p}{\partial t^{2}}-\nabla^{2} p=\frac{\partial^{2}}{\partial x_{i} \partial x_{j}}\left(\rho u_{i} u_{j}-\tau_{i j}\right)-\frac{\partial^{2} \rho_{e}}{\partial t^{2}},
$$

Note that $\rho u_{i} u_{j}$ in equation 18 is the quadrupolar source term and the remaining source terms $\frac{\partial^{2}}{\partial x_{i} \partial x_{j}} \tau_{i j}$ and $\frac{\partial^{2} \rho_{e}}{\partial t^{2}}$ are "thermoacoustic" sources that are nonzero in regions of appreciable heat release. To determine the strength of these sources, the thermodynamics of the source region needs to be investigated. Hence, the energy conservation equation is considered

$$
\rho \frac{D e}{D t}=-\nabla \cdot \mathbf{q}-p \nabla \cdot \mathbf{u}+\frac{\partial u_{i}}{\partial x_{j}} \tau_{i j},
$$

where $e$ is the internal energy. For a gas made up of $N$ (possibly reacting) species we have

$$
d e=T d s+\frac{p}{\rho^{2}} d \rho+\sum_{n=1}^{N} \frac{\mu_{n}}{W_{n}} d Y_{n},
$$

where $s$ is the entropy, $\mu_{n}$ is the chemical potential of species $n$ and $W_{n}$ is the species molecular weight. Substituting for $e$ from equation 20 into equation 19 and using the continuity equation leads to an expression for entropy changes as follows:

$$
\rho T \frac{D s}{D t}=-\nabla \cdot \mathbf{q}+\frac{\partial u_{i}}{\partial x_{j}} \tau_{i j}-\sum_{n=1}^{N} \frac{\mu_{n}}{W_{n}} \rho \frac{D Y_{n}}{D t} .
$$

As for $p=p(\rho, s, Y)$ and from the chain rule

$$
\frac{D p}{D t}=c^{2} \frac{D \rho}{D t}+\left(\frac{\partial p}{\partial s}\right)_{\rho, Y_{n}} \frac{D s}{D t}+\sum_{n=1}^{N}\left(\frac{\partial p}{\partial Y_{n}}\right)_{s, \rho, Y_{m}} \frac{D Y_{n}}{D t} .
$$

Note that $\left(\frac{\partial p}{\partial \rho}\right)_{s, Y_{n}}=c^{2}$. When equation 22 is rearranged and equation 21 is used 
to replace $D s / D t$, we get

$$
\begin{aligned}
\frac{D \rho}{D t} & =\frac{1}{c^{2}} \frac{D p}{D t}+\left(\nabla \cdot \mathbf{q}-\frac{\partial u_{i}}{\partial x_{j}} \tau_{i j}\right) \frac{1}{\rho T c^{2}}\left(\frac{\partial p}{\partial s}\right)_{\rho, Y_{n}} \\
& +\sum_{n=1}^{N} \frac{1}{c^{2}}\left\{\frac{1}{T} \frac{\mu_{n}}{W_{n}}\left(\frac{\partial p}{\partial s}\right)_{\rho, Y_{n}}-\left(\frac{\partial p}{\partial Y_{n}}\right)_{s, \rho, Y_{m}}\right\} \frac{D Y_{n}}{D t} .
\end{aligned}
$$

It can be shown by repeated applications of the chain rule and using the enthalpy relationship,

$$
d h=T d s+\frac{1}{\rho} d p+\sum_{n=1}^{N} \frac{\mu_{n}}{W_{n}} d Y_{n}
$$

that

$$
\frac{1}{\rho T c^{2}}\left(\frac{\partial p}{\partial s}\right)_{\rho, Y_{n}}=\frac{\alpha}{c_{p}}
$$

and

$$
\frac{1}{c^{2}}\left\{\frac{1}{T} \frac{\mu_{n}}{W_{n}}\left(\frac{\partial p}{\partial s}\right)_{\rho, Y_{n}}-\left(\frac{\partial p}{\partial Y_{n}}\right)_{s, \rho, Y_{m}}\right\}=\frac{\rho \alpha}{c_{p}}\left(\frac{\partial h}{\partial Y_{n}}\right)_{\rho, p, Y_{m}} .
$$

Therefore equation 23 simplifies to

$$
\frac{D \rho}{D t}=\frac{1}{c^{2}} \frac{D p}{D t}+\frac{\alpha}{c_{p}}\left\{\sum_{n=1}^{N}\left(\frac{\partial h}{\partial Y_{n}}\right)_{\rho, p, Y_{m}} \rho \frac{D Y_{n}}{D t}+\nabla \cdot \mathbf{q}-\frac{\partial u_{i}}{\partial x_{j}} \tau_{i j}\right\},
$$

where $Y_{n}$ is the mass fraction of the $n$th species, $h$ is the enthalpy, $\mathbf{q}$ is the heat flux. In addition, $\alpha$ is the volumetric expansion coefficient and is equal to $T^{-1}$ for an ideal gas. Conservation of species can be expressed as

$$
\rho \frac{D Y_{n}}{D t}=\omega_{n}-\nabla . \mathbf{J}_{\mathbf{n}},
$$

where $\omega_{n}$ is the production rate per unite volume of species $n$ by reaction, and $\mathbf{J}_{\mathbf{n}}$ is the flux of species $n$ by diffusion. Equation 27 demonstrates that the density of a material particle changes not only due to pressure variations in a compressible fluid, but also because of the expansion caused by heating. 
Before the thermodynamic relationship in equation 27 can be used to substitute for $\frac{\partial^{2} \rho_{e}}{\partial t^{2}}$ in equation 18 , one of the partial time derivatives must be replaced by a material derivative. Straightforward algebraic manipulation leads to

$$
\frac{\partial \rho_{e}}{\partial t}=\frac{D \rho_{e}}{D t}-\frac{\rho_{e}}{\rho} \frac{D \rho}{D t}-\nabla \cdot\left(\mathbf{u} \rho_{e}\right)
$$

where the continuity equation has been used to replace $\nabla \cdot \mathbf{u}$ by $-\rho^{-1} D \rho / D t$. Once $\rho_{e}$ in equation 29 is written explicitly as $\rho-\rho_{0}-\left(p-p_{0}\right) / c_{0}^{2}$, equation 27 can be used to replace $D \rho / D t$. This leads to

$$
\begin{aligned}
\frac{\partial \rho_{e}}{\partial t} & =\frac{\alpha \rho_{0}}{c_{p} \rho}\left\{\sum_{n=1}^{N}\left(\frac{\partial h}{\partial Y_{n}}\right)_{\rho, p, Y_{m}} \rho \frac{D Y_{n}}{D t}+\nabla \cdot \mathbf{q}-\frac{\partial u_{i}}{\partial x_{j}} \tau_{i j}\right\}-\nabla \cdot\left(\mathbf{u} \rho_{e}\right) \\
& -\frac{1}{c_{0}^{2}}\left\{\left(1-\frac{\rho_{0} c_{0}^{2}}{\rho c^{2}}\right) \frac{D p}{D t}-\frac{\left(p-p_{0}\right)}{\rho} \frac{D \rho}{D t}\right\} .
\end{aligned}
$$

Finally, the inhomogeneous wave equation in equation 18 is accordingly equivalent to

$$
\begin{aligned}
\frac{1}{c_{0}^{2}} \frac{\partial^{2} p}{\partial t^{2}}-\nabla^{2} p & =-\frac{\partial}{\partial t}\left(\frac{\alpha \rho_{0}}{c_{p} \rho}\left\{\sum_{n=1}^{N}\left(\frac{\partial h}{\partial Y_{n}}\right)_{\rho, p, Y_{m}} \rho \frac{D Y_{n}}{D t}+\nabla \cdot \mathbf{q}-\frac{\partial u_{i}}{\partial x_{j}} \tau_{i j}\right\}\right) \\
& +\frac{\partial^{2}}{\partial x_{i} \partial x_{j}}\left(\rho u_{i} u_{j}-\tau_{i j}\right) \\
& +\frac{1}{c_{0}^{2}} \frac{\partial}{\partial t}\left\{\left(1-\frac{\rho_{0} c_{0}^{2}}{\rho c^{2}}\right) \frac{D p}{D t}-\frac{\left(p-p_{0}\right)}{\rho} \frac{D \rho}{D t}\right\} \\
& +\frac{\partial^{2}}{\partial x_{i} \partial t}\left(u_{i} \rho_{e}\right) .
\end{aligned}
$$

The equation above is Dowling's [1992] combusting form of Lighthill's acoustic analogy.

It is also possible to include the volumetric heat released by combustion in equation 31 by reformulating Dowling's equation. From the chain rule we have

$$
\left(\frac{\partial h}{\partial Y_{n}}\right)_{T, p, Y_{m}}=\left(\frac{\partial h}{\partial Y_{n}}\right)_{\rho, p, Y_{m}}+\left(\frac{\partial h}{\partial \rho}\right)_{p, Y_{n}}\left(\frac{\partial \rho}{\partial Y_{n}}\right)_{T, p, Y_{m}} .
$$


A rearrangement of the expression above yields

$$
\begin{aligned}
\sum_{n=1}^{N}\left(\frac{\partial h}{\partial Y_{n}}\right)_{\rho, p, Y_{m}} \rho \frac{D Y_{n}}{D t} & =\sum_{n=1}^{N}\left(\frac{\partial h}{\partial Y_{n}}\right)_{T, p, Y_{m}} \rho \frac{D Y_{n}}{D t} \\
& -\rho\left(\frac{\partial h}{\partial \rho}\right)_{p, Y_{n}}\left[\sum_{n=1}^{N}\left(\frac{\partial \rho}{\partial Y_{n}}\right)_{T, p, Y_{m}} \frac{D Y_{n}}{D t}\right] .
\end{aligned}
$$

When diffusion of species is neglected then $\rho \frac{D Y_{n}}{D t}=\omega_{n}$. Therefore, the first term on the RHS of equation 33 is equal to $-\dot{\omega}_{T}$, where $\dot{\omega}_{T}$ is the volumetric heat release $\left(W / m^{3}\right)$. The term in the square brackets in equation 33 is the change in density at constant $p$ and $T$ due to changes in species concentration. For an ideal gas $p=R T \rho / W_{0}$, where $R$ is the universal gas constant and $W_{0}$ is the average molecular weight. If the average molecular weight is constant,

$$
\sum_{n=1}^{N}\left(\frac{\partial \rho}{\partial Y_{n}}\right)_{T, p, Y_{m}} \frac{D Y_{n}}{D t}=0
$$

and equation 33 reduces to

$$
-\sum_{n=1}^{N}\left(\frac{\partial h}{\partial Y_{n}}\right)_{\rho, p, Y_{m}} \rho \frac{D Y_{n}}{D t}=\dot{\omega}_{T} .
$$

Hence, Dowling's reformulation (i.e. equation 31) of Lighthill's inhomogeneous wave equation can be written as follows.

$$
\begin{aligned}
\frac{1}{c_{0}^{2}} \frac{\partial^{2} p}{\partial t^{2}}-\nabla^{2} p & =\frac{\partial}{\partial t}\left(\frac{\alpha \rho_{0}}{c_{p} \rho}\left\{\dot{\omega}_{T}-\nabla \cdot \mathbf{q}+\frac{\partial u_{i}}{\partial x_{j}} \tau_{i j}\right\}\right) \\
& +\frac{\partial^{2}}{\partial x_{i} \partial x_{j}}\left(\rho u_{i} u_{j}-\tau_{i j}\right) \\
& +\frac{1}{c_{0}^{2}} \frac{\partial}{\partial t}\left\{\left(1-\frac{\rho_{0} c_{0}^{2}}{\rho c^{2}}\right) \frac{D p}{D t}-\frac{\left(p-p_{0}\right)}{\rho} \frac{D \rho}{D t}\right\} \\
& +\frac{\partial^{2}}{\partial x_{i} \partial t}\left(u_{i} \rho_{e}\right) .
\end{aligned}
$$


Note that, the volumetric heat release term $\dot{\omega}_{T}$ in equation 36 is the sum of the enthalpy of species $n$ multiplied by the reaction rate of that species,

$$
\dot{\omega}_{T}=-\sum_{n=1}^{N} h_{n} \dot{\omega}_{n}=-\sum_{n=1}^{N}\left(h_{s, n}+\triangle h_{f, n}^{0}\right) \dot{\omega}_{n}
$$

where $h_{s, n}$ is the sensible enthalpy of species $n$ and $\triangle h_{f, n}^{0}$ is the formation enthalpy of that species. If single-step chemistry is selected for combustion analysis, the reaction process can be described by

$$
\sum_{n=1}^{N} \nu_{n 1}^{\prime} M_{n} \rightarrow \sum_{n=1}^{N} \nu_{n 1}^{\prime} M_{n}
$$

where $M_{n}$ is a symbol for species $n, \nu_{n 1}^{\prime}$ and $\nu_{n 1}^{\prime}$ are the molar stoichiometric coefficients of species $n$. The reaction rates $\dot{\omega}_{n}$ for each species are linked to the progress rate $Q_{1}$ at which the single reaction proceeds:

$$
\dot{\omega}_{n}=W_{n} \nu_{n 1} Q_{1}
$$

where $\nu_{n 1}=\nu_{n 1}^{\prime}-\nu_{n 1}^{\prime}$ and $W_{n}$ is molecular weight of species $n$. By inserting equation 39 in equation 37 we have

$$
\dot{\omega}_{T}=-\sum_{n=1}^{N} h_{n} W_{n} \nu_{n 1} Q_{1}=-Q_{1} \sum_{n=1}^{N} h_{n} W_{n} \nu_{k 1}=\left|\nu_{F 1}\right| Q^{m} Q_{1}
$$

where $Q^{m}$ is the molar heat of reaction given by

$$
Q^{m}=-\sum_{n=1}^{N} h_{n} W_{n} \frac{\nu_{n 1}}{\left|\nu_{F 1}\right|}=-\sum_{n=1}^{N} h_{n}^{m} \frac{\nu_{n 1}}{\left|\nu_{F 1}\right|}=\sum_{n=1}^{N} h_{n}^{m} \frac{\nu_{n 1}}{\nu_{F 1}} .
$$

Note that $\nu_{F 1}=\nu_{F 1}^{\prime}-\nu_{F 1}^{\prime}$ is negative and $Q^{m}$ measures the heat released by the complete combustion of 1 mole of fuel. It is often useful to work with the heat of reaction per unit mass of fuel. To do this, the quantity $Q$ is defined which 
stands for the heat released by the complete combustion of $1 \mathrm{~kg}$ of fuel:

$$
\dot{Q}=-\frac{Q^{m}}{W_{F}}=-\sum_{n=1}^{N} h_{n} \frac{W_{n} \nu_{n 1}}{W_{F} \nu_{F 1}} .
$$

By inserting equation 42 into equation 40 and considering the reaction rate of fuel species as $\dot{\omega}_{F}=W_{F} \nu_{F 1} Q_{1}$, the following expression for the volumetric heat release by combustion is achieved when a single-step, irreversible reaction mechanism is used.

$$
\dot{\omega}_{T}=\dot{Q} \dot{\omega}_{F}
$$

Note that the heat released by the complete combustion of $1 \mathrm{~kg}$ of fuel $Q$ is negative, denoting an exothermic reaction. If all heat capacities of species are equal, i.e. $C_{p, k}=C_{p}$, equation 42 for the heat released by the complete combustion of $1 \mathrm{~kg}$ of fuel will hold the following form based on the formation enthalpy $\triangle h_{f, n}^{0}$ of species $n$ :

$$
\dot{Q}=-\sum_{n=1}^{N} \triangle h_{f, n}^{0} \frac{W_{n} \nu_{n 1}}{W_{F} \nu_{F 1}} .
$$

